Ring Resonator-Based Broadband Photonic Beam Former for Phased Array Antennas

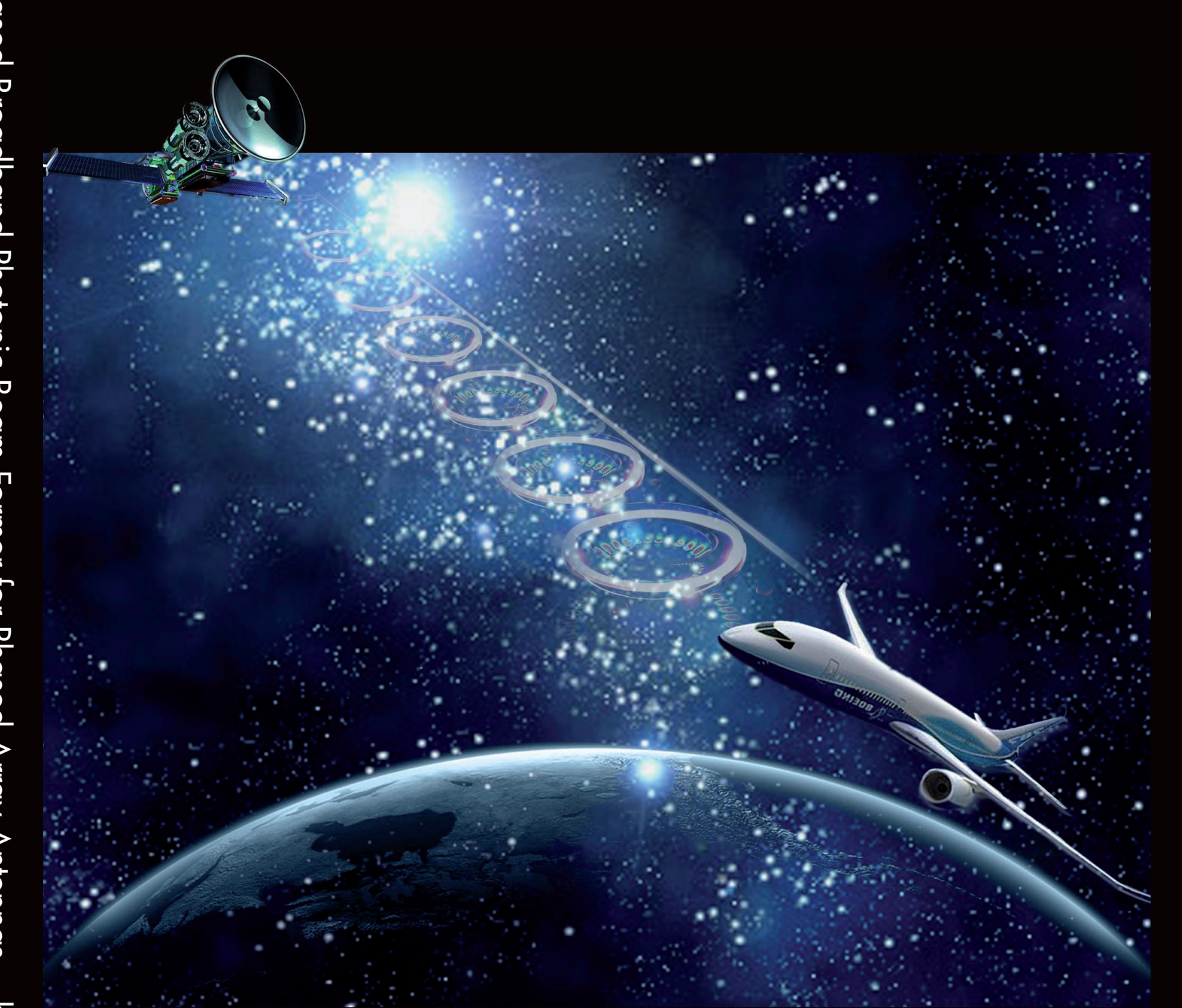

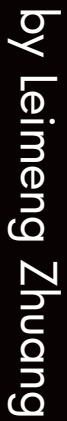

\section{Invitation}

for the participation of the public defense of my doctoral thesis, entiteld:

Ring Resonator-Based Broadband Photonic Beam Former for Phased Array Antennas

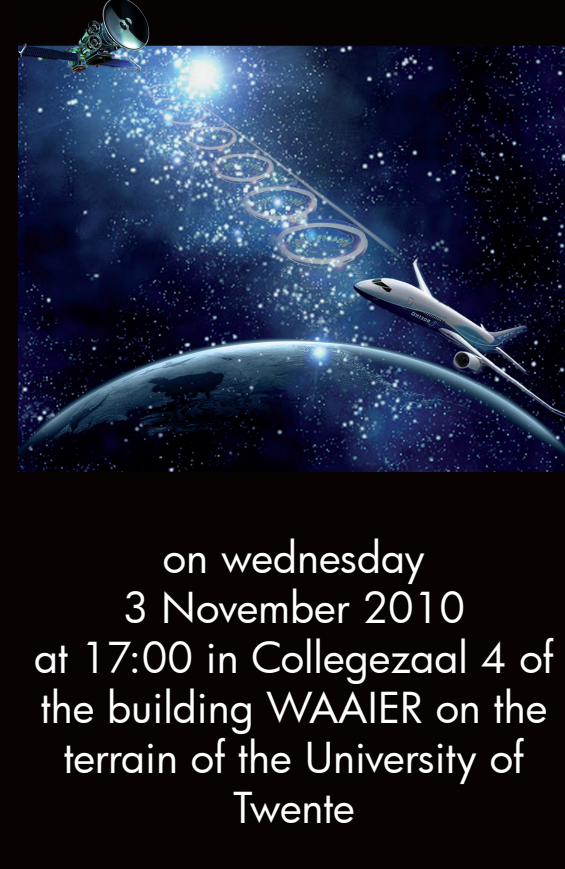

Before the defense I will give a short introduction of the content of my thesis at 16:45
Leimeng Zhuang 0645248488 I.zhuang@ewi.utwente.nl 


\title{
Ring Resonator-Based Broadband Photonic Beam Former for Phased Array Antennas
}

\author{
Leimeng Zhuang
}


The research presented in this thesis was carried out at the Telecommunication Engineering group, Faculty of Electrical Engineering, Mathematics and Computer Science, University of Twente P.O.Box 217, 7500 AE Enschede, The Netherlands.

The work described in this thesis is supported by the Dutch Ministry of Economic Affairs under the SMart Antenna system for Radio Transceivers (SMART) project and the Broadband Photonic Beamformer (BPB) project under SenterNovem project numbers IS053030 and IS052081, respectively.

Copyright $(2010$ by Leimeng Zhuang

ISBN: 978-90-365-3078-1 


\title{
RING RESONATOR-BASED BROADBAND PHOTONIC BEAM FORMER FOR PHASED ARRAY ANTENNAS
}

\section{DISSERTATION}

\author{
to obtain \\ the degree of doctor at the University of Twente, \\ on the authority of the rector magnificus, \\ Prof. dr. H. Brinksma, \\ on account of the decision of the graduation committee, \\ to be publicly defended \\ on Wednesday 3 November 2010 at 16:45
}

by

Leimeng Zhuang

born on 15 October 1980

in Beijing, China 
This doctoral thesis has been approved by:

The promoter: prof. dr. ir. W. van Etten

The assistant promoter: dr. ir. C.G.H. Roeloffzen 


\section{Summary}

This thesis presents the principles and a demonstration of optical ring resonator (ORR)-based broadband photonic beam former for phased array antennas. In Chapter 1 an introduction of RF photonics is given. The SMART and BPB projects are summarized, which are aimed for the development of ORR-based broadband photonic beam former for phased array antennas. In the SMART project the antenna is used for the aeronautic communication of Ku-band signals; in the BPB project the antenna is part of a radio telescope which is used for astronomical research. In Chapter 1 the principles of phased array antennas and optical beam forming are also explained. In Chapter 2 the principles of using a single and cascaded ORRs as delay elements are described. The ORRs are studied by means of their mathematical models. Moreover, a formula is derived to calculate the necessary number of ORRs for a desired delay response, which is useful for the design of the ORR-based optical beam forming networks (OBFNs). Basically an OBFN consists of delay elements (DEs) and combining circuitry. In this thesis only the single-wavelength schemes for the OBFN are discussed where coherent optical combining is required. In Chapter 3 different OBFN architectures are compared with respect to the system complexity and feasibility. It appears that the asymmetrical binary-tree structured OBFN results in the lowest system complexity compared to its counterparts, and it can be used for both linear (1-dimensional) and planar (2-dimentional) PAAs. In Chapter 4 different schemes for $\mathrm{E} / \mathrm{O}$ and $\mathrm{O} / \mathrm{E}$ conversions of the photonic beam former are compared with respect to optical bandwidth efficiency and system complexity. Finally, a practical architecture for the entire beam former system is found, which includes frequency down-conversion (FDC), optical sideband filter (OSBF)-based single sideband-suppressed carrier (SSB-SC) modulation, optical carrier reinsertion and balanced coherent optical detection. The FDC significantly relaxes the bandwidth requirements on the optical modulators; the SSB-SC modulation relaxes the bandwidth requirements on the OBFN and detectors; the use of coherent optical detection enhances the signal dynamic range of the beam former. Based on this practical architecture, the functional modules of beam former systems for the FLY and SKY project are designed. Then in Chapter 5 the realization processes of the FLY and SKY beam former chips are described. The beam former chip includes OBFN, OSBF, and the optical carrier reinsertion coupler. Low fabrication cost and simple implementation are achieved, since the waveguide fabrication is based on the LPCVD process and requires only one photolithography step. Thermo-optical tuning mechanism is chosen, which provides sufficient tuning speed, introduces negligible additional loss in the waveguide, and brings no implementation difficulty. Chromium and gold are used for the heaters and leads, respectively. Active fiber 
alignment and butt-coupling techniques are used for the coupling between the chip and fibers. Moreover, glue is used for fixation, which is characterized by good stability and negligible additional loss. In Chapter 6 the control system for the beam former chip is described. An algorithm for calculating the voltages supplied to the heaters is introduced, which includes the compensation for the thermal crosstalk. The sensitivity of the beam steering is determined by the accuracy of the voltage output of the heater controller. The heat generated by the heaters may change the chip temperature and consequently causes optical parameter fluctuations. To solve this problem, a dedicated temperature stabilization setup is used, which consists of a heat conducting copper base, Peltier elements and a heat sink. Then, when the maximum pump power of the Peltier elements is larger than the maximum power dissipation of the heaters on the chip, the magnitude of the fluctuation can be well controlled by properly choosing the heat capacity of the heat conducting base. In Chapter 7 the characterizations of the FLY and SKY beam former chips are presented. The beam former chips are designed for the wavelength of $1550 \mathrm{~nm}$. The minimum insertion loss of the chips is approximately $12 \mathrm{~dB}$. The components on both FLY and SKY beam former chips prove to function correctly. The realized ORRs are able to provide continuous tunable delays. A set of linearly increasing delays on different channels of a test OBFN are achieved, which matches the desired OBFN functionality. For a test channel using 7 ORRs, a delay value of $1.2 \mathrm{~ns}(36 \mathrm{~cm}$ physical distance in air) is achieved for a bandwidth of $2.5 \mathrm{GHz}$ and the maximum delay ripple of $0.1 \mathrm{~ns}$. A test OSBF was tuned to its optimized response, which is also in perfect match with theory. Three delay settings with $0 \mathrm{ps}, 20 \mathrm{ps}$ and $40 \mathrm{ps}$ delay steps between the neighboring channels are created on the FLY beam former chip, which corresponds to the theoretical beam angles of $-30,0$, and 30 degree, respectively. Likewise, two delay settings with 0 ps and 110 ps delay steps between the neighboring channels are created on the SKY beam former chip, which corresponds to the theoretical beam angles of 0 and 10 degree, respectively. Further, the FLY beam former chip was implemented in a complete beam former system (the beam former demonstrator built at $\mathrm{NLR}^{1}$ ). With this setup, some RF-to-RF measurements were performed, which demonstrate the functionalities of the beam former chip: optical sideband suppression, RF-to-RF delay generation, and coherent combining. Finally, an experiment of antenna beam steering was performed on the FLY beam former demonstrator using the three delay settings mentioned above, and three optically controlled beam angles of $-27,0$, and 27 degree are successfully obtained. The presented measurements and demonstrations verify the device functionalities, and prove the concept of PAA control using ORR-based beam former system.

\footnotetext{
${ }^{1}$ National Aerospace Laboratory (NLR). Address: Voorsterweg 31, 8316 PR Marknesse, P.O.Box 153, 8300 AD, Emmeloord, the Netherlands. Telephone: (+31) 527248444.
} 


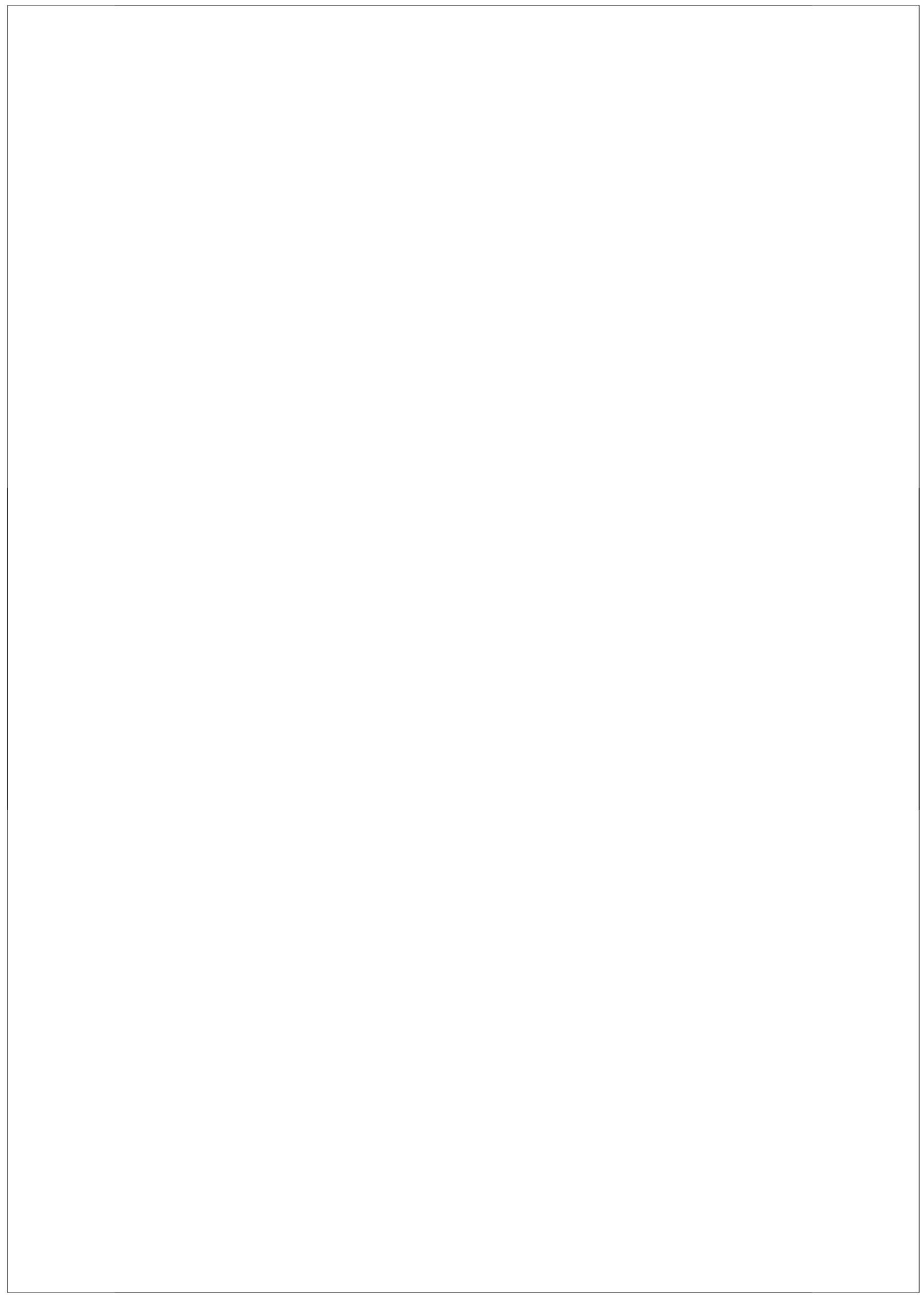




\section{Contents}

Abbreviations I

Symbols II

Chapter 1: Introduction 1

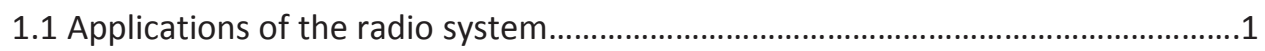

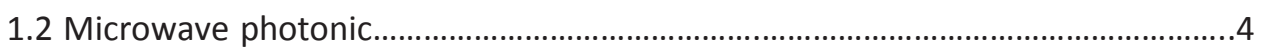

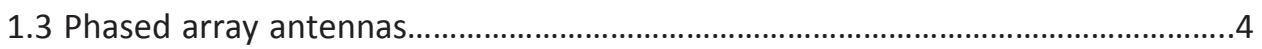

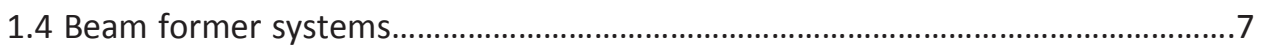

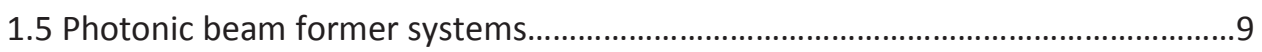

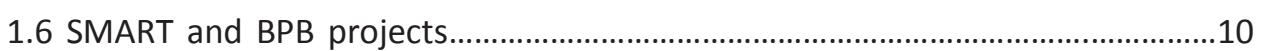

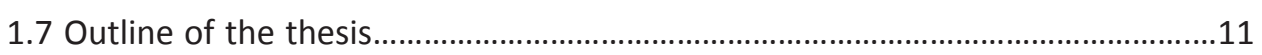

Chapter 2: Optical ring resonator-based delay lines 13

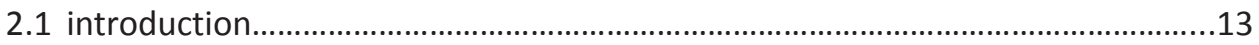

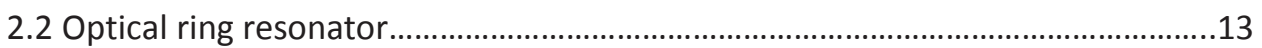

2.3 Digital filter concepts for optical filters.................................................................13

2.3.1 The transfer function of the waveguide feedback path.........................14

2.3.2 The transfer matrix of the $2 \times 2$ optical coupler.....................................16

2.3.3 The transfer function of the ORR.........................................................19

2.3.4 Time domain analysis..........................................................................20

2.3.5 Optical all-pass filter.............................................................................21

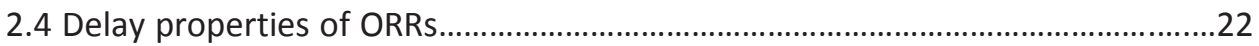

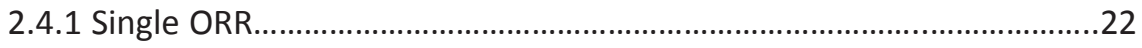

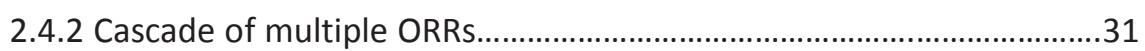

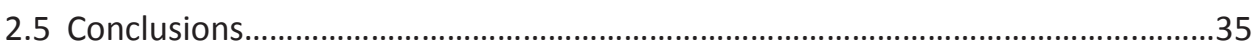

\section{Chapter 3: Ring resonator-based optical}

beam forming networks 37

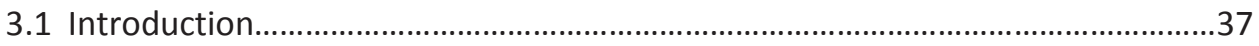

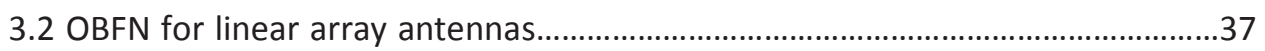

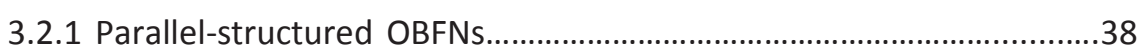

3.2.2 Binary-tree-structured OBFNs...................................................42 
3.3 OBFN for rectangular $M \times N$ planer array antennas............................................46

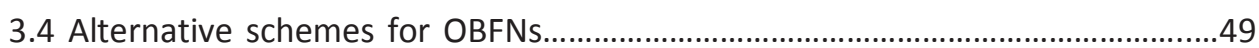

3.5 Optical combiner and phase shifter....................................................................57

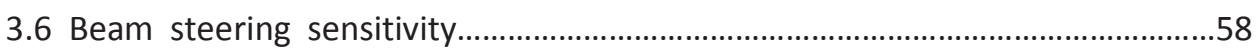

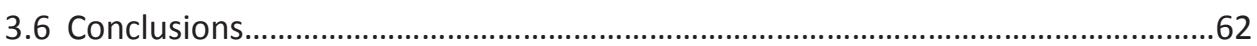

\section{Chapter 4: Advanced signal processing techniques} for beam former systems $\quad 65$

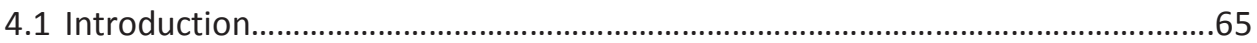

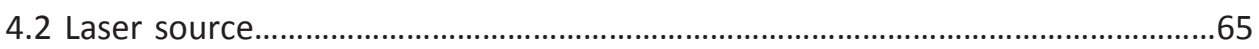

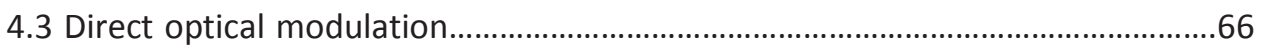

4.4 Optical DSB modulation and direct optical detection..........................................67

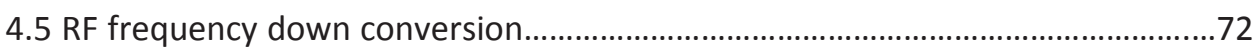

4.6 Optical SSB-SC modulation and balanced coherent optical detection..................73

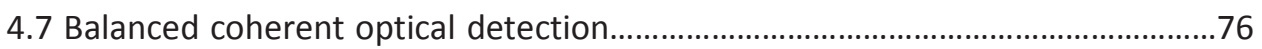

4.8 Combination of FDC and optical SSB-SC modulation............................................77

4.9 Implementation of optical SSB-SC modulation....................................................78

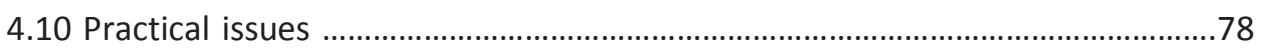

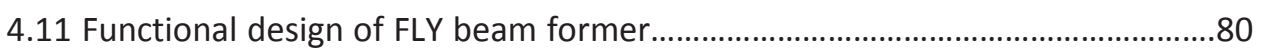

4.12 Functional design of SKY beam former...............................................................85

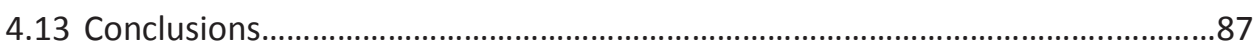

Chapter 5: Beam former chip realization $\quad 91$

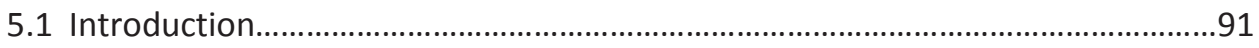

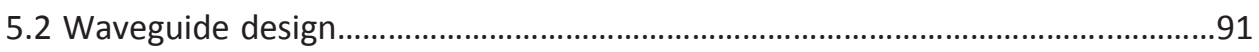

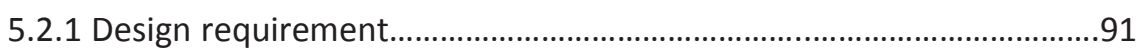

5.2.2 Waveguide characteristics.....................................................................92

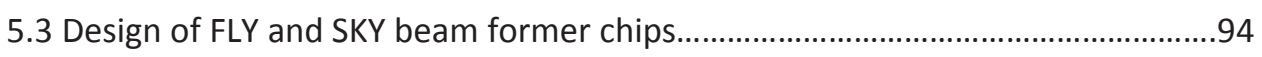

5.3.1 Design of optical phase shifter...........................................................94

5.3.2 Design of tunable optical power coupler..................................................96

5.5.3 Design of ORRs.................................................................................96

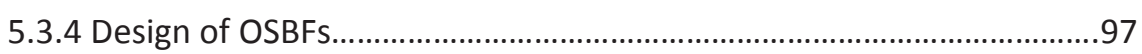

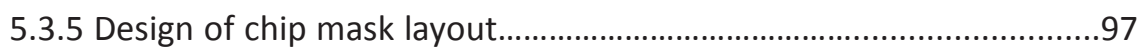

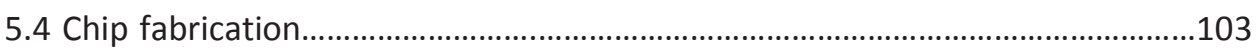

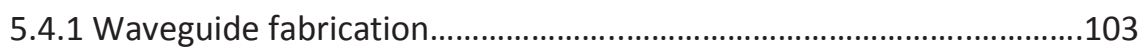

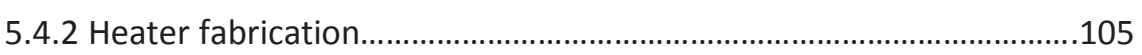

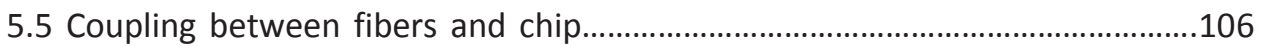


6.1 Introduction

6.2 Heater controller

6.2.1 Design requirement

6.2.2 Configuration of the designed heater controller.

6.3 Heater voltage calculation.

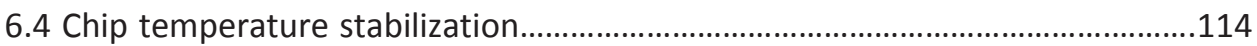

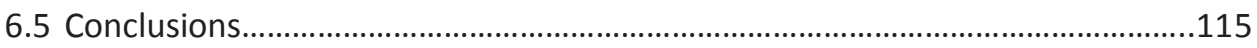

Chapter 7: Chip characterization $\quad 117$

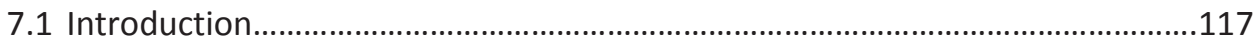

7.2 Characterization of beam former chip...........................................................117

7.2.1 Measurement setup..........................................................................117

7.2.2 Measurement steps.........................................................................120

7.2.3 Measurement of the insertion loss of the chip.................................121

7.2.4 Characterization of the MZI coupler.................................................122

7.2.5 Characterization of the ORR-based OBFN...........................................123

7.2.6 Characterization of the OSBF.........................................................128

7.2.7 Characterization of the FLY beam former chip.................................129

7.2.8 Characterization of the SKY beam former chip...................................133

7.3 RF-to-RF measurements on the beam former system......................................135

7.3.1 Sideband suppression.........................................................................136

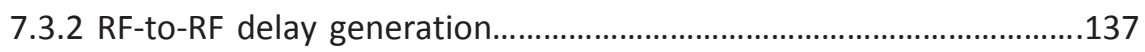

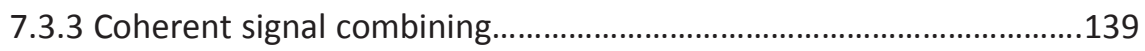

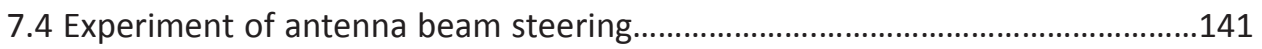

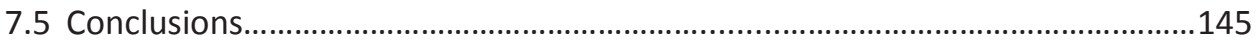

Chapter 8: Conclusions and future directions 149

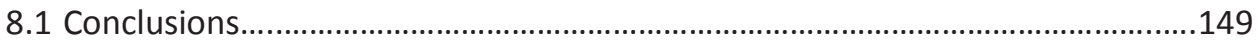

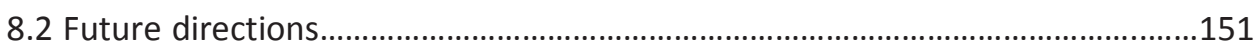

$\begin{array}{ll}\text { Appendix } & 155\end{array}$

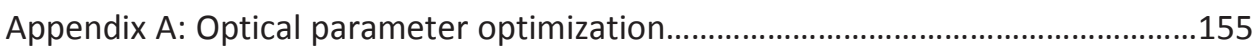


Appendix B: Heater crosstalk compensation..

Publications

Acknowledgement

165 


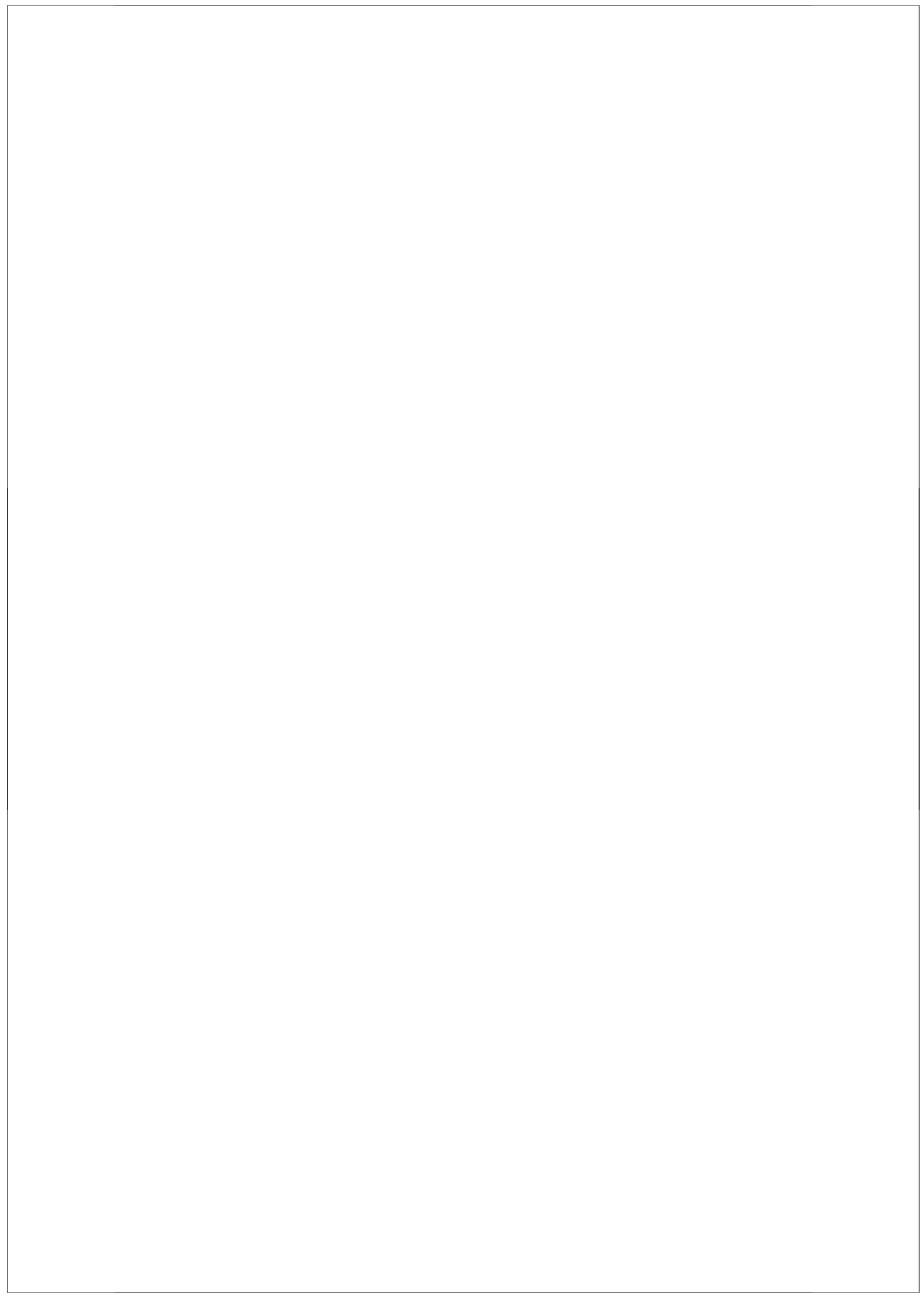




\section{Abbreviations}

\begin{tabular}{|c|c|}
\hline $\mathrm{AE}$ & Antenna element \\
\hline $\mathrm{BFN}$ & Beam forming network \\
\hline $\mathrm{BPB}$ & Broadband Photonic Beamformer \\
\hline CMOS & Complementary metal-oxide-semiconductor \\
\hline $\mathrm{CW}$ & Continuous wave \\
\hline $\mathrm{DC}$ & Directional coupler \\
\hline DD & Direct detection \\
\hline $\mathrm{DE}$ & Delay element \\
\hline DSB & Double-sideband \\
\hline EAM & Electro-absorption modulator \\
\hline $\mathrm{E} / \mathrm{O}$ & Electro-optical \\
\hline FAUs & Fiber array units \\
\hline FSR & Free spectral range \\
\hline FDC & Frequency down conversion \\
\hline IIR & Infinite impulse response \\
\hline IF & Intermediate frequency \\
\hline IM & Intensity modulation \\
\hline IMD & Intermodulation distortion \\
\hline LNA & Low-noise amplifier \\
\hline LO & Local oscillator \\
\hline LPCVD & Low-pressure chemical vapor deposition \\
\hline MFD & Mode field diameter \\
\hline MZI & Mach-Zehnder interferometer \\
\hline MZM & Mach-Zehnder modulators \\
\hline OBFN & Optical beam forming network \\
\hline OBI & Optical beat interference \\
\hline OCDMA & Optical code-division multiple access \\
\hline $\mathrm{O} / \mathrm{E}$ & Opto-electrical \\
\hline OPS & Optical phase shifter \\
\hline ORR & Optical ring resonator \\
\hline OSBF & Optical sideband filter \\
\hline PAA & Phased array antennas \\
\hline PDL & Polarization dependent loss \\
\hline PECVD & Plasma enhanced chemical vapor deposition \\
\hline PMF & Polarization maintaining fiber \\
\hline SEM & Scanning electron microscope \\
\hline SSB-SC & Single sideband-suppressed carrier \\
\hline $\mathrm{RF}$ & Radio frequency \\
\hline SMART & Smart antenna system for radio transceivers \\
\hline TE & Transverse electric \\
\hline TEOS & Tetraethyl orthosilicate \\
\hline TM & Transverse magnetic \\
\hline VCSEL & Vertical-Cavity Surface-Emitting Laser \\
\hline
\end{tabular}




\section{Symbols}

\begin{tabular}{cl}
$a(t)$ & Time-dependent amplitude \\
$\alpha$ & Attenuation coefficient \\
$c$ & Bar-port transmission coefficient of the $2 \times 2$ coupler \\
$c_{o}$ & Speed of light in vacuum \\
$d$ & Interelement distance of the phased array antenna \\
$E_{a}$ & Complex amplitude of the bandpass signal \\
$E_{L}$ & Complex amplitude of laser output \\
$E^{i}$ & Complex amplitudes of the electric fields at input of the waveguide \\
$E^{o}$ & Complex amplitudes of the electric fields at output of the waveguide \\
$\Delta f_{\mathrm{BW}}$ & ORR delay bandwidth \\
$f_{\mathrm{OF}}$ & Optical carrier frequency \\
$f_{\mathrm{RF}}$ & RF carrier frequency \\
$\Delta f_{S}$ & Signal bandwidth \\
$H_{L}$ & Complex transfer function \\
$|H(\Omega)|$ & Magnitude response \\
$\left.I_{\mathrm{out}} \mathrm{t}\right)$, & Output current of the photodiode \\
$K$ & Number of stages \\
$L$ & Length \\
$M T$ & Maximum time offset of the antenna element \\
$N_{\mathrm{TT}}$ & Total amount of ORRs in the OBFN \\
$n_{e}$ & Effective index of the waveguide \\
$n_{g}$ & Group index of the waveguide \\
$\Delta n_{g}$ & Deviation of the group index \\
$k_{O}$ & Zero-order wave number \\
$P$ & Power \\
$P_{L}$ & Roundtrip loss \\
$q_{n}$ & Amplitude coefficient \\
$R_{\mathrm{PD}}$ & Responsivity of the photodiode \\
$S_{n}(t)$ & Modulating signal \\
$S(V)$ & Beam steer sensitivity to heater voltage \\
$t$ & Time \\
$T$ & Roundtrip time \\
$T_{\mathrm{CO}}$ & Common total delay for all antenna elements \\
$\Delta t_{a}$ & Wavefront arrival time difference between antenna elements \\
$V$ & Voltage \\
$\zeta$ & Phase factor \\
$\kappa$ & Power coupling ratio \\
$\kappa_{\mathrm{DC}}$ & Power coupling coefficient of the DC \\
$\kappa_{\mathrm{MZI}}$ & Power coupling coefficient of the MZI \\
$\kappa_{r}$ & Power coupling coefficient of the ORR in the OSBF \\
$\lambda$ & Wavelength \\
$\tau_{d}$ & Normalized group delay \\
& Delay difference between the MZI arms \\
\hline & \\
\hline &
\end{tabular}




$\begin{array}{cl}\tau_{g} & \text { Group delay } \\ \bar{\tau} & \text { Average delay value over the delay band } \\ \Delta \tau & \text { Delay ripple normalized to the roundtrip delay of the ORR } \\ \Delta \tau_{d} & \text { Delay difference between two AEs provided by the BFN } \\ \theta & \text { Beam angle of the PAA } \\ \Delta \theta & \text { Beam scanning range of the PAA } \\ \phi & \text { Additional phase shift of the ORR } \\ \phi_{d} & \text { Additional phase shift of the MZI in the OSBF } \\ \phi_{r} & \text { Additional phase shift of the ORR in the OSBF } \\ \phi\left(f_{\mathrm{OF}}\right) & \text { Phase of the optical carrier } \\ \varphi(t) & \text { Time-dependent phase offset } \\ \Delta \varphi & \text { Phase difference between two AEs provided by the BFN } \\ \Psi(\Omega) & \text { Phase response } \\ \Delta \Omega_{\mathrm{BW}} & \text { Delay bandwidth normalized to the FSR of the ORR } \\ \Omega & \text { Angular frequency normalized to the FSR of the ORR } \\ \omega & \text { Angular frequency }\end{array}$




\section{Chapter 1}

\section{Introduction}

\subsection{Applications of radio systems}

In the last century radio systems became an essential part of everyone's life. Radio systems are used to pass on signals by means of the transmission of electromagnetic waves with frequencies below those of visible light. In the modern world some of the most popular applications of radio systems can be seen in the well-known areas such as audio/video broadcast, telephony, navigation, radar, satellite, astronomy, heating, and radio control.

In the recent years more ideas of broadband radio applications have been brought forth and attract great attention. One of them is motivated by the ever-increasing demand for fast information exchange, especially for the availability of fast internet. People wish to have internet access and multimedia services no matter where they are. For example, some people wish to surf the internet, participate in a teleconference, or watch live TV during a flight travel, especially when it is a long intercontinental flight travel. However, when the plane is flying over the sea, there is no direct radio link between the airplane and ground available. In this case the radio link can only be established via a satellite. Then, this demand concerns the development of aeronautic communication for personal and multimedia services via satellite.

This development is still at an early stage. However, it is seen as the main growth driver for mobile satellite services in the coming decade. It is reported in the International Telecommunication Union News of November 2009 that Euroconsult (international analysts specialized in satellite communication) expects the market for aeronautic communication via satellite to significantly grow reaching wholesale revenues of more than USD 270 million in 2018 [1]. In order to provide the demanded aeronautic communication services to their passengers, the air carrier companies need an antenna-based RF system for the link between ground station, satellite, and airplane, which is shown in Figure 1.1.

Besides the aeronautic communication, another interesting broadband radio application is the radio telescope used for radio astronomy. Radio astronomy is a subfield of astronomy which investigates the radiation from celestial objects at radio 
frequencies. For this purpose radio telescopes must be developed for receiving the radio waves emitted from those bodies.

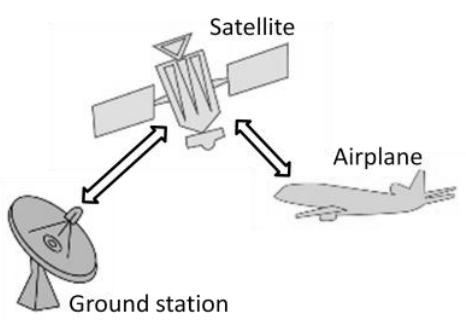

Figure 1.1: Schematic drawing of the aeronautic communication system

Radio astronomy can be conducted with a single dish radio telescope. However, in most cases, the angular resolution of a single radio telescope is difficult to satisfy the requirement of the study [2]. To achieve higher resolution, the techniques of radio interferometry and aperture synthesis can be used, which means an array of multiple linked radio telescopes will be used instead of a single one [2]. The realization of such a radio system is a big concern nowadays in the research field of radio communication. A picture of an interferometric array formed by many dish antennabased radio telescopes is shown in Figure 1.2.

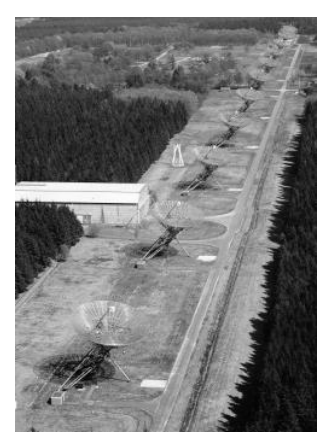

Figure 1.2: A photo of Westerbork Synthesis Radio Telescope (WSRT) at Astron ${ }^{2}$ (picture source: Google)

In the two radio applications described above: aeronautic communication via satellite and radio telescope, both of the systems are featured by the radio link with low signal power density. Therefore, high-gain and direction-sensitive antennas are required for the signal reception. Ordinary omni-directional antennas are not preferable in these radio applications because of the low antenna gain [3].

\footnotetext{
${ }^{2}$ Netherlands Institute for Radio Astronomy (Astron). Address: Oude Hoogeveensedijk 4, 7991 PD, Dwingeloo, The Netherlands. Telephone: (+31) 521595100.
} 
A conventional solution for a direction sensitive antenna is a dish antenna [2], which can be steered mechanically to point its main beam to different directions. However, its tuning precision and speed are limited by the mechanical movement. In the airborne case the use of dish antennas is hampered by the drawbacks of its large weight, large size and aerodynamic drag effect when mounted on top of a plane. Besides in the case of radio astronomy the dish antenna-based telescopes are usually huge and spaced far apart from one another in a telescope array, which results in great difficulty for the maintenance of the telescopes.

Instead of using dish antennas an advantageous alternative is to use phased array antennas (PAAs) [3], [4]. A PAA consists of an array of antenna elements (AEs) as shown in Figure 1.3, and its antenna pattern is determined by the geometry of the array as well as the signal amplitude and phase relation between the AEs. By changing the amplitude and phase relation, the main beam of a PAA can be steered without any involvement of mechanical movement. The principles of this will be further explained in Section 1.4. However, to achieve this function a so-called beam former is required for the PAA. A beam former is a dedicated circuit which controls both the amplitudes and phases of the AE signals. The principles of the beam former will be further explained in Section 1.5. Conventionally a beam former is realized in the electrical domain. However, it can also be realized in the optical domain: a photonic beam former. A photonic beam former applies the principles of microwave photonics where RF signals are modified by optical devices. Compared to its electrical counterpart, the photonic beam former has the advantages, such as compactness, light weight, low loss, frequency independence, large instantaneous bandwidth, and inherent immunity to electromagnetic interference. Apparently PAA comprising photonic beam former can be a preferable solution for the signal reception in the radio applications mentioned above.

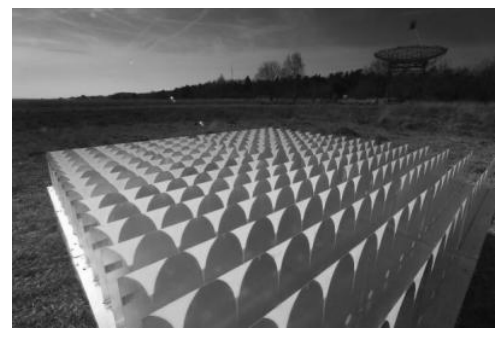

Figure 1.3: An artistic impression of the out-door phased array system Thousand Element Array (THEA) at Astron ${ }^{3}$, which consists of 256 broadband receiving antenna elements (picture source: www.astron.nl).

\footnotetext{
${ }^{3}$ Netherlands Institute for Radio Astronomy (Astron). Address: Oude Hoogeveensedijk 4, 7991 PD, Dwingeloo, The Netherlands. Telephone: (+31) 521595100.
} 


\subsection{Microwave photonics}

Microwave photonics can be defined as the field where RF signals are modified in the optical domain [5]-[7]. Its initial rationale was to use the advantages of photonic technologies to process RF signals that are very complex or even impossible to process directly in the RF domain. The significant advantages of the microwave photonic links over its electrical counterpart are reduced size, weight, and cost; low and constant attenuation over large frequency range; immunity to electromagnetic interference; low dispersion; and high data transfer capacity.

The basic components of a microwave photonic link are devices that offer electricalto-optical (E/O) signal conversion, photonic processing, and optical-to-electrical $(\mathrm{O} / \mathrm{E})$ signal conversion. Efficient $\mathrm{E} / \mathrm{O}$ conversion and $\mathrm{O} / \mathrm{E}$ conversion is imperative for photonic solutions to be competitive with conventional RF electronics. In the past, it has been the $\mathrm{E} / \mathrm{O}$ and $\mathrm{O} / \mathrm{E}$ conversion losses that have significantly impaired RF link performance. Recent progress in laser, modulator and detector component technologies are beginning to remove this impairment and create new photonic solutions for RF signal processing. As high power photodiodes with extended frequency responses are developed, RF power delivery at frequencies well beyond $10 \mathrm{GHz}$ becomes feasible [8]. Recent advancements in low noise high power laser sources, wideband lithium niobate optical modulators, and high power ultra-fast optical detectors have resulted in photonic links with impressive performance.

During the past three decades, microwave photonics has succeeded in developing and demonstrating a wide range of devices, technologies and applications, some of which have shown their commercial importance, such as optical access network and high-bit-rate data transmission [9], [10]. The photonic beam former for PAA's is also an interesting application of microwave photonics, which facilitates broadband signal reception of PAA systems. The current challenge of a microwave photonic system is to further increase its operational bandwidth, conversion efficiency and dynamic range; and in the mean time to reduce the system cost. The success in these improvements will bring forth more microwave photonics applications in the future.

\subsection{Phased array antenna}

In wave theory, a PAA is a group of AEs in which the relative phases of the respective signals feeding the AEs are varied in such a way that the effective radiation pattern of the array is intensified in a desired direction and suppressed in undesired directions [3], [4]. The intensification of the radiation pattern in the desired direction results in the main beam of the array. Figure 1.4 (a) illustrates the main beam of a radiation pattern. The direction of the main beam can be steered by 
properly changing the phase relation between AE signals, or in short the phase relation between AEs. The effect of beam steering is illustrated in Figure 1.4 (b). In the case of a receiving PAA, the antenna output is the sum of the signals (currents) of all the AEs. The signals of the AEs add constructively when they are mutually in phase and destructively when they are mutually anti-phase. According to its effective radiation pattern, a receiving PAA is able to achieve signal intensification or suppression in its output for different directions of the incident wave.

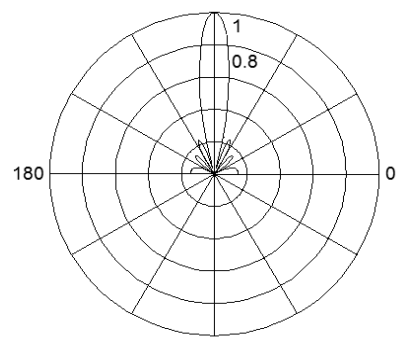

(a)

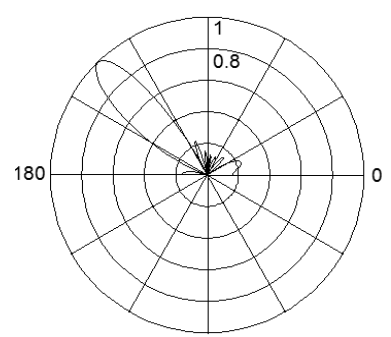

(b)

Figure 1.4: Illustration of (a) the main beam of an effective radiation pattern and (b) the beam steering effect

There are various array geometries and advanced signal processing techniques for a PAA to achieve particular effective radiation patterns [3], [4]. The simplest PAA is the one-dimensional linear array antenna. A linear array antenna is based on the geometry with multiple identical AEs equally spaced along a single line. A schematic of a 4-element linear array antenna is shown in Figure 1.5.

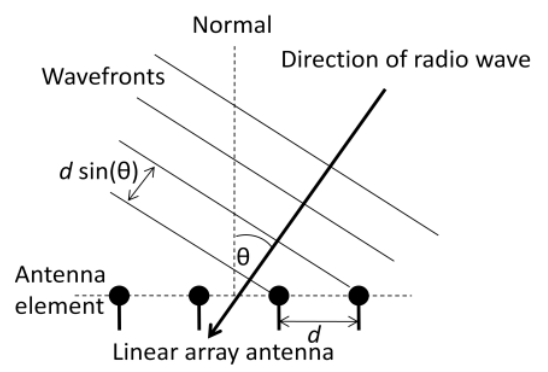

Figure 1.5: Illustration of a 4-element one-dimensional linear array antenna

Suppose it is desired to receive a plane wave coming at an angle $\theta$ with respect to the array normal. Then all the spatial points have the same phase form a wavefront which is perpendicular to propagation direction of the wave, and this wavefront will reach each $\mathrm{AE}$ at a different time instance. The arrival time difference between two neighboring AEs is given by 


$$
\Delta t_{a}(d, \theta)=d \sin (\theta) / \mathrm{c}_{0}
$$

where $d$ is the spacing between the AEs and $c_{0}$ is the speed of light in vacuum. This results in an interelement phase difference

$$
\Delta \varphi(d, \theta, f)=2 \pi f \Delta t_{a}(d, \theta)
$$

where $f$ is the frequency of the RF wave. When this interelement phase difference $\Delta \varphi(d, \theta, f)$ is removed by the additional phase compensation between two neighboring AEs $\Delta \delta=-\Delta \varphi(d, \theta, f)$, the signals from different AEs will be in phase and can be combined coherently. Then after combining the signals the intensification will be achieved for this particular receiving angle $\theta$. The signals coming from a different angle $\theta^{\prime}$ will result in $\Delta \varphi\left(d, \theta^{\prime}, f\right)$ which will not be cancelled out by $\Delta \delta$. Then due to the remaining phase difference between the AEs, suppression of the signal will occur after the signal combining of the array. Furthermore, by adjusting the value of $\Delta \delta$, the receiving direction of the array (main beam) can be steered accordingly.

For one-dimensional linear array antennas, the array geometry determines that the beam steering can only be performed in one dimension as shown in Figure 1.5. When the two-dimensional beam steering is required for some PAA application, a practical solution is to use a planar array antenna [3], [4]. A common type of planar array is the rectangular $M \times N$ planar array. A schematic of the rectangular $M \times N$ planar array antenna with interelement distance $d$ is shown in Figure 1.6. Basically, a rectangular $M \times N$ planar array can be regarded as $M$ columns of $N$-element linear arrays or $N$ rows of $M$-element linear arrays. More formal analyses of PAAs can be found in [3], [4].
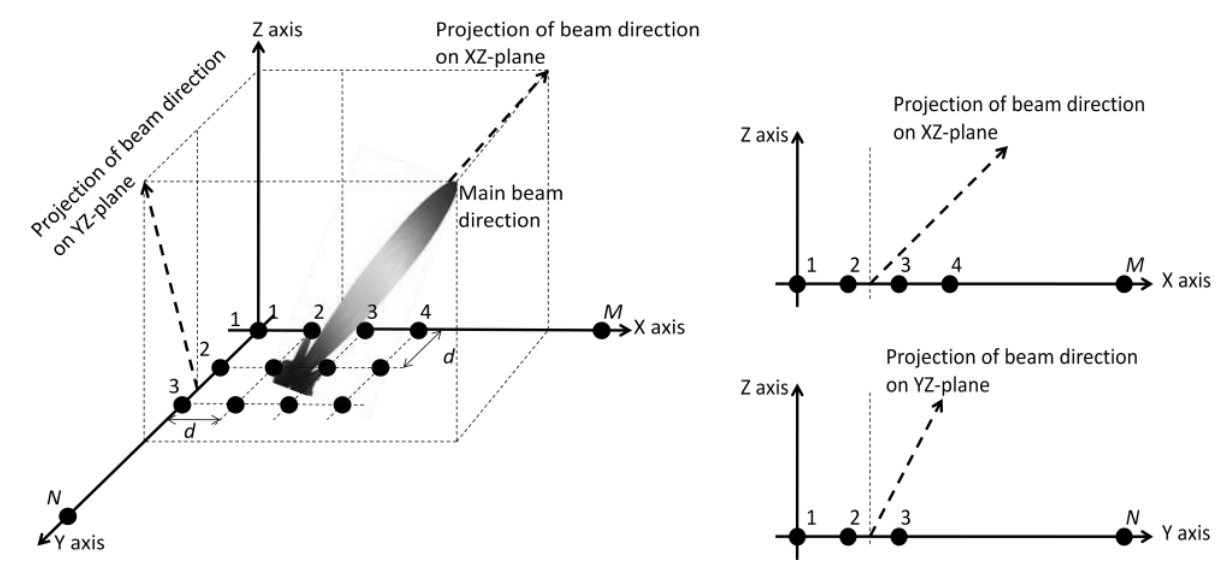

Figure 1.6: Schematic of a rectangular $M \times N$ planar array antenna 


\subsection{Beam forming systems}

As mentioned in the previous section, to steer the beam of a receiving PAA to a certain direction, the corresponding phase differences between the AEs need to be removed by intentional phase compensation. To perform that, a dedicated signal processing circuit is needed, which is called beam forming network (BFN). Basically a BFN consists of phase shifters or delays, and signal combining (or splitting in the case of transmitting antenna) circuitry [11], [12]. A schematic of the BFN for 4-element receiving PAA is shown in Figure 1.7.

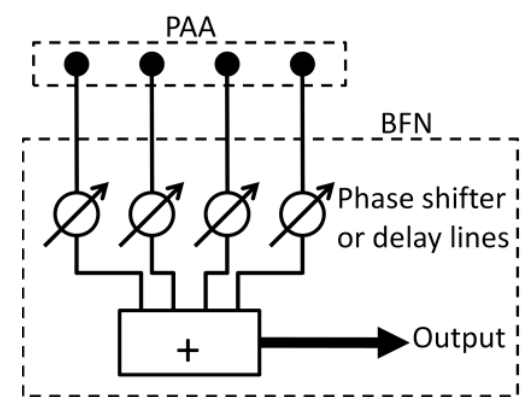

Figure 1.7: Schematic of the beam forming network for 4-element receiving PAA

The BFN is the core of the beam former system. A conventional BFN uses phase shifters to perform phase compensation. However, in this case the beam angle is frequency-dependent. Based on the linear array antenna and the BFN shown in Figure 1.5 and 1.7, respectively, the beam angle $\theta$ in radius can be given by

$$
\theta=\arcsin \left(\frac{\mathrm{c}_{0} \Delta \varphi}{2 \pi d f}\right)
$$

where $\Delta \varphi$ is the interelement phase difference provided by means of phase shifters, $\mathrm{c}_{0}$ is the speed of light in vacuum, and $d$ is the interelement distance. Further, by taking the first derivative of $\theta$ with respect to signal frequency $f$, one can obtain

$$
\frac{\mathrm{d} \theta}{\mathrm{d} f}=\left(\frac{\Delta \varphi_{\mathrm{p}} \mathrm{c}_{0}}{2 \pi d}\right) /\left(f^{2} \sqrt{1-\left(\frac{\Delta \varphi_{\mathrm{p}} \mathrm{c}_{0}}{2 \pi d f}\right)^{2}}\right)
$$

Eq. (1.4) shows that $\mathrm{d} \theta / \mathrm{d} f$ is not a constant but a function of frequency $f$. This indicates the so-called beam squinting effect. It means that for signals with a certain bandwidth, different frequency components correspond to different beam angles. An illustration of this effect is shown in Figure 1.8. A numerical example of the beam squinting effect is given in Example 1.1 where a Ku-band PAA is considered for calculation. 


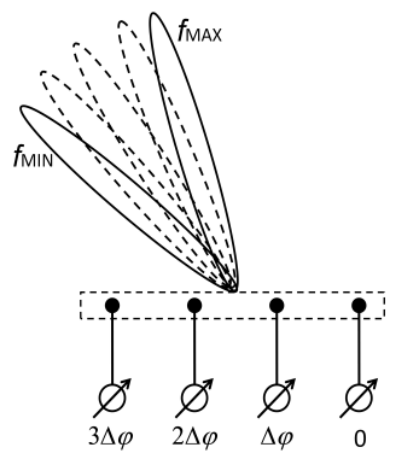

Figure 1.8: Beam squinting effect of phase shifter-based BFN for broadband signal

\section{Example 1.1}

Antenna interelement distance: $d=1.18 \mathrm{~cm}$;

Based on Eq. (1.4), the beam squinting effect $\Delta \theta$ of the phase shifter-based BFN is shown below for $1 \mathrm{GHz}$ signal bandwidth and different values of interelement phase difference $\Delta \varphi$ provided by the phase shifters.

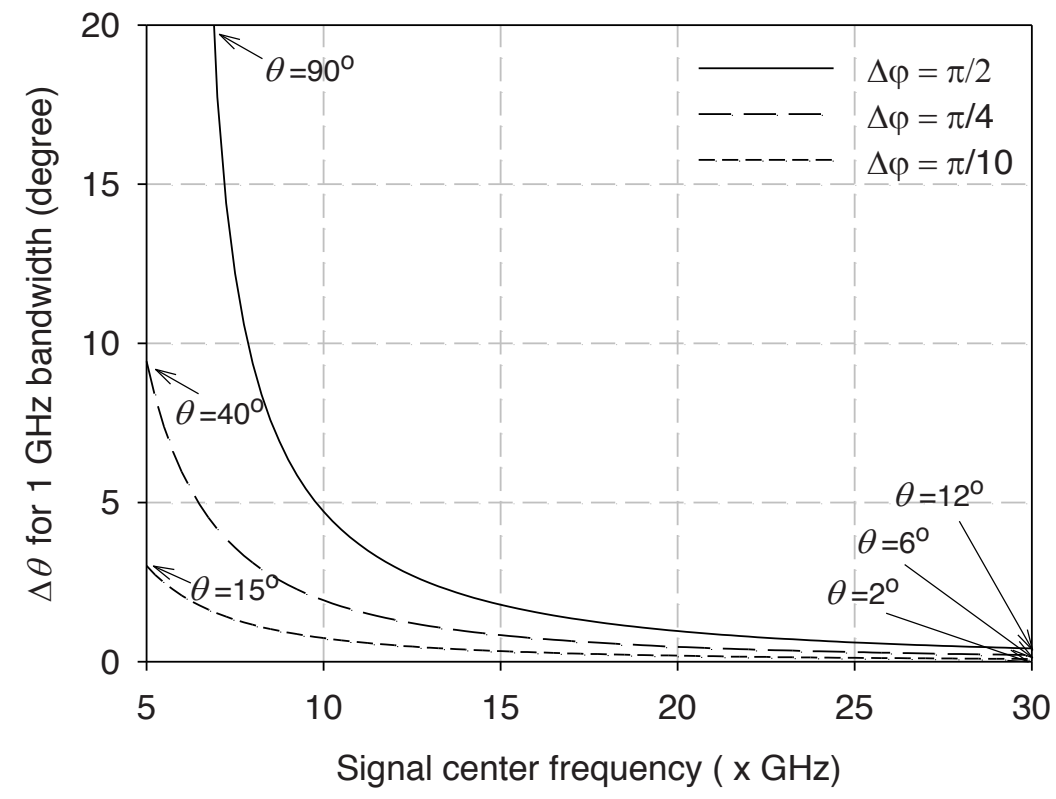

Example 1.1 shows the beam squinting effect of the BFN based on phase shifters. To avoid the beam squinting effect, delay lines should be used in the BFN instead of phase shifters. Comparatively, when a delay-based BFN is used for the linear array 
antenna, the beam angle $\theta$ and its first order derivative $B$ with respect to signal frequency $f$ can be given by

$$
\begin{gathered}
\theta=\arcsin \left(\frac{\Delta \tau_{\mathrm{d}} \mathrm{c}_{0}}{d}\right) \\
B=\frac{\mathrm{d} \theta}{\mathrm{d} f}=0
\end{gathered}
$$

where $\Delta \tau_{\mathrm{d}}$ is the interelement delay difference provided by the delay lines, $\mathrm{c}_{0}$ is the speed of light in vacuum, and $d$ is the interelement distance. In this case the beam angle $\theta$ is independent of signal frequency $f$. Therefore, for signals with a certain bandwidth, all the frequency components will follow only one beam angle.

\subsection{Photonic beam former systems}

As mentioned in the beginning of this chapter, a PAA can not only be electrically controlled but also be optically controlled, and a photonic beam former benefits from the common advantages of RF photonics. The photonic beam former follows the same beam forming principles as its electrical counterpart, but the AE signals are processed in the optical domain. In this case, the AE signals of a receiving PAA must first be converted into optical signals by means of optical modulation, and then processed and combined by means of an OBFN, and eventually the output of the OBFN is converted back into the electrical domain by means of optical detection. A block diagram of this process is shown in Figure 1.9. For transmitting antennas the signal stream is inverted, and the orders of electro-optical $(\mathrm{E} / \mathrm{O})$ and opto-electrical $(\mathrm{O} / \mathrm{E})$ conversion are reversed.

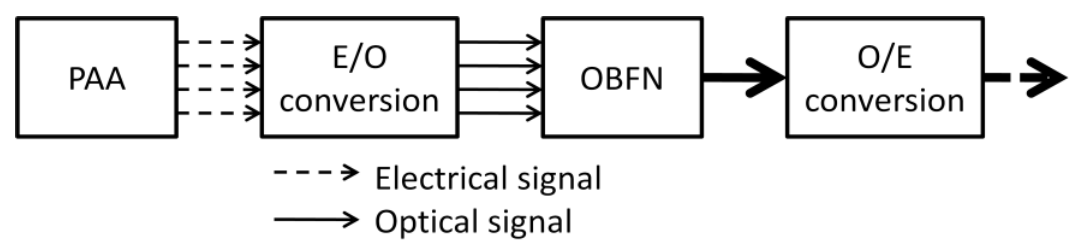

Figure 1.9: Block diagram of photonic beam former system for receiving PAA

Based on the beam forming principles explained in the previous section, optical delay lines are required in the OBFN to avoid beam squinting effect for broadband PAA applications. A well-known implementation of the OBFN is based on optical switchable delay lines, which is illustrated in Figure 1.10. However, optical switchable delay lines can only provide discrete delay values, which results in the disadvantage of the limited beam steering resolution for the PAA [12]. An alternative implementation of the OBFN that offers both time delays and continuous tunability is based on chirped fiber gratings [13], but this approach requires bulky 
optical components i.e. optical circulators and a relatively expensive tunable laser as shown in Figure 1.11. To overcome these disadvantages, the waveguide-based optical ring resonator (ORR) appears to be a good candidate for the optical delay line. ORR-based delays are able to provide continuous tunable delays. The device principles of ORRs are given in Chapter 2.

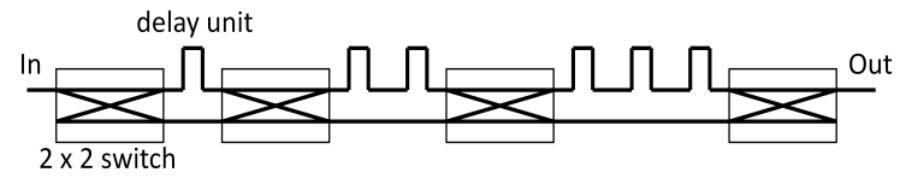

Figure 1.10: Schematic of a switchable delay line

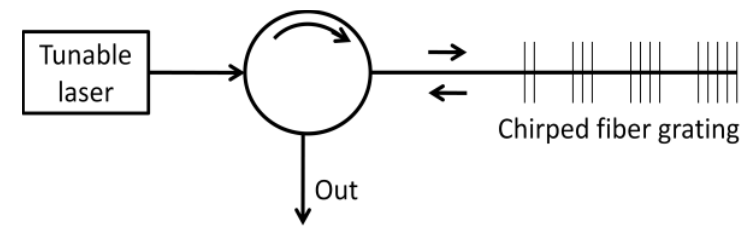

Figure 1.11: Schematic of a chirped fiber grating-based delay line

\subsection{SMART and BPB projects}

As mentioned in the previous sections, the aeronautical communication via satellite and the radio telescope are interesting subjects to apply PAA's and the photonic beam former system. However, the conventional photonic beam formers have limitations in processing bandwidth or tuning resolution or system integration due to the imperfection of the applied optical devices for signal processing [11]-[13]. To overcome these limitations, optical ring resonator (ORR)-based delays appear to be a suitable solution because of the advantages of broad bandwidth, continuous tunability, and potential of large-scale integration. Based on this idea, the work on the development of an ORR-based photonic beam former systems for receiving PAAs have been carried out in the SMart Antenna system for Radio Transceivers (SMART) project and the Broadband Photonic Beamformer (BPB) project under SenterNovem project numbers IS053030 and IS052081, respectively.

In the SMART project an ORR-based beam former needs to be developed to control an 8-element linear array antenna (FLY antenna) which is designed to receive 10.75-12.7 GHz Ku-band satellite signals for aeronautical communication systems. In the BPB project an ORR-based beam former needs to be developed to control a 
$4 \times 4$ 2-D PAA-based radio telescope (SKY antenna) which is designed to receive the radiation of celestial objects in the frequency range from 400 to $1600 \mathrm{MHz}$.

\subsection{Outline of the thesis}

The arrangement of this thesis is as follows: Chapter 2 explains the principles of ORR-based delay lines. Chapter 3 compares different architectures of the ORRbased OBFN. Chapter 4 shows the advanced signal processing techniques for the beam former system. Chapter 5 focuses on the realization of the beam former chips. Chapter 6 introduces the beam former control system. Chapter 7 presents the measurement results on the test chips with beam former components and the entire beam former, which is followed by a test on a beam former demonstrator. At the end a summary and future perspectives are reviewed in Chapter 8 .

\section{References}

[1] "Satellite industry outlook-Beyond the global ecomonic downturn," ITU News, www.itu.int, issue Nov. 2009.

[2] Kristen Rohlfs, Thomas L. Wilson, Tools of Radio Astronomy. Springer 2003.

[3] Davis K. Cheng, Field and Wave Electomagnetics, $2^{\text {nd }}$ edition, Addison-Wesley publishing company, 1989.

[4] Constantine A. Balanis, Modern antenna handbook, John Wiley \& Sons, Inc, 2008.

[5] A. J. Seeds, "Microwave photonics," IEEE Trans. on Microwave Theory Tech. 50, 877-887 (2002).

[6] A. J. Seeds, C. H. Lee, E. Funk, and M. Nagamura, "Guest editorial: Microwave photonics," J. Lightwave Technol. 21, 2959-2960 (2003).

[7] A. J. Seeds, "Microwave photonics combines two worlds," Proc. IEEE Int. Topical Meeting Microwave Photon. Oqunquit, Maine, USA 16-19 (2004).

[8] S. A. Pappert, R. D. Esman, and B. Krantz, "Photonics for RF systems," in Proc. IEEE Avionics, Fiber-Optics, and Photonics Technology Conf., San Diego, CA, Sept. 2008, pp. 5-6.

[9] Masatoshi Shimizu, "Optical Access Network Technology Towards Expansion of FTTH," NTT Tsukuba Forum 2007 Workshop Lecture, Tsukuba, Japan, Oct. 2007.

[10] M. S. Alfiad, et al., "111 Gb/s POLMUX-RZ-DQPSK Transmission over 1140 $\mathrm{km}$ of SSMF with $10.7 \mathrm{~Gb} / \mathrm{s}$ NRZ-OOK Neighbours," Proceedings ECOC 2008, pp. Mo.4.E.2., Brussel, Belgium, Sept. 2008.

[11] G. Grosskopf et al., "Photonic 60-GHz maximum directivity beam former for smart antennas in mobile broad-band communications," IEEE Photon. Technol. Lett., vol. 14, no. 8, pp. 1169-1171, Aug. 2002. 
[12] M. A. Piqueras et al., "Optically beamformed beam-switched adaptive antennas for fixed and mobile broad-band wireless access networks," IEEE Trans.on Microwave Theory Tech. vol. 54, no. 2, pp. 887-899, Feb. 2006.

[13] J. L. Corral et al., "Dispersion-induced bandwidth limitation of variable true time delay lines based on linearly chirped fiber gratings," Electron. Lett., vol. 34, no. 2, pp. 209-211, Jan. 1998. 
Chapter 2

\section{Optical ring resonator-based delay lines}

\subsection{Introduction}

As mentioned in the previous section, in a photonic broadband beam former system the RF signals received by the AEs are modulated on optical carriers, and tunable optical delay lines synchronize the signals modulated on the optical carrier. In this study $1 \times 1$ (one input one output) optical ring resonators (ORRs) are used to implement the tunable optical delay lines. This chapter covers the principles of ORRs. First, transfer function and transfer matrix concepts are used to derive the frequency responses of ORRs. Next, delay properties of single and multiple cascaded ORRs are analyzed.

\subsection{Structure of ORR}

A $1 \times 1$ single-stage ORR consists of a ring-shaped waveguide and a straight waveguide which are able to couple light between each other. A schematic of a single ORR is shown Figure 2.1, where the power coupling coefficient $\kappa=[0 \cdots 1]$ represents the percentage of coupling, and $L_{R}$ is the roundtrip length of "ring".

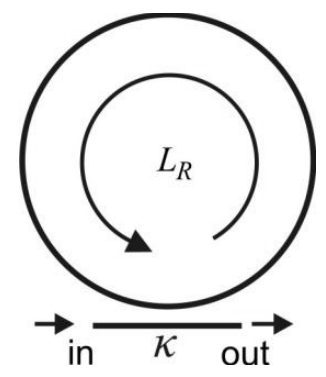

Figure 2.1: Structure of a $1 \times 1$ single-stage ORR

\subsection{Mathematical model of the ORR}

The behavior of an optical component can be described by its transfer function (or transfer matrix when a component has multiple input/output ports), which relates the amplitude and phase of the field at input to those at output. To derive the transfer 
function of an ORR, one can first look at the behavior of its two basic building blocks, namely a waveguide feedback path (ring) and a $2 \times 2$ coupler, which are shown in Figure 2.2.

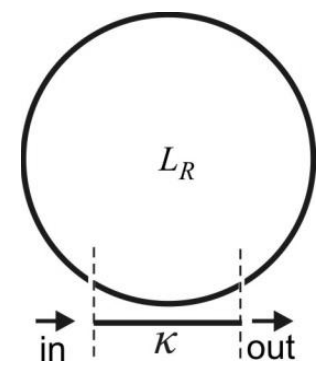

Figure 2.2: An ORR formed by a $2 \times 2$ coupler and a feedback waveguide

\subsubsection{Transfer function of waveguide feedback path}

The feedback waveguide path of an ORR can be regarded as a $1 \times 1$ transmission line. Consider, $E^{i}$ and $E^{o}$ are used to represent the complex amplitudes of the electric fields at input and output of the waveguide, then the relation between these two fields can be written as

$$
E^{o}=H_{L} \cdot E^{i}
$$

where $H_{L}$ represents the complex transfer function. If the waveguide has a length $L$, attenuation coefficient $\alpha$, and effective index $n_{\text {eff }}(\omega)$ [1], then $H_{L}$ can be written as

$$
H_{L}(\omega)=e^{-\alpha L} e^{-j \frac{\omega}{c_{0}} n_{e f f}(\omega) L}=r \cdot e^{-j \phi_{H_{L}}(\omega)}
$$

where $c_{0}$ is the speed of light in vacuum, $r=e^{-\alpha L}=[0, \ldots, 1]$ is defined as the amplitude transmission factor, and $\phi_{H_{L}}(\omega)=\omega \cdot n_{e f f}(\omega) L / c_{0}$ is the phase of the transfer function. The waveguide propagation loss $P_{l}$ in $\mathrm{dB} / \mathrm{cm}$ can be calculated by

$$
P_{l}=20 \log \left(e^{\alpha}\right)=20 \alpha \cdot \log (e)=8.686 \alpha
$$

When a frequency variation $\Delta \omega$ causes a phase shift $\Delta \phi_{H_{L}}$ of $2 \pi$, one can obtain the equation

$$
\begin{aligned}
\Delta \phi_{H_{L}} & =\phi_{H_{L}}\left(\omega_{0}+\Delta \omega\right)-\phi_{H_{L}}\left(\omega_{0}\right) \\
& =\frac{\omega_{0}+\Delta \omega}{c_{0}} n_{e f f}\left(\omega_{0}+\Delta \omega\right) L-\frac{\omega_{0}}{c_{0}} n_{e f f}\left(\omega_{0}\right) L=2 \pi
\end{aligned}
$$


In this thesis, the RF frequency range under study is in the order of tens of gigahertz, which is modulated on the optical carrier at $\lambda=1550 \mathrm{~nm}$ corresponding to $193 \mathrm{THz}$. So, one can consider that $\Delta \omega \ll \omega_{0}$. Then, Taylor approximation [1] can be used to express $n_{e f f}\left(\omega_{0}+\Delta \omega\right)$, which is given by

$$
\begin{aligned}
n_{e f f}\left(\omega_{0}+\Delta \omega\right) & =n_{e f f}\left(\omega_{0}\right)+\left.\frac{d n_{e f f}(\omega)}{d \omega}\right|_{\omega_{0}} \cdot \Delta \omega+\left.\frac{d^{2} n_{e f f}(\omega)}{d \omega}\right|_{\omega_{0}} \cdot \Delta \omega^{2}+\ldots \\
& \approx n_{e f f}\left(\omega_{0}\right)+\left.\frac{d n_{e f f}(\omega)}{d \omega}\right|_{\omega_{0}} \cdot \Delta \omega
\end{aligned}
$$

Then by inserting Eq. (2.5) in Eq. (2.4), one can obtain the equation

$$
\frac{\omega_{0}+\Delta \omega}{c_{0}}\left(n_{e f f}\left(\omega_{0}\right)+\left.\frac{d n_{e f f}(\omega)}{d \omega}\right|_{\omega_{0}} \cdot \Delta \omega\right) L-\frac{\omega_{0}}{c_{0}} n_{e f f}\left(\omega_{0}\right) L=2 \pi
$$

Further, Eq. (2.6) can be transformed into the following form:

$$
\Delta \omega=\frac{2 \pi c_{0}}{\left(n_{e f f}\left(\omega_{0}\right)+\left.\omega_{0} \cdot \frac{d n_{e f f}(\omega)}{d \omega}\right|_{\omega_{0}}\right) L}=\frac{2 \pi c_{0}}{n_{g} \cdot L}=2 \pi \Delta f_{F S R}=2 \pi / T
$$

where $n_{g}$ is the group index of the waveguide, $\Delta f_{F S R}$ is the free spectral range (FSR) of $H_{L}$, and $T$ is the group delay of the waveguide. The relation between $n_{g}$ and $n_{e f f}$ is expressed by

$$
n_{g}=n_{e f f}\left(\omega_{0}\right)+\left.\omega_{0} \cdot \frac{d n_{e f f}(\omega)}{d \omega}\right|_{\omega_{0}}
$$

Further, the group delay of the waveguide $T$ can be calculated by

$$
T=\frac{1}{\Delta f_{F S R}}=\frac{n_{g} \cdot L}{c_{0}}
$$

Then $H_{L}(\omega)$ can be written in terms of $T$ as

$$
H_{L}(\omega)=r \cdot e^{-j \omega T}
$$

To simplify the periodic frequency response, $\omega T$ can be replaced by $\Omega$ which represents the angular frequency normalized to the FSR: $\Omega=\omega / \Delta f_{\mathrm{FSR}}=\omega T$. Then $e^{-j \omega T}$ becomes $e^{-j \Omega}$, and $H_{L}(\Omega)$ is periodic with period $2 \pi$. Furthermore, $e^{-j \Omega}$ can be replaced by $z^{-1}$. This is the well-known z-transform, which is widely used in digital filter theory [2] and where $z^{-1}$ is used as the representation of a unit delay. Then, a delay-based signal filtering process can be expressed by a polynomial of 
$z^{-1}$, which simplifies the analyses of the filtering process. The z-transform of the $1 \times 1$ waveguide transmission line and its schematic drawing are given in Eq. (2.11) and Figure 2.3.

$$
H_{L}(z)=r e^{-j \phi_{Z}}{ }^{-1}
$$

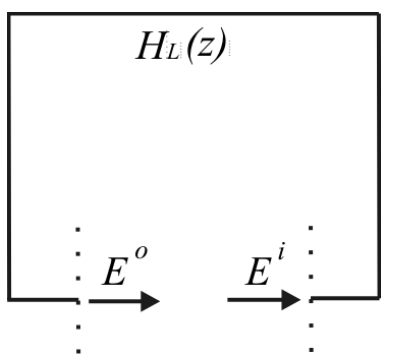

Figure 2.3: Schematic drawing of the $\mathrm{z}$-transform of $1 \times 1$ waveguide transmission line (reflections in the system are neglected)

\subsubsection{Transfer matrix of the $2 \times 2$ optical coupler}

A $2 \times 2$ optical coupler has two input ports and two output ports. Consider, for example, $E_{n}^{i}$ and $E_{n}^{o}(n=1,2)$ represent the complex amplitudes of the electric fields at input and output plane, respectively, as shown in Figure 2.4.

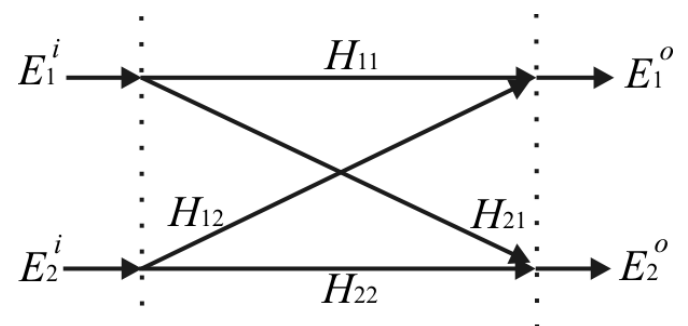

Figure 2.4: Schematic drawing of the possible transfers in a $2 \times 2$ optical coupler (reflections in the system are neglected)

Then the relation of the fields between input and output plane is given by

$$
\left[\begin{array}{l}
E_{1}^{o} \\
E_{2}^{o}
\end{array}\right]=\mathbf{H}\left[\begin{array}{l}
E_{1}^{i} \\
E_{2}^{i}
\end{array}\right]=\left[\begin{array}{ll}
H_{11} & H_{12} \\
H_{21} & H_{22}
\end{array}\right]\left[\begin{array}{l}
E_{1}^{i} \\
E_{2}^{i}
\end{array}\right]
$$

where the complex matrix $\mathbf{H}$ is the transfer matrix. It has two bar-transfer functions $H_{11}, H_{22}$ and two cross-transfer functions $H_{12}, H_{21}$. The transfer matrix $\mathbf{H}_{\text {tot }}$ of a composite device which is a concatenation of several elementary devices with individual transfer matrices $\mathbf{H}_{1}, \mathbf{H}_{2}, \ldots, \mathbf{H}_{\mathrm{n}-1}, \mathbf{H}_{\mathrm{n}}$ is written as 


$$
\mathbf{H}_{\mathrm{tot}}=\mathbf{H}_{\mathrm{n}} \mathbf{H}_{\mathrm{n}-1} \cdots \mathbf{H}_{2} \mathbf{H}_{1}
$$

A basic component of a $2 \times 2$ optical coupler is a directional coupler, as shown in Figure 2.5.

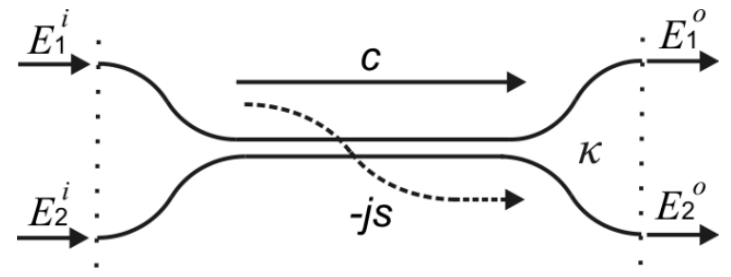

Figure 2.5: Implementation of $2 \times 2$ optical coupler with a directional coupler

Here, $c=\sqrt{1-\kappa}$ and $-j s=-j \sqrt{\kappa}$ are defined as bar-port and cross-port transmission coefficients, respectively, $\kappa$ is the power coupling coefficient. Omitting both the constant factor representing the average delay and possible loss, the transfer matrix of a directional coupler is given by

$$
\mathbf{H}_{\mathrm{dc}}=\left[\begin{array}{cc}
c & -j s \\
-j S & c
\end{array}\right]=\left[\begin{array}{cc}
\sqrt{1-\kappa} & -j \sqrt{\kappa} \\
-j \sqrt{\kappa} & \sqrt{1-\kappa}
\end{array}\right]
$$

Since loss is ignored here, the sum of the output powers equals the sum of the input powers by power conservation.

Another well-known $2 \times 2$ optical coupler is a symmetrical Mach-Zehnder Interferometer (MZI), as shown in Figure 2.6.

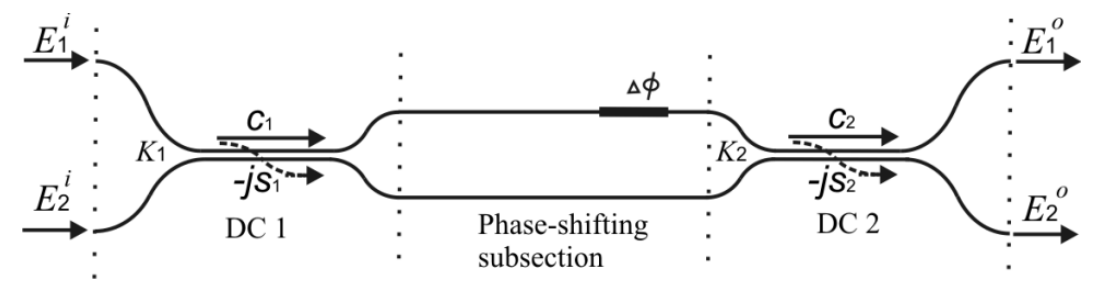

Figure 2.6: Implementation of $2 \times 2$ optical coupler with an MZI

It consists of 3 subsections, namely two directional couplers and a phase-shifting subsection in-between with two arms (signal paths) of equal length. The total transfer matrix of the MZI is calculated by multiplying the three individual matrices. Consider, for example, $c_{n}=\sqrt{1-\kappa_{n}}$ and $-j s_{n}=-j \sqrt{\kappa_{n}}$ are the bar-port and crossport transmission coefficients of directional coupler $n(n=1,2), \Delta \phi$ is the phase 
shift difference between the light in the two arms of the second subsection due to a small deviation from $n_{e}$ in the upper arm. Again, omitting the constant factor representing the average delay and possible loss, then the transfer matrix of the MZI is given by

$$
\begin{aligned}
\mathbf{H}_{\mathrm{MZI}} & =\left[\begin{array}{cc}
c_{2} & -j s_{2} \\
-j s_{2} & c_{2}
\end{array}\right]\left[\begin{array}{cc}
e^{-j \Delta \phi} & 0 \\
0 & 1
\end{array}\right]\left[\begin{array}{cc}
c_{1} & -j s_{1} \\
-j s_{1} & c_{1}
\end{array}\right] \\
& =\left[\begin{array}{cc}
-S_{1} s_{2}+c_{1} c_{2} e^{-j \Delta \phi} & -j\left(c_{1} s_{2}+s_{1} c_{2} e^{-j \Delta \phi}\right) \\
-j\left(-S_{1} c_{2}+c_{1} S_{2} e^{-j \Delta \phi}\right) & -c_{1} c_{2}+s_{1} s_{2} e^{-j \Delta \phi}
\end{array}\right]=\left[\begin{array}{ll}
H_{11} & H_{12} \\
H_{21} & H_{22}
\end{array}\right]
\end{aligned}
$$

In practice the two couplers in the MZI can be considered identical: $\kappa_{1}=\kappa_{2}=\kappa_{\mathrm{DC}}$, $\left(\kappa_{\mathrm{DC}}\right.$ is a constant value in the range $\left.[0,1]\right)$. Insert this into Eq. $(2.15)$, then the power coupling coefficient $\kappa_{\mathrm{MZI}}$ is written as

$$
\kappa_{\mathrm{MZI}}=\left|H_{12}\right|^{2}=\left|H_{21}\right|^{2}=4 \kappa_{\mathrm{DC}}\left(1-\kappa_{\mathrm{DC}}\right) \cos ^{2}\left(\frac{\Delta \phi}{2}\right)
$$

Eq. (2.16) shows that by changing $\Delta \phi$ the value of $\kappa_{\mathrm{MZI}}$ can be tuned in the range $\left[0,4 \kappa_{\mathrm{DC}}\left(1-\kappa_{\mathrm{DC}}\right)\right]$. Figure 2.7 shows the tuning of $\kappa_{\mathrm{MZI}}$ for different values of $\kappa_{\mathrm{DC}}$. As the complimentary effect, the tuning range of the bar-port power transmission ratio $\left|H_{11}\right|^{2}$ or $\left|H_{22}\right|^{2}$ is then $\left[1-4 \kappa_{\mathrm{DC}}\left(1-\kappa_{\mathrm{DC}}\right), 1\right]$. It can be calculated that when $\kappa_{\mathrm{DC}}=0.5$, the MZI is able to reach the ideal power coupling tuning range $[0,1]$. Thus, in our application symmetrical MZIs are used as tunable couplers of the ORRs and $\Delta \phi$ is added intentionally for the tuning purpose. Moreover, since loss is not considered here, the sum of the output powers equals the sum of the input powers by power conservation.

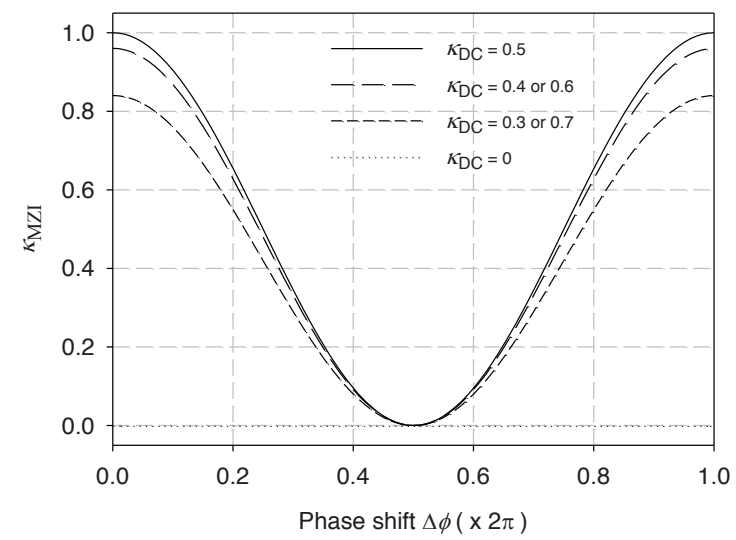

Figure 2.7: Tuning of $\kappa_{\mathrm{MZI}}$ for different values of $\kappa_{\mathrm{DC}}$ 


\subsubsection{The transfer function of the ORR}

With the knowledge of the z-transform of a waveguide transmission line and the transfer matrix $\mathbf{H}$ of a $2 \times 2$ optical coupler, the z-transform schematic of an ORR can be obtained as shown in Figure. 2.8.

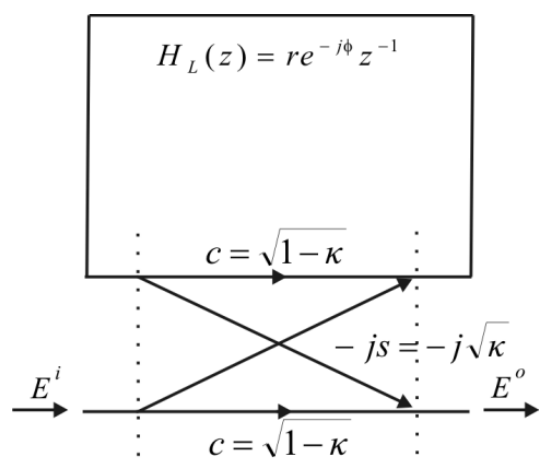

Figure 2.8: $z$-transform schematic of an ORR

Following the paths, the transfer function of the ORR $H(z)$ can be derived by

$$
\begin{aligned}
H(z) & =c-s^{2} r z^{-1} e^{-j \phi}\left[1+c r z^{-1} e^{-j \phi}+\left(c r z^{-1} e^{-j \phi}\right)^{2}+\cdots\right] \\
& =\frac{c-r z^{-1} e^{-j \phi}}{1-c r z^{-1} e^{-j \phi}}=\frac{\sqrt{1-\kappa}-r z^{-1} e^{-j \phi}}{1-\sqrt{1-\kappa} r z^{-1} e^{-j \phi}}
\end{aligned}
$$

The frequency response of the ORR in normalized angular frequency can be obtained from Eq. (2.17) by substituting $e^{-j \Omega}$ for $z^{-1}$, which is written as

$$
H(\Omega)=\frac{\sqrt{1-\kappa}-r e^{-j \Omega} e^{-j \phi}}{1-\sqrt{1-\kappa} r e^{-j \Omega} e^{-j \phi}}=\frac{\sqrt{1-\kappa}-r e^{-j \omega T} e^{-j \phi}}{1-\sqrt{1-\kappa} r e^{-j \omega T} e^{-j \phi}}
$$

To show the delay characteristics of the ORR, the concept of group delay is used, which is defined as the negative derivative of the phase of the transfer function with respect to the frequency [2]. In case the normalized angular frequency is used, the normalized group delay $\tau$ can be calculated by

$$
\tau=-\frac{d \Psi(\Omega)}{d \Omega}=-\frac{d}{d \Omega} \arctan \left[\frac{\operatorname{Im}\{H(\Omega)\}}{\operatorname{Re}\{H(\Omega)\}}\right]
$$

where $\tau$ is normalized to the roundtrip delay $T$. The absolute group delay can then be calculated by

$$
\tau_{g}=T \tau
$$




\subsubsection{Time domain analysis}

An ORR is modeled as a linear time-invariant system, which can also be characterized in the time domain by its impulse response $h(t)$ [3]. If $x(t)$ and $y(t)$ are used to represent the continuous-time input and output signal of the ORR, respectively, then their time-domain relation is given by

$$
y(t)=h(t) * x(t)=\int_{-\infty}^{\infty} x(\tau) h(t-\tau) d \tau
$$

The corresponding frequency domain relation is given by

$$
Y(\omega)=H(\omega) X(\omega)
$$

where $Y(\omega)$ and $X(\omega)$ are the Fourier transforms of $y(t)$ and $x(t)$, respectively, and $H(\omega)$ represents the frequency response of the ORR. When $H(\omega)$ is given, $h(t)$ can also be derived by inverse Fourier transform as

$$
h(t)=\frac{1}{2 \pi} \int_{-\infty}^{\infty} H(\omega) e^{j \omega t} d \omega
$$

Then by inserting Eq. (2.18) into Eq. (2.23), the impulse response of an ORR can be written as

$$
h(t)=c \delta(t)-s^{2} r \sum_{n=0}^{\infty} c^{n} r^{n} \delta[t-(n+1) T]
$$

where $\delta(t)$ is the impulse (delta) function [2]. Eq. (2.24) shows that an ORR has a discrete-time infinite impulse response (IIR) with delays integer multiples of $T$. This is because an ORR is a feedback system and each roundtrip in the ORR introduces a delay $T$ [4]. An example of the impulse response of an ORR with lossless waveguide and $3 \mathrm{~dB}$ coupling $(r=1, \kappa=0.5, c=s=\sqrt{2} / 2)$ is shown in Figure 2.9.

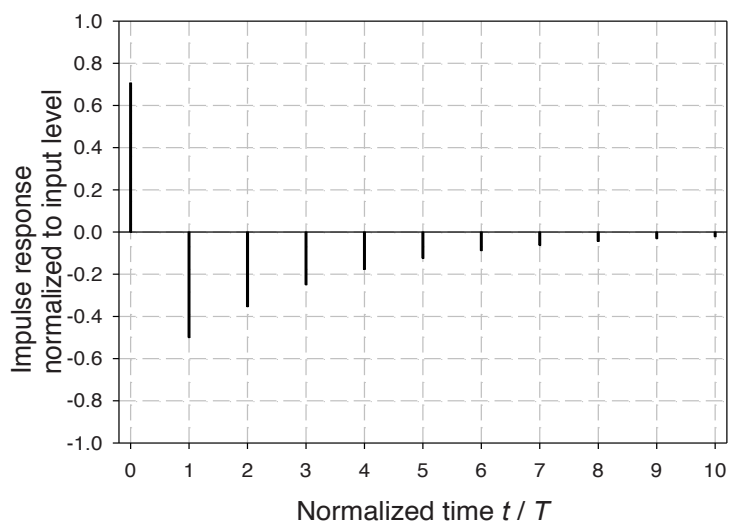

Figure 2.9: Impulse response of a lossless ORR with $3 \mathrm{~dB}$ coupling 
Apparently, an ORR can be regarded as a discrete-time IIR filter, whose delays are integer multiples of a unit delay that is the smallest delay difference between different signal paths. In case of an ORR the unit delay is the roundtrip delay time $T$. Changing to the discrete domain, the delta function $\delta(t)$ in Eq. (2.24) can be replaced by the Kronecker delta function $\delta(k)$ [2], which gives

$$
h(k)=c \delta(k)-s^{2} r \sum_{n=0}^{\infty} c^{n} r^{n} \delta(k-1-n)
$$

Now, the ORR is modeled as an IIR digital filter, which means that the well-known digital filter concepts can be used to analyze the properties of an ORR.

\subsubsection{Optical all-pass filter}

An ideal lossless ORR can be regarded as an optical all-pass filter [5]. The frequency response of an all-pass filter is given by

$$
H_{A}(\omega)=e^{j \Psi(\omega)}
$$

It has a unity magnitude response $\left|H_{A}(\omega)\right|=1$ and its phase or group delay can be tailored to approximate any desired response. In the digital domain, the z-transform of an all-pass filter can be formulated in a ratio between two polynomials [5]:

$$
H_{A}(z)=e^{j \zeta} \frac{d_{N}^{*}+d_{N-1}^{*} z^{-1}+\cdots+z^{-N}}{1+d_{1} z^{-1}+\cdots+d_{N} z^{-N}}=e^{j \zeta} \frac{D_{N}^{R}(z)}{D_{N}(z)}
$$

The coefficients in the numerator can be determined directly from those in the denominator by reversing the order of its coefficients and taking their complex conjugate. Here, $D_{N}(z)$ and $D_{N}^{R}(z)$ are used to represent such a polynomial pair. A constant phase factor, $\zeta$, is included for generality, which does not change the magnitude nor the group delay response. For an ideal lossless ORR, its transmission coefficient $r=1$, and then its z-transform $H_{\text {lossless }}(z)$ can be written as

$$
H_{\text {lossless }}(z)=-e^{-j \phi} \frac{-\sqrt{1-\kappa} e^{+j \phi}+z^{-1}}{1-\sqrt{1-\kappa} e^{-j \phi_{Z^{-1}}}}=-e^{-j \phi} \frac{d^{*}+z^{-1}}{1+d z^{-1}}=-e^{-j \phi} \frac{D^{R}(z)}{D(z)}
$$

where $d=-\sqrt{1-\kappa} e^{-j \phi}$. This all-pass filter concept is also applicable for a cascade of ideal lossless ORRs, since the transfer function of a cascade is simply the product of those of the individual ORRs. Therefore, in practice a cascade of multiple low-loss ORRs plays a very similar role as an optical multi-stage all-pass filter and can be used to approximate any desired phase and group delay response. 


\subsection{Properties of ORR-based delay lines}

\subsubsection{Single ORR}

To understand the signal processing principles of an ORR, one should turn to the ORR frequency response that describes how each frequency component of the input signal is modified by the ORR. From Eq. (2.18) the magnitude response of an ORR is given by

$$
|H(\Omega)|=\sqrt{\frac{1-\kappa+r^{2}-2 r \sqrt{1-\kappa} \cos (\Omega+\phi)}{1+r^{2}(1-\kappa)-2 r \sqrt{1-\kappa} \cos (\Omega+\phi)}}
$$

or more commonly in the optical domain, the power transfer in $\mathrm{dB}$ is given by

$$
|H(\Omega)|_{\mathrm{dB}}^{2}=10 \log _{10}\left[\frac{1-\kappa+r^{2}-2 r \sqrt{1-\kappa} \cos (\Omega+\phi)}{1+r^{2}(1-\kappa)-2 r \sqrt{1-\kappa} \cos (\Omega+\phi)}\right]
$$

The phase response and the corresponding group delay response of the ORR are given by

$$
\begin{gathered}
\Psi(\Omega)=\arctan \left[\frac{\frac{r}{\sqrt{1-\kappa}} \sin (\Omega+\phi)}{1-\frac{r}{\sqrt{1-\kappa}} \cos (\Omega+\phi)}\right]-\arctan \left[\frac{r \sqrt{1-\kappa} \sin (\Omega+\phi)}{1-r \sqrt{1-\kappa} \cos (\Omega+\phi)}\right] \\
\tau(\Omega)=\left[\frac{\mathrm{r} \sqrt{1-\kappa} \cos (\Omega+\phi)-\mathrm{r}^{2} \sqrt{1-\kappa}}{1-2 \mathrm{r} \sqrt{1-\kappa} \cos (\Omega+\phi)+\mathrm{r}^{2} \sqrt{1-\kappa}}+\frac{\mathrm{r}^{2}-\mathrm{r} \sqrt{1-\kappa} \cos (\Omega+\phi)}{1-\kappa-2 \mathrm{r} \sqrt{1-\kappa} \cos (\Omega+\phi)+\mathrm{r}^{2}}\right]
\end{gathered}
$$

\section{Phase and group delay}

Figure 2.10 shows the phase and group delay responses for three different resonance frequencies and Figure 2.11 shows the responses for three different coupling coefficients. These numerical examples reveal the most important properties of an ORR when it is applied as an optical delay line. In these examples, the roundtrip loss $P_{L}=0.5 \mathrm{~dB}\left(r=10^{-P_{L} / 20} \approx 0.95\right)$ is used for the ORR to match the low-loss condition $P_{L}<1 \mathrm{~dB}$.

The frequency response of an ORR is controlled by the additional roundtrip phase shift $\phi$ and the power coupling ratio $\kappa$, which determine the offset of the resonance frequency and the shape of the phase/group delay response, respectively. When $\kappa=0$, no portion of input light couples to the ring, so the ORR generates zero phase shift and zero delay. When $\kappa=1$, the input light couples completely to the ring, and after one roundtrip couples completely out. In this case the ORR plays the same role as a $1 \times 1$ waveguide, so it has a linear phase transition of $2 \pi$ over one FSR and a 
flat group delay response with the value of the roundtrip delay. When $0<\kappa<1$, portion of the input light couples to the ring, and after each roundtrip a portion of the input couples out, so that the output consists of the contributions from different roundtrips. In this case the phase transition over one FSR is still $2 \pi$, but the phase

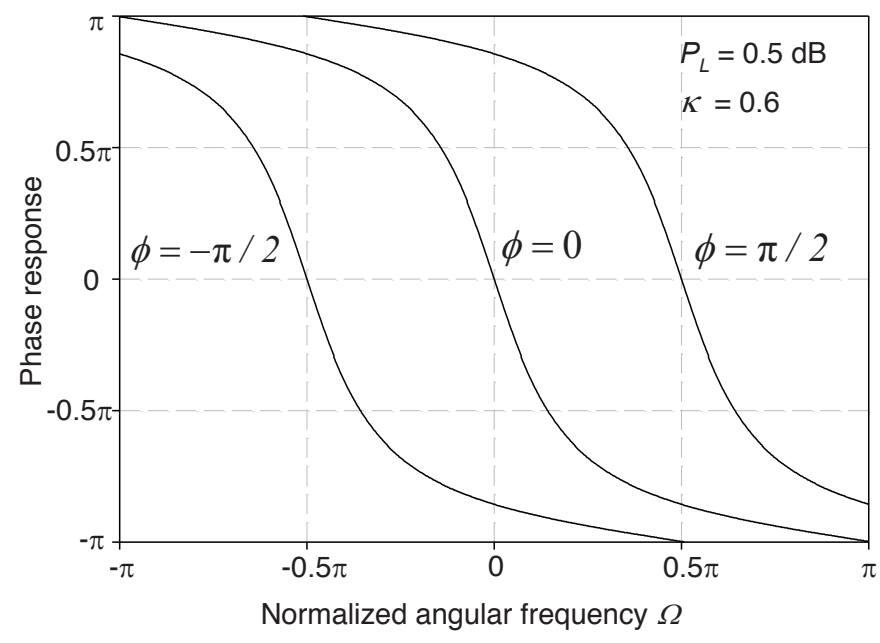

(a)

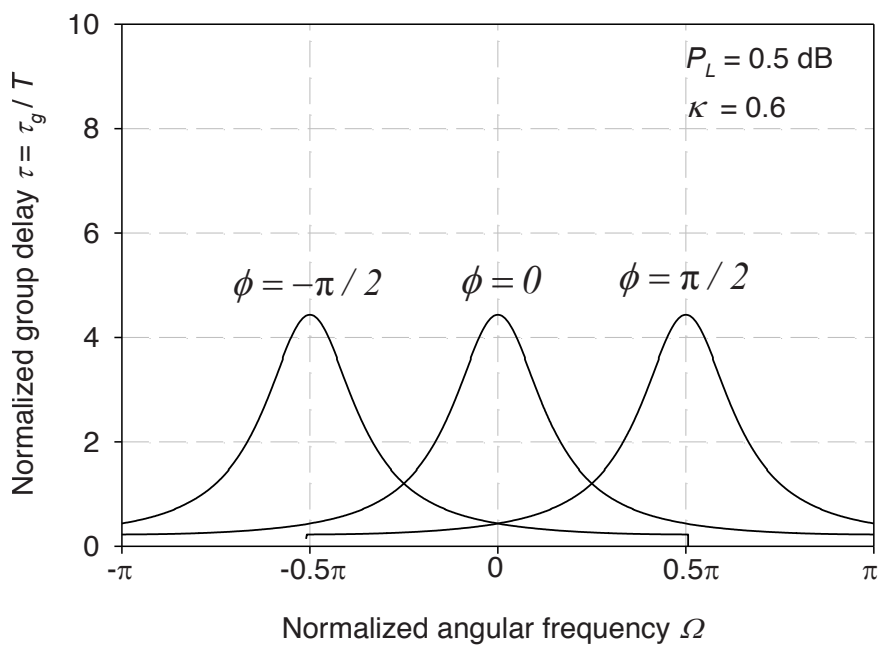

(b)

Figure 2.10: (a) phase responses of a single ORR for different values of $\phi$,

(b) group delay responses of a single ORR for different values of $\phi$. 
shift varies increasingly sharper as the corresponding frequency approaches the resonance frequency, which, for most values of $\kappa$, results in a descreasing phase response and a bell-shaped group delay response centered at the resonance frequency as shown in Figure 2.10 and 2.11

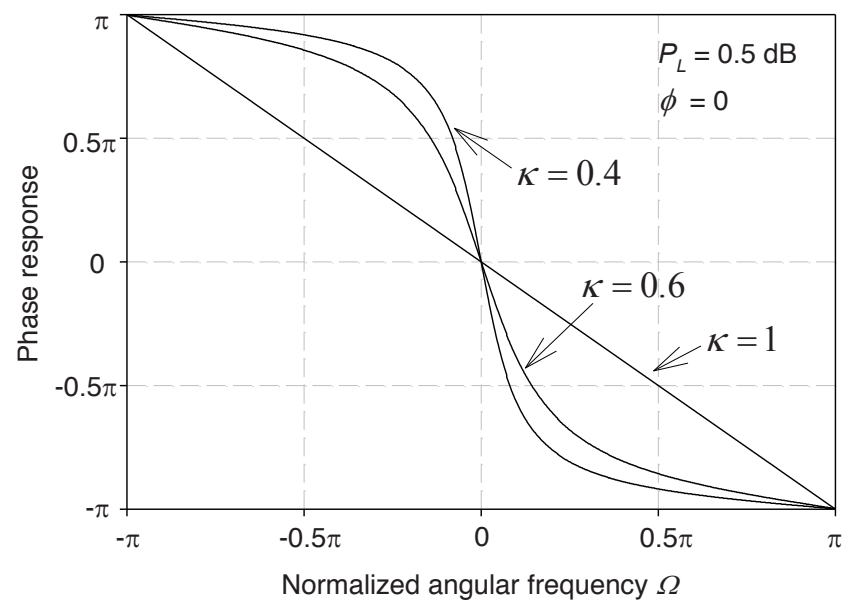

(a)

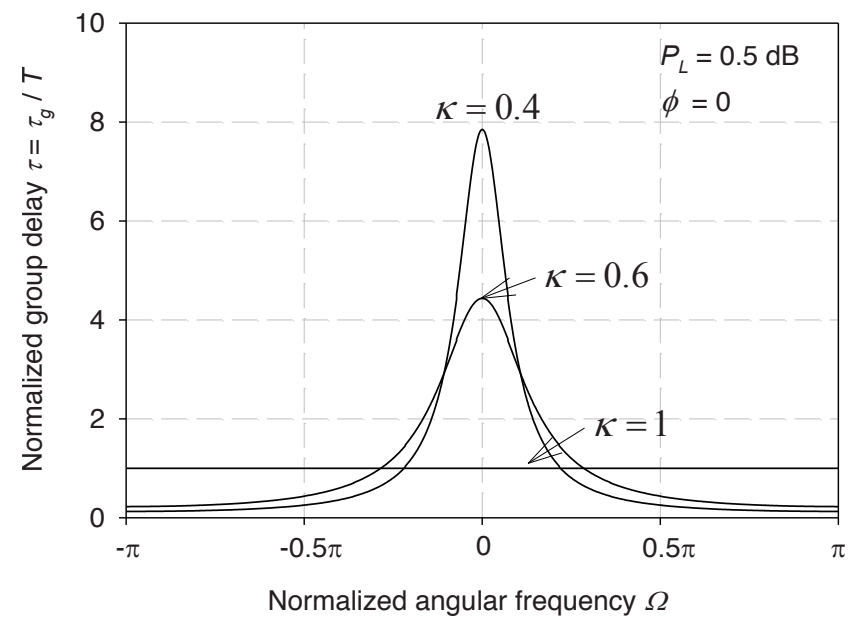

(b)

Figure 2.11: (a) phase responses of a single ORR for different values of $\kappa$,

(b) group delay responses of a single ORR for different values of $\kappa$. 
However, for such a lossy ORR, if $\kappa$ is lower than the so-called critical coupling point [6], [7], the abnormal phase response, which breaks the property of the decreasing phase response, will occur around the resonance frequency, resulting in negative group delay [6], [7]. Numerical examples showing the abnormal phase response and the corresponding negative group delay response is given in Figure 2.12. The range of $\kappa$ resulting in negative delay will be explained in the next section.

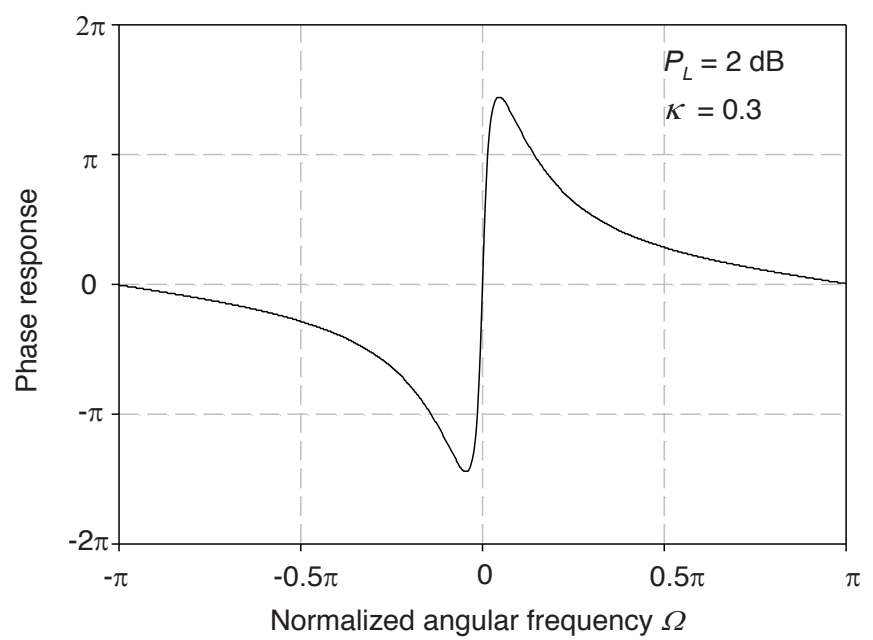

(a)

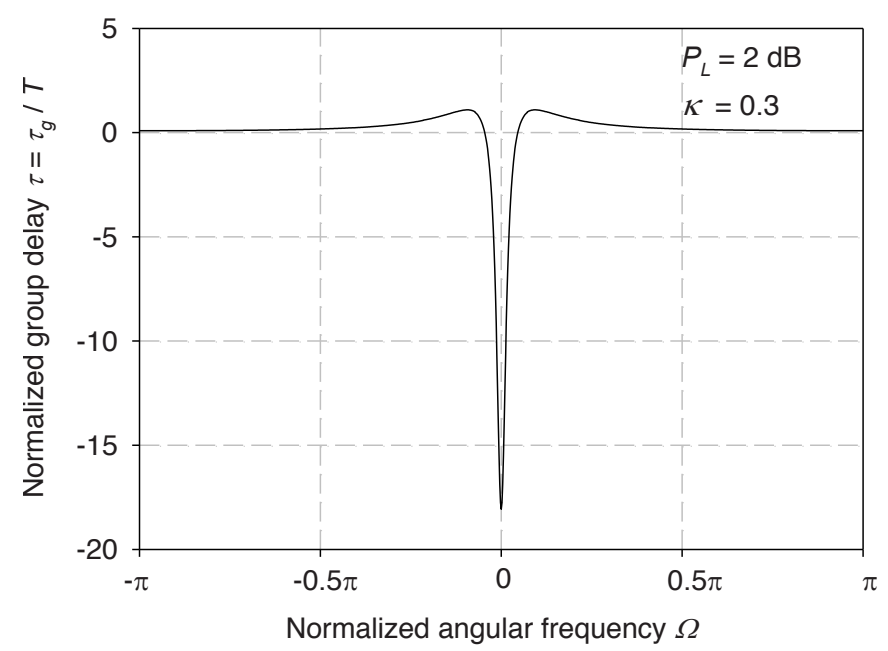

(b)

Figure 2.12: (a) phase response of a single ORR with abnormal phase shift, (b) group delay response of a single ORR with negative delay. 


\section{Tuning range}

The relation between $\kappa$ and normalized peak delay $\tau_{p}$ at the resonance frequency is obtained by setting $\Omega+\phi$ to 0 in Eq. (2.32):

$$
\tau_{p}(\kappa)=\frac{r \sqrt{1-\kappa}}{1-r \sqrt{1-\kappa}}+\frac{r}{r-\sqrt{1-\kappa}}
$$

which is illustrated in Figure 2.13 for different values of the roundtrip loss.

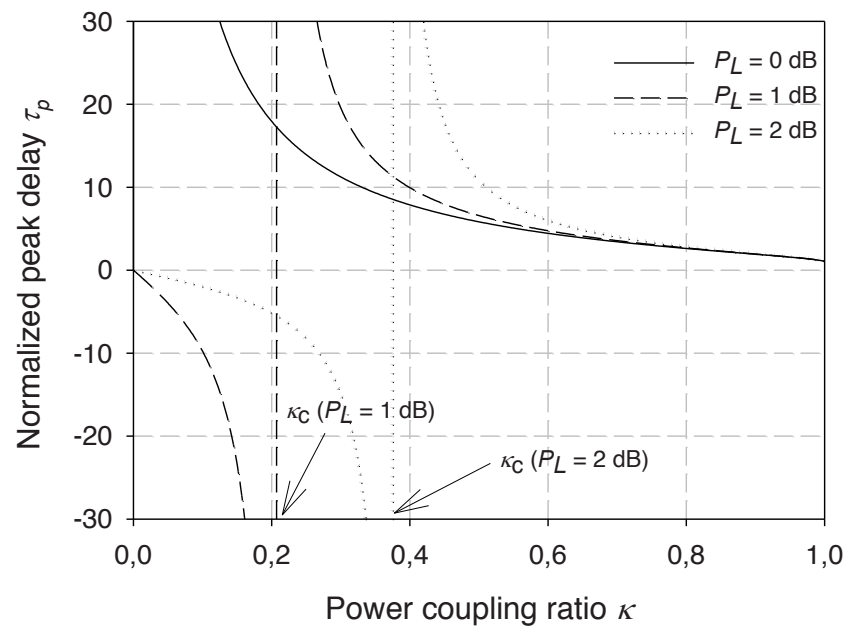

Figure 2.13: Peak delay value $\tau_{p}$ against $\kappa$ for different values of roundtrip loss $P_{L}$

Figure 2.12 illustrates that an ORR is able to generate higher delays than its unit delay, and $\tau_{p}$ increases from 1 to infinity as $\kappa$ decreases from 1 to the so-called critical coupling point $\kappa_{\mathrm{c}}$. From the second term of Eq. (2.33) it can be seen that the $\tau_{p}$ reaches infinity when $r-\sqrt{1-\kappa}$ equals zero $(r=c)$. Then the critical coupling point $\kappa_{c}$ can be calculated by:

$$
\kappa_{c}=1-r^{2}=1-10^{-P_{L} / 10}
$$

When $\kappa$ has a value lower than $\kappa_{c}$, the peak delay $\tau_{p}$ will have negative value as shown in Figure 2.12. The concept of the negative group delay is not in the scope of this thesis; therefore it will not be further discussed. The relevant information about the negative group delay can be found in [6], [7]. Here, we consider only the positive group delays generated by the ORR, and the practical operating range of $\kappa$ is $\left\langle\kappa_{\mathrm{c}}, 1\right]$. 


\section{Bandwidth-delay relation}

Figure 2.11 (b) shows that when $\tau_{p}$ increases, the width of the "bell" decreases. This occurs because the area under the group delay curve actually represents the phase shift of the ORR, which is a constant $2 \pi$ for one FSR. Note that this observation reveals the inherent tradeoff of an ORR between delay value and bandwidth. A single ORR has its delay band around the resonance frequency, and the definition of bandwidth is illustrated in Figure 2.14, where the delay band is defined as the frequency band between the two vertical dashed lines, $\Delta \Omega_{\mathrm{BW}}$ is the corresponding bandwidth, $\bar{\tau}$ is the average delay value over the delay band, and $\Delta \tau$ is the maximum delay ripple (maximum range of delay variation) in the delay band. Note that all these three quantities are normalized with respect to the FSR of the ORR.

Besides the inherent tradeoff between the delay and the bandwidth, the waveguide loss also has influence on this relation of an ORR, since the roundtrip loss $P_{L}$ of the ORR is also a factor determining the group delay response of the ORR ( $r=$ $10^{-P_{L} / 20}$ ). However, when the roundtrip loss is low, for example $P_{L}=1 \mathrm{~dB}$, the effect of the waveguide loss on the delay bandwidth is negligible, as illustrated in Figure 2.15. In this figure three group delay responses for different values of roundtrip loss $P_{L}$ are shown. These three group delay responses are tuned to have the same peak delay value $\tau_{p}=3$, and it can be seen that for $P_{L}=1 \mathrm{~dB}$ the corresponding group delay response is hardly different than that in the ideal lossless case.

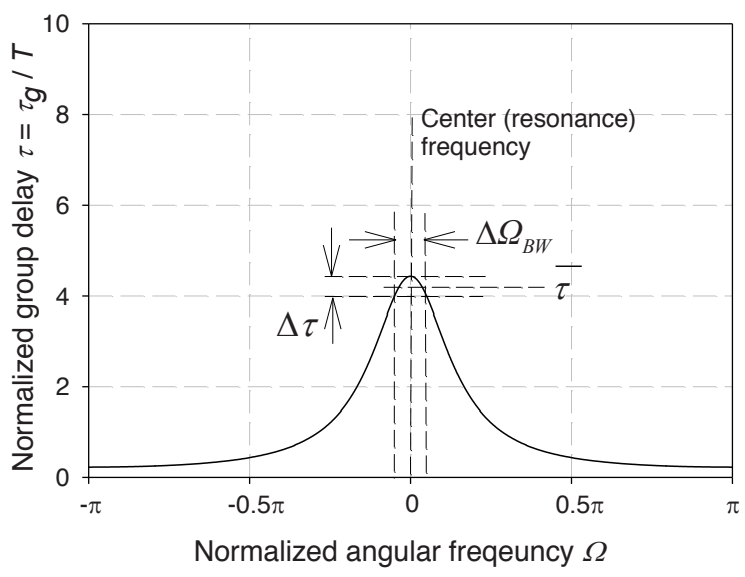

Figure 2.14: Definition of delay bandwidth of a single ORR 


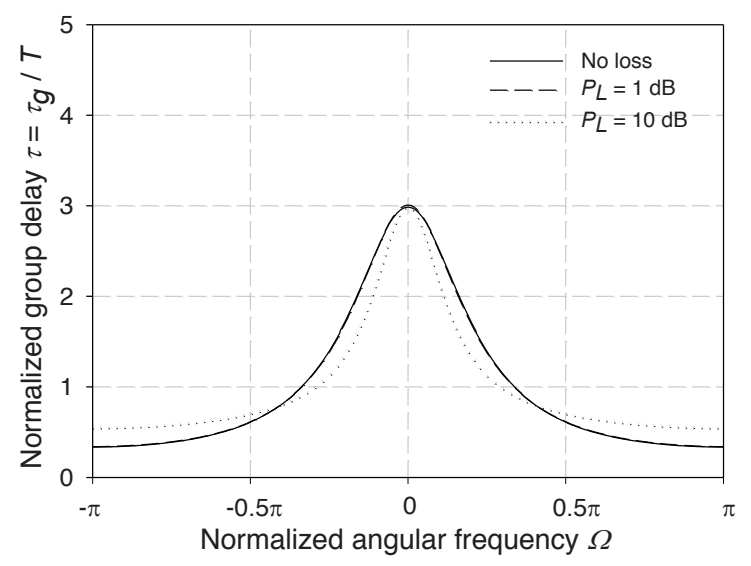

Figure 2.15: Group delay responses for different values of roundtrip loss $P_{L}$

\section{Roundtrip length-delay functionality relation}

It can be seen from the previous section that the bandwidth-delay relation of ORRs is actually independent of the FSR of the ORR since both quantities are normalized to the FSR, namely normalized group delay and normalized angular frequency. In other words, the roundtrip length/physical size of the ORR is not a determinative factor of the delay functionality of the ORR. To illustrate this property, a numerical example is shown in Figure 2.16, where the group delay responses of two lossless ORRs with different FSRs, $5 \mathrm{GHz}$ and $10 \mathrm{GHz}$, are compared on absolute time and frequency scale. Both group delay responses are tuned to have the same peak delay value of $5 \mathrm{~ns}$. It can be seen that for the delay band region the two ORRs have the same group delay responses.

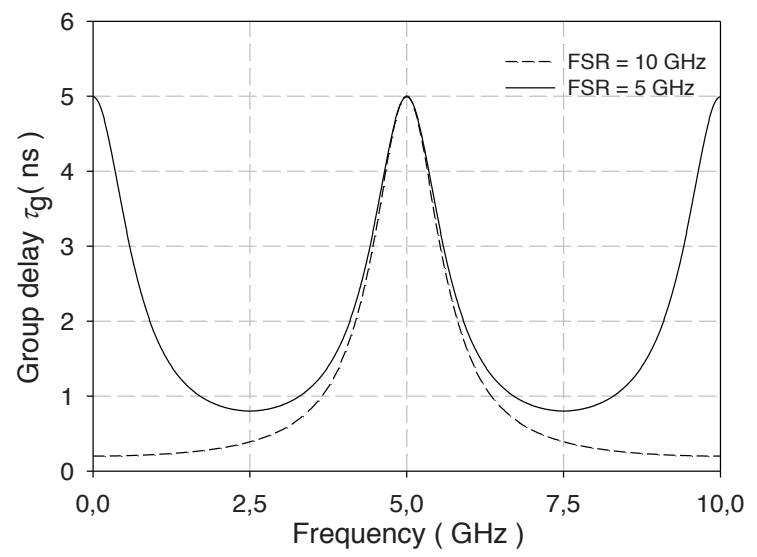

Figure 2.16: Group delay responses between two ORRs with different FSRs 


\section{Power transfer and loss}

After the illustration of phase and group delay response, now let us take a look at the power transfer of a single-stage ORR. Three numerical examples of group delay responses and the corresponding power transfers are shown in Figure 2.17. The normalized peak delays of the three group delay responses are set to the same value of 3. The group delay responses appear to overlap one another since the effect of the low roundtrip loss $\left(P_{L}<1 \mathrm{~dB}\right)$ on the group delay response of the ORR is negligible as explained in one of the previous sections. Compared to the group delay response, the power transfer of a lossy ORR has the same "bell" shape but an inverted version.

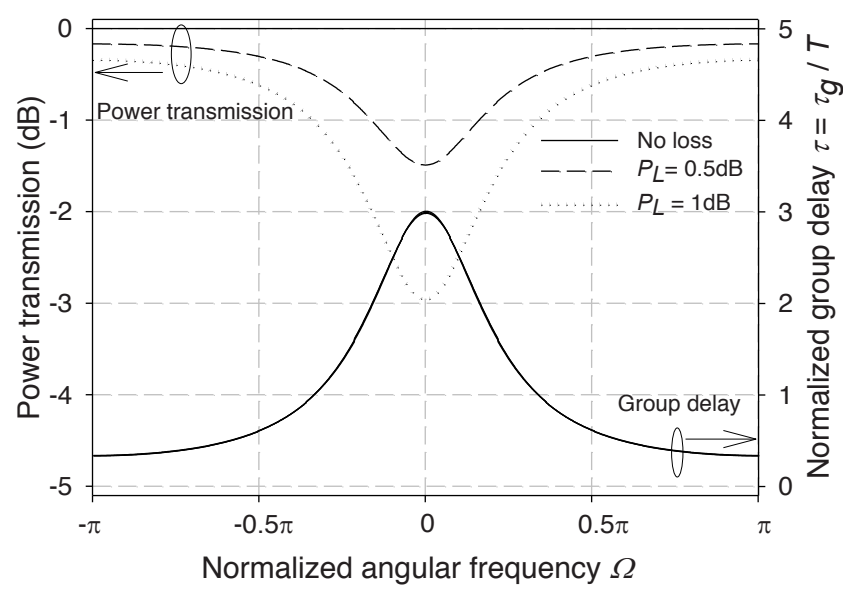

Figure 2.17: Group delay responses and the corresponding power transfers of a single-stage ORR for different values of the roundtrip loss

This is because higher group delay means longer distance which correspondingly gives higher loss. The relation between the loss at the resonance frequency $P_{L_{-} R}$ and the coupling coefficient $\kappa$ can be found from Eq. (2.30), which is given by

$$
P_{L_{-} R}(\kappa)=-10 \log _{10}\left[\frac{1-\kappa+r^{2}-2 r \sqrt{1-\kappa}}{1+r^{2}(1-\kappa)-2 r \sqrt{1-\kappa}}\right]
$$

Three numerical examples of this relation for three different values of the roundtrip $\operatorname{loss} P_{L}\left(r=10^{-P_{L} / 20}\right)$ are shown in Figure 2.18. It can be seen that when $\kappa=1$, the ORR behaves as a line waveguide, and therefore $P_{L_{-} R}=P_{L}$; when $\kappa$ decreases to the critical coupling point $\kappa_{\mathrm{c}}$, the delay value approaches infinity (see Eq. (2.33)), and therefore the delay distance generated by the ORR and the corresponding loss increases to infinity. 


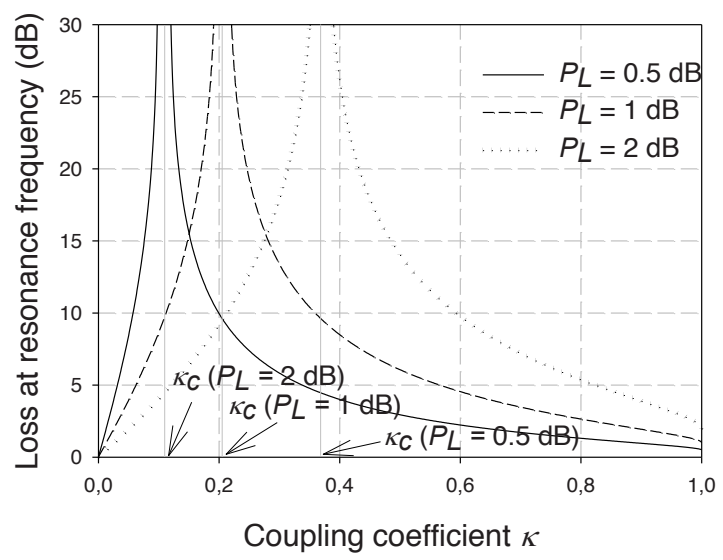

Figure 2.18: Relation between the loss at resonance frequency and coupling coefficient of the ORR for three different roundtrip losses.

Then, by combining Eq. (2.33) and Eq. (2.35), one can obtain the relation between the loss and delay value at the resonance frequency of the ORR, which is given by

$$
P_{L_{-} R}=-10 \log _{10}\left(\frac{2 r^{2}+\tau r^{2}-\tau+\sqrt{\tau^{2} r^{4}-2 \tau^{2} r^{2}+\tau^{2}+4 r^{2}}}{\tau r^{3}-\tau r-2 r-r \sqrt{\tau^{2} r^{4}-2 \tau^{2} r^{2}+\tau^{2}+4 r^{2}}}\right)^{2}
$$

Three examples for three different values of the roundtrip loss $P_{L}\left(r=10^{-P_{L} / 20}\right)$ are shown in Figure 2.19. Unlike in the line waveguide case, the relation between the loss and delay of the ORR appears to be nonlinear, as indicated in Eq. (2.36). This effect becomes more noticeable when the ORR has higher roundtrip loss $P_{L}$. However, for the low-loss case $\left(P_{L}<1 \mathrm{~dB}\right)$ this nonlinearity is not significant, and one can assume that the loss increases proportionally with the delay value.

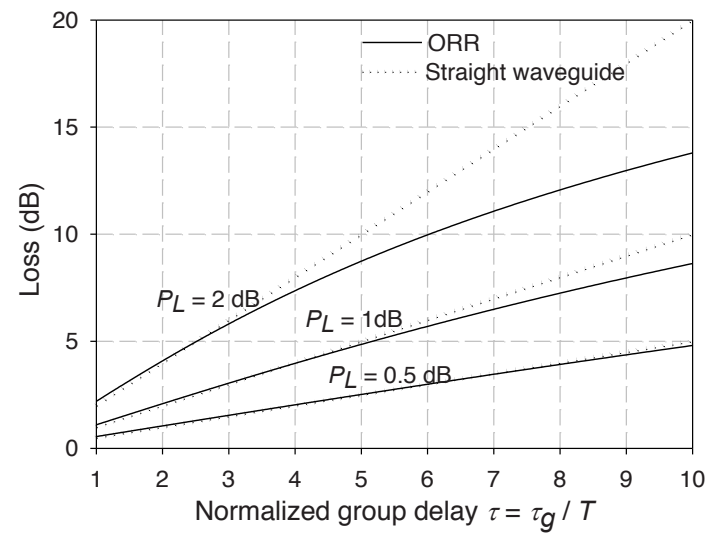

Figure 2.19: Relation between loss and group delay of an ORR for different $P_{L}$ 


\subsubsection{Cascade of multiple ORRs}

As shown in the previous section, the group delay response of an ORR becomes narrower as the peak delay value increases. When the required group delay value for the input signal is such that a single ORR cannot provide sufficient bandwidth, a cascade of multiple ORRs can be used to increase the delay bandwidth. The cascaded ORRs considered here are of the same FSR and have the same roundtrip loss. The transfer function of $\mathrm{N}$-stage cascaded ORRs in normalized angular frequency is the product of those of the individual single-stage ORRs:

$$
H(\Omega)=\prod_{i=1}^{N} H_{i}(\Omega)=\left(\prod_{i=1}^{N}\left|H_{i}(\Omega)\right|\right) e^{j \sum_{i=1}^{N} \Psi_{i}(\Omega)}
$$

where $H_{i}(\Omega)$ is the transfer function of the $i$ th stage. The corresponding magnitude, power transmission, phase, and group delay response are given by

$$
\begin{gathered}
|H(\Omega)|=\prod_{i=1}^{N}\left|H_{i}(\Omega)\right| \\
|H(\Omega)|_{\mathrm{dB}}^{2}=\sum_{i=1}^{N} 10 \log _{10}\left|H_{i}(\Omega)\right|^{2} \\
\Psi(\Omega)=\sum_{i=1}^{N} \Psi_{i}(\Omega) \\
\tau(\Omega)=-\frac{\mathrm{d} \Psi(\Omega)}{\mathrm{d} \Omega}=-\frac{\mathrm{d} \sum_{i=1}^{N} \Psi_{i}(\Omega)}{\mathrm{d} \Omega}=\sum_{i=1}^{N} \tau_{i}(\Omega)
\end{gathered}
$$

\section{Group delay}

By properly choosing $\phi_{i}$ and $\kappa_{i}$ of each individual ORR in the cascade, a flattened delay band can be generated for a desired group delay value, which has an increased bandwidth. An example of the delay characteristic of a cascade of three ORRs is shown in Figure 2.20.

It can be seen that the generated delay band is not completely flat but has a ripple. Similar to the variation over the delay band of the single ORR, the ripple will result in unequal delays to different frequency components of the signals which fall in the delay band. Then when ORRs are used in an OBFN as the delay elements, the ripples will lead to variations in the beam angle of the antenna [8]. For a single ORR, to reduce the delay variation over the delay band means to reduce the bandwidth (see Figure 2.14). For a cascade of multiple ORRs, the delay ripple can be reduced by means of the so-called squeezing method [9], which means shifting the resonance frequencies of the individual ORRs closer to each other. This way, the bandwidth is also reduced accordingly. An illustration of the squeezing method for a 2-stage ORR cascade is given in Figure 2.21. 

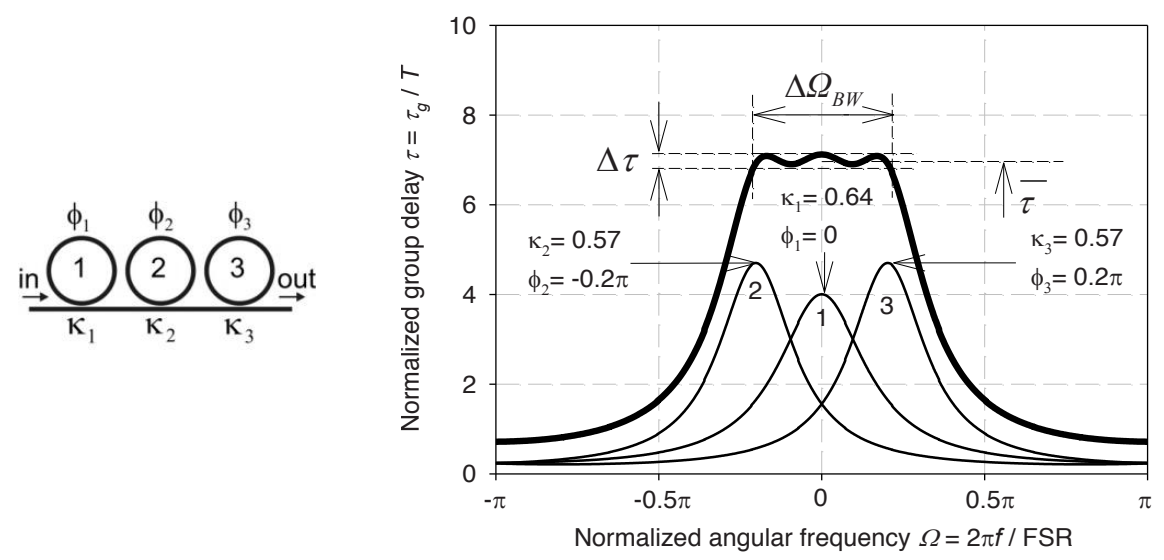

Figure 2.20: Group delay response of a cascade of three ORRs (thick) and the corresponding individual ORRs in the cascade (thin)

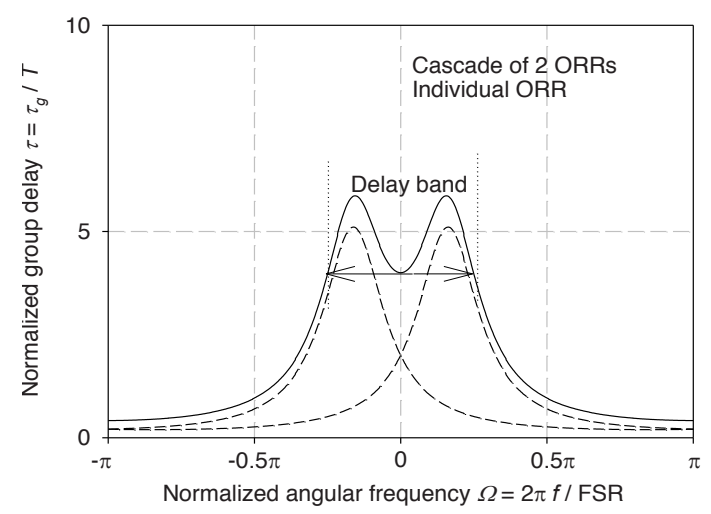

(a)

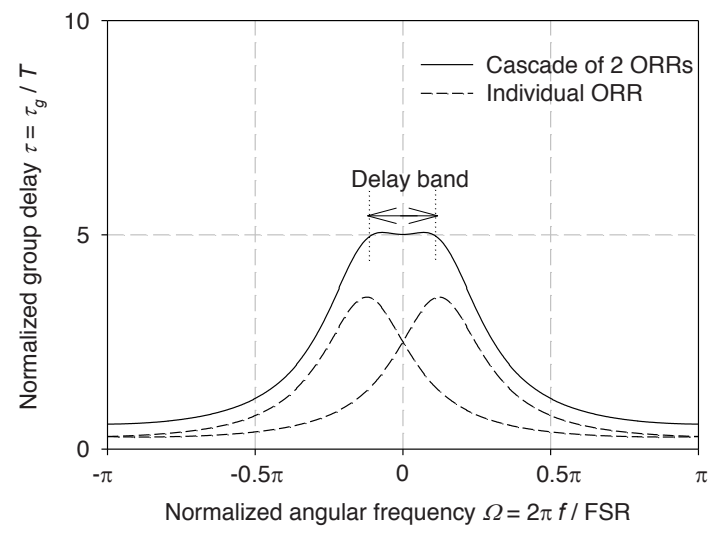

(b)

Figure 2.21: Illustration of squeezing method to reduce delay ripple of a cascade of 2 ORRs: (a) before squeezing; (b) after squeezing. 


\section{Power transmission and loss}

Although not illustrated, the power transmission response and group delay response of multi-stage cascaded ORRs still resemble the inverted version of each other, just like in the single ring resonator case. This is because both of the loss in $\mathrm{dB}$ and the group delay are generated by adding up the corresponding responses of the individual stages. The loss (attenuation) of multiple cascaded ORRs can be calculated by using Eq. (2.39).

\section{Bandwidth-delay relation}

For multiple cascaded ORRs, there are mutual tradeoffs between $\bar{\tau}, \Delta \tau, \Delta \Omega_{\mathrm{BW}}$ and the number of stages $N$. The simulation results of these tradeoffs are illustrated in Figure 2.22 and 2.23 for up to a cascade of four ORRs. In Figure 2.22 it appears that the bandwidth increase from 2 to 3 ORRs is larger than that from 1 to 2 ORRs. Compared to a cascade of 2 ORRs, a cascade of 3 ORRs has the individual group delay response of the third ORR in the middle of the delay band, which has relatively lower peak delay value than the other two at the side in order to achieve a flattened delay band (see Figure 2.20), therefore it provides more bandwidth contribution than the other two according to the tradeoff between the bandwidth and the peak delay of ORRs. For the cascade of more ORRs, one can use the approximation that the delay bandwidth increases proportionally to the number of ORRs. This is because the added ORRs will have more or less the same peak delay as the other ORRs that forms the middle part of the flattened delay band, and therefore provide roughly equal bandwidth contributions.

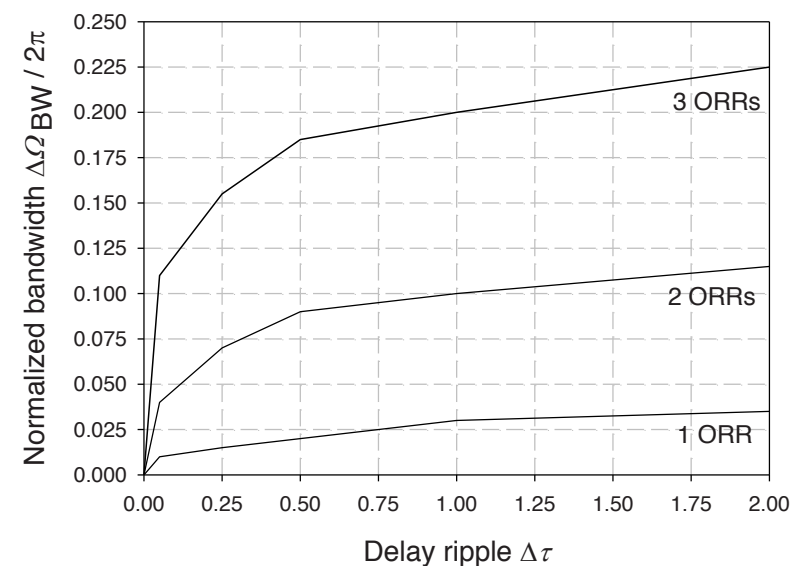

Figure 2.22: Relation between $\Delta \Omega_{\mathrm{BW}}$ and $\Delta \tau$ for different number of ORRs (the average delay value of the delay band is fixed at $\bar{\tau}=5$ ) 


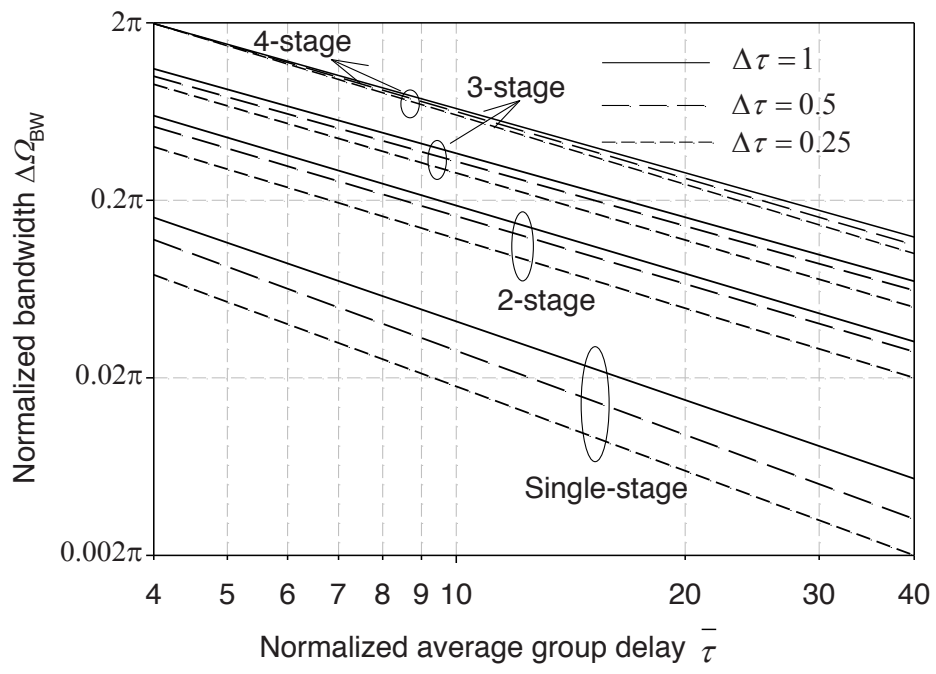

Figure 2.23: Relation between $\Delta \Omega_{\mathrm{BW}}$ and $\bar{\tau}$ for different number of stages and different $\Delta \tau$

By observing the simulation results in Figure 2.23, one can obtain the approximation that for fixed values of $\Delta \tau$ and $N, \bar{\tau}$ and $\Delta \Omega_{\mathrm{BW}}$ have a straight-line-shaped relation on a log-log scale. Based on the explanation in Section 2.4.1, the area under the group delay curve represents the total phase shift of the cascaded ORRs over one FSR, and therefore equals to the constant $2 \pi \cdot N$. Therefore, for a given pair of $\Delta \tau$ and $N$ the value of the product $\left.\bar{\tau} \cdot \Delta \Omega_{\mathrm{BW}}\right|_{\Delta \tau, N}$ can be given by

$$
\mathrm{C}_{\Delta \tau, N}=\left.\bar{\tau} \cdot \Delta \Omega_{\mathrm{BW}}\right|_{\Delta \tau, N}=\left.2 \pi \cdot \bar{\tau}_{g} \cdot \Delta f_{\mathrm{BW}}\right|_{\Delta \tau, N}=2 \pi \cdot N \cdot \mathrm{Q}(\Delta \tau)
$$

where $\varrho(\Delta \tau)=(0, \ldots, 1)$ is a factor, which indicates the effect of the delay ripple on the area under the delay band (for a fixed delay value the bandwidth of the ORRs increases/decreases with the delay ripple, as illustrated in Figure 2.22). It will be explained in Chapter 4 and 5 that the delay ripple requirement of the beam former demonstrator is $\Delta \tau \approx 0.5$, which is considered to be a condition for designing of the ORR-based delay elements (delay lines) of the beam former demonstrator. On this base, one can obtain from the simulation results in Figure 2.23 the relation:

$$
\dot{\mathrm{C}}_{N}=\mathrm{C}_{N} / 2 \pi=\left.\frac{1}{2 \pi} \cdot \bar{\tau} \cdot \Delta \Omega_{\mathrm{BW}}\right|_{N} \approx N-1
$$

Then, by combining Eq. (2.42) and Eq. (2.43), one can reach an approximation

$$
N=\operatorname{Round}\left(\dot{\mathrm{C}}_{N}\right)+1=\operatorname{Round}\left(\left.\bar{\tau}_{g} \cdot \Delta f_{\mathrm{BW}}\right|_{N}\right)+1
$$


where Round(...) is the operator of rounding off to the higher integer (to guarantee the number of ORRs is sufficient to provide the required bandwidth and delay value). Eq. (2.44) is useful for the architecture design of the ORR-based OBFN of the beam former demonstrator. One can use this equation to calculate how many ORRs are needed in a given OBFN architecture to be able to satisfy the corresponding requirements on the delay value and delay bandwidth. The architecture design of the ORR-based OBFN is further explained in Chapter 3.

\subsection{Conclusions}

With an ORR it is possible to generate higher group delays than its unit delay, which is continuously tunable. The delay of a single ORR has limited bandwidth. However, delay bandwidth enhancement can be obtained by using a cascade of multiple ORRs. Mutual tradeoffs between $\bar{\tau}, \Delta \tau, \Delta \Omega_{\mathrm{BW}}$ and the number of cascaded rings $N$ exist in the ORR-based delay elements (delay lines). In reality an ORR introduces loss, and the total loss of the ORR-based delay element increases with the delay value. Moreover, the delay functionality of ORRs can be considered to be independent of the physical dimensions of the ORRs. As a result, a formula is derived for the estimation of the necessary number of ORRs for a desired group delay response.

\section{References}

[1] W. C. van Etten, J. van der Plaats, Fundamentals of optical Fiber Communications, Prentice-Hall, Inc., 1991.

[2] J. Proakis and D. Manolakis, Digital Signal Processing: Principles, Algorithms, and Applications, $3^{\text {rd }}$. Ed., Upper Saddle River, NJ: Prentice-Hall, 1996.

[3] R. Bracewell, The Fourier Transform and Its Applications, New York: McGraw-Hill, 1986.

[4] G. Lenz, B. J. Eggleton, C. K. Madsen, and R. E. Slusher, "Optical Delay Lines Based on Optical Filters," IEEE J. Quatum Electronics, vol 37, no. 4, pp. 525532, Apr. 2001.

[5] C. K. Madsen and J. H. Zhao, Optical Filter Design and Analysis. New York: Wiley, 1999.

[6] H. P. Uranus, L. Zhuang, C. G. H. Roeloffzen, H. J. W. M. Hoekstra, "Pulse advancement and delay in an integrated-optical two-port ring-resonator circuit: direct experimental observations," Optics Letters, Vol. 32, Issue 17, pp. 26202622, August 2007.

[7] H. P. Uranus, L. Zhuang, C. G. H. Roeloffzen, H. J. W. M. Hoekstra, "Direct Experimental Observation of Pulse Temporal Behavior in Integrated-Optical Ring-Resonator with Negative Group Velocity," Proceedings of the 13th 
European Conference on Integrated Optics (ECIO'2007), Copenhagen, Denmark, 25-27 April 2007, post-deadline paper.

[8] Constantine A. Balanis, Mordern antenna handbook, John Wiley \& Sons, Inc, 2008.

[9] C. G. H. Roeloffzen, L. Zhuang, R. G. Heideman, A. Borreman, W. van Etten, "Ring resonator-based tunable optical delay line in LPCVD waveguide technology," Proc. of the $9^{\text {th }}$ IEEE/LEOS Benelux Symp., Mons, Belgium, December 2005, pp. 79-82. 


\section{Chapter 3}

\section{Ring resonator-based}

\section{optical beam forming network}

\subsection{Introduction}

An optical ring resonator-based optical beam forming network (OBFN) for a receiving phased array antenna (PAA) consists of ORR-based delay elements (delay lines) and a signal combining circuitry. Through the OBFN, the antenna element (AE) signals are synchronized and combined to achieve the desired signal. There are several combining structures which can be used for an OBFN. As mentioned in the previous chapter, the system complexity is directly related to the total number of ORRs in the OBFN. Therefore, the structure which minimizes the required number of ORRs is preferred. However, the number of ORRs is not the only criterion for OBFN design. Some other aspects of the system should also be taken into account, such as scalability and system cost. In this chapter, several possible structures for an OBFN are presented. First of all, OBFN structures for one-dimensional linear array antennas are described. Next, the concept is extended to two-dimensional PAAs. In addition to that, OBFNs involving switching matrices or multiple wavelengths will also be discussed.

\subsection{OBFN for linear array antennas}

In this section we consider only the OBFNs for single-wavelength schemes where all modulated signals in the OBFN use the same optical carrier. In this case the power of the light source is divided over the OBFN channels, and coherent optical combining needs to be performed to achieve the maximally enhanced output of the desired radio frequency (RF) signals [1]. In this scheme the optical output power is equal to the input power (assuming a lossless OBFN), and only one optical detector is required to convert the combined signals from the optical domain back to the electrical domain. Comparatively, if the signal combining is performed in the electrical domain, the criteria for coherent combining are relaxed, however, each OBFN channel needs a separate optical detector, which increases the system complexity. 


\subsubsection{Parallel-structured OBFNs}

Based on this single-wavelength scheme, the most straightforward way to construct an OBFN is to use an architecture with two stages: a parallel-structured delay stage followed by a combining stage (in short we call it the parallel structure). With the parallel structure each OBFN channel uses an independent ORR-based delay element (DE). Those DEs can be arranged in both symmetrical and asymmetrical manner in the OBFN.

\section{Symmetrical parallel structure}

A schematic layout of an OBFN with symmetrical parallel structure is given in Figure 3.1. In this example the OBFN has four inputs corresponding to a 4-element linear array antenna. The beam angle is defined as the angle between antenna normal and beam direction. The positive and negative sign of the beam angle indicates whether $\mathrm{AE} 1$ or $\mathrm{AE} 4$ is the last $\mathrm{AE}$ to receive a wavefront. If the signal from this last $\mathrm{AE}$ is considered as the reference for signal synchronization, the signals from other AEs will then require advanced-time offsets compared to the reference signal. The further an $\mathrm{AE}$ is spaced from the reference $\mathrm{AE}$, the larger the time offset of the corresponding signal will be, and therefore the larger delay is required in the corresponding OBFN channel to compensate this time offset.

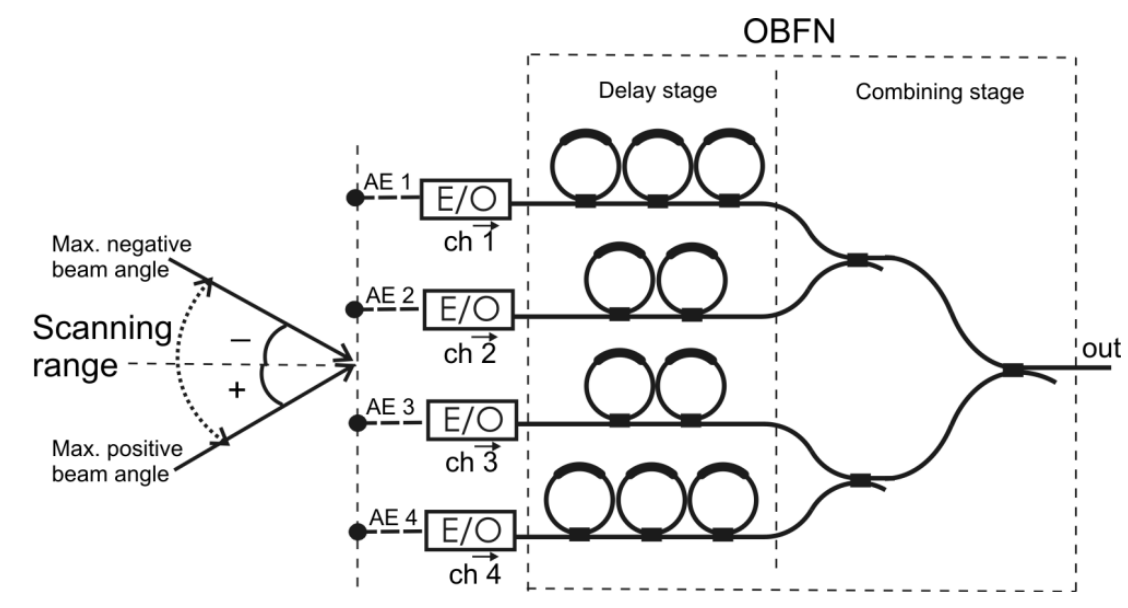

Figure 3.1: Schematic layout of a $4 \times 1$ OBFN with symmetric parallel structure

For the positive beam angle, $\mathrm{AE} 1$ is the reference $\mathrm{AE}$, and the signals from $\mathrm{AE} 2,3$, and 4 have linearly increasing time offsets. For the negative beam angle, AE 4 is the

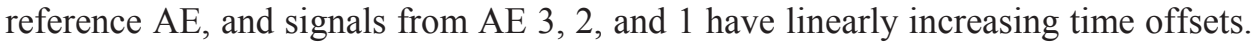


If the antenna is desired to have a beam scanning range $\Delta \theta$, then we define $\theta_{\max }^{\mathrm{p}}=\Delta \theta / 2$ and $\theta_{\max }^{\mathrm{n}}=-\Delta \theta / 2$ as the maximum positive and negative beam angle, respectively. Correspondingly, an OBFN with symmetrical parallel structure is needed. As shown in Figure 3.1, channel 1 has the same number of ORRs as channel 4; channel 2 has the same number of ORRs as channel 3. This structure is able to provide linearly increasing delays from channel 1 to channel 4 for positive beam angles and also from channel 4 to channel 1 for negative beam angles. Here, we consider that the delay differences between the signal paths of the beam former are only caused by the DEs in the OBFN. In practice when all the DEs in the OBFN are tuned to their minimum delays, still some delay differences would exist between the OBFN channels due to slight channel length differences from the OBFN mask design. However, this can be compensated by adjusting the lengths of the RF paths.

When the beam scanning range, number of antenna elements, interelement distance, and signal bandwidth are specified, one can estimate how many ORRs are necessary to use in the corresponding OBFN. For a general solution, we consider now an $M$ channel OBFN for an $M$-element linear array antenna ( $M$ is an even number). The total number of ORRs in the OBFN $N_{\text {TT }}$ is calculated by

$$
N_{\mathrm{TT}}=\sum_{m=1}^{M} N_{m}
$$

where $N_{m}$ represents the number of ORRs in channel $m$ and can be calculated with the following steps. First, we calculate the maximum time offsets of AE $m: M_{-} T_{m}^{A E}$. The calculation is based on the beam scanning range $\Delta \theta:\left[\theta_{\max }^{\mathrm{n}}=-\Delta \theta / 2\right.$, $\left.\theta_{\max }^{\mathrm{p}}=\Delta \theta / 2\right]$, which is expressed by

$$
\begin{aligned}
& M_{-} T_{m}^{A E} \\
& \quad= \begin{cases}-\frac{\sin \left(\left|\theta_{\max }^{\mathrm{p}}\right|\right) \cdot(m-1) d}{c_{0}}=-\frac{\sin \left(\frac{\Delta \theta}{2}\right) \cdot(m-1) d}{c_{0}} & \left(\frac{M}{2}+1 \leq m \leq M\right) \\
-\frac{\sin \left(\left|\theta_{\max }^{\mathrm{n}}\right|\right) \cdot(M-m) d}{c_{0}}=-\frac{\sin \left(\frac{\Delta \theta}{2}\right) \cdot(M-m) d}{c_{0}} & \left(1 \leq m \leq \frac{M}{2}\right)\end{cases}
\end{aligned}
$$

where $d$ is the interelement distance of the antenna, $c_{0}$ is the speed of light in vacuum, and the negative sign in front of the fraction indicates that the AE signal has advanced-time offset compared to the reference signal. If the signals have a bandwidth of $\Delta f_{s}$, then the corresponding group delay response of the ORR-based delay element (DE) of channel $m$ is characterized by $\Delta f_{\mathrm{BW}}=\Delta f_{s}$ and $\bar{\tau}_{g}=-M_{-} T_{m}^{A E}$. In addition to that, delay ripple $\Delta \tau=0.5$ is used as a characteristic 
of the ORR-based DEs as explained in Chapter 2. Then, Eq. (2.44) can be used here for the calculation of $N_{m}$, which is given by

$$
N_{m}=\left[\operatorname{Round}\left(\bar{\tau}_{g} \cdot \Delta f_{\mathrm{BW}}\right)+1\right]_{m}=\left[\operatorname{Round}\left(-M_{-} T_{m}^{A E} \cdot \Delta f_{s}\right)+1\right]
$$

Then, by inserting Eq.(3.3) in Eq.(3.1) and considering the symmetry of the OBFN, the total number of ORRs in the OBFN is given by

$$
\begin{aligned}
N_{\mathrm{TT}}\left(M, d, \Delta \theta, \Delta f_{s}\right) & =\sum_{m=1}^{M} N_{m}=2 \sum_{m=1}^{M / 2} N_{m} \\
& \left.=2 \sum_{m=1}^{M / 2}\left[\frac{\sin (\Delta \theta / 2) \cdot(M-m) d}{C_{0}} \cdot \Delta f_{s}\right)+1\right]
\end{aligned}
$$

A numerical example corresponding to Eq. (3.4) is given in Example 3.1.

\section{Example 3.1}

Antenna specifications: $M=4 ; d=25 \mathrm{~cm} ; \Delta \theta=60^{\circ} ; \Delta f_{s}=1 \mathrm{GHz}$

$N_{1}=N_{4}=\operatorname{Round}\left(M_{-} T_{1}^{A E} \cdot \Delta f_{S}\right)+1$

$=\operatorname{Round}\left(\frac{\sin \left(30^{\circ}\right) \times(4-1) \times 25 \times 10^{-2}}{3 \times 10^{8}} \cdot 1 \times 10^{9}\right)+1=2+1=3$

$N_{2}=N_{3}=\operatorname{Round}\left(M_{-} T_{2}^{A E} \cdot \Delta f_{s}\right)+1$

$=\operatorname{Round}\left(\frac{\sin \left(30^{\circ}\right) \times(4-2) \times 25 \times 10^{-2}}{3 \times 10^{8}} \cdot 1 \times 10^{9}\right)+1=1+1=2$

$N_{\mathrm{TT}}=2 \sum_{m=1}^{M / 2} N_{m}=2\left(N_{1}+N_{2}\right)=10$

\section{Asymmetrical parallel structure}

The schematic layout of a 4-channel OBFN with asymmetrical parallel structure is given in Figure 3.2. Unlike the OBFN with symmetrical parallel structure, this OBFN always uses AE 1 as the reference (or AE 4 if the OBFN is turned upside

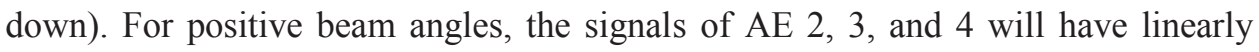
increasing advanced-time offsets compared to the reference signal of AE 1. These time offsets can be compensated by the delays provided by the ORRs in the OBFN. However, for the negative beam angle, the signals of $\mathrm{AE} 2,3$, and 4 have linearly increasing delayed-time offsets compared to the reference signal of $\mathrm{AE} \mathrm{1,} \mathrm{which}$ cannot be compensated by ORRs. To solve this, fixed delays can be added to the signals before they enter the ORRs as shown in Figure 3.2. The added delays should be such that they compensate the delayed-time offsets for the maximum negative beam angle. Then the AE signals will have advanced-time offsets for all the beam angels within the scanning range, which are two time as long as those when the system has no additional fixed delay as the example shown in Figure 3.1. Therefore, 
when calculating the antenna time offset, a factor of 2 needs to be added, compared to Eq. (3.2). This will be seen in Eq. (3.5). The additional fixed delays can be achieved by introducing additional signal path length differences either in the RF paths or in the optical paths. The example in Figure 3.2 shows the additional signal path length differences in the RF paths.

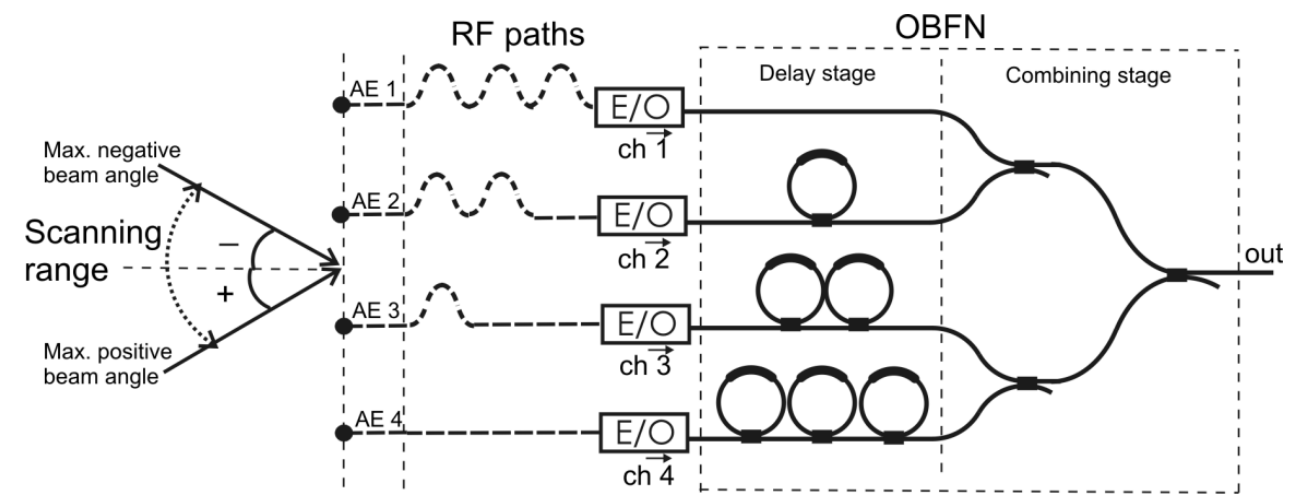

Figure 3.2: Schematic layout of a $4 \times 1$ OBFN with asymmetrical parallel structure

Consider now a general $M$-channel OBFN with an asymmetrical parallel structure and additional fixed delays. Channel 1 is used as the reference channel. The beam scanning range and the interelement distance of the antenna are $\Delta \theta$ and $d$, respectively. Then, the maximum time offset of AE $m$ can be calculated by

$$
M_{-} T_{m}=-\frac{2 \sin \left(\frac{\Delta \theta}{2}\right) \cdot(m-1) d}{c_{0}}
$$

Correspondingly, $N_{m}$ and $N_{\text {TT }}$ of the OBFN can be calculated by

$$
\begin{aligned}
& N_{m}=\left[\operatorname{Round}\left(\bar{\tau}_{g} \cdot \Delta f_{\mathrm{BW}}\right)+1\right]_{m}=\left[\operatorname{Round}\left(-M_{-} T_{m} \cdot \Delta f_{s}\right)+1\right] \\
& N_{\mathrm{TT}}\left(M, d, \Delta \theta, \Delta f_{s}\right)=\sum_{m=1}^{M} N_{m} \\
&=\sum_{m=1}^{M}\left[\operatorname{Round}\left(\frac{2 \sin \left(\frac{\Delta \theta}{2}\right) \cdot(m-1) d}{c_{0}} \cdot \Delta f_{s}\right)+1\right]
\end{aligned}
$$

A numerical example corresponding to Eq. (3.7) is given in Example 3.2.

\section{Example 3.2}

Antenna specifications: $M=4 ; d=25 \mathrm{~cm} ; \Delta \theta=60^{\circ}$

Signal bandwidth: $\Delta f_{s}=1 \mathrm{GHz}$ 


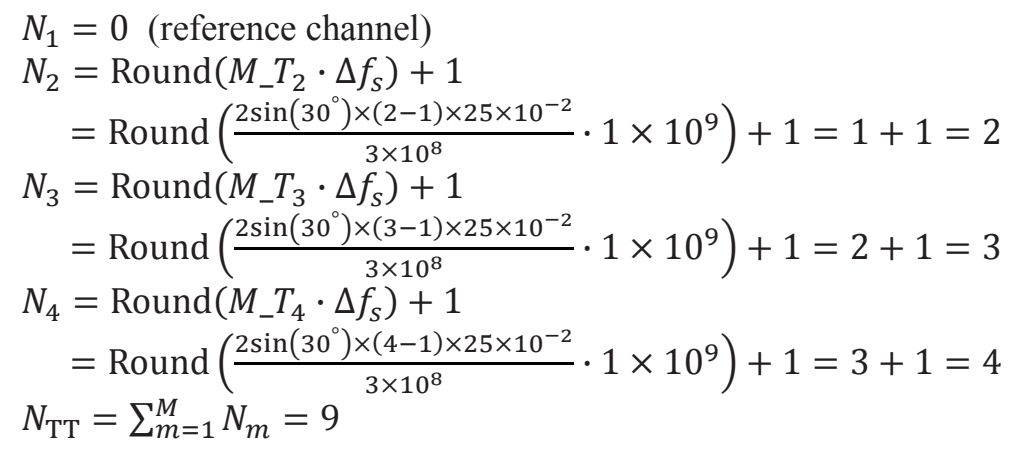

\subsubsection{Binary-tree-structured OBFNs}

As shown in the previous section, one way to construct an OBFN is to connect a delay stage to a separate combining stage. Instead of this, one can also construct an OBFN with multiple binary-combining stages, where signals of each two neighboring channels are synchronized and combined separately. The corresponding OBFN structure is called binary-tree structure.

Unlike the parallel structure, the binary-tree structure divides the signal combining process into multiple steps. First of all, the entire AE array is divided into multiple AE pairs formed by two neighboring AEs, and the signals of the each AE pair are synchronized and combined. After the first step, the two adjacent AE signals of each AE pair can be considered to have merged into one, and therefore a new imaginary $\mathrm{AE}$ array is then formed with half the number of elements and twice interelement distance. Then in the second step, this new imaginary AE array is again divided into multiple AE pairs, and each AE pair is synchronized and combined separately. This process will continue in such steps until all the AE signals are combined.

Each combining step requires a binary-combining stage consisting of ORR-based DEs and combining circuitry. The number of ORRs in each DE depends on the delay requirement of the corresponding combining step. When all the stages are connected together, a binary-tree structured OBFN is obtained. For a binary-tree structured OBFN, the number of inputs $M$ is given by $M=2^{K}$ ( $K$ is the number of stages), and the ORR-based delay elements can be arranged in both symmetrical and asymmetrical manner between the channels.

\section{Symmetrical binary-tree structure}

A schematic layout of a 4-channel OBFN with symmetrical binary-tree structure is given in Figure 3.3. 


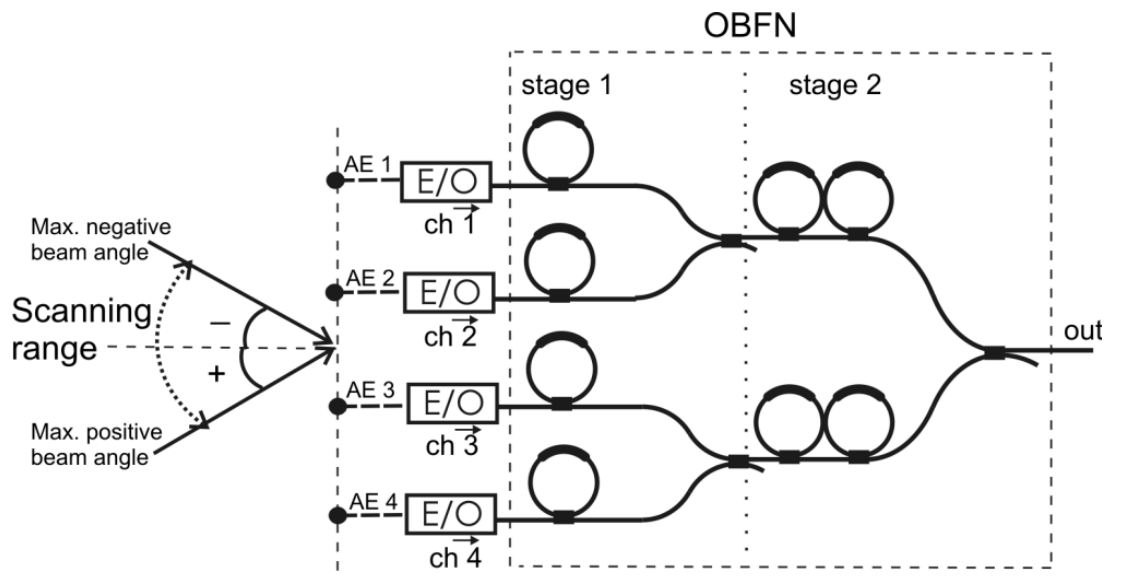

Figure 3.3: Schematic layout of a symmetrical-binary-tree-structured $4 \times 1$ OBFN

In this figure, the OBFN is connected to a 4-element linear array antenna. To combine the signals from the four AEs, the OBFN consists of two stages. In each stage, both the upper and lower arms of a binary-combining section are equipped with a DE. This is because of the existence of both positive and negative beam angles. For a general $M=2^{K}$-element linear array antenna, the distance between two neighboring AEs in each combining step is given by $2^{k-1} \cdot d$ where $k$ is the stage number $k=[1, \ldots, K], d$ is the interelement distance), and since the beam scanning range is defined symmetrical about the normal of the antenna plane: $\left[\theta_{\max }^{\mathrm{n}}=-\Delta \theta / 2, \theta_{\max }^{\mathrm{p}}=\Delta \theta / 2\right]$, the AE signals for the upper and lower arms of each binary-combining section have equal maximum time offset. For positive beam angles, the signal for the lower arm has advanced-time offset against that for the upper arm; for the negative beam angles, it is the other way around. The maximum time offset of $k$ th stage $M_{-} T_{k}$ can be calculated by

$$
M \_T_{k}=-\frac{\sin (\Delta \theta / 2) \cdot 2^{k-1} \cdot d}{c_{0}}
$$

where the negative sign indicates that it is an advanced-time offset. For an $M$ channel binary-tree structured OBFN, the total number of ORRs in the OBFN $N_{\mathrm{TT}}$ is calculated by adding up the number of ORRs of all binary-combining sections:

$$
N_{\mathrm{TT}}=\sum_{k=1}^{K} \frac{M}{2^{k}} \cdot N_{k}
$$

where $M / 2^{k}$ is the number of binary-combining sections in OBFN stage $k$, and $N_{k}$ is the number of ORRs in a binary-combining section of stage $k$. Then, using Eq. (2.44) the value of $N_{k}$ can be calculated by 


$$
\begin{aligned}
N_{k} & =N_{k}^{U}+N_{k}^{L}=2 N_{k}^{U}=2\left[\operatorname{Round}\left(\bar{\tau}_{g} \cdot \Delta f_{\mathrm{BW}}\right)+1\right]_{k} \\
& =2\left[\operatorname{Round}\left(-M T_{k} \cdot \Delta f_{s}\right)+1\right]
\end{aligned}
$$

where $N_{k}^{U}$ and $N_{k}^{L}$ represents the number of ORRs of the upper and lower arm of the binary-combining section, which are equal because of the structural symmetry. Further, by combining Eq. (3.8) - Eq. (3.10) the total number of ORRs in the OBFN in given by

$$
\begin{aligned}
N_{\mathrm{TT}}\left(M, d, \Delta \theta, \Delta f_{S}\right) & =\sum_{k=1}^{K} \frac{M}{2^{k}} \cdot N_{k} \\
& =\sum_{k=1}^{K} \frac{M}{2^{k}} \cdot 2\left[\operatorname{Round}\left(\frac{\sin (\Delta \theta / 2) \cdot 2^{k-1} \cdot d}{c_{0}} \cdot \Delta f_{S}\right)+1\right](
\end{aligned}
$$

A numerical example corresponding to Eq. (3.11) is given in Example 3.3.

\section{Example 3.3}

Antenna specifications: $M=2^{K}=4(K=2) ; d=25 \mathrm{~cm} ; \Delta \theta=60^{\circ}$

Signal bandwidth: $\Delta f_{s}=1 \mathrm{GHz}$

$$
\begin{aligned}
& N_{1}=2 \times\left[\operatorname{Round}\left(\frac{\sin \left(30^{\circ}\right) \times 1 \times 25 \times 10^{-2}}{3 \times 10^{8}} \cdot 1 \times 10^{9}\right)+1\right]=2 \times[1+1]=4 \\
& N_{2}=2 \times\left[\text { Round }\left(\frac{\sin \left(30^{\circ}\right) \times 2 \times 25 \times 10^{-2}}{3 \times 10^{8}} \cdot 1 \times 10^{9}\right)+1\right]=2 \times[1+1]=4 \\
& N_{\mathrm{TT}}=\sum_{k=1}^{K} \frac{M}{2^{k}} \cdot N_{k}=2 \times 4+1 \times 4=12
\end{aligned}
$$

\section{Asymmetrical binary-tree structure}

The schematic layout of a 4-channel OBFN with asymmetrical binary-tree structure is given in Figure 3.4. In this scheme, channel 1 is used as the reference channel for all beam angles. Like the scheme for the OBFN with asymmetrical parallel structure, the fixed delays are added in the RF paths to compensate the delayed-time offsets caused by the negative beam angles. This way, for all beam angles in the scanning range the signal for the lower arm of each binary-combining section has advancedtime offset against that for the upper arm. In this case only the lower arms of the binary-combining sections need DEs.

For the $k$ th stage of the OBFN, the maximum time offset of the AE signal for the lower arm of a binary-combining section occurs when the antenna beam is at the maximum positive angle. Using the same time-offset principle as for the OBFN with 


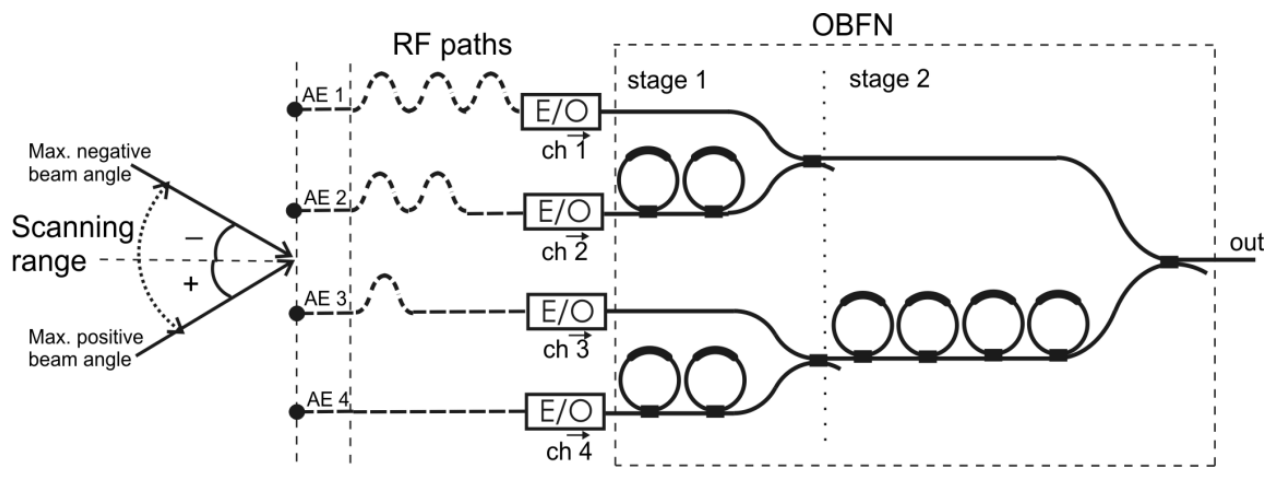

Figure 3.4:Schematic layout of an asymmetrical-binary-tree-structured $4 \times 1$ OBFN

asymmetrical parallel structure, this maximum advanced-time offset $M_{-} T_{k}$ is two times as long as that for the OBFN with symmetric binary-tree structure. Then, $M_{-} T_{k}$ of an $M$-channel ( $M=2^{K}, K$ is the total number of stages) asymmetrical binary-tree-structured OBFN can be calculated by

$$
M_{-} T_{k}=-\frac{2 \sin (\Delta \theta / 2) \cdot 2^{k-1} \cdot d}{c_{0}}
$$

Correspondingly, $N_{k}$ and $N_{\text {TT }}$ of the OBFN can be estimated by

$$
\begin{aligned}
N_{k}=\left[\operatorname{Round}\left(\bar{\tau}_{g} \cdot \Delta f_{\mathrm{BW}}\right)+1\right]_{k}=\left[\operatorname{Round}\left(-M_{-} T_{k} \cdot \Delta f_{S}\right)+1\right] \\
N_{\mathrm{TT}}\left(M, d, \Delta \theta, \Delta f_{S}\right)=\sum_{k=1}^{K} \frac{M}{2^{k}} \cdot N_{k} \\
=\sum_{k=1}^{K} \frac{M}{2^{k}} \cdot\left[\operatorname{Round}\left(\frac{2 \sin (\Delta \theta / 2) \cdot 2^{k-1} \cdot d}{c_{0}} \cdot \Delta f_{S}\right)+1\right]
\end{aligned}
$$

A numerical example corresponding to Eq. (3.14) is given in Example 3.4.

\section{Example 3.4}

Antenna specifications: $M=2^{K}=4(K=2) ; d=25 \mathrm{~cm} ; \Delta \theta=60^{\circ}$

Signal bandwidth: $\Delta f_{S}=1 \mathrm{GHz}$

$$
\begin{aligned}
& N_{1}=\operatorname{Round}\left(\frac{2 \sin \left(30^{\circ}\right) \times 1 \times 25 \times 10^{-2}}{3 \times 10^{8}} \cdot 1 \times 10^{9}\right)+1=1+1=2 \\
& N_{2}=\operatorname{Round}\left(\frac{2 \sin \left(30^{\circ}\right) \times 2 \times 25 \times 10^{-2}}{3 \times 10^{8}} \cdot 1 \times 10^{9}\right)+1=2+1=3 \\
& N_{\mathrm{TT}}=\sum_{k=1}^{K} \frac{M}{2^{k}} \cdot N_{k}=2 \times 2+1 \times 3=7
\end{aligned}
$$


Based on the above four numerical examples, it is shown that for the same antenna specifications and signal bandwidth the OBFN with the asymmetrical binary-tree structure leads to the least number of necessary ORRs. Besides, it also benefits the scalability of the system. Each time adding one more stage in the OBFN means the number of input ports will be doubled. A corresponding OBFN with three stages is shown in Figure 3.5, which is an extension of the OBFN in Figure 3.4.

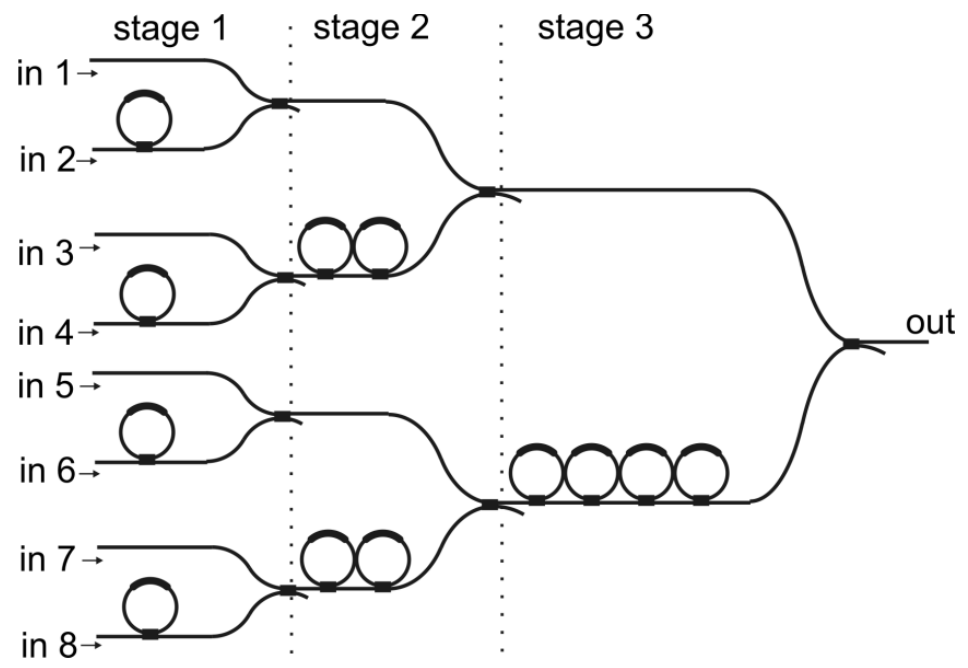

Figure 3.5: Schematic of an $8 \times 1$ OBFN with asymmetric binary-tree structure

\subsection{OBFN for rectangular $M \times N$ array antennas}

A rectangular $M \times N$ array antenna can be regarded as $M$ sets of $N$-element linear subarrays placed parallel to one another [2], as shown in Figure 3.6.

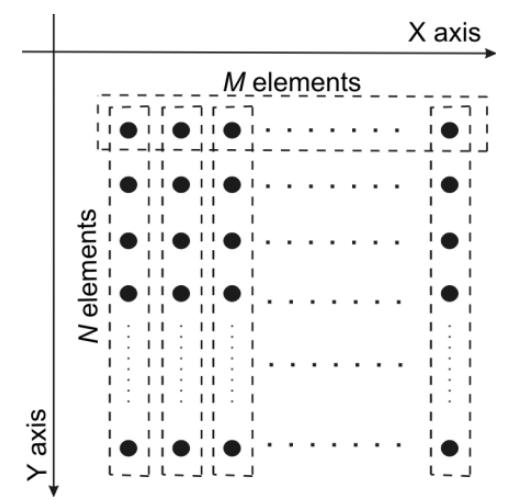

Figure 3.6: $M \times N$ array antenna 
In this case signal combining of the antenna elements can be performed analogously to that of the linear array antenna, but in two steps. In the first step the same signal combining process is performed in all $\mathrm{N}$-element linear sub-arrays (along the $\mathrm{Y}$-axis up) in a parallel manner. This step results in a new imaginary $M$-element linear array lying along the $\mathrm{X}$-axis as illustrated in Figure 3.6. Then in the second step the signal combining of this $M$-element array is performed.

The corresponding OBFN consists of two sections for the two signal combining steps. The first section has $M$ parallel sets of $N$-channel combining circuits and the second section combines the outputs of the first section by an $M$-channel combining circuit. If $M=2^{K}, N=2^{W}$ ( $K$ and $W$ are integers), and the beam scanning ranges in the $\mathrm{X}-\mathrm{Z}$ and the $\mathrm{Y}-\mathrm{Z}$ plane, $\Delta \theta_{\mathrm{XZ}}$ and $\Delta \theta_{\mathrm{YZ}}$, are both symmetrical with regard to the antenna normal, then the asymmetrical binary-tree structure can be used for those $N$ and $M$-channel combining circuits of the OBFN. This is illustrated by the schematic of the $16 \times 1$ OBFN for a $4 \times 4$ antenna array which is shown in Figure 3.7. Correspondingly, based on Eq. (3.14), the total number of necessary ORRs in the $M \times N$-channel OBFN $N_{\mathrm{TT}}^{M \times N}$ can be calculated by

$N_{\mathrm{TT}}^{M \times N}\left(M, N, d, \Delta \theta_{\mathrm{YZ}}, \Delta \theta_{\mathrm{XZ}}, \Delta f_{s}\right)=M \cdot N_{\mathrm{TT}}\left(N, d, \Delta \theta_{\mathrm{YZ}}, \Delta f_{s}\right)+N_{\mathrm{TT}}\left(M, d, \Delta \theta_{\mathrm{XZ}}, \Delta f_{s}\right)$

Alternatively, a rectangular $M \times N$ array antenna can also be regarded as $N$ sets of $M$ element linear sub-arrays placed parallel to one another. In this case the first section of the OBFN has $N$ parallel sets of $M$-channel combining circuits and the second section combines the outputs of the first section by an $N$-channel combining circuit. Then the total number of necessary ORRs in the $M \times N$-channel OBFN $N_{\mathrm{TT}}^{M \times N}$ can be calculated by

$N_{\mathrm{TT}}^{M \times N}\left(N, M, d, \Delta \theta_{\mathrm{XZ}}, \Delta \theta_{\mathrm{YZ}}, \Delta f_{s}\right)=N \cdot N_{\mathrm{TT}}\left(M, d, \Delta \theta_{\mathrm{XZ}}, \Delta f_{s}\right)+N_{\mathrm{TT}}\left(N, d, \Delta \theta_{\mathrm{YZ}}, \Delta f_{s}\right)$

The choice of the above two combining schemes for the OBFN depends on which one leads to less ORRs, namely lower OBFN complexity.

A numerical example is given in Example 3.5.

\section{Example 3.5}

Antenna specifications: $M=4 ; N=4 ; d=10 \mathrm{~cm} ; \Delta \theta_{\mathrm{XZ}}=60^{\circ} ; \Delta \theta_{\mathrm{YZ}}=60^{\circ}$;

Signal bandwidth: $\Delta f_{S}=1 \mathrm{GHz}$

$$
\begin{aligned}
& N_{\mathrm{TT}}\left(N, d, \Delta \theta_{\mathrm{YZ}}, \Delta f_{s}\right) \\
& \quad=2 \times\left[\operatorname{Round}\left(\frac{2 \sin \left(30^{\circ}\right) \times 1 \times 10 \times 10^{-2}}{3 \times 10^{8}} \cdot 10^{9}\right)+1\right]+\left[\operatorname { R o u n d } \left(\frac{2 \sin \left(30^{\circ}\right) \times 2 \times 10 \times 10^{-2}}{3 \times 10^{8}} .\right.\right.
\end{aligned}
$$



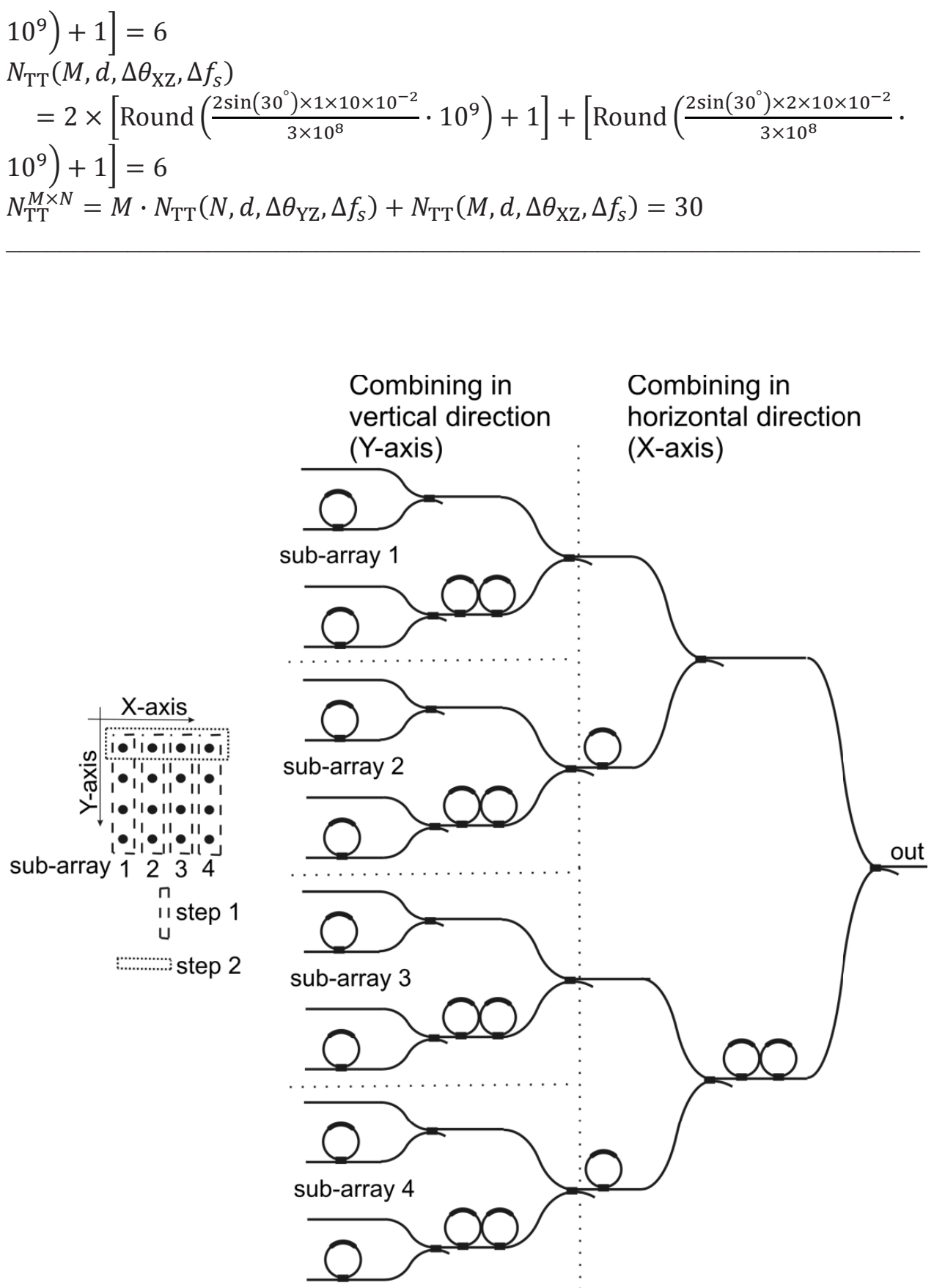

Figure 3.7: Schematic layout of a 16-channel OBFN with asymmetrical binarytree structure for a $4 \times 4$ array antenna 


\subsection{Alternative schemes for an OBFN}

As explained before, the system complexity is directly related to the number of ORRs in the OBFN. Besides the OBFN schemes described previously, some alternative OBFN schemes which can further reduce the number of ORRs are described in this section. However, these alternative OBFN schemes may also bring other practical disadvantages.

\section{Adding a switch matrix in front of the $\mathrm{OBFN}$}

For a given PAA, the antenna size and desired beam scanning range are the factors that determine the maximum delay requirement for the delay elements in the OBFN. As shown in Section 3.2, the asymmetrical binary-tree structure is more advantageous for the OBFN complexity than the other three structures, and the corresponding DEs must be able to provide delays for both positive and negative beam angles. However, if the OBFN is only needed to provide delays for positive angles $\left[0, \theta_{\max }^{\mathrm{P}}=\Delta \theta / 2\right]$, then the additional path length differences can be removed and correspondingly the maximum time offset $M_{-} T_{k}$ for the $k$ th stage of an $M$ channel $\left(M=2^{K}\right)$ asymmetrical binary-tree structured OBFN can be calculated by

$$
M_{-} T_{k}=-\frac{\sin (\Delta \theta / 2) \cdot 2^{k-1} \cdot d}{c_{0}}
$$

Then, $N_{k}$ and $N_{\mathrm{TT}}$ of the OBFN can be estimated by

$$
\begin{gathered}
N_{k}=\left[\operatorname{Round}\left(\bar{\tau}_{g} \cdot \Delta f_{\mathrm{BW}}\right)+1\right]_{k}=\left[\operatorname{Round}\left(-M_{-} T_{k} \cdot \Delta f_{S}\right)+1\right] \\
N_{\mathrm{TT}}\left(M, d, \Delta \theta, \Delta f_{S}\right)=\sum_{k=1}^{K} \frac{M}{2^{k}} \cdot\left[\operatorname{Round}\left(\frac{\sin (\Delta \theta / 2) \cdot 2^{k-1} \cdot d}{c_{0}} \cdot \Delta f_{S}\right)+1\right]
\end{gathered}
$$

Thus, the total number of necessary ORRs will decrease resulting in a lower OBFN complexity. Then, the question is how to achieve the negative beam angles using this OBFN. The solution is to use a switch matrix between the AEs and the OBFN inputs. This switch matrix can be implemented in the electrical or in the optical domain [3], [4]; it should be able to work in two different states: bar state (AE $n$ to $\mathrm{CH} n$ ) and cross state (AE $n$ to $\mathrm{CH} N-n+1$ ), as shown in Figure 3.8. Assume that all the signal paths in the switch matrix have equal path lengths, and the DEs of the OBFN are set to achieve a positive beam angle $\theta$ with the switch matrix in bar state. Then, when the switch matrix changes to cross state, the beam will be flipped to its mirror-image position with respect to the antenna normal, resulting in the negative beam angle $-\theta$. This way, the system is capable of reaching both positive and negative beam angles. 


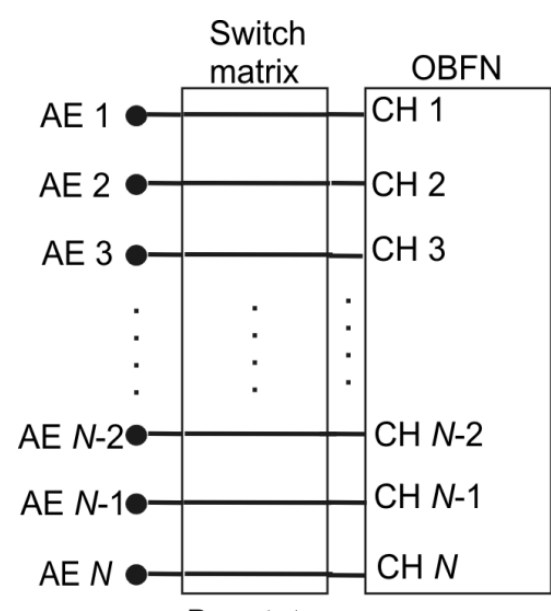

Bar state

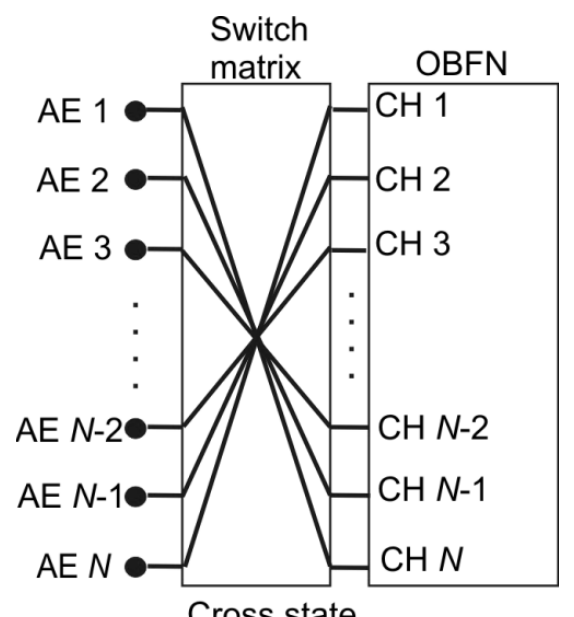

Cross state

Figure 3.8: Bar state and cross state of the switch matrix

This idea can also be applied to the OBFN for $M \times N$ array antennas. As illustrated in Figure 3.7, an OBFN for array antennas can be regarded as a combination of multiple sub-OBFNs for linear array antennas, thus a switch matrix can be added to each of the sub-OBFNs. A numerical example which compares OBFNs with and without a switch with respect to the total number of necessary ORRs is given in Example 3.6. The corresponding OBFNs are shown in Figure 3.9. Compared to the non-switched case, the switch scheme can approximately reduce the total number of necessary ORRs by a factor of two, resulting in a lower OBFN complexity. But the disadvantage of this scheme is that it may eventually increase the complexity of the entire beam former system due to the additional switching circuit. The complexity of the switching circuit and its control system increases fast with its number of inputs, especially when the switching is performed in the optical domain [3], [4].

\section{Example 3.6}

Antenna specifications: $M=2(K=1) ; d=40 \mathrm{~cm} ; \Delta \theta=60^{\circ}\left(\theta_{\max }^{\mathrm{P}}=\Delta \theta / 2=\right.$ $30^{\circ}$ ); Signal bandwidth: $\Delta f_{s}=2 \mathrm{GHz}$; OBFN structure: asymmetrical binary-tree;

Without switch: $N_{\mathrm{TT}}=\sum_{k=1}^{K} \frac{M}{2^{k}} \cdot\left[\operatorname{Round}\left(\frac{2 \sin \left(\frac{\Delta \theta}{2}\right) \cdot k \cdot d}{c_{0}} \cdot \Delta f_{s}\right)+1\right]$

$$
=\operatorname{Round}\left(\frac{2 \sin \left(30^{\circ}\right) \times 40 \times 10^{-2}}{3 \times 10^{8}} \cdot 2 \times 10^{9}\right)+1=4
$$

With switch: $N_{\mathrm{TT}}=\sum_{k=1}^{K} \frac{M}{2^{k}} \cdot\left[\operatorname{Round}\left(\frac{\sin \left(\frac{\Delta \theta}{2}\right) \cdot k \cdot d}{c_{0}} \cdot \Delta f_{S}\right)+1\right]$

$$
=\operatorname{Round}\left(\frac{\sin \left(30^{\circ}\right) \times 40 \times 10^{-2}}{3 \times 10^{8}} \cdot 2 \times 10^{9}\right)+1=2
$$



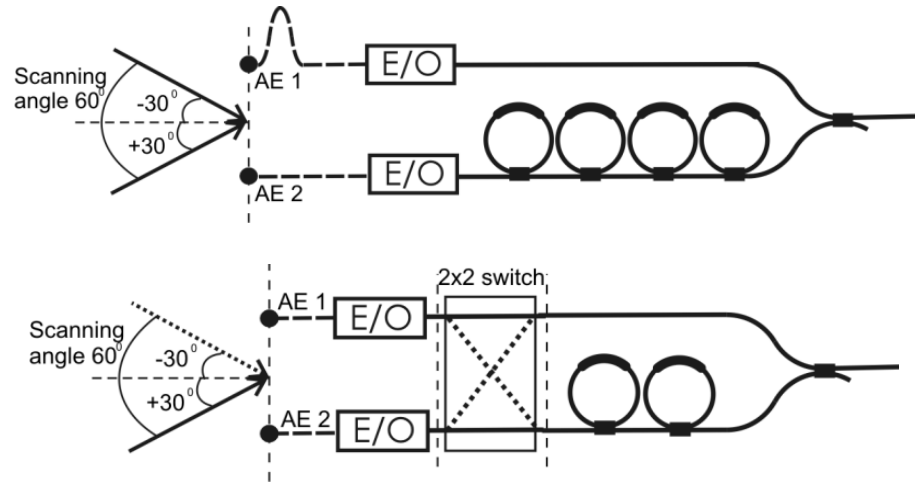

Figure 3.9: OBFNs without (upper) and with switch (lower) for the same beam scanning angle

\section{Multi-wavelength scheme}

Instead of a single-wavelength OBFN system, one can also design an multiwavelength OBFN which makes use of the wavelength periodicity of the ORRs. To simplify the explanation, we use an OBFN for a 4-element linear array antenna for instance; four optical carriers with different wavelengths are assigned to four AEs, as shown in Figure 3.10.

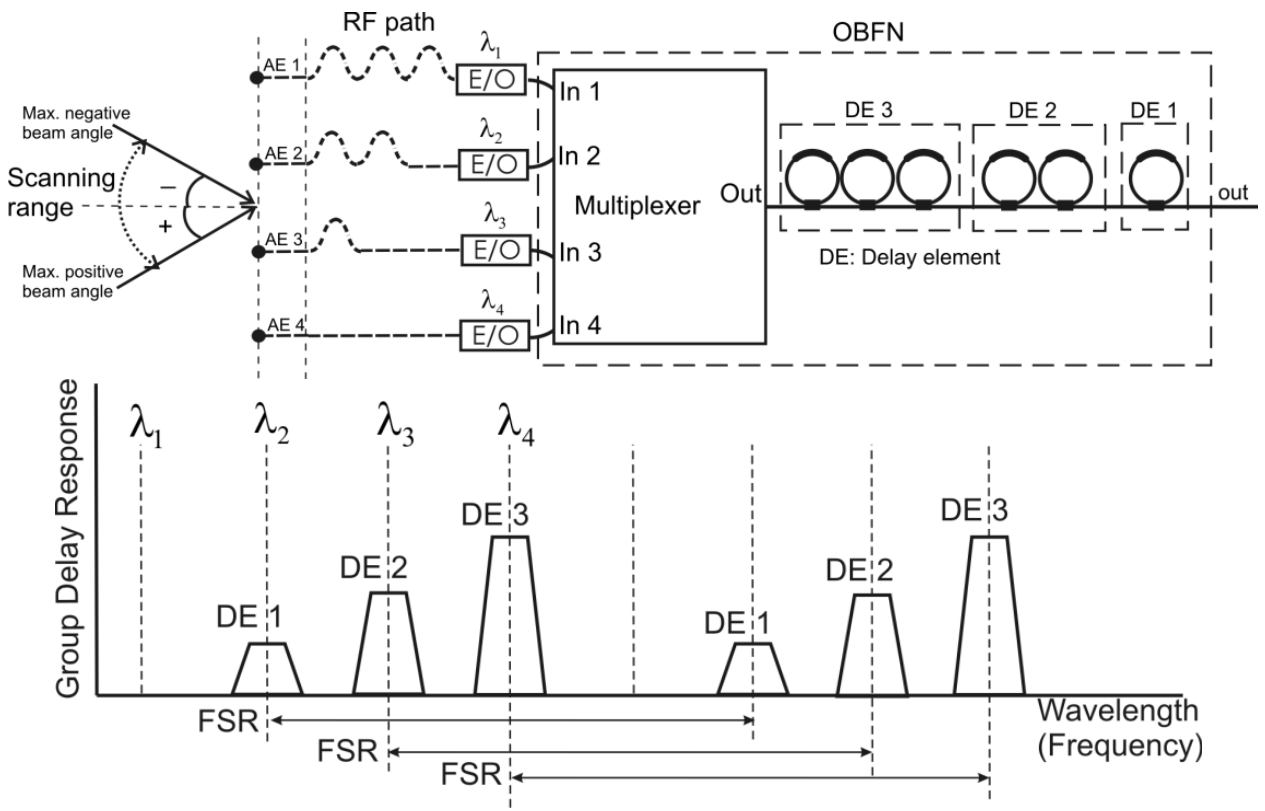

Figure 3.10: Drawings of a 4-channel multi-wavelength OBFN with equal-FSR delay elements (upper) and its group delay response (lower) 
If the signal of $\mathrm{AE} 1$ (on $\lambda_{1}$ ) is defined as the reference for signal synchronization, then the OBFN needs only to compensate the time offsets of the other three AE signals (on $\lambda_{2}, \lambda_{3}$, and $\lambda_{4}$ ). As explained in Section 3.2, when AE 1 is the reference $\mathrm{AE}$ for both positive and negative beam angles, the straightforward way to construct the OBFN is to use the asymmetrical parallel structure as shown in Figure 3.2. However, in this multi-wavelength case, the ORR-based DEs are tuned to generate their bandwidth-limited delay bands at $\lambda_{2}, \lambda_{3}$, and $\lambda_{4}$, respectively, instead of at the same wavelength. This way, the delay bands of those DEs do not overlap in the optical frequency (wavelength) domain provided that the frequency intervals between the optical carriers are larger than the delay bandwidth. Owing to this delay-band separation and the fact that signals on different wavelengths do not interfere with one another, those parallel delay paths in the OBFN can be replaced by a common path with all the DEs placed in a cascade as shown in Figure 3.10. To guide the four AE signals into a common signal path, an optical multiplexer can be used for almost lossless combining [5], [6]. Instead of using a multiplexer, a simple $2 \times 2$ coupler-based binary-tree combining structure can also be used to combine multiple wavelengths, however, this will result in an additional $3 \mathrm{~dB}$ optical power loss at each coupler [7]. Moreover, the DEs in the OBFN should have such an FSR that there is no spectral overlap between their delay bands (here, we assume that all the delay elements have the same FSR), which also means the more channels (wavelengths) there are, the larger the FSR must be, and thus the smaller the size of the required ORRs. It will be further explained in Chapter 5 that in waveguide realization the minimum size of an ORR is limited by the length of applied tuning elements and the achievable minimum bend radius of the waveguide. Therefore, this OBFN scheme can only accommodate a limited number of inputs, which is a disadvantage for system scalability.

Compared to the single-wavelength OBFN shown in Figure 3.2, the above multiwavelength OBFN uses a common path for all DEs, but this does not change the delay requirements on each DE in the OBFN (i.e. maximum delay value and delay bandwidth) for the same antenna specifications. Thus, this OBFN scheme does not have advantages for system complexity. However, when an OBFN for a linear array antenna works with multiple wavelengths and its DEs have different FSRs, it is not necessary anymore to use as many DEs as in the previous OBFN scheme, or in other words, the number of the required DEs can be further reduced. To understand this idea, let us look at the example shown in Figure 3.11.

In this example, the OBFN is for a 4-element linear array antenna. The signal of AE 1 (on $\lambda_{1}$ ) is defined as the reference signal, thus the OBFN should provide different delays for the other $\mathrm{AE}$ signals (on $\lambda_{2}, \lambda_{3}$, and $\lambda_{4}$ ). In Figure 3.11 DE 1 
and DE 2 with different FSRs are the only two DEs used in the OBFN instead of three DEs with an equal FSR as shown in Figure 3.10. In this case, DE 1 and DE 2 have their individual delay bands at $\lambda_{2}$ (for AE 2) and $\lambda_{3}$ (for AE 3), respectively. In addition to that, both of them also repeat their delay bands at $\lambda_{4}$ where a delay level equal to the summation of the two individual delays of DE 1 and DE 2 is achieved without the requirement of a third DE. As explained in Section 3.2, for a linear array antenna the required delay level for an $\mathrm{AE}$ increases linearly with the distance between that $\mathrm{AE}$ and the reference $\mathrm{AE}$ (AE 1). Thus, when DE 1 and DE 2 are tuned individually to provide the required delays for $\mathrm{AE} 2$ and $\mathrm{AE} 3$, the required delay for $\mathrm{AE} 4$ is also achieved, since the required delay value for $\mathrm{AE} 4$ equals the summation of those for $\mathrm{AE} 2$ and $\mathrm{AE} 3$ according to the antenna geometry.
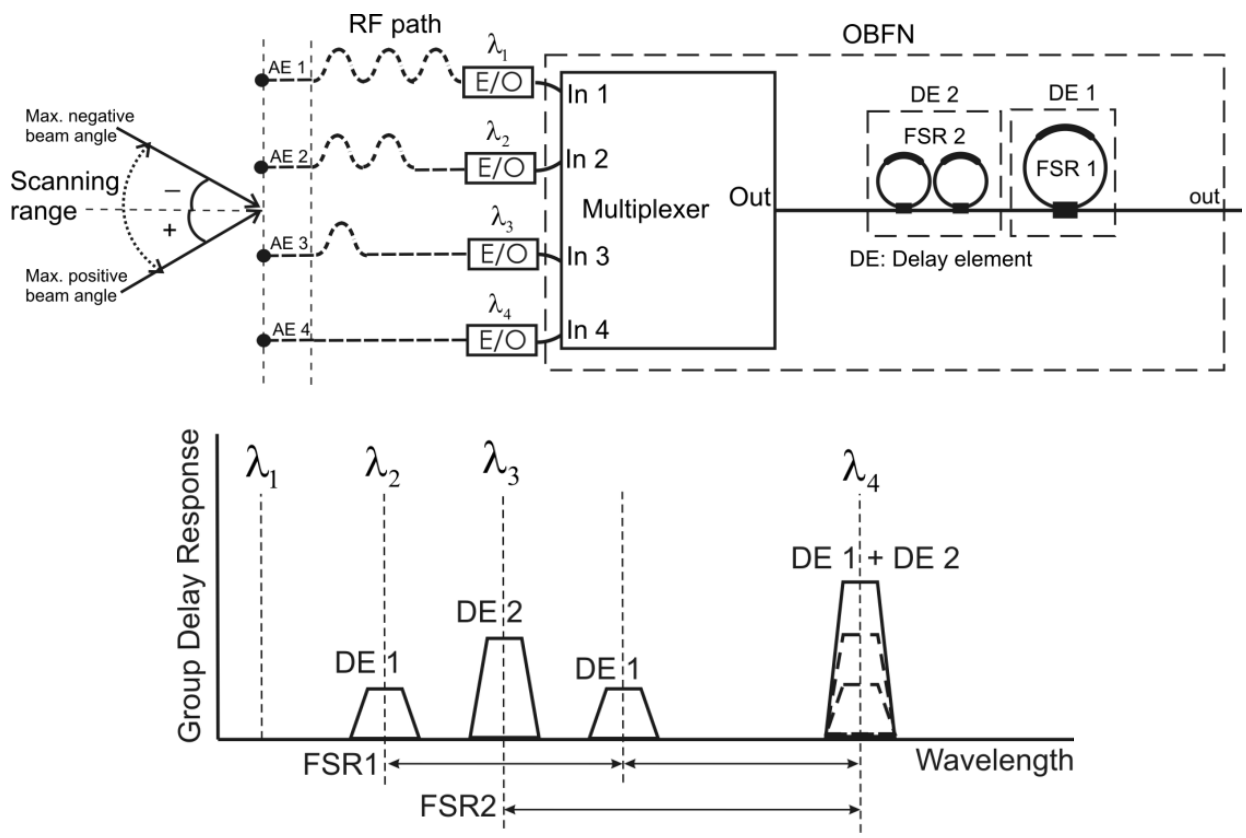

Figure 3.11: Drawings of 4-channel multi-wavelength OBFN with unequal-FSR delay elements (upper) and its group delay response (lower)

Compared to the previous example, this multi-wavelength OBFN does not need a dedicated DE for AE 4, and therefore the OBFN complexity is significantly reduced. Using this principle, a multi-wavelength OBFN with $N$ unequal-FSR DEs is able to provide a maximum of $2^{N}$ different delay values for the delay bands at different wavelengths. Those non-zero delay values at different wavelengths are generated either by the DEs individually or by means of the summation of the delay contributions of different DEs. Such an OBFN can be used to control a linear array antenna with up to $M=2^{N}$ AEs. 
The selection of the FSRs of the DEs and the optical carrier wavelengths can be done in various ways. However, to achieve this maximum of $2^{N}$ different delay values at different delay bands using $N$ DEs, there are two conditions which must be fulfilled when doing the selection. The first condition is that the FSR of a DE must not be an integer multiple of that of another DE; the second one is that the difference between the FSRs of two DEs must be larger than the bandwidth of the delay bands. This way, the DEs are able to generate their delay bands at different wavelength, and in the meantime there is no unwanted aliasing between those delay bands. Furthermore, the selected FSRs should be as small as possible to minimize the total optical bandwidth occupation of the system. For easy understanding, an example of the principle is given in Figure 3.12. In this example, the schematic of a cascade of three DEs with different FSRs and a possible arrangement of the delay bands and wavelengths of the optical carriers are illustrated. The FSRs of these three DEs are chosen to be prime-number multiples of a fixed bandwidth $\Delta f$, namely $\mathrm{FSR}_{1}=2 \Delta f$, $\mathrm{FSR}_{2}=3 \Delta f$, and $\mathrm{FSR}_{3}=5 \Delta f$, so that the first condition for FSR selection is fulfilled. Furthermore, $\Delta f$ is chosen to be larger than the bandwidth of the delay bands, so that the second condition is also fulfilled. In this case, eight different delay levels (including zero delay) are achieved at eight different wavelengths, and the total optical bandwidth occupation of the system is $15 \Delta f$.
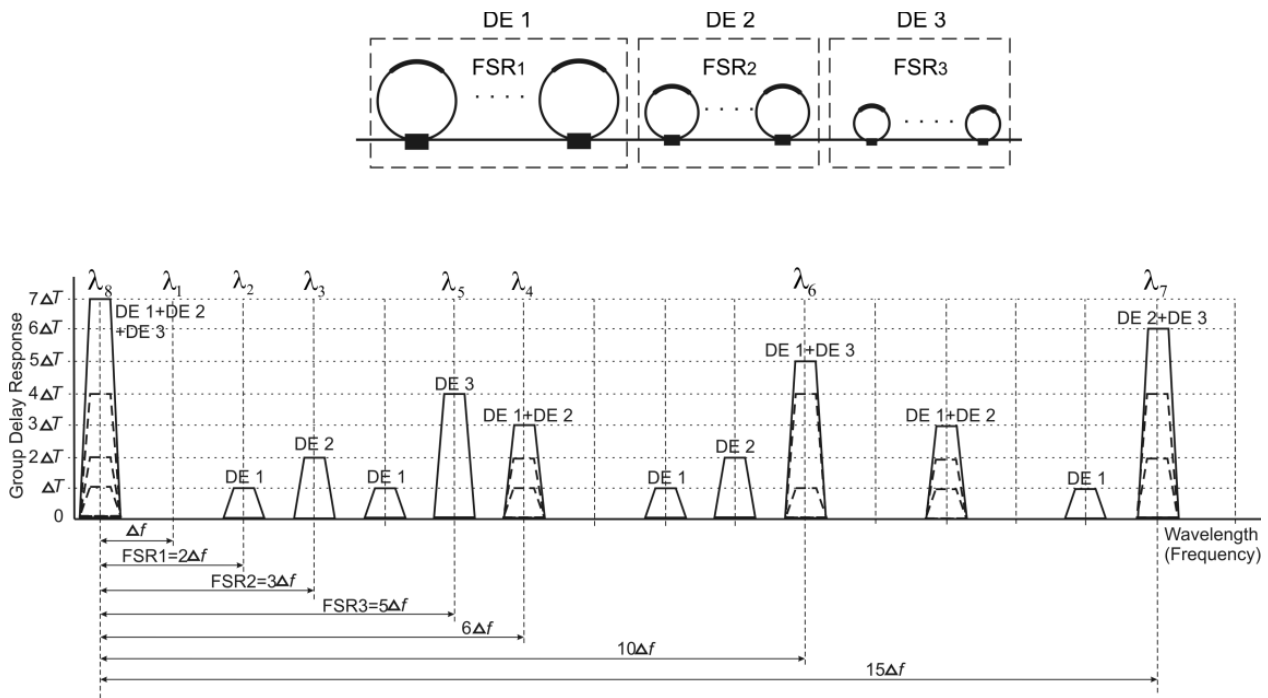

Figure 3.12: Schematic of a cascade of three DEs with different FSRs (upper); a possible arrangement of the delay bands and the wavelengths of the optical carriers (lower). 
Although the DEs in the multi-wavelength OBFN are tuned to have their delay bands at different wavelengths, the desired group delay response of a DE is still characterized by $\Delta f_{\mathrm{BW}}=\Delta f_{s}$ and $\bar{\tau}_{g}=-M_{-} T$, where $\Delta f_{s}$ is the signal bandwidth and $M_{-} T$ is the maximum time offset of the signal from the corresponding $\mathrm{AE}$ as in Eq. (3.5) - Eq. (3.7) for the asymmetrical parallel-structured OBFN. Based on the principle as shown in Figure 3.12, if $\mathrm{AE} 1$ of the linear array antenna is regarded as the reference $\mathrm{AE}$ for signal synchronization and the individual delay provided by $\mathrm{DE}$ $n$ is used for AE $2^{n-1}+1(n=1,2, \ldots, N)$, the number of necessary ORRs of DE $n$ and the total number of necessary ORRs of the $M$-channel $\left(M=2^{N}\right)$ multiwavelength OBFN can be calculated by

$$
\begin{gathered}
N_{n}=\left[\operatorname{Round}\left(\bar{\tau}_{g} \cdot \Delta f_{\mathrm{BW}}\right)+1\right]_{\mathrm{DE} n}=\left[\operatorname{Round}\left(-M_{-} T_{2^{n-1}} \cdot \Delta f_{s}\right)+1\right] \\
\begin{aligned}
N_{\mathrm{TT}}\left(N, d, \Delta \theta, \Delta f_{s}\right) & =\sum_{n=1}^{N} N_{n} \\
& =\sum_{n=1}^{N}\left[\operatorname{Round}\left(\frac{2 \sin (\Delta \theta / 2) \cdot n \cdot d}{c_{0}} \cdot \Delta f_{S}\right)+1\right]
\end{aligned}
\end{gathered}
$$

A numerical example corresponding to Eq. (3.21) is given in Example 3.7.

\section{Example 3.7}

Antenna specifications: $M=4 ; d=25 \mathrm{~cm} ; \Delta \theta=60^{\circ}$

Signal bandwidth: $\Delta f_{s}=1 \mathrm{GHz}$

Minimum number of delay elements: $N=2$

$N_{1}=\operatorname{Round}\left(\frac{2 \sin \left(30^{\circ}\right) \times 1 \times 25 \times 10^{-2}}{3 \times 10^{8}} \cdot 1 \times 10^{9}\right)+1=1+1=2$

$N_{2}=$ Round $\left(\frac{2 \sin \left(30^{\circ}\right) \times 2 \times 25 \times 10^{-2}}{3 \times 10^{8}} \cdot 1 \times 10^{9}\right)+1=2+1=3$

$N_{\mathrm{TT}}=\sum_{n=1}^{N} N_{n}=2+3=5$

Comparing the above example with the examples in Section 3.2, it is clear that for the same antenna specifications, the multi-wavelength OBFN requires less ORRs than the single-wavelength OBFN, resulting in lower OBFN complexity. However, the multi-wavelength scheme requires multiple laser sources and a multiplexer, which increases the system cost. Moreover, the arrangement of the FSRs of the DEs and the allocation of optical carrier wavelengths increases the difficulties in device realization and system implementation drastically as the number of OBFN inputs increases. Thus, the multi-wavelength OBFN can only allow a limited number of inputs, which is a disadvantage for system scalability. 


\section{Combined scheme}

Instead of using the schemes listed above individually, one can also combine two or more of them to construct an OBFN. The combined scheme could be more advantageous than the individual schemes, especially when the number of antenna elements is large. To give an example of this idea, let us consider an OBFN for a 16-element linear array antenna. One can use the multi-wavelength scheme to minimize the number of necessary ORRs in the OBFN. However, this scheme would require 16 lasers and 5 DEs with different FSRs, which means that a complicated wavelength and FSR arrangements must be performed to provide required delays for 16 spectrally separated signal channels. Moreover, the corresponding FSR requirements might demand very small ORRs, which bring challenges to the device realization technology. Thus, in this case using the multi-wavelength scheme alone would not be a practical choice.

Alternatively, one can use a combined scheme, for example to combine the binarytree scheme and multi-wavelength scheme. The antenna can be first divided into four smaller 4-element linear sub-arrays as shown in Figure 3.13.

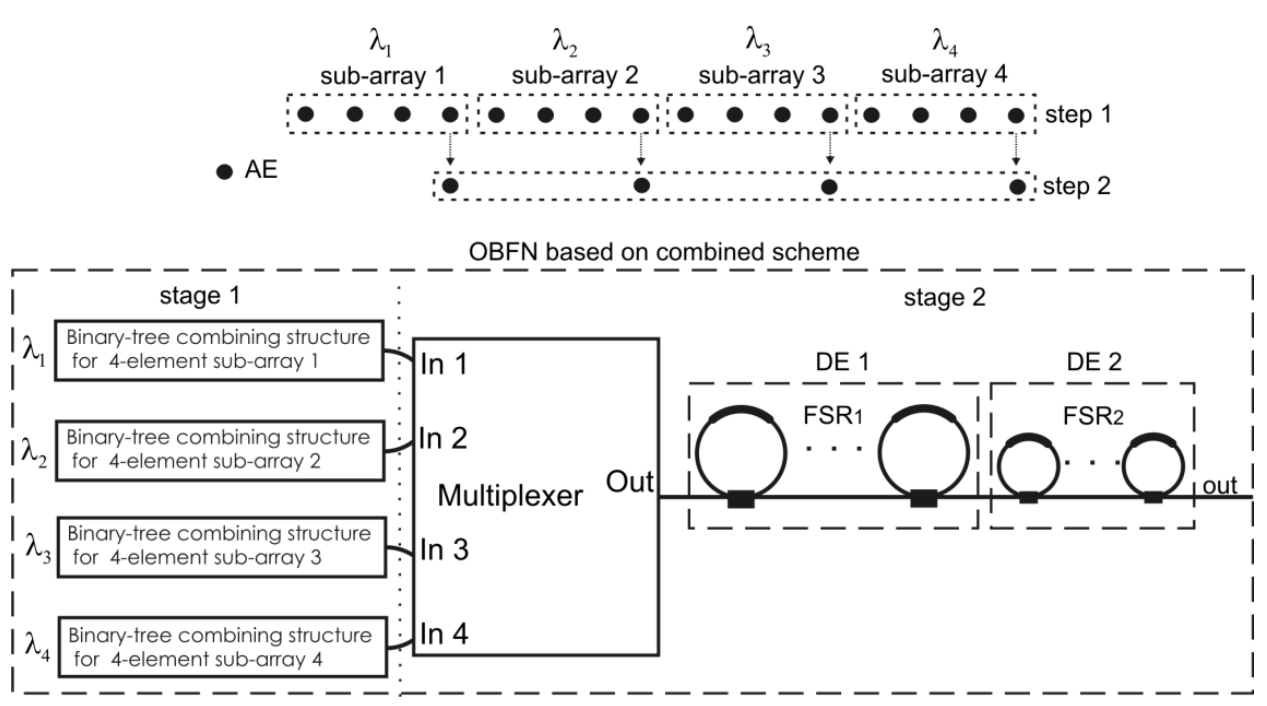

Figure 3.13: Drawing of the OBFN based on combined scheme

For each sub-array, the single-wavelength binary-tree structure scheme is used. The combination between the four sub-arrays is performed by the multi-wavelength scheme. As shown in Figure 3.13, the corresponding OBFN consists of two stages. The first stage has four 4-channel binary-tree combining structures, each of which works with a different wavelength. Then the second stage has four inputs on 
different wavelengths. Thus, only two unequal-FSR DEs are needed in the second stage as explained in the previous section. In this way, there will be a better balance between the number of ORRs, the number of laser sources, and the difficulties for device realization and system implementation. A numerical example showing the number of necessary ORRs of this combined scheme OBFN in comparison with that of a binary-tree structured OBFN is given in Example 3.8.

Furthermore, switches could also be added between the antenna and OBFN to further reduce the number of necessary ORRs by approximately a factor of two. However, this is only applicable if the eventual chip area and power consumption of the entire system (OBFN + switch matrix) is significantly lower.

\section{Example 3.8}

Antenna specifications: $M=2^{K}=16(K=4) ; d=25 \mathrm{~cm} ; \Delta \theta=60^{\circ}$

Signal bandwidth: $\Delta f_{s}=2 \mathrm{GHz}$;

Binary-tree structured OBFN:

$N_{\mathrm{TT}}=\sum_{k=1}^{K} \frac{M}{2^{k}} \cdot\left[\operatorname{Round}\left(\frac{2 \sin (\Delta \theta / 2) \cdot 2^{k-1} \cdot d}{c_{0}} \cdot \Delta f_{S}\right)+1\right]=70$

Combined scheme OBFN:

Sub-array dimensions: $M^{\prime}=2^{K^{\prime}}=4\left(K^{\prime}=2\right) ; d=25 \mathrm{~cm}$

Inter-sub-array distance: $d^{\prime}=4 d=100 \mathrm{~cm}$

No. of wavelengths: 4 (minimum No. of DEs for stage 2 of the OBFN: $N=2$ )

$N_{\text {TT }}=$

$4 \sum_{k=1}^{K^{\prime}} \frac{M^{\prime}}{2^{k}} \cdot\left[\operatorname{Round}\left(\frac{2 \sin (\Delta \theta / 2) \cdot 2^{k-1} \cdot d}{c_{0}} \cdot \Delta f_{S}\right)+1\right]+\sum_{n=1}^{N}\left[\operatorname{Round}\left(\frac{2 \sin (\Delta \theta / 2) \cdot n \cdot d^{\prime}}{c_{0}}\right.\right.$.

$\left.\left.\Delta f_{s}\right)+1\right]=62$

\subsection{Optical combiner and phase shifter}

\section{Optical combiner}

To achieve coherent optical combining, optical combiners are required in the OBFN. The simplest implementation of an optical combiner is a directional coupler (DC). However, it is difficult to realize a tunable DC with the used waveguide technology (the waveguide technology will be further explained in Chapter 5). An alternative solution for an optical combiner is to use a symmetrical MZI, which can couple the light from two input ports to a common output port with a certain coupling ratio determined by the phase difference between the two branches of the MZI. In practice a symmetrical MZI can easily be made tunable by adding an optical phase shifter in one of its arms. Moreover, it is tolerant to wavelength variations and 
fabrication errors [7], [8]. With tunable combiners one can remove the power differences between the OBFN channels so that the contribution of every antenna element to the output can be controlled. The tunable combiners can also be used to achieve amplitude tapering for beam forming purpose [9]-[11].

\section{Optical phase shifter}

In case a beam former system is based on the single-wavelength scheme where the optical carriers of all channels originate from a single laser, optical coherent combining has to be performed in the OBFN [1]. Consequently an optical phase synchronizer will be needed in one of the two input channels of each combiner in the OBFN to synchronize the phases of the two optical signals. Then, there are three kinds of tunable components in the OBFN, namely ORR-based delay elements, MZI-based optical combiners, and optical phase synchronizers. In practice all these three kinds of components achieve their tunability by means of using optical phase shifters (OPSs) which provides additional optical phase shifts in the optical paths. The full tuning range of the additional optical phase shift is $[0, \ldots, 2 \pi]$. The power coupling ratio of an MZI can be tuned by changing the phase difference between the two MZI arms as explained in Chapter 2. Actually OPSs are the only kind of tuning elements required for an ORR-based OBFN, which in fact simplifies the system design and device realization. An illustration of using OPSs for all tunable components of an OBFN is shown in Figure 3.14.

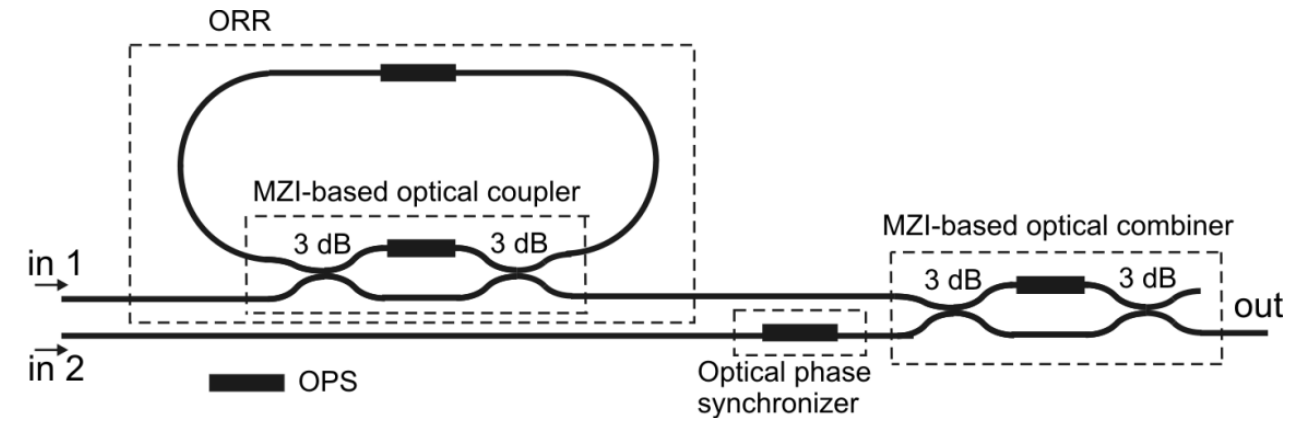

Figure 3.14: Schematic layout of a $2 \times 1$ OBFN using optical phase shifters for all tunable components

\subsection{Beam steering sensitivity}

Since all tuning elements of the OBFN are based on the OPSs, the beam angle of the beam former can be expressed in terms of the additional optical phase shifts of the OPSs. It will be further explained in Chapter 5 that the OPSs in the OBFN are realized by means of resistor-based heaters, and the phase shift of an OPS is 
controlled by the voltage supplied on the heater. Therefore, the beam angle of the beam former can also be expressed in terms of the voltages on the heaters. Knowing the relation between the beam angle and the voltages on the heaters, one can perform the beam steering by properly adjusting the voltages. The sensitivity of the beam steering to the heater voltage change determines the tuning precision of the beam angle, and is therefore an important property of the beam former system using the thermal tuning mechanism. To find out this beam steering sensitivity to the heater voltages, one first has to obtain the relation between the beam angle and the corresponding heater voltages according to the specifications of the antenna and the beam former system. To apprehend how this relation can be derived in an easy way, the simplified model of a $2 \times 1$ beam former system is used here as an example, which is shown in Figure 3.15.

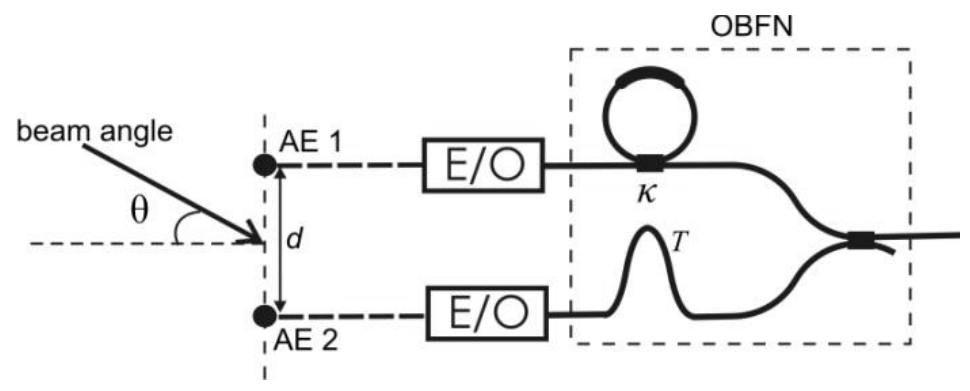

Figure 3.15: Schematic of a $2 \times 1$ beam former

In this simplified model, the OBFN has only one ORR in the upper channel as the delay element. The ORR has the roundtrip delay $T$ and provides a time delay $\tau_{g}$ for $\mathrm{AE} 1$ while $\mathrm{AE} 2$ is regarded as the reference AE. Then, based on Eq. (1.4) and Eq. (2.20), the beam angle $\theta$ can be given by

$$
\theta=\arcsin \left(\frac{\left(\tau_{\mathrm{g}}-T\right) \cdot \mathrm{c}_{0}}{d}\right)=\arcsin \left(\frac{(\tau-1) \cdot T \cdot \mathrm{c}_{0}}{d}\right)
$$

where $\tau=\tau_{g} / T$ represents the normalized group delay as explained in Chapter 2 . Further, we consider the ORR to have a low roundtrip loss and the effect of the loss on the delay can be neglected for simplicity. In this case the roundtrip transmission coefficient of the ORR equals $1(r=1)$, and $\tau_{p}$ can be given from Eq. (2.33) by

$$
\tau_{p}(\kappa)=\frac{1+\sqrt{1-\kappa}}{1-\sqrt{1-\kappa}}
$$

where $\kappa$ is the power coupling coefficient of the MZI-based coupler of the ORR. Now we combine Eq. (3.22) and Eq. (2.16) and assume for simplicity that the MZIbased coupler has perfect $3-\mathrm{dB}$ DCs $\left(\kappa_{\mathrm{DC}}=0.5\right)$, and there is no phase difference 
between the two arms of the MZI when the phase shift provided by the OPS is zero. Then the relation between $\tau$ and the additional optical phase shifts $\varphi$ of the OPS in the MZI can be given by

$$
\tau(\varphi)=\frac{1+\sqrt{1-\cos ^{2}(\varphi / 2)}}{1-\sqrt{1-\cos ^{2}(\varphi / 2)}}
$$

The plot of Eq.(3.23) is shown in Figure 3.16.

Since the phase shift $\varphi$ of the heater-based OPS has a linear relation with the dissipated power $P$ of the heater [6], the relation between $\varphi$ and the heater voltage $V$ can be given by

$$
\varphi=C \cdot P=C \cdot \frac{V^{2}}{R}
$$

where $R$ is the resistance of the heater and $C$ is the factor indicating the conversion efficiency from the dissipated power to the additional optical phase shift of the OPS.

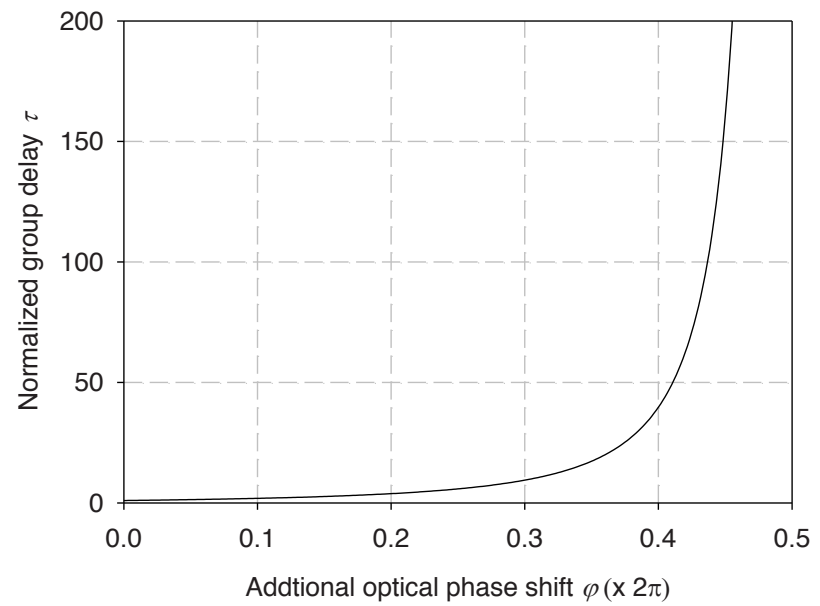

Figure 3.16: The relation between $\tau$ of the ORR and the additional optical phase shifts $\varphi$ of the OPS

Then by combining Eq. (3.23) and Eq. (3.24), the relation between the ORR delay and the voltage $V$ is obtained:

$$
\tau(V)=\frac{1+\sqrt{1-\cos ^{2}\left(C \cdot V^{2} / 2 R\right)}}{1-\sqrt{1-\cos ^{2}\left(C \cdot V^{2} / 2 R\right)}}
$$

Next, by combining Eq. (3.21) and Eq. (3.25), the relation between beam angle $\theta$ and the voltage $V$ is found: 


$$
\theta(V)=\sin ^{-1}\left(\left(\frac{1+\sqrt{1-\cos ^{2}\left(C \cdot V^{2} / 2 R\right)}}{1-\sqrt{1-\cos ^{2}\left(C \cdot V^{2} / 2 R\right)}}-1\right) \cdot T \cdot c_{0} / d\right)
$$

Now the beam steering sensitivity to the heater voltage can be obtained by taking the derivative of Eq. (3.26) with respect to $V$ :

$$
S(V)=\frac{\mathrm{d} \theta(V)}{\mathrm{d} V}
$$

In the above derivation process, several assumptions for the ideal situation are taken to simplify the mathematics, such as no loss, ideal DC, and temperature-independent resistance $R$. However, the principle of this simplified model is the same as that of the practical case. A numerical example of the estimation of beam steering sensitivity is given below.

\section{Example 3.9}

Antenna interelement distance: $d=1.18 \mathrm{~cm}$ (Ku-band antenna);

Beam steering range: $\left[0, \ldots, 60^{\circ}\right]$; Beam former architecture: Figure 3.15;

Roundtrip delay of the ORR: $T=0.07 \mathrm{~ns}$; Heater resistance: $R=800 \Omega$;

Heater conversion factor: $C=4 \pi$ (to give $2 \pi$ phase shift when $20 \mathrm{~V}$ is supplied on the heater); Speed of light in vacuum: $c_{0}=3 \times 10^{8} \mathrm{~m} / \mathrm{s}$.

Then using Eq. (3.26) and Eq. (3.27), $\theta(V)$ and $S(V)$ can be calculated, which are plotted in Figure 3.17 (a) and (b). It is shown that the beam steering sensitivity increases with the voltage supplied on the heater. When the beam angle is 60 degree (at $5 \mathrm{~V}$ ), the beam steering sensitivity is around 50 degree/ $\mathrm{V}$, or equivalently 0.05 degree/mV.

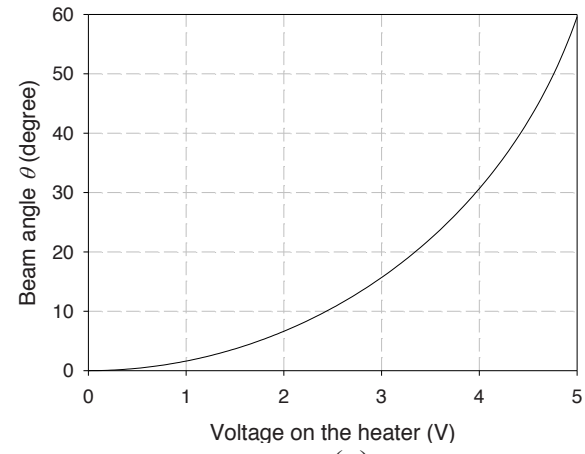

(a)

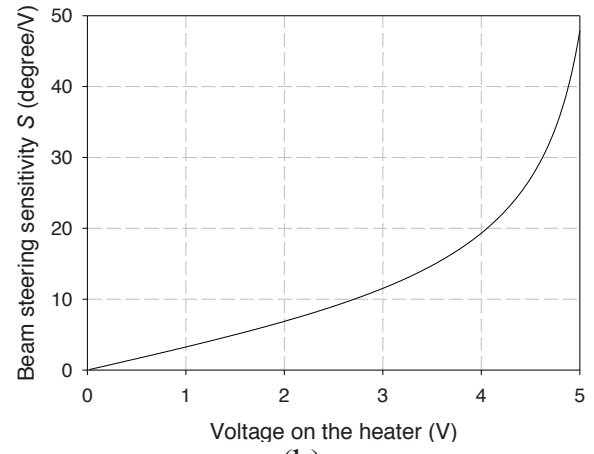

(b)

Figure 3.17: (a) calculated $\theta(V)$ and (b) calculated $S(V)$ of Example 3.9 


\subsection{Conclusions}

Basically an OBFN consists of DEs and combining circuitry. In the singlewavelength scheme, optical coherent combining will be performed in the OBFN, which also requires optical phase shifters for the synchronization of the optical phase between the OBFN channels. Based on the same antenna specifications, several OBFN structures have been compared and the asymmetrical binary-tree structured OBFN requires the least number of ORRs compared to its counterparts for the single-wavelength scheme, resulting in the lowest system complexity, and it can be used for both linear array (1-dimensional) and planar rectangular (2dimensional) PAAs. The switched scheme or multi-wavelength scheme may further reduce the required number of ORRs in the OBFN. But on the other hand they need additional signal processing circuitry or multiple expensive laser sources, and increase the difficulty of system implementation, especially when the number of OBFN inputs is large. Therefore, these two alternative schemes are only advisable to be used when they can significantly lower the eventual system complexity by reducing the number of ORRs significantly. Moreover, all the tuning elements in the OBFN can be realized by means of optical phase shifters. When the optical phase shifters are made by applying resistor-based heaters on the waveguide, the beam steering of the beam former is achieved by properly adjusting the supplied voltages on the heaters. A formula is derived to estimate the beam steering sensitivity to the voltage change. As a resume, a list of the structural features of different OBFN schemes for linear array antennas is given in Table 3.1.

\section{References}

[1] A. Meijerink, Coherence multiplexing for optical communication systems, $\mathrm{PhD}$ thesis, University of Twente, Enschede, 2005.

[2] Mohammad Ghavami, "Wideband Smart Antenna Theory Using Rectangular Array Structures," IEEE Trans. on signal processing, vol. 50, No. 9, pp. 21342151, Sep. 2002.

[3] Georgios I. Papadimitriou, Chrisoula Papazoglou, Andreas S. Pomportsis, "Optical Switching: Switch Fabrics, Techniques, and Architectures," $J$. Lightwave Technology, vol.21, No. 2, pp. 384-403, Feb. 2003.

[4] H. T. Mouftah, J. M. H. Elmirghani, Photonic Switching Technology, Wiley, Oct. 1998.

[5] S. V. Kartalopoulos, DWDM: Networks, Devices, and Technology, Wiley-IEEE Press, Oct, 2002.

[6] C. G. H. Roeloffzen, Passband flattened binary-tree structured add-drop multiplexers using SiON waveguide technology, $\mathrm{PhD}$ thesis, University of Twente, Enschede, 2002. 
[7] Okamoto, Katsunari, Fundamentals of Optical waveguides, 2ed Edition, Elsevier, 2006.

[8] Robert G. Hunsperger, Integrated optics: theory and technology, $5^{\text {th }}$ Edition, Springer, 2002.

[9] L. Josefsson, P. Persson, Conformal Array Antenna Theory and Design, WileyIEEE, Feb. 2006.

[10] R. Haupt, "Optimization of subarray amplitude tapers," IEEE Trans. on Antennas and Propagation, vol. 33, No. 8, pp. 846-850, Aug. 1985.

[11] Lu Yang, N. Ito, C. W. Domier, N. C. Luhmann, A. Mase, "18-40 GHz BeamShaping/Steering Phased Antenna Array System using Fermi Antenna," IEEE Trans. On Microwave Theory and Techniques, vol. 56, No. 4, pp.767-773, Apr. 2008.

Table 3.1: Structural features of different OBFN schemes for linear array antenna

\begin{tabular}{|c|c|c|c|}
\hline $\begin{array}{l}\text { OBFN scheme for } \\
M \text {-element linear array } \\
\text { antenna }\left(M=2^{K}\right) \\
\text { S.: Single-wavelength } \\
\text { M.: Multi-wavelength } \\
\text { s.: symmetrical } \\
\text { a.: asymmetrical }\end{array}$ & $\begin{array}{l}\text { No. } \\
\text { of } \\
\text { laser }\end{array}$ & $\begin{array}{l}\text { No. of necessary ORRs } \\
d \text { : antenna interelement distance } \\
\Delta \theta: \text { antenna beam scanning range } \\
k \text { : OBFN stage number } \\
N: \text { number of DEs } \\
\Delta f_{s}: \text { signal bandwidth } \\
c_{0}: \text { speed of light in vacuum }\end{array}$ & $\begin{array}{l}\text { Other signal } \\
\text { processing } \\
\text { modules }\end{array}$ \\
\hline S. s. parallel structure & 1 & $2 \sum_{m=1}^{M / 2}\left[\operatorname{Round}\left(\frac{\sin (\Delta \theta / 2) \cdot(M-m) d}{c_{0}} \cdot \Delta f_{s}\right)+1\right]$ & $\begin{array}{l}M-1 \\
\text { MZI-combiners; } \\
M-1 \text { OPSs. }\end{array}$ \\
\hline S. a. parallel structure & 1 & $\sum_{m=1}^{M}\left[\operatorname{Round}\left(\frac{2 \sin (\Delta \theta / 2) \cdot(m-1) d}{c_{0}} \cdot \Delta f_{s}\right)+1\right]$ & $\begin{array}{l}\text { Additional } \\
\text { channel } \\
\text { delay differences; } \\
M-1 \\
\text { MZI-combiners; } \\
M-1 \text { OPSs. }\end{array}$ \\
\hline $\begin{array}{l}\text { S. s. binary-tree } \\
\text { structure }\end{array}$ & 1 & $\sum_{k=1}^{K} \frac{M}{2^{k}} \cdot 2\left[\operatorname{Round}\left(\frac{\sin (\Delta \theta / 2) \cdot 2^{k-1} \cdot d}{c_{0}} \cdot \Delta f_{s}\right)+1\right]$ & $\begin{array}{l}M-1 \\
\text { MZI-couplers; } \\
M-1 \text { OPSs. }\end{array}$ \\
\hline $\begin{array}{l}\text { S. a. binary-tree } \\
\text { structure }\end{array}$ & 1 & $\sum_{k=1}^{K} \frac{M}{2^{k}} \cdot\left[\operatorname{Round}\left(\frac{2 \sin (\Delta \theta / 2) \cdot 2^{k-1} \cdot d}{c_{0}} \cdot \Delta f_{s}\right)+1\right]$ & $\begin{array}{l}\text { Additional } \\
\text { channel } \\
\text { delay differences; } \\
M-1 \\
\text { MZI-combiners; } \\
M-1 \text { OPSs. }\end{array}$ \\
\hline $\begin{array}{l}\text { S. a. binary-tree } \\
\text { structure + switch }\end{array}$ & 1 & $\sum_{k=1}^{K} \frac{M}{2^{k}} \cdot\left[\operatorname{Round}\left(\frac{\sin (\Delta \theta / 2) \cdot 2^{k-1} \cdot d}{c_{0}} \cdot \Delta f_{s}\right)+1\right]$ & $\begin{array}{l}M \times M \\
\text { switch matrix; } \\
M-1 \\
\text { MZI-combiners; } \\
M-1 \text { OPSs. } \\
\end{array}$ \\
\hline M. different FSRs & $M$ & $\sum_{n=1}^{N}\left[\operatorname{Round}\left(\frac{2 \sin (\Delta \theta / 2) \cdot n \cdot d}{c_{0}} \cdot \Delta f_{s}\right)+1\right]$ & $M \times 1$ multiplexer \\
\hline
\end{tabular}




\section{Chapter 4}

\section{Advanced signal processing techniques for optical ring resonator-based beam former systems}

\subsection{Introduction}

In the photonic beam former system Electrical/Optical (E/O) conversion and Optical/Electrical (O/E) conversion must be performed before and after the optical beam forming network (OBFN) respectively to convert the signals from antenna element (AE) between electrical and optical domain. These two conversions are implemented by means of optical modulation and detection, respectively. There are several well-known optical modulation and detection techniques available for these operations, such as optical double-sideband and single-sideband modulation; optical direct and balanced detection. In this chapter possible schemes for optical modulation and detection for the optical ring resonator-based (ORR-based) beam former system are described and compared with respect to system complexity and performance. After the comparison, a practical architecture for the beam former system is determined, which involves frequency down-conversion technique, filterbased optical single-sideband suppressed carrier (SSB-SC) modulation, and coherent optical balanced detection. The advantages of using these techniques are explained in the following sections. Then, some practical issues of the beam former system are discussed. At the end of this chapter, the functional design of the FLY and SKY beam former are described, which are both based on the practical architectures.

\subsection{Laser source}

The laser source is one of the essential components in the optical beam former system. It provides the optical carrier for the modulation of AE signals. For the ORR-based OBFN one important requirement for the laser source is that its spectral bandwidth should be much smaller than the bandwidth of the modulating signals. This requirement is meant to avoid unnecessary spectral broadening after the modulation process. As explained in Chapter 2, when the OBFN is based on ORRs, the OBFN complexity is directly related to the number of ORRs, and for the same delay value the necessary number of ORRs increases with the bandwidth 
requirement. Thus, larger bandwidth of the modulated optical signal will require more ORRs to achieve the same delay, and thus leads to higher OBFN complexity. For practical reasons the OBFN complexity should be kept as small as possible. Therefore, a narrowband laser source is required for the ORR-based beam former system.

For the beam former system using only one laser source, another important reason for using a narrowband laser source is related to the requirement of coherent optical combining which is mentioned in Chapter 3. For coherent optical combining, the laser source needs to generate an optical carrier with its coherence time much larger than the maximum delay difference between the OBFN channels, so that the optical carriers in all OBFN channels keep their phases correlated in the OBFN. The coherence time of the optical carrier is inversely-proportional to the linewidth of the laser source [1]. In this thesis the maximum delay difference under study is in the order of nanosecond, therefore a laser with the linewidth in the order of megahertz will be able to guarantee the sufficient coherence time of the optical carrier. Further explanation about this relation will be given in Section 7.2.1.

\subsection{Direct optical modulation}

The most straightforward way for $\mathrm{E} / \mathrm{O}$ conversion is to perform direct optical modulation of a semiconductor laser. However, direct modulation has two significant drawbacks. The first one is that modulating a laser source will cause variations not only in the amplitude but also in the frequency of the optical carrier, due to the frequency chirping effect [2]. Frequency chirping is undesired in the ORR-based beam former system since it also causes spectral broadening. The second drawback of direct modulation is that each $\mathrm{AE}$ requires a separate laser source. This would cause optical beat interference (OBI) in the system output due to the incoherent optical combining between the optical carriers provided by different laser sources [3], [4]. The OBI occupies the same frequency band as the signal. As a result, it would not be possible to filter out the OBI, and the output signal of the system would be corrupted. If each laser source works on a different wavelength and the wavelength intervals are much larger than the signal bandwidth, the OBI would not fall into the signal band, but as explained in Chapter 3, the multi-wavelength scheme is not only more costly but also difficult to implement for the PAA with many AEs. Therefore, it is practical to use only one laser source in the beam former system, if the corresponding complexity of the entire system is not a critical issue. As described in Chapter 3, in this case the light generated by the laser source should be split for all OBFN channels, and in each channel the E/O conversion can be achieved by means of external modulation. The external modulation techniques that 
could be used for the ORR-based beam former systems will be further explained in the following sections.

\subsection{Optical DSB modulation and direct optical detection}

As explained in the previous section, it is practical to apply external modulation for each beam former channel instead of direct modulation. In this case the most straightforward scheme for $\mathrm{E} / \mathrm{O}$ and $\mathrm{O} / \mathrm{E}$ conversion would be to use intensity modulation (IM) and direct detection (DD) [5]. IM can be performed by means of electro-absorption modulators (EAMs) [6] or Mach-Zehnder modulators (MZMs) [7] and DD by using a photodiode [8]. A sketch of this scheme is shown in Figure 4.1.

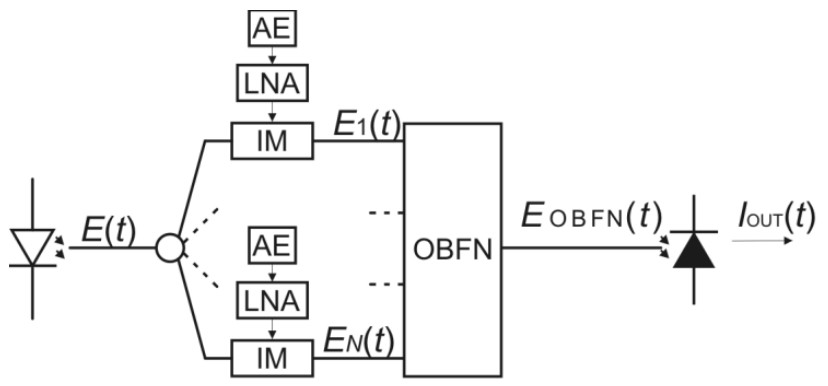

Figure 4.1: Photonic beam former system architecture with optical intensity modulation and direct optical detection

In each beam former channel, the intensity of the optical carrier is modulated by the signals from the corresponding AE. Thus, the square-law effect is involved in this process. The square-law effect means that even in case of perfectly linear IM, the optical spectrum of the output signal of the modulator would consist of the optical carrier line and an infinite number of sidebands. This is because when the intensity of a signal is varying at one frequency, the signal field would consist of a component of that frequency and an infinite number of harmonics [9]. For the beam former system MZMs are preferred to the EAMs since MZMs have better performance in linearity. Besides, MZMs are capable to provide chirp-free modulation [8]. The voltage applied to an MZM consists of a bias voltage and a modulating signal $s_{n}(t)$. The E/O conversion of an MZM is illustrated in Figure 4.2. In the MZM output the number of significant sidebands increases with increasing modulation depth. And increasing the number of significant sidebands means increasing the required OBFN bandwidth since the delay band should cover all significant sidebands to prevent serious linear distortion. 


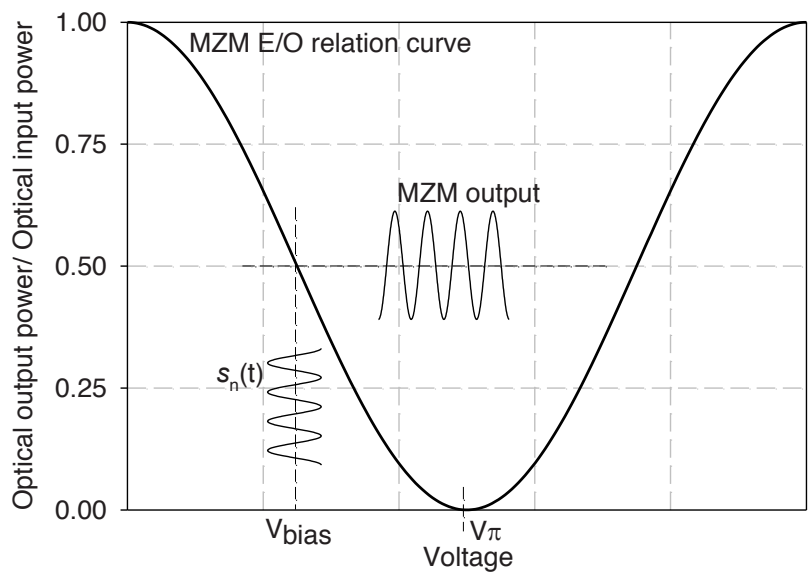

Figure 4.2: Illustration of $\mathrm{E} / \mathrm{O}$ relation of an MZM.

In order to keep the delay bandwidth requirement and the distortion in the OBFN as low as possible, the modulation depth should be such that only the first order sidebands are significant. Correspondingly, the ORR-based delay elements in the OBFN should provide a flat group delay response covering the optical carrier and the two sidebands. This is illustrated in Figure 4.3. The mathematical expression of this double-sideband (DSB) modulated optical signal and the corresponding system output are derived in the following part of this section. Since in this chapter we focus on the comparison of the beam former schemes in their signal spectrum characteristics instead of detailed system performance, some assumptions are used to simplify the derivations.

Imagine an $N$-element PAA receiver system. The received signal of each $\mathrm{AE}$ is first amplified by a low-noise amplifier (LNA), resulting in signals $s_{n}(t), n=1,2, \ldots, N$. When noise, interference, and channel distortion are ignored, these signals can be written as delayed versions of some desired signal $s(t)$, then $s_{n}(t)$ is given by

$$
s_{n}(t)=s\left(t-T_{n}\right)
$$

The delay values $T_{n}$ depend on the beam angle, geometry of the PAA, and possible differences in the lengths of the transmission lines. The desired signal $s(t)$ is written as a real bandpass signal [10]

$$
s(t)=a(t) \cos \left(2 \pi f_{\mathrm{RF}} t+\varphi(t)\right)
$$

Where $f_{\mathrm{RF}}$ is the carrier frequency of the RF signal, $a(t)$ and $\varphi(t)$ are the timedependent amplitude and phase, respectively. 


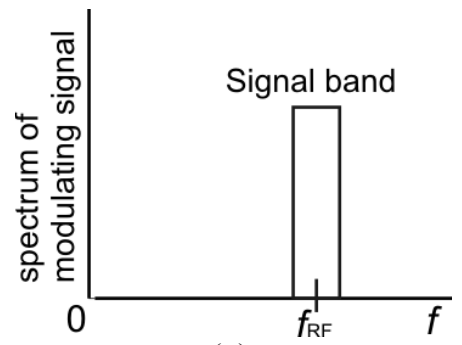

(a)

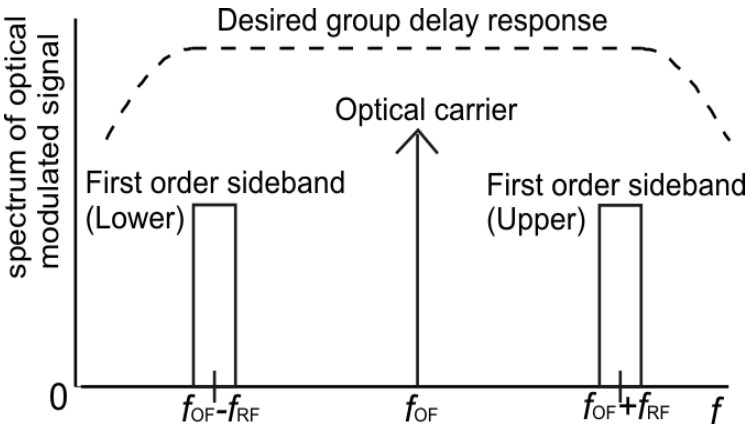

(b)

Figure 4.3: (a) drawing of the modulating signal spectrum; (b) the spectrum of modulated optical signal with desired group delay response.

To reveal the signal spectrum characteristics at different positions in the beam former system, a field description of the laser output is used here for the signal derivations instead of the intensity form. When the unmodulated laser signal has very narrow linewidth (approximated as monochromatic), it can be described by a complex bandpass signal $E_{L}$ with constant optical power $P=\left|E_{L}\right|^{2} / 2=\left(E_{L} \cdot E_{L}^{*}\right) / 2$ and optical frequency $f_{\mathrm{OF}}$, which is given below,

$$
E_{\mathrm{L}}=\sqrt{2 P} \exp \left(\mathrm{j} 2 \pi f_{\mathrm{OF}} t\right)
$$

Here, the laser intensity noise and phase noise are ignored. After the splitting of laser signal $E_{L}$, the portion for each channel enters the corresponding MZM for modulation. Since in this beam former scheme the desired RF signal is recovered from the OBFN output by means of DD whose operation requires the presence of the optical carrier in the modulated optical signal [5], the MZM should keep a significant optical carrier in its output, or in other words, the MZM bias points that results in strong optical carrier suppression (around $V_{\pi}$ in Figure 4.2) should be avoided. Assume that the bias point and the modulation depth of the MZM are such that second and higher order sidebands are much lower compared to the two first 
order sidebands, then the MZM can be modeled as an ideal amplitude modulator [11], which relates its optical output $E_{\text {out }}(t)$ to its optical input $E_{\text {in }}(t)$ by

$$
E_{\text {out }}(t)=q_{n}\left[1+\gamma_{n} \frac{s_{n}(t)}{\left|s_{n}(t)\right|_{\text {max }}}\right] E_{\text {in }}(t)
$$

where $q_{n}$ is an amplitude coefficient determined by the bias point and insertion loss of the MZM, and $\gamma_{n}$ is the modulation index $\left(\gamma_{n} \ll 1\right)$.

Assume a laser power splitting loss $L_{\mathrm{SP}}$, then the modulated optical signals $E_{n}(t)$ can be given by

$$
\begin{aligned}
E_{n}(t)= & \frac{q_{n}}{\sqrt{L_{\mathrm{SP}}}}\left[1+\gamma_{n} \frac{s_{n}(t)}{\left|s_{n}(t)\right|_{\mathrm{max}}}\right] E_{\mathrm{L}}(t) \\
= & \sqrt{\frac{2 P}{L_{\mathrm{SP}}}}\left[q_{\mathrm{n}} \exp \left(\mathrm{j} 2 \pi f_{\mathrm{OF}} t\right)\right. \\
& +\frac{1}{2} \rho_{n} a\left(t-T_{n}\right) \cdot\left\{\exp \left(\mathrm{j} 2 \pi\left[\left(f_{\mathrm{OF}}+f_{\mathrm{RF}}\right) t-f_{\mathrm{RF}} T_{n}\right]+\mathrm{j} \varphi\left(t-T_{n}\right)\right)\right. \\
& \left.\left.+\exp \left(\mathrm{j} 2 \pi\left[\left(f_{\mathrm{OF}}-f_{\mathrm{RF}}\right) t+f_{\mathrm{RF}} T_{n}\right]-\mathrm{j} \varphi\left(t-T_{n}\right)\right)\right\}\right]
\end{aligned}
$$

where $\rho_{n} \triangleq q_{n} \gamma_{n} /|a(t)|_{\max }$.

To know if the desired RF signal can be obtained after DD, the output of the OBFN should first be derived. When optical losses and the ripples of the delay elements are neglected, and coherent combining is performed which gives no combining loss, the transfer function from input $n$ to the output of the OBFN can be written as

$$
H_{n}(f)=\exp \left\{\mathrm{j} \Phi_{\mathrm{n}}(f)\right\}
$$

where the desired phase response in the frequency range of interest is given by

$$
\Phi_{\mathrm{n}}(f)=\Phi_{\mathrm{n}}\left(f_{\mathrm{OF}}\right)-2 \pi \int_{f_{\mathrm{OF}}}^{f} \tau_{\mathrm{n}}(v) \mathrm{d} v=\Phi_{\mathrm{n}}\left(f_{\mathrm{OF}}\right)-2 \pi\left(f-f_{\mathrm{OF}}\right) \tau_{n}
$$

where $\tau_{n}$ is the generated delay of OBFN channel $n$, which follows $\tau_{n}>0$, $\tau_{n}+T_{n}=T_{\mathrm{CO}}$, where $T_{\mathrm{CO}}$ is considered to be the common total delay for all beam former channels.

Based on Eq. (4.5) - Eq. (4.7), the optical signal at the output of the OBFN can be written as 


$$
\begin{aligned}
E_{\mathrm{OBFN}}(t)= & \sqrt{\frac{2 P}{L_{\mathrm{SP}}}} \exp \left(\mathrm{j} 2 \pi f_{\mathrm{OF}} t\right) \cdot \\
& \sum_{n=1}^{N} \exp \left(\mathrm{j} \Phi_{\mathrm{n}}\left(f_{\mathrm{OF}}\right)\right) \cdot\left[q_{n}+\rho_{n} a(t) \cos \left(2 \pi f_{\mathrm{RF}} t+\varphi(t)\right)\right]
\end{aligned}
$$

where the effect of $T_{\mathrm{CO}}$ on the RF phase is not taken into account since it is the same for all the channels. The corresponding output power can be calculated by

$$
P_{\mathrm{OBFN}}=\frac{1}{2}\left|E_{\mathrm{OBFN}}(t)\right|^{2}=\frac{1}{2} E_{\mathrm{OBFN}} E_{\mathrm{OBFN}}^{*}
$$

Obviously, the maximum signal amplitude (power) is achieved when the optical signals are combined in phase, which means besides the delay synchronization of the AE signals, the optical carrier phases $\Phi_{\mathrm{n}}\left(f_{\mathrm{OF}}\right)$ should all have the same value. After the coherent combining, the $\mathrm{O} / \mathrm{E}$ conversion is performed by detecting the output power of the OBFN with a single-end photodiode. If the photodiode has a responsively $R_{\mathrm{PD}}$, the output current of the beam former system is given by

$$
\begin{aligned}
I_{\mathrm{OUT}}(t)= & R_{\mathrm{PD}} P_{\mathrm{OBFN}}=\frac{1}{2} R_{\mathrm{PD}}\left|E_{\mathrm{OBFN}}(t)\right|^{2}=\frac{R_{\mathrm{PD}} P}{L_{\mathrm{SP}}}\left[\mathrm{Q}_{N}^{2}+\frac{1}{2} \mathrm{P}_{N}^{2} a^{2}(t)+\right. \\
& \left.2 \mathrm{Q}_{N} \mathrm{P}_{N} a(t) \cos \left(2 \pi f_{\mathrm{RF}} t+\varphi(t)\right)+\frac{1}{2} \mathrm{P}_{N}^{2} a^{2}(t) \cos \left(4 \pi f_{\mathrm{RF}} t+2 \varphi(t)\right)\right]
\end{aligned}
$$

where $Q_{N} \triangleq \sum_{n=1}^{N} q_{n}$ and $P_{N} \triangleq \sum_{n=1}^{N} \rho_{n}$.

Looking in the bracket of Eq. (4.10), it is clear that the output current consists of a DC term (first term), a baseband term (second term), a desired RF term (third term), and a double-frequency term (last term), as illustrated in Figure 4.4.

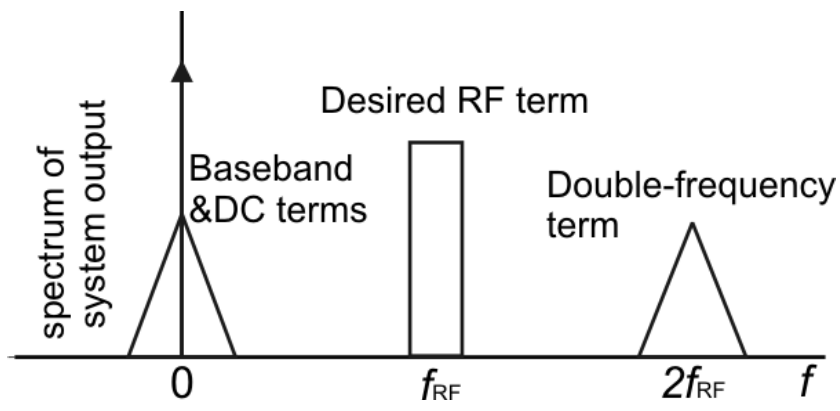

Figure 4.4: Drawing of the spectrum of system output current $I_{\text {out }}(t)$, in case of DSB optical modulation and single-end direct optical detection. 
The DC term corresponds to the power of the optical carrier, whereas the desired RF term results from beating between the carrier and the sidebands. The baseband term and double-frequency term result from self-beating and mutual beating of the sidebands, respectively, and therefore have a spectral width which is twice the RF bandwidth. It can be verified that the relative bandwidth of the RF signal must be less than one octave, so that the terms will not spectrally overlap, and the desired term can be selected by means of bandpass filtering.

In this scheme the minimum optical bandwidth required in the OBFN is equal to twice the maximum frequency of the modulating RF signal as shown in Figure 4.3. In case of high-frequency phased array antennas (PAAs), for instance a linear array antenna for $30 \mathrm{GHz} \mathrm{K}$-band satellite communication, several disadvantages exist in the corresponding beam former system. First of all, fast modulators and detectors (whose operation bandwidth must be larger than $30 \mathrm{GHz}$ ) must be used for the $\mathrm{E} / \mathrm{O}$ and $\mathrm{O} / \mathrm{E}$ conversion, which will heavily increase the system cost. Secondly, a very large number of ORRs is needed to meet the bandwidth requirement ( $>60 \mathrm{GHz}$ ), which will result in an excessively complex OBFN and also immensely complex tuning electronics because of the excessive wiring to all the tuning elements. A numerical calculation showing this disadvantage is given in Example 4.1. Moreover, when the number of ORRs is large, it will be difficult to fit them in a chip with limited area (more details about device realization will be given in Chapter 5).

Example 4.1

Antenna type: linear array

Antenna diameter: $d=1 \mathrm{~m}$

Beam scanning range: $\Delta \theta=60^{\circ}$

Bandwidth of modulated optical signal: $\Delta f_{s}=60 \mathrm{GHz}$

Maximum time difference between two most outer AEs:

$T_{\text {max }}=\frac{d \cdot \sin (\Delta \theta / 2)}{c_{0}}$

Based on the Eq. (2.44), the required number of ORR for compensating $T_{\max }$ over $\Delta f_{S}$ can be estimated by

$N=\operatorname{Round}\left(T_{\max } \cdot \Delta f_{S}\right)+1=\operatorname{Round}\left(\frac{1 \cdot \sin \left(30^{\circ}\right)}{3 \times 10^{8}} \times 60 \times 10^{9}\right)+1=101$

\subsection{RF frequency down conversion}

As explained in Chapter 2 and 3, the system complexity is related to the bandwidth requirement for the OBFN. To lower this bandwidth requirement means to reduce the bandwidth of the modulated optical signal. One way to achieve this is to apply frequency down conversion (FDC) to an intermediate frequency (IF) range prior to 
E/O conversion [12]. This can be performed by means of mixing the AE signals $s_{n}(t)$ with a common local oscillator (LO) signal $\cos \left(2 \pi f_{\mathrm{LO}} t\right)$, and low-passfiltering. Then the resulting signal for every $\mathrm{AE}$ is given by

$$
s_{n}^{\prime}(t)=a\left(t-T_{n}\right) \cos \left(2 \pi f_{\mathrm{IF}}\left(t-T_{n}\right)-2 \pi f_{\mathrm{LO}} T_{n}+\varphi\left(t-T_{n}\right)\right)
$$

where $f_{\mathrm{IF}}=f_{\mathrm{RF}}-f_{\mathrm{LO}}$ is the new center frequency. Consequently, the sidebands in Figure 4.3 will now be closer to each other. When $f_{\mathrm{IF}}$ is made much lower than $f_{\mathrm{RF}}$, the total optical bandwidth of the modulated optical signal will be considerably reduced. An additional advantage obtained from FDC is that lower-speed optical modulators and detectors can be used. However, two disadvantages exist in this approach. For one thing, mixing mutually delayed AE signals with a non-delayed oscillator signal results in a phase offset $\left(2 \pi f_{\mathrm{LO}} T_{n}\right)$ that no longer corresponds to the delay offset. In other words, the down-converted signals are no longer delayed versions of one another $\left(s_{n}^{\prime}(t) \neq s^{\prime}\left(t-T_{n}\right)\right)$. The OBFN output becomes

$$
\begin{aligned}
E_{\mathrm{OBFN}}(t)= & \sqrt{\frac{P}{2 L_{\mathrm{SP}} L_{\mathrm{CO}}}} \exp \left(j 2 \pi f_{\mathrm{OF}} t\right) \cdot \sum_{n=1}^{N} \exp \left(j \Phi_{n}\left(f_{\mathrm{OF}}\right)\right) . \\
& {\left[1+\rho_{n} a(t) \cos \left(2 \pi f_{\mathrm{IF}} t-2 \pi f_{\mathrm{LO}} T_{n}+\varphi(t)\right)\right] }
\end{aligned}
$$

As a result, FDC has resulted in IF phase offsets $\left(2 \pi f_{\mathrm{LO}} T_{n}\right)$ after delay equalization, which may result in destructive interference, and hence ruin the correct operation of the PAA. This could be avoided by phase-shifting the individual LO signals by values $2 \pi f_{L O} T_{n}$, but this would complicate the beam forming circuit, as $T_{n}$ depends on the direction of the incoming beam. A second problem arises when the AE signals are down-converted to an IF range with a relative bandwidth of more than one octave. Direct detection will then make the IF beating term spectrally overlap with the baseband and double-frequency terms, resulting in intermodulation distortion (IMD) as shown in Figure 4.5. One way to cope with this is to keep the modulation index small, so that the baseband and double-frequency terms are small compared to the desired term. However, this lowers the dynamic range of the system.

\subsection{Optical SSB-SC modulation}

A more effective way to reduce the bandwidth of the modulated optical signal is to remove one of the sidebands and the optical carrier by means of optical singlesideband suppressed-carrier (SSB-SC) modulation, so that only one sideband remains in the optical spectrum [13]. In this case the optical bandwidth equals the 


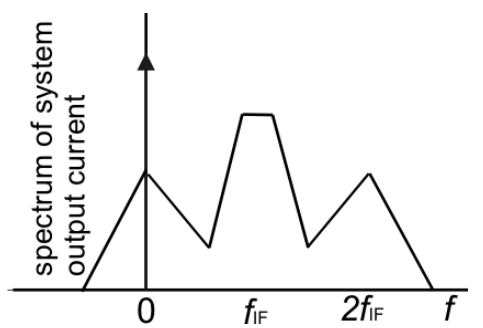

Figure 4.5: The spectrum of the beam former system output in case of frequency down conversion prior to optical DSB modulation, with a relative IF bandwidth larger than one octave.

RF bandwidth, which is the smallest that can be achieved in the sub-bands prior to $\mathrm{E} / \mathrm{O}$ conversion. Assume that the lower sideband and optical carrier are removed, then based on Eq. (4.5) the mathematical expression of the SSB-SC signal can be written as

$$
\begin{aligned}
E_{n}(t)= & \frac{1}{2} \sqrt{\frac{2 P}{L_{\mathrm{SP}}}} \rho_{n} a\left(t-T_{n}\right) \cdot \\
& \exp \left(j 2 \pi\left[\left(f_{\mathrm{OF}}+f_{\mathrm{RF}}\right) t-f_{\mathrm{RF}} T_{n}\right]+j \varphi\left(t-T_{n}\right)\right)
\end{aligned}
$$

Its spectrum and the corresponding desired group delay response are illustrated in Figure 4.6.

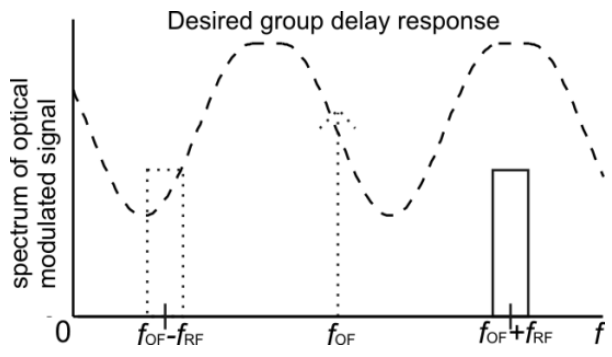

Figure 4.6: Spectrum of optical SSB-SC modulation

With this approach the bandwidth requirement for OBFN is considerably reduced compared to Figure 4.2. Suppose the RF bandwidth is, for example, one tenth the RF carrier frequency, then the resulting optical bandwidth after removing one sideband and optical carrier will be even less than 5 percent of the DSB bandwidth. Obviously, applying SSB-SC modulation can significantly reduce the required number of ORRs for the OBFN and hence reduce the complexity of the beam former 
system. In case of optical SSB-SC modulation the output of the OBFN can be written as

$$
\begin{aligned}
E_{\mathrm{OBFN}}(t)= & \frac{1}{2} \sqrt{\frac{2 P}{L_{\mathrm{SP}}}} a(t) \cdot \\
& \sum_{n=1}^{N} \rho_{n} \exp \left(j 2 \pi\left(f_{\mathrm{OF}}+f_{\mathrm{RF}}\right) t+j \varphi(t)+j \Phi_{\mathrm{n}}\left(f_{\mathrm{OF}}\right)\right)
\end{aligned}
$$

where the common delay $T_{\mathrm{CO}}$ has once again been omitted.

Optical SSB-SC modulation requires coherent detection, which implies that the unmodulated optical carrier has to be re-inserted prior to optical detection. This can be done by routing one output signal of the splitter after the laser around the OBFN, and then combining it with the output signal of the OBFN $E_{\mathrm{OBFN}}$ using a coupler. If the same single-ended detection is performed as in the DSB modulation scheme, in the output there will be no double frequency term that comes from the beating between two sidebands, however, there will be again DC and a baseband term along with the desired RF signal due to the re-inserted optical carrier and self-beating of the sideband, and therefore bandpass filtering must be performed to remove them. The output spectrum is shown in Figure 4.7. The advantage of this output signal compared to that from the DSB modulation scheme is that only one beating product at the desired RF frequency results, whereas DSB-modulated signals give two beating products at the desired RF frequency, which are generally not in phase in case of chromatic dispersion, resulting in RF power fading. For this reason, optical SSB modulation has been previously proposed as a means to overcome the bandwidth-limiting effect of chromatic dispersion in single-mode fiber-based Radioover-fiber (RoF) transmission systems [14] and chirped fiber grating-based optical beam forming system [15]. However, for the beam former system the major advantage of SSB-SC modulation is its bandwidth efficiency, which results in lower requirement on the number of ORRs. The implementation of optical SSB-SC modulation will be further discussed in Section 4.9.

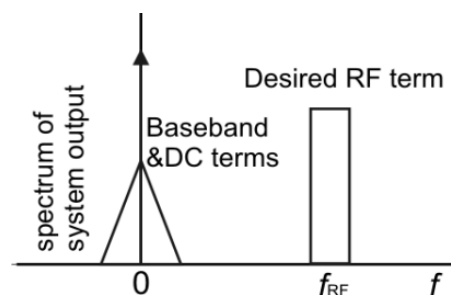

Figure 4.7: Drawing of the spectrum of system output current $I_{\text {out }}(t)$, in case of SSB optical modulation and single-end coherent optical detection. 


\subsection{Balanced coherent optical detection}

To further improve the system, balanced coherent optical detection can be applied to replace the single-ended detection. The corresponding beam former system scheme is shown in Figure 4.8. In this scheme, a balanced detector is placed after the $2 \times 2$ coupler which is used to combine the unmodulated optical carrier and OBFN output, and the two photodiodes detect the optical powers from the two coupler output ports separately $\left(P_{1}, P_{2}\right)$ and then subtract their output currents from each other. When the coupler is assumed lossless and has uniform splitting, and the photodiodes are assumed to have identical responsivities $R_{\mathrm{PD}}$, then the output current $I_{\mathrm{OUT}}$ of the balanced detector can be given by

$$
\begin{aligned}
I_{\mathrm{OUT}}(t) & =\frac{1}{2} R_{\mathrm{PD}}\left[P_{1}-P_{2}\right] \\
& =\frac{1}{2} R_{\mathrm{PD}}\left[\left|\frac{E_{\mathrm{OBFN}}(t)}{\sqrt{2}}+\frac{\mathrm{j} E_{\mathrm{L}}(t)}{\sqrt{2 L_{\mathrm{SP}}}}\right|^{2}-\left|\frac{\mathrm{j} E_{\mathrm{OBFN}}(t)}{\sqrt{2}}+\frac{E_{\mathrm{L}}(t)}{\sqrt{2 L_{\mathrm{SP}}}}\right|^{2}\right] \\
& =\frac{\mathrm{j} R_{\mathrm{PD}}}{2 \sqrt{L_{\mathrm{SP}}}}\left[E_{\mathrm{OBFN}}^{*}(t) E_{\mathrm{L}}(t)-E_{\mathrm{OBFN}}(t) E_{\mathrm{L}}^{*}(t)\right]
\end{aligned}
$$

Insert Eq. (4.14) into Eq. (4.15), and assume that the optical phases $\Phi_{\mathrm{n}}\left(f_{\mathrm{OF}}\right)$ are synchronized to a common value $\Phi$, then the Eq. (4.15) reduces to

$$
I_{\mathrm{OUT}}(t)=\frac{2 R_{\mathrm{PD}} P P_{\mathrm{N}}}{L_{\mathrm{SP}}} a(t) \sin \left(2 \pi f_{\mathrm{RF}} t+\varphi(t)+\Phi\right)
$$

Note that the desired RF terms in the two arms of the balanced detector are antiphase, so that the effect of the subtraction behavior of the balanced detector (see Eq. (4.15)) is that these desired RF terms actually are added up instead of being subtracted from each other, unlike the undesired DC and the double-frequency terms. The spectrum of this $I_{\text {OUT }}(t)$ is illustrated in Figure 4.9.

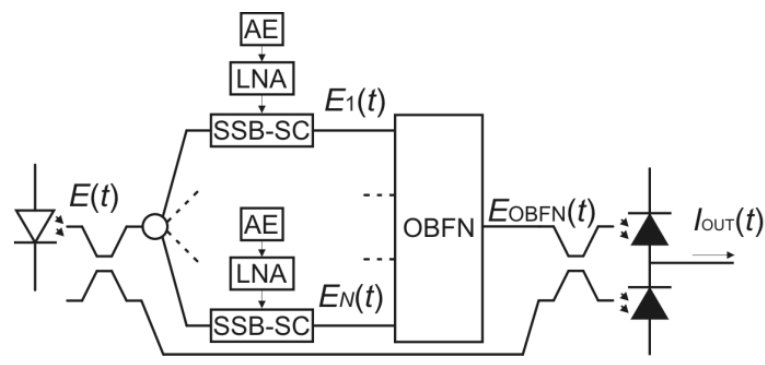

Figure 4.8: Photonic beam former system architecture with optical SSB-SC modulation and balanced coherent optical detection 


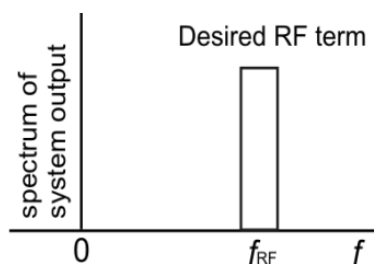

Figure 4.9: Drawing of the spectrum of system output current $I_{\text {out }}(t)$, in case of SSB optical modulation and balanced coherent optical detection.

Obviously, balanced detection cancels the DC and baseband terms in Eq. (4.10), which correspond to the left part of the spectrum in Figure. 4.4. The doublefrequency term is not there because the optical signal has only one sideband. Therefore only the desired RF term remains. Owing to this absence of baseband and double frequency terms in the output, the beam former system can deal with a relative RF bandwidth of more than one octave, without having the IMD problem as shown in Figure 4.5. An additional advantage of balanced detection is that the effect of optical relative intensity noise (RIN) is significantly reduced owing to the cancelation of DC and baseband terms. Generally, RIN and shot noise [16] are the two kinds of dominant noises in an analog-optical link [17], thus reducing the effect of RIN will surely benefit the signal dynamic range of the system [18]. Therefore, balanced detection is strongly preferred over single-ended detection.

\subsection{Combination of FDC and optical SSB-SC modulation}

An even more advantageous scheme for the $\mathrm{E} / \mathrm{O}$ and $\mathrm{O} / \mathrm{E}$ conversion is to combine FDC, SSB-SC modulation, and balanced coherent optical detection. As explained in the previous sections, FDC before optical modulation lowers the bandwidth requirement on the optical modulators and detector, optical SSB-SC modulation minimizes the OBFN bandwidth requirement, and balanced coherent optical detection avoids IMD and enhances signal dynamic range of the system. Additionally, in this scheme the drawback of the previous FDC scheme is circumvented: the IF phase offsets due to FDC prior to optical modulation can be removed. Since the output signal after the balanced detection contains only one beating product at the desired frequency, its phase is simply determined by the optical phase difference between the modulated optical signals and the re-inserted optical carrier signal. Therefore, IF phase offsets can be canceled by means of simple optical phase shifters in the OBFN channels. Moreover, since in this scheme a relative IF bandwidth of more than one octave will no longer result in the IMD problem as shown in Figure 4.5, the IF range can be further shifted towards the baseband, which even further relaxes the bandwidth requirements on the optical modulators and detector. 


\subsection{Implementation of optical SSB-SC modulation}

To implement optical SSB-SC modulation in the beam former system, two possible approaches are described below:

1) Filter-based approach: this is the most straightforward way to achieve optical SSB-SC modulation, which consists of two steps. In the first step, optical DSB modulation is performed by means of an MZM as described in Section 4.4. Then in the second step, a dedicated optical sideband filter (OSBF) is used to filter out the carrier and one of the sidebands. In this approach the optical carrier can also be suppressed inherently by MZM when the bias close to $V_{\pi}$ is used (see Figure 4.2), then the OSBF needs only to filter out one of the sidebands, which will relax the slope requirements on the OSBF characteristic.

2) Approach based on the phase-shift method: this approach is based on the classical SSB generation technique, where two quadrature carriers are modulated by a modulating signal and its Hilbert transform, respectively. Several optical implementations are known, such as using a dual-electrode MZM [19], hybrid amplitude and phase modulation [20], two parallel MZMs [21], and a Sagnac loop with a unidirectional [22] or bidirectional [23] travelling-wave MZM. The double MZM seems to be the only scheme to offer distortion-free SSB-SC modulation, but on the other hand it requires two MZMs per AE.

For our beam former system, the filter-based approach appears to be a practical choice. The OSBFs can be placed directly after the MZMs, but then every AE would require its own OSBF. However, since the OSBF and OBFN are both linear devices, their order can be reversed, so that only one common OSBF is required. A practical OSBF implementation is an MZI with an ORR in one of its arms [24]. This filter architecture provides flattened passbands and broad stopbands, which matches this application. Besides, this filter consists of the same kinds of building blocks as the OBFN (ORRs and MZIs), thus in practice both OBFN and the OSBF can be realized in one chip, which enables the system integration. The system architecture in case of filter-based SSB-SC modulation and balanced coherent detection is shown in Figure 4.10.

\subsection{Practical issues}

After the discussion in the preceding sections, an advantageous scheme for the $\mathrm{E} / \mathrm{O}$ and $\mathrm{O} / \mathrm{E}$ conversion in the photonic beam former system has been achieved. However, there are still some practical issues that have influence on the system 


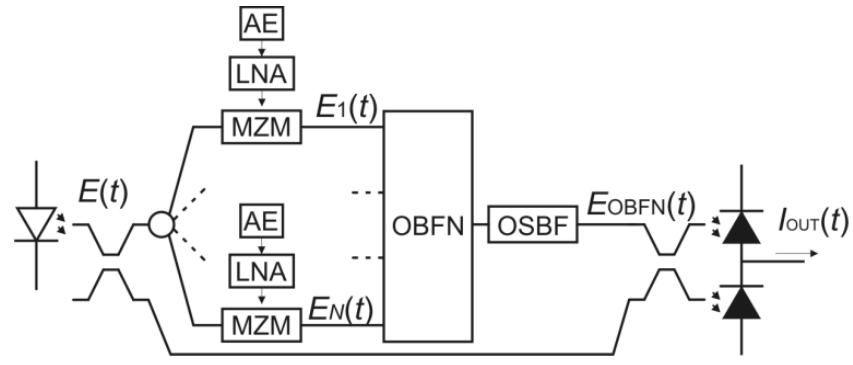

Figure 4.10: Photonic beam former system architecture in case of filter-based SSB-SC modulation and balanced coherent optical detection

functionality and performance, and therefore should be taken into account for the system design. Some major issues are discussed below:

\section{Polarization matching}

To combine optical signals, inside the OBFN and for carrier reinsertion, the polarizations of the combining optical signals should be kept the same to achieve optimal interference. This requires all the involved optical components to be polarization-maintaining, or else polarization controllers must be used. However, when the complete system is integrated on an optical chip, polarization matching between the optical signals can be well maintained.

\section{Optical phase stabilization}

As explained before, coherent combining of the optical signals requires optical phase synchronization, and the optical phases of the signals can be controlled by means of optical phase shifters. However, in practice those optical phases will fluctuate, especially when optical fibers are used in the system, resulting in power fluctuation in the system output or fluctuation of the beam direction. This is because the optical phase is very sensitive to the environmental changes in the signal path, such as changes in temperature, vibrations, and mechanical stress. Therefore, some measures are required for the optical phase stabilization in the beam former system. For one thing, it would be an easy task to achieve optical phase stabilization if all the optical components between the laser and the optical detectors, including modulators, were integrated on a single chip. Otherwise, when optical fibers are used in the system, a shielded setup surrounding the optical components can be used to reduce the external thermal and mechanical disturbances. Furthermore, inside the beam former system a possible solution for optical phase stabilization could be to use a frequency dithering scheme, as proposed within the framework of coherent optical code-division multiple access (OCDMA) techniques [25], but this has the disadvantage that it requires a unique oscillator for each beam former input, which 
considerably complicates the feeding circuit. Other than frequency dithering, a feedback loop from the system output back to the control of the OBFN phase synchronizers can also be used to achieve optical phase stabilization. This feedback mechanism could simply be based on maximizing the output signal power. However, this approach may have difficulty in achieving agile phase synchronization when the system has a large number of combining channels so that the relation between the output signal power and all the phase synchronizers is very complicated. Besides, this approach might not result in proper phase-locking when interferers from undesired beam directions are present.

\section{Imbalances in the optical detector}

In the earlier discussion, the carrier reinsertion coupler and the photodiode pair were assumed to be perfectly balanced, resulting in a theoretical output current Eq. (4.16) that is a perfect mixing product of the optical output signal of the OBFN and the unmodulated laser signal. In practice there will be slight imbalances, so that the individual intensities of those two terms will also appear in the output current. This will result in a residual DC level, residual IMD, and residual RIN. In general DC level will not be a problem, and the residual IMD and RIN will be very small compared to the desired mixing term. Likewise, the MZM will have some imbalance, resulting in residual optical carrier power at the output of the modulator, even when the MZM is biased at its minimum transmission point. This will also result in residual DC level and residual RIN in the output current. It can be shown that this will again be very small, as the carrier output power for commercial pushpull MZMs at the minimum transmission point will be well below 1 percent of the optical input power.

\subsection{Functional design of FLY beam former}

In this section the functional design of the beam former for the previously mentioned FLY project is described. The beam former is based on the advantageous architecture shown in Figure 4.10, including FDC, optical SSB-SC modulation and coherent optical detection.

\section{System requirements}

As described in Chapter 1, in the FLY project an 8-element linear array antenna is developed to receive signals from digital video broadcasting by satellite (DVB-S). The interelement distance of the FLY antenna is $1.18 \mathrm{~cm}$, and the desired beam scanning range is $\left[-60^{\circ}, 60^{\circ}\right]$. DVB-S signals operate in the $\mathrm{K}_{\mathrm{u}}$-band frequency range 10.7-12.75 GHz. After down conversion by low-noise block (LNB) the resulting IF signal for the OBFN is in the frequency range of 950-2150 MHz. Then, 
when this signal is modulated on an optical carrier, the bandwidth of an optical sideband is $1.2 \mathrm{GHz}$ and the frequency separation between the optical sidebands is $1.9 \mathrm{GHz}$. For the OBFN the DEs are required to provide a minimum bandwidth of $1.2 \mathrm{GHz}$ to cover one sideband of the optical signal. The maximum delay ripple should be kept in the order of tens of picoseconds, so that the corresponding phase error in the delayed signals is lower than $\pi / 16$, and therefore its effect on the antenna pattern is negligible [26], [27]. For the OSBF the bandwidths of the passband and stopband are required to be at least $1.2 \mathrm{GHz}$ and the bandwidth of the transition band is required to be less than $1.9 \mathrm{GHz}$ as shown in Figure 4.11.

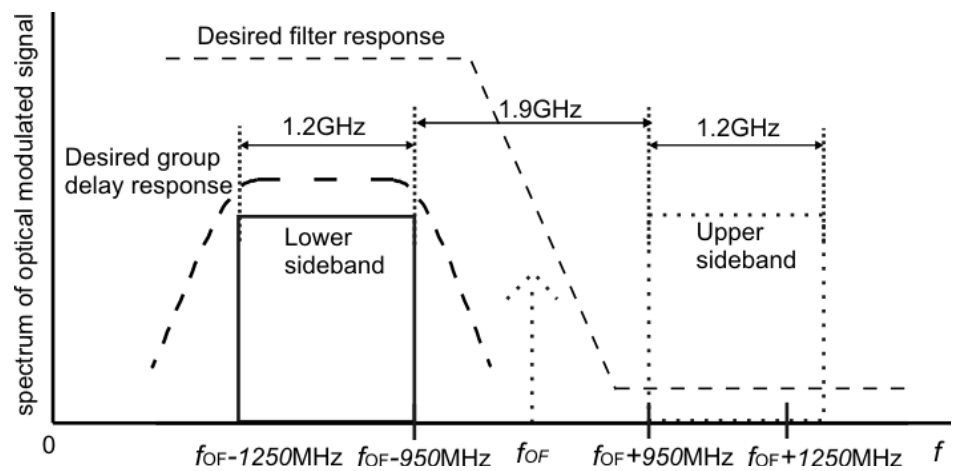

Figure 4.11: Bandwidth requirements for the DEs of OBFN and the OSBF for the FLY beam former system

\section{Design of OBFN}

The single-wavelength, asymmetrical binary-tree structured OBFN scheme is used for this application because of the advantages for both OBFN complexity and system cost as discussed in Chapter 3. For an 8-element antenna the OBFN is formed by a 3-stage binary tree. Based on Eq. (3.9) and the specifications of FLY antenna, the required maximum delay values for the 3 stages turn out to be 68,136 , and $272 \mathrm{ps}$, respectively. Then, using the ORR principles described in Section 2.4, it appears that one ORR is needed for each DE in the first and second stage; and two cascaded ORRs for the DE in the third stage in order to fulfill the delay requirements. The corresponding OBFN structure is shown in Figure 4.12. The complete OBFN consists of 8 ORRs, 7 optical combiners, and 7 optical phase synchronizers. In total 31 tuning elements are required in the OBFN. As explained in Chapter 3, $2 \times 2$ symmetric MZIs are used as the optical combiners in the OBFN. Thus, in practice one output of each combiner is used for the OBFN circuitry and if possible, the other one is made accessible from outside the chip, serving as a test port for checking the functionality of the connected OBFN section. 


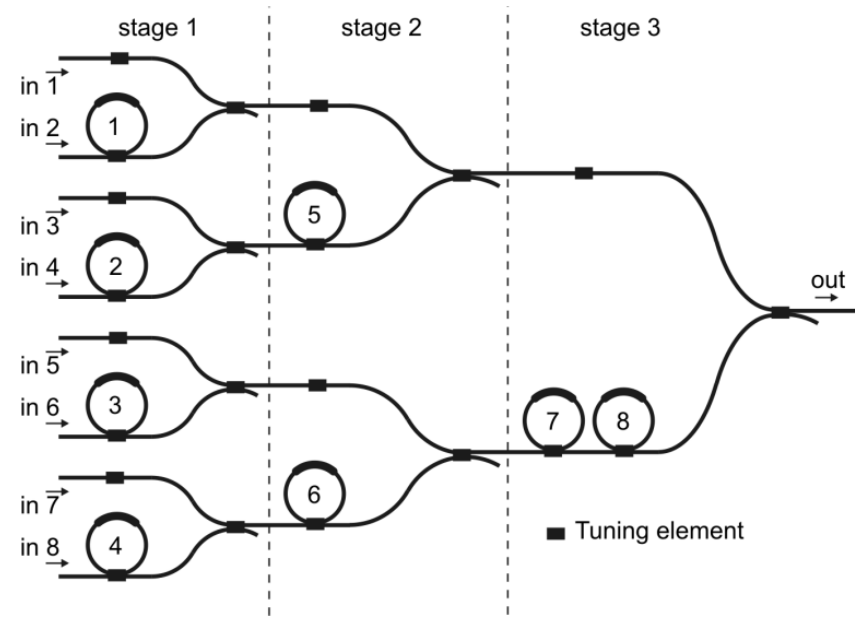

Figure 4.12: Structure of the OBFN for the FLY beam former

Design of $O S B F$

Besides the bandwidth descriptions of the OSBF given at the beginning of this section, the OSBF is also desired to have a flat passband and a broad stopband suppression. Based on the study on antenna patterns, the side lobes should be $25 \mathrm{~dB}$ lower than the main beam to keep the interference signals negligible, which correspondingly requires the OSBF stopband suppression to be at least $25 \mathrm{~dB}$. A preferable OSBF design scheme is to use a structure based on the same building blocks as in the OBFN, so that it would benefit system integration. Eventually, the OSBF structure chosen for this application is an asymmetrical MZI with an ORR in its shorter arm, which is shown in Figure 4.13. This OSBF requires a total of 5 tuning elements.

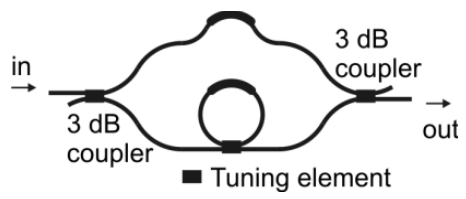

Figure 4.13: Structure of the OSBF for the FLY beam former

The roundtrip delay of the ORR $\tau_{r}$ should be twice as long as the path delay difference of the MZI $\tau_{d}$ (the corresponding FSR of the filter is $1 / \tau_{d}$ ). The advantages of such a filter are that it has flattened passbands and broadened stopbands with steep transitions in between, its phase response introduces negligible phase distortion to the signal [28], [29]. Based on the transfer matrix of the MZI given in Eq. (2.15), the transfer matrix of the OSBF can be given by 


$$
\boldsymbol{H}_{\mathrm{OSBF}}=\left[\begin{array}{cc}
c_{2} & -j s_{2} \\
-j s_{2} & c_{2}
\end{array}\right]\left[\begin{array}{cc}
H_{\mathrm{ORR}}(z) & 0 \\
0 & \sqrt{r} z^{-1} e^{-j \phi_{d}}
\end{array}\right]\left[\begin{array}{cc}
c_{1} & -j s_{1} \\
-j s_{1} & c_{1}
\end{array}\right]
$$

where $H_{\mathrm{ORR}}(z)$ represents the $z$-transform of the ORR in the OSBF (see Eq. (2.17)) and $\sqrt{r} z^{-1} e^{-j \phi_{d}}$ represents the $z$-transform of the longer arm of the MZI in the OSBF: $r$ is the roundtrip magnitude transmission of the ORR; $\phi_{d}$ is the additional phase shift of the MZI. Then, the $z$-transform of the OSBF shown in Figure 4.13 is given by

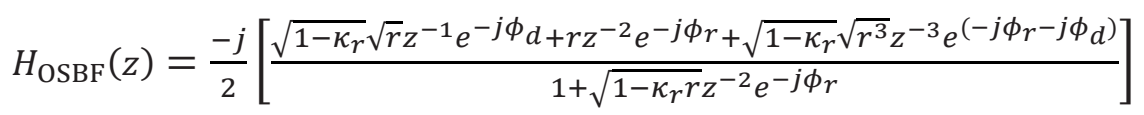

where $\kappa_{r}$ is the power coupling coefficient of the ORR. Assume such an OSBF is realized with negligible dimensions error and propagation losses $(r=1)$, the splitter and combiner of the MZI are realized with perfect $3 \mathrm{~dB}$ couplers, and the additional phase shift of the MZI $\phi_{d}$ is related to the additional roundtrip phase shift of the ORR $\phi_{r}$ by $\phi_{r}=2 \phi_{d}+\pi$, then the power transfer of the OSBF is given by [28]

$$
|H(f)|^{2}=\frac{\left[\sqrt{1-\kappa_{r}} \cos \left(3 \pi f \tau_{d}-\frac{3}{2} \phi_{d}\right)+\cos \left(\pi f \tau_{d}-\frac{1}{2} \phi_{d}\right)\right]^{2}}{2-\kappa_{r}+2 \sqrt{1-\kappa_{r}} \cos \left(4 \pi f \tau_{d}-2 \phi_{d}\right)}
$$

An illustration of one period of this power transfer is given in Figure 4.14. It shows the trade-off between the width and suppression of the stopband. The performance of this filter is independent of the FSR of the filter.

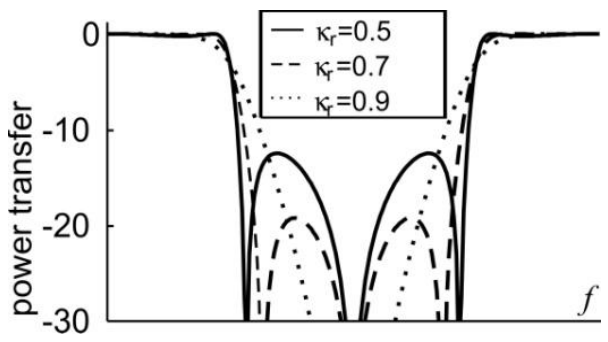

Figure 4.14: One period of the ideal power transfer function of the filter.

Now the FSR of the OSBF and the widths of the passband and stopband should be chosen such that one sideband of the modulated optical signal is passed (with sufficiently low passband ripple), and the other sideband is sufficiently suppressed. After calculation, an FSR of $6.7 \mathrm{GHz}$ (FSR of the asymmetric MZI is $6.7 \mathrm{GHz}$; FSR of the ORR is $3.35 \mathrm{GHz}$ ) and $\kappa_{r}=0.78$ are used for the OSBF. The corresponding simulation result is shown in Figure 4.15. 


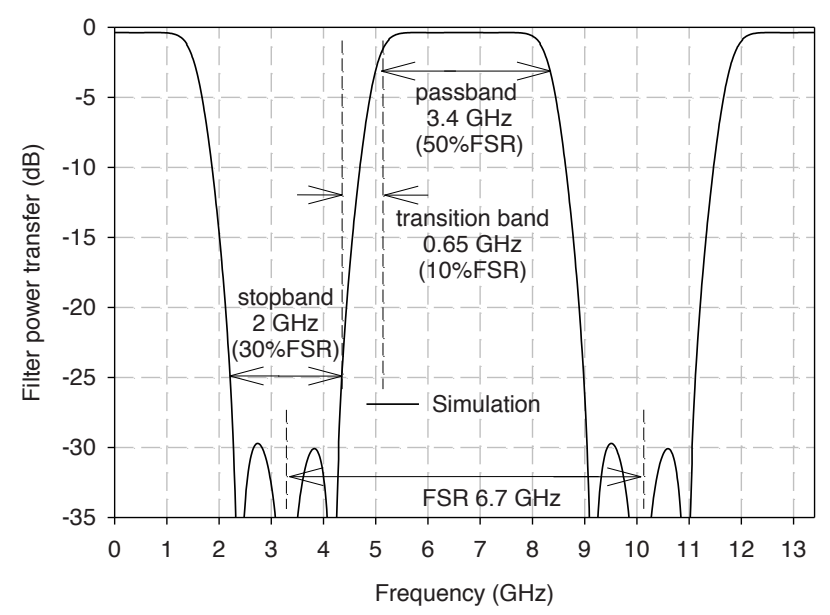

Figure 4.15: Theoretical power transfer of the OSBF

For the waveguide realization of the OSBF, it is likely that the roundtrip delay of the ORR $\tau_{r}$ is not exactly twice the delay of the longer arm of the MZI $\tau_{d}$ due to a small delay deviation $\tau_{e}$ in one of the delay paths. Figure 4.16 shows two numerical examples of the OSBF power transfer for the case $\tau_{r} / \tau_{d} \neq 2$. The effect of this mismatch between ORR and MZI appear to be that there is an additional periodic modulation on the power transfer of the OSBF in the frequency domain. The frequency period (FP) of this modulation is much larger than the FSR of the OSBF, roughly by a factor equal to the ratio between $\tau_{d}$ and $\tau_{e}$. However, as long as the delay deviation $\tau_{e}$ in the ORR or MZI is not significant (less than $10 \%$ of the designed delay value), one can still obtain one FSR where the OSBF power transfer is approximately the same as designed (in the middle of Figure 4.16 (a) and (b)).

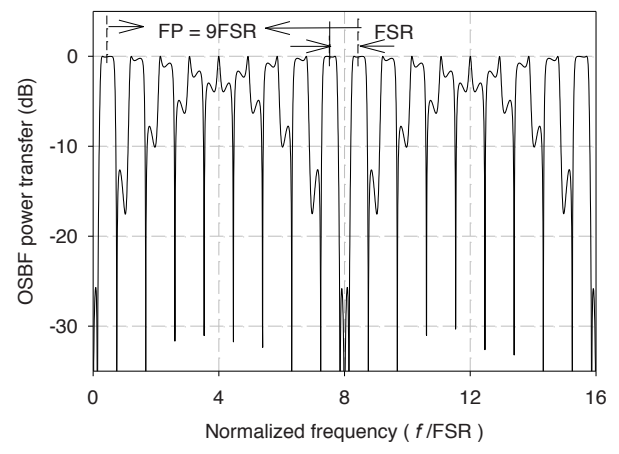

(a)

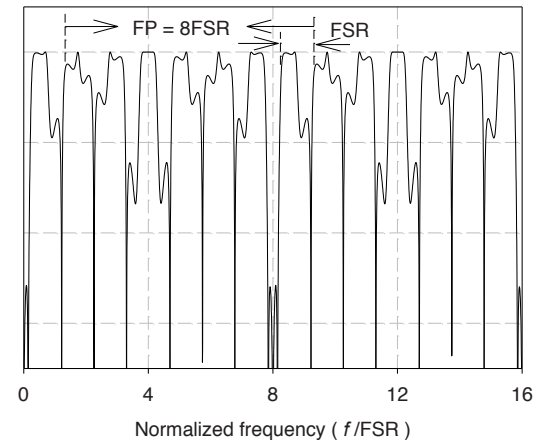

(b)

Figure 4.16: OSBF power transfers for (a) $\tau_{r} / \tau_{d}=2 / 1.125$ and (b) $\tau_{r} / \tau_{d}=2.125 / 1$. 
Design of the optical carrier reinsertion coupler

The optical carrier reinsertion coupler can be simply achieved by using a tunable $2 \times 2$ symmetric MZI-based coupler as those used in the OBFN. One input is connected to the output of the OSBF, whereas the other input and the two outputs should be accessible from outside of the chip, for optical carrier reinsertion and balanced detection, respectively.

\subsection{Functional design of SKY beam former}

\section{System requirements}

As described in Chapter 1, the SKY antenna is designed for radio astronomy application. It is a $4 \times 416$-element 2-D PAA. The antenna size is $50 \mathrm{~cm} \times 50 \mathrm{~cm}$ with the interelement distance of $16.67 \mathrm{~cm}$. Since the purpose of the SKY beam former is just to prove the concept of 2-D beam steering by means of the ORR-based OBFN, a small beam scanning range $\left[-5^{\circ}, 5^{\circ}\right]$ in both $\mathrm{XZ}$ and $\mathrm{YZ}$ plane is chosen, which leads to low requirement for the number of ORRs in the OBFN. The signal frequency range of this application is $400 \mathrm{MHz} 1600 \mathrm{MHz}$. Then, when this signal is modulated on an optical carrier, the bandwidth of one optical sideband is $1.2 \mathrm{GHz}$ and the frequency separation between the optical sidebands is $0.8 \mathrm{GHz}$. For the OBFN the DEs are required to provide a minimum bandwidth of $1.2 \mathrm{GHz}$ to cover one sideband of the optical signal. Based on the same principle as that for the FLY beam former, the maximum delay ripple should be kept in the order of tens of picoseconds, so that the corresponding phase error in the delayed signals is lower than $\pi / 16$, and therefore its effect on the antenna pattern is negligible [26], [27]. For the OSBF the bandwidths of the passband and stopband are required to be larger than $1.2 \mathrm{GHz}$ and the bandwidth of the transition band should be less than $0.8 \mathrm{GHz}$.

\section{Design of $O B F N$}

Similar to the FLY beam former, the single-wavelength, asymmetrical binary-tree structured OBFN scheme is used for the SKY beam former because of the advantages in both OBFN complexity and system cost. The SKY antenna consists of $16 \mathrm{AEs}$, therefore a $16 \times 1$ OBFN with 4 binary-combining stages is required. The layout of the OBFN and the mapping between the AEs and OBFN inputs are shown in Figure 4.17 (a) and (b), respectively. Figure 4.17 (a) shows that the section on the left-hand side combines the signals for every AE column (dashed boxes), corresponding to beam form in the angle plane parallel to the AE columns. Then, equivalently an $\mathrm{AE}$ row (dotted box) results after the column combination, and thus the section on the right-hand side is used to combine the signals of this AE row, 
corresponding to beam forming in the angle plane perpendicular to the AE columns. This way, the beam steering of the antenna can be performed in a 2-D manner. One ORR is used in each binary section of stage 1 for the combining in both vertical and horizontal directions, and a cascade of two ORRs are used in each binary section of stage 2 for the combining in both vertical and horizontal directions. The OBFN consists of 20 ORRs, 15 combiners, and 15 phase synchronizers. Correspondingly, 70 tuning elements are required in the OBFN.

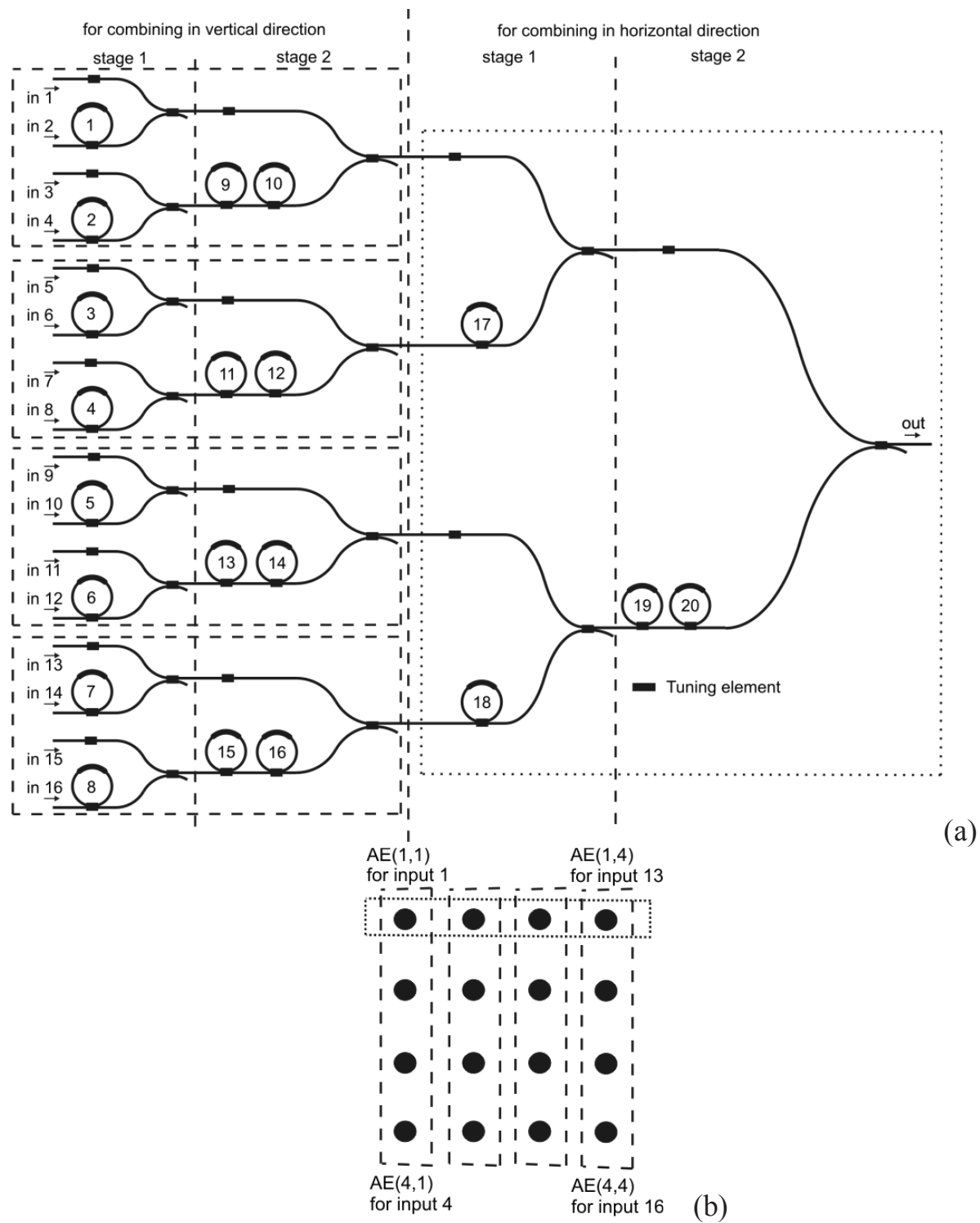

Figure 4.17: (a) Layout of $16 \times 1$ OBFN for a $4 \times 4$ PAA; (b) mapping between AEs and OBFN inputs. 
Design of OSBF and optical carrier reinsertion coupler

The OSBF and optical carrier reinsertion coupler of the SKY beam former use the same principle as that for the FLY beam former. However, the SKY OSBF is designed with an FSR of $4 \mathrm{GHz}$ to match the optical signal bandwidth characteristics.

\subsection{Conclusions}

After comparing different schemes for $\mathrm{E} / \mathrm{O}$ and $\mathrm{O} / \mathrm{E}$ conversions, a suitable architecture for photonic beam former systems was determined. This architecture includes FDC, filter-based optical SSB-SC modulation, and balanced coherent optical detection. It significantly relaxes the bandwidth requirements on the optical modulators, detectors, and OBFN. As a consequence, cheaper modulators and detectors with lower speed can be used in the system. For the OBFN lower bandwidth requirement means lower number of necessary ORRs and therefore lower OBFN complexity. Beside the advantage in the bandwidth requirement, this system architecture enhances the signal dynamic range of the beam former and also facilitates the system integration. Based on this architecture, the functional modules of beam former systems for FLY and SKY project are designed.

\section{References}

[1] H. Misawa, S. Juodkazis, A. Marchinkeicius, V. Mizeikis, A. Yamaguchi, H. Sun, S. Matsuo, "Laser microfabrication/manipulation of dielectric materials," Proceedings of 2000 International Symposium on Micromechatronics and Human Science, 22-25 Oct., 2000, pp 23-33.

[2] G. Motosuqi, Y. Yoshikuni, Y. Itaya, "Spectral characteristics of a DFB laser under high-speed direct modulation", Electronic Letters, vol.. 20, no. 21, Oct. 1984, pp 849-850.

[3] Y. -Y. Won et al., "OBI noise reduction using gain saturated SOA in reflective SOA based WDM/SCM-PON optical links", Electron. Lett., vol.. 42, no. 17, pp. 992-993, Aug. 2006.

[4] T. Minami, H. Nishimoto, M. Suyama, H. Kuwahara, T. Horimatsu, T. Touge, "Optical amplification and modulation techniques for multigigabit IM/DD long-haul transmission systems", vol. 1, SUPERCOMM/ICC '90 conference Record, 16-19 Apr. 1990, pp. 183-188.

[5] M. Stefanovic, A. Vidovic, "IM/DD optical system performance in the presence of dispersion, timing jitter and Gaussian noise," Proceeding of $5^{\text {th }}$ International Conference on Telecommunication in Modern Stellite, Cable and Broadcasting Service, vol.ume 2, 19-21 Sept. 2001, pp. 493-495. 
[6] S. Kaneko, M. Noda, Y. Miyazaki, H. Watanabe, K. Kasahara, T. Tajime, "An electroabsorption modulator module for digital and analog applications," $J$. Lightwave Technol., vol.. 17, no. 4, Apr. 1999, pp. 669-676.

[7] B. Masella, X. Zhang, "Linearized Optical Single-Sideband Mach-Zehnder Modulator for Radio-Over-Fiber Systems," IEEE Photonics Technology Letters, vol. 19, no. 24, Dec. 15, 2007, pp 2024-2026.

[8] K. K. Ng, Complete Guide to Semiconductor Devices, July 2002, $2^{\text {nd }}$ Edition, ISBN 0471202401.

[9] D. Mills, R. L. Allen, Signal Analysis, $1^{\text {st }}$ Edition, Jan. 2004, ISBN 0471234419.

[10] S. V. Kartalopoulos, Introduction to DWDM Technology, $1^{\text {st }}$ Edition, Dec. 1999, ISBN 0780353994.

[11] R. L. Freeman, "Telecommunication System Engineering," Wiley Seriers in Telecommunications and Signal Processing, June 2004, $4^{\text {th }}$ Edition, ISBN 0471451339.

[12] Dong-Pil Chang, In-Bok Yom, Seung-Pal Lee, "Development of a receiver downconvertor module for Ka-Band satellite payload," International Microwave Symposium Digest 2003, vol.. 3, 8-13 June 2003, pp. 1589-1592.

[13] A. Nirmalathas, G. H. Smith, D. Novak, "sensitivity analysis of optical SSB generation using a dual-electrode Mach-Zehnder modulator," Proceeding of International Topical Meeting on Microwave Photonics 1998, 12-14 Oct. 1998, pp. $79-82$.

[14] G. H. Smith, D.Novak, Z. Ahmed, "Techique for optical SSB generation to overcome dispersion penalties in fiber-radio sysems," Electron. Lett., vol. 33, no. 1, pp. 74-75, Jan. 1997.

[15] A. Molony, C. Edge, L. Bennion, "Fibre grating time delay element for phased array antennas," Electronics Letters, vol.. 31, no. 17, 17 Aug. 1995, pp. 14851486.

[16] S. B. Lowen, M. C. Teich, "Power-law shot noise," IEEE Transactions on Information Theory, vol.. 36, no. 6, Nov. 1990, pp. 1302-1318.

[17] R. F. Kalman, J. C. Fan, L. G. Kazovsky, "A novel analog optical link with high dynamic range," IEEE Photon. Technol. Lett., vol.. 5, no. 6, June 1993, pp. 725-728.

[18] T. K. Fong, M. Tabara, D. J. M. Sabido IX, L. G. Kazovsky, "Dynamic range of externally modulated analog optical links: optical amplification versus coherent detection," IEEE Photonics Technology Letters, vol.. 6, no. 2, Feb. 1994, pp. 270-272.

[19] M. Sieben, J. Conradi, D. Dodds, B. Davies, S. Walklin, "10 Gbit/s optical single sideband system," Electron. Lett., vol.. 33, no. 11, pp. 971-973, May 1997.

[20] S. Shimotsu, S. Oikawa, T. Saitou, N. Mitsugi, K. Kubodera, T. Kawanishi, M. Izutsu, "Single side-band modulation performance of a $\mathrm{LiNbO}_{3}$ Integrated 
modulator consisting of four-phase modulator waveguides, "IEEE Photon. Technol. Lett., vol.. 13, no. 4, pp. 364-366, Apr. 2001.

[21] M. Y. Frankel, R. D. Esman, "Optical single-sideband suppressed-carrier modulator for wide-band signal processing," J. Lightwave Technol., vol.. 16, no. 5, pp. 859-863, May 1998.

[22] A. Loayssa, C. Lim, A. Nirmalathas, D. Benito, "Design and performance of the bidirectional optical single-sideband modulator," J. Lightwave Technol., vol.. 21, no. 4, pp. 1071-1082, Apr. 2003.

[23] R. Montgomery, R. DeSalvo, "A novel technique for double sideband suppressed carrier modulation of optical fields," IEEE Photon. Technol. Lett., vol.. 7, no. 4, pp. 434-436, Apr. 1995.

[24] K. Oda, N. Takato, H. Toba, K. Nosu, "A wide-band guided-wave periodic multi/demultiplexer with ring resonator for optical FDM transmission systems," J. Lightwave Technol., vol.. 6, no. 6, pp. 1016-1023, June 1988.

[25] R. A. Griffin, D. D. sampson, D. A. Jackson, "Demonstration of datatransmission using cohereent coreelation to reconstruct a coded pulse sequence," IEEE Photon. Technol. Lett., vol.. 4, pp. 513-515, May 1992.

[26] A. Meijerink, C.G.H. Roelozffzen, R. Meijerink, L. Zhuang, D. A. I. Marpaung, M.J. Bentum, M. Burla, J. Verpoorte, P. Jorna, A. Hulzinga, and W. van Etten, "Novel Ring Resonator-Based Integrated Photonic Beamformer for Broadband Phased Array Receive Antennas-Part I: Design and Performance analysis," IEEE Journal of Lightwave Technology, vol.28, no. 1, pp. 3-18, January 2010.

[27] L. Zhuang, C.G.H. Roelozffzen, A. Meijerink, M. Burla, D. A. I. Marpaung, A. Leinse, M. Hoekman, R. Heideman, and W. van Etten, "Novel Ring ResonatorBased Integrated Photonic Beamformer for Broadband Phased Array Receive Antennas-Part II: Experimental prototype analysis," IEEE Journal of Lightwave Technology, vol 28, no. 1, pp. 19-31, January 2010.

[28] K. Oda, N. Takato, H. Toba, and K. Nosu, "A wide-band guided-wave periodic multi/demultiplexer with a ring resonator for optical FDM transmission system," J. Lightwave Technol., vol.. 6, no. 6, pp. 1016-1023, June 1988.

[29] C. K. Madsen and J. H. Zhao, Optical Filter Design and Analysis. New York: Wiley, 1999. 


\section{Chapter 5}

\section{Beam former chip realization}

\subsection{Introduction}

This chapter covers the design and fabrication of a beam former chip, which includes the optical beam forming network (OBFN), optical sideband filter (OSBF), and optical carrier reinsertion coupler for coherent optical detection. The chip is realized in a complementary metal-oxide-semiconductor (CMOS) compatible Lowpressure Chemical vapor deposition (LPCVD) planar waveguide technology $\left(\right.$ TriPleX ${ }^{\mathrm{TM}}$ waveguide technology $\left.{ }^{4}\right)$. First, the design of the elementary building blocks of the chip are described, namely the design of waveguide, power coupler, and tuning element. Next, the mask designs of the FLY and SKY beam former chip are presented. At the end of this chapter, the fabrication processes of the waveguides and heaters, as well as the implementation of the power coupling between fiber and chip are summarized.

\subsection{Waveguide design}

\subsubsection{Design requirements}

For the beam former chip realization, several waveguide properties are desired:

- Single mode waveguide

Due to the fact that different waveguide modes correspond to different propagation speeds, an ORR will introduce multiple delays when there are multiple waveguide modes present. Therefore, the single-waveguide-mode property is required for the beam former chip to make sure that for a certain chip setting an ORR-based delay element provides only one delay to the passing signals.

- Low insertion loss

The insertion loss of the chip consists of the coupling losses between fiber and waveguide, and the waveguide propagation loss, both of which should be as low as possible in order to maximize the optical power budget of the beam former.

\footnotetext{
${ }^{4}$ TriPleX ${ }^{\mathrm{TM}}$ waveguide technology is developed and patented by LioniX B.V. The Netherlands. Address: P. O. Box 456, 7500 AL, Enschede, The Netherlands. Phone: +31(0)53 489 3827, Fax: +31(0)53201 1303, E-mail: info@lionixbv.nl
} 
- Small bend radius

The bend radius of the waveguide should be as small as possible in order to minimize the required chip size. In the meantime, the bend loss (loss due to waveguide bending) should be such that it can be regarded as a negligible contribution to the waveguide propagation loss.

- Large group birefringence

For this beam former application, only transverse electric (TE) polarization is going to be used in order to avoid that TE and the transverse magnetic (TM) polarizations may result in different delays. Large group birefringence of the waveguide guarantees that the undesired coupling from TE to TM polarization, if there is any, is negligible.

\subsubsection{Waveguide characteristics}

For the beam former chip, silicon dioxide (silica) $\left(\mathrm{SiO}_{2}\right) /$ silicon nitride $\left(\mathrm{Si}_{3} \mathrm{~N}_{4}\right)$ waveguides are used. As the wave-guiding layer, $\mathrm{Si}_{3} \mathrm{~N}_{4}$ has large refractive index $(n=1.98$ at $1550 \mathrm{~nm})$ compared to $\mathrm{SiO}_{2}(n=1.46$ at $1550 \mathrm{~nm})$, enabling very small bend radius and hence compact chips [1]. However, $\mathrm{Si}_{3} \mathrm{~N}_{4}$ has a major drawback of large internal tensile stress, which limits its layer thickness to less than $350 \mathrm{~nm}$. As a consequence, $\mathrm{Si}_{3} \mathrm{~N}_{4}$ waveguides are usually designed with stripe geometries, which suffers relatively large PDL due to the poor confinement of TM-polarization. However, by combining $\mathrm{Si}_{3} \mathrm{~N}_{4}$ layers with layers of $\mathrm{SiO}_{2}$ which has large compressive stress, the total stress of the composite layer stack is significantly reduced. As a result, the thickness of the total stack can be considerably larger than the critical layer thickness of $\mathrm{Si}_{3} \mathrm{~N}_{4}$ alone [2]. This principle can also be used in the lateral direction, to further reduce the overall stress in the waveguide. This results in three different kinds of standardized single-mode waveguide geometries which could be used for the beam former chips, namely non-symmetrical, A-shaped, and box-shaped geometry (see Figure 5.1). In the latter two, the corresponding waveguide is formed by a shell of $\mathrm{Si}_{3} \mathrm{~N}_{4}$ filled with and encapsulated by $\mathrm{SiO}_{2}$. The schematics of these waveguide geometries and the corresponding scanning electron microscope (SEM) image of the realized waveguides are shown in Figure 5.1. The measured waveguide characteristics for these three different geometries are published in [3] and [4]. Here, they are given again in Table 5.1.

For the beam former chip, the box shape is preferred because of the fact that it does not have remaining slab waveguides. This avoids crosstalk between waveguide channels. Furthermore, since only TE polarization is required in this application and the coupling from TE to TM polarization should be as low as possible, the rectangular box shape is used for the waveguide geometry of the beam former chip 

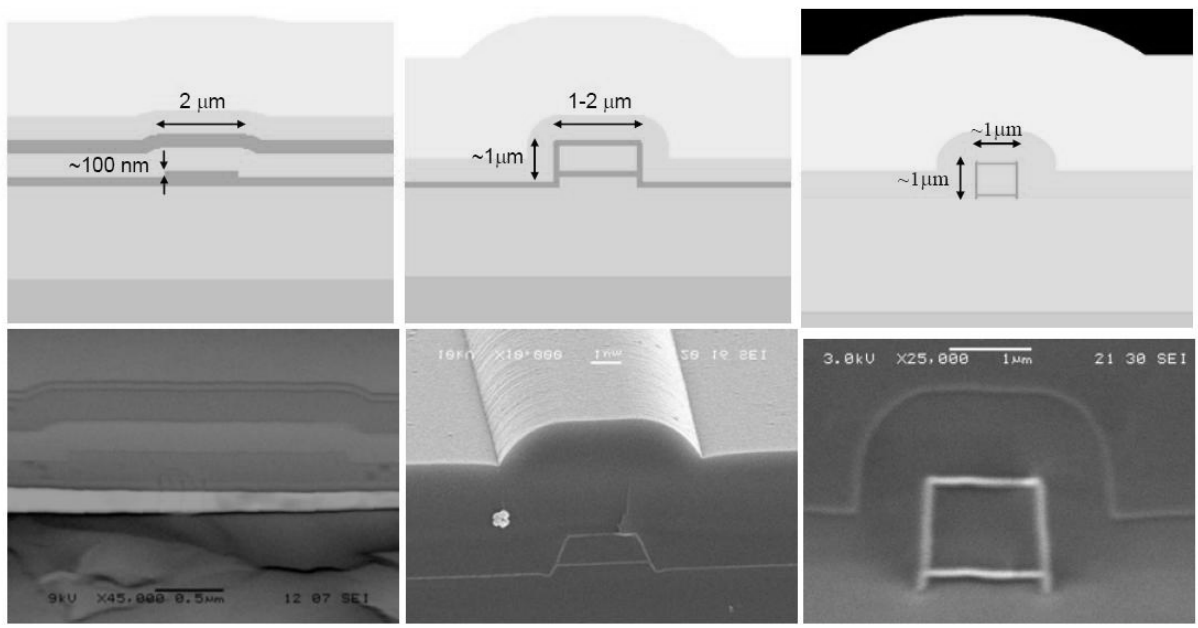

Figure 5.1: Schematics of non-symmetrical, A-shaped, and box-shaped waveguide geometries (top) and corresponding SEM image of the realized waveguides (bottom)

Table: 5.1: Measured characteristics of three waveguide geometries at $1550 \mathrm{~nm}$ [4]

\begin{tabular}{|l|c|c|c|}
\hline & $\begin{array}{c}\text { Non- } \\
\text { symmetrical }^{1}\end{array}$ & $\begin{array}{c}\text { A-shaped } \\
\text { geometry }^{1}\end{array}$ & $\begin{array}{c}\text { Box-shaped } \\
\text { geometry }^{2}\end{array}$ \\
\hline $\begin{array}{l}\text { Propagation loss in case of the } \\
\text { best waveguide sample }(\mathrm{dB} / \mathrm{cm})\end{array}$ & 0.12 & 0.10 & 0.06 \\
\hline $\begin{array}{l}\text { Insertion loss without spot size } \\
\text { converter }(\mathrm{dB})\end{array}$ & 8.0 & 1.4 & 0.6 \\
\hline Polarization dependent loss $(\mathrm{dB})^{4}$ & 0.20 & 0.12 & 0.38 \\
\hline Group birefringence & $\sim 1 \times 10^{-1}$ & $\ll 1 \times 10^{-3}$ & $\ll 1 \times 10^{-3}$ \\
\hline $\begin{array}{l}\text { Minimal bend radius }(\mu \mathrm{m})^{5} \\
\text { a bend loss } 7 \times 10^{-3} \mathrm{~dB} / \mathrm{cm}\end{array}$ & 400 & 750 & 500 \\
\hline
\end{tabular}

1: chip length $3 \mathrm{~cm}$

2: chip length $6.25 \mathrm{~cm}$

${ }^{3}:$ here, small core fibers were used (mode field diameter of $3.5 \mu \mathrm{m}$ )

${ }^{4}$ : it represents the ratio between the maximum and minimum transmission with respect to all possible states of polarization.

5: the value $7 \times 10^{-3} \mathrm{~dB} / \mathrm{cm}$ is used here for the measurement in order to guarantee that the possible fabrication deviation will not make the bend loss exceed $0.01 \mathrm{~dB} / \mathrm{cm}$ whose effect is considered to be negligible compared to the average waveguide propagation loss of $0.1 \mathrm{~dB} / \mathrm{cm}$. 
instead of the square box shape, and additionally different nitride layer thicknesses are used between top/bottom and sidewalls. The cross-section design of this waveguide and the corresponding SEM image of the realized waveguide are shown in Figure 5.2. The designed waveguide characteristics are given in Table 5.2.
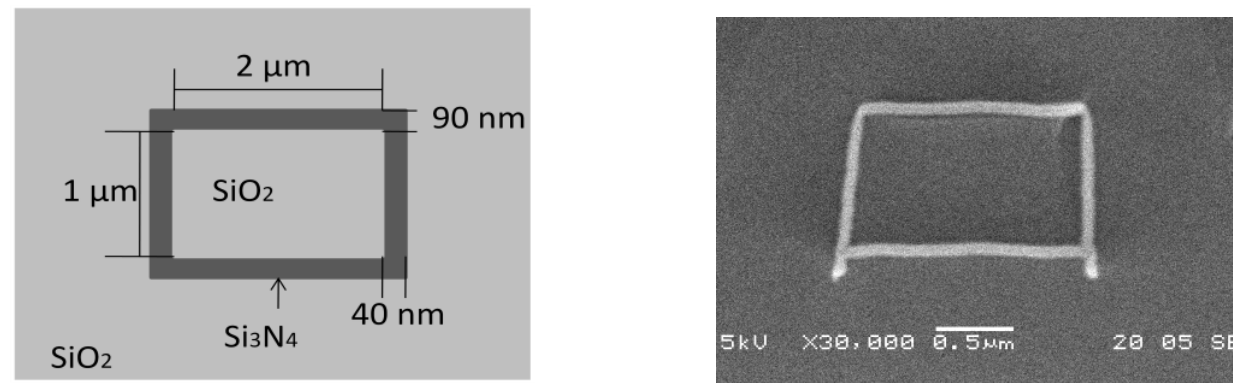

Figure 5.2: Schematics of rectangular box-shaped waveguide geometry for the beam former chip (left) and corresponding SEM image of the realized waveguide (right)

Table 5.2: Designed waveguide characteristics for beam former chips

\begin{tabular}{|l|c|}
\hline Waveguide mode & Single mode $(\mathrm{TE})$ \\
\hline Propagation loss $(\mathrm{dB} / \mathrm{cm})$ & $<0.1$ \\
\hline Fiber-chip coupling loss per facet $(\mathrm{dB})^{1}$ & 5 \\
\hline Bend radius $(\mu \mathrm{m})$ & 700 \\
\hline Bend loss $(\mathrm{dB} / \mathrm{cm})$ & $<0.01$ \\
\hline Effective index & 1.48 \\
\hline Group index & 1.51 \\
\hline
\end{tabular}

${ }^{1}$ Standard polarization maintaining fiber for $1550 \mathrm{~nm}$ is used

\subsection{Design of FLY and SKY beam former chips}

In chip realization, the waveguide geometry shown in Figure 5.2 is used to realize different systematic modules of the beam former chip (OBFN, OSBF, and optical carrier reinsertion coupler). In turn, these systematic modules are constructed out of several common elementary building blocks, such as optical phase shifters (OPS), power couplers, and feedback loops. The final mask layouts of the FLY and SKY beam former chips are determined by the designs of these elementary building blocks.

\subsubsection{Design of optical phase shifter}

As explained in Chapter 3, optical phase shifters are the basic tuning elements in the beam former system. For the beam former chip, a thermo-optic tuning mechanism is used for the tuning elements [5]. It provides a tuning speed in the order of sub- 
millisecond, which is much slower than its electro-optic counterpart [6], however, for beam former application it is fast enough. Besides, it is simple to implement, and it introduces very low extra loss in the waveguide. In comparison, the electro-optic material, such as liquid crystal, introduces higher attenuation [6], and the material discontinuity between the waveguide cladding and liquid crystal would cause light mode profile mismatch due to the difference in refractive index. Consequently, extra loss is introduced. Thermal-optic tuning is performed by means of heaters placed on top of a section of waveguide as shown in Figure 5.3, which is nothing else than a piece of metal with certain resistance. On the FLY and SKY beam former chips, the heaters and leads are made of chromium and gold, respectively. An example of heater circuitry layout is shown in Figure 5.4 (the arrows represent the flow of the heat).

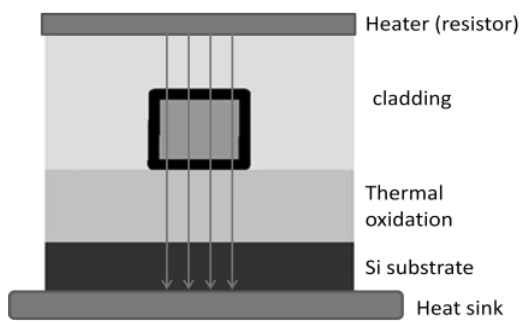

Figure 5.3: Cross-section of the OPS based on a thermo-optical tuning mechanism

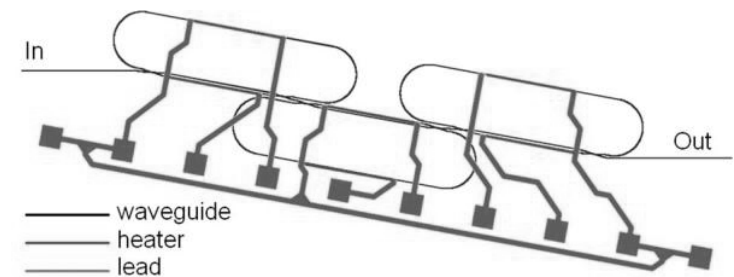

Figure 5.4: An example of heater circuitry layout

When a voltage is applied across the length of a heater, the electrical power is dissipated in the form of heat. This heat will cause a local temperature change, which consequently changes the effective index of the waveguide $N_{e}$. The change of $N_{e}$ over a section of waveguide will introduce an extra phase shift to the propagating light. It can be proved that this extra phase shift varies linearly with the dissipated power of the heater [7]. Based on simulation results, the heater with length of 2.2 $\mathrm{mm}$, width of $20 \mu \mathrm{m}$, and thickness of $69 \mathrm{~nm}$ has $800 \Omega$ resistance, and when $20 \mathrm{~V}$ is applied to it ( $0.5 \mathrm{~W}$ power dissipation), $2 \pi$ extra optical phase shift results, and in the meantime the heater temperature rises to around $100^{\circ} \mathrm{C}$ above room temperature, which remains far from the heater-damaging point (around $250{ }^{\circ} \mathrm{C}$ above room temperature). 


\subsubsection{Design of tunable optical power coupler}

For the beam former chip tunable $2 \times 2$ symmetric Mach-Zehnder interferometers (MZIs) are used as the power couplers of the ORRs, optical combiners in the OBFN, and the optical carrier reinsertion coupler. As explained in Chapter 2, such an MZI consists of two $3 \mathrm{~dB}$ couplers and an OPS section in-between. In practice directional couplers (DCs) are used as the $3 \mathrm{~dB}$-couplers, since DC introduces lower loss than its counterparts such as two-mode and multi-mode interferometers [8] and it is simply formed by two parallel waveguides which can be easily realized with the previously mentioned waveguide technology. Several MZI samples with different DC coupling lengths were measured on the MZI coupling tuning range, and the DC coupling length of $175 \mu \mathrm{m}$ results in the largest MZI coupling tuning range $\kappa_{\mathrm{MZI}}=[0, \ldots, 0.97]$. Using Eq. (2.12) the corresponding DC coupling coefficient $\kappa_{\mathrm{DC}}$ can be calculated (assuming the two DCs in the MZI have the same coupling coefficient) to verify the DC design. Based on the measured MZI coupling tuning range, the calculated $\kappa_{\mathrm{DC}}$ for different DC coupling lengths is shown in Figure 5.5. The DC coupling length of $175 \mu \mathrm{m}$ is chosen for the beam former chip. Besides, the designed DC has a waveguide gap of $2.5 \mu \mathrm{m}$ and a total length (including wavesplitting sections) of approximately $1 \mathrm{~mm}$. Based on this and the dimensions of the OPS heater, the corresponding MZI has a total length of approximately $5 \mathrm{~mm}$.

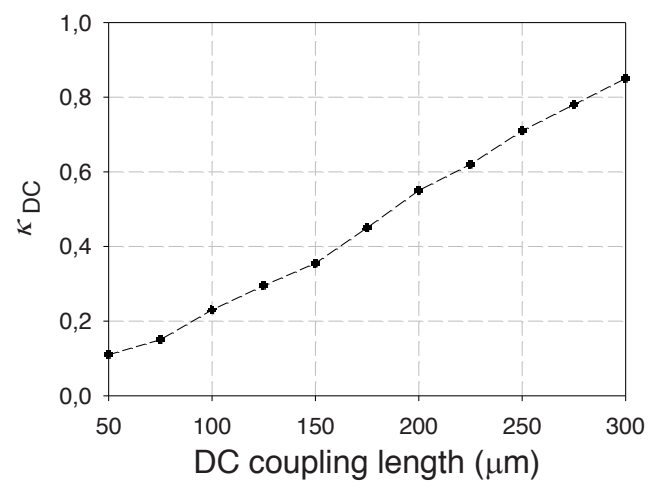

Figure 5.5: Calculated $\kappa_{\mathrm{DC}}$ for different DC coupling lengths

\subsubsection{Design of ORRs}

In order to save chip area, the ORRs are desired to be as small as possible. However, the size of an ORR is limited by the minimum bend radius of the waveguide (700 $\mu \mathrm{m})$ and the length of the MZI coupler $(5 \mathrm{~mm})$. Therefore, when the area-optimized race-track shape is used for the ORRs, the minimum ORR circumference appears to be $11.2 \mathrm{~mm}$. Using Eq. (2.3), the corresponding FSR of the ORR turns out to be 13.6 GHz around the wavelength of $1550 \mathrm{~nm}$. 


\subsubsection{Design of OSBFs}

For the OSBF, two tunable MZI couplers are used in the asymmetric MZI structure of the entire module. Again using Eq. (2.3), it can be calculated that for $6.7 \mathrm{GHz}$ FSR of the FLY OSBF the length difference between the two MZI arms is $28.9 \mathrm{~mm}$ and the circumference of the ORR is $57.8 \mathrm{~mm}$, whereas for $4 \mathrm{GHz}$ FSR of the SKY OSBF the length different between two MZI arms is $48.4 \mathrm{~mm}$ and the circumference of the ORR is $96.8 \mathrm{~mm}$.

\subsubsection{Design of chip mask layout}

When the designs of the elementary building blocks are finished, the next step is the design of the chip mask layout. In this step the software MaskEngineer ${ }^{5}$ is used. A geometrical requirement to the optical waveguide layout is that all branches from the inputs to the OBFN until the output of the OSBF have equal path length and equal optical losses. Although this is not very critical for correct system operation as the channel length can be adjusted in the RF path and the optical loss differences can be compensated by means of the optical combiners as explained in Chapter 3, it does significantly simplify the chip characterization.

To minimize the cost of manufacturing and maximize the manufacturing yield, the number of chips per wafer should be as large as possible. Therefore, the footprint of a chip should be minimized. Another general method used to increase the yield for such a complicated chip is the step-by-step approach: first the designed elementary building blocks are fabricated and characterized, and then the obtained empirical data is used to optimize the entire system design.

The last requirement on the mask design is that the input and output waveguides of the chip have a standard pitch of $250 \mu \mathrm{m}$, so that the chips can be pigtailed to commercially available fiber array units (FAUs). For the designed waveguide, polarization maintaining fibers (PMFs) are required for the coupling in order to guarantee the designed TE polarization of light, so that the maximum coupling efficiency can be achieved.

\section{- FLY beam former chip}

A photograph of the fabricated FLY chip and the mask set design are shown in Figure 5.6 (a) and (b), respectively. The chip size is $66.0 \mathrm{~mm} \times 12.8 \mathrm{~mm}$; five of these chips can be accommodated on a $10 \mathrm{~cm}$-diameter silicon wafer. Each chip has

\footnotetext{
${ }^{5}$ MaskEngineer is a commercial software product of PhoeniX B.V. The Netherlands. Adress: P. O. Box 545, 7500 AM, Enschede, The Netherlands. Phone: +31(0)53 483 6460, Fax : +31(0)53 4337415 , E-mail: info@phoenixbv.com
} 
39 heaters, 8 input waveguides and 10 output waveguides (system output and test ports), and underneath the lower row of bond pads there are some test structures of DCs and waveguides.

The optical waveguide layout was optimized for minimum chip footprint and for minimum optical path length differences and optical loss differences between the branches. The chip footprint was minimized firstly by flipping the rings of the ORRs, as shown in close-up in Figure 5.7, and secondly by folding the ring and MZI branches of the OSBF, as presented in close-up in Figure 5.8. The optical path length differences and optical loss differences between the branches were minimized firstly by making the design of the OBFN as symmetrical as possible. Secondly, the number of straight-to-bend transitions and the total angle of bent parts have been made equal for all channels in the OBFN and for the two pathways in the OSBF (both MZI branches and the ring). In the design process, optical crossing should be avoided as much as possible as they will introduce crosstalk between channels. Waveguide crossings are therefore only allowed between non-essential waveguides. The FLY beam former chip contains three of such waveguide crossings, marked with black circles in Figure 5.8. Additionally, in the center of the OBFN (see Figure 5.7) there are two waveguides that are not essential for the device operation, but could not be lead to the output waveguide section without crossing essential waveguides. Therefore, they are terminated by stray light absorbers, which are formed by a linear taper leading to a very small waveguide width, an 80 degreeangled waveguide end and a metal disc on top in order to attenuate the light leaking away. Because this waveguide-end angle is smaller than 90 degrees, the light that is still present after the stray light absorber will not result in additional interference due to Fabry-Perot resonances of substrate modes at the chip edges.

\section{- SKY beam former chip}

The SKY beam former chip is constructed with the same elementary building blocks as the FLY beam former chip, and the chip mask set design is also based on the same method. However, the SKY beam former chip contains 16 channels, 20 ORRs, and an OSBF with larger dimensions. Therefore, larger chip area is required to accommodate all those components, which corresponds to the chip size of $85 \mathrm{~mm} \times$ $25 \mathrm{~mm}$. With this chip size, only three chips can be accommodated on a $10 \mathrm{~cm}$ diameter silicon wafer. A photo of the fabricated SKY chip and the mask layout of the chip are shown in Figure 5.9 (a) and (b), respectively. The chip has 16 inputs, 10 outputs (system output and test ports), and 79 heaters. Figure 5.10 indicates the waveguide layout of the SKY beam former chip. 

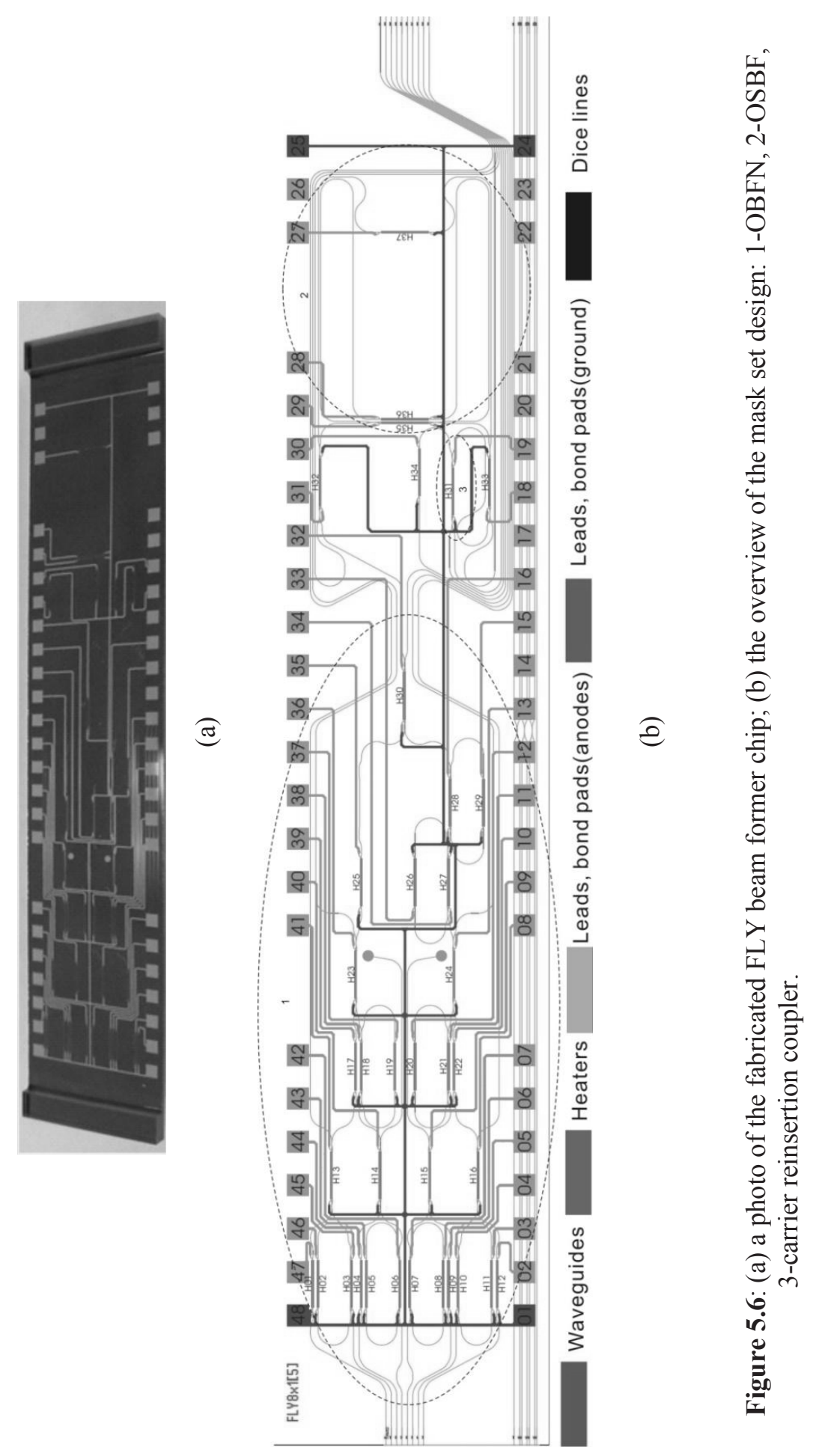


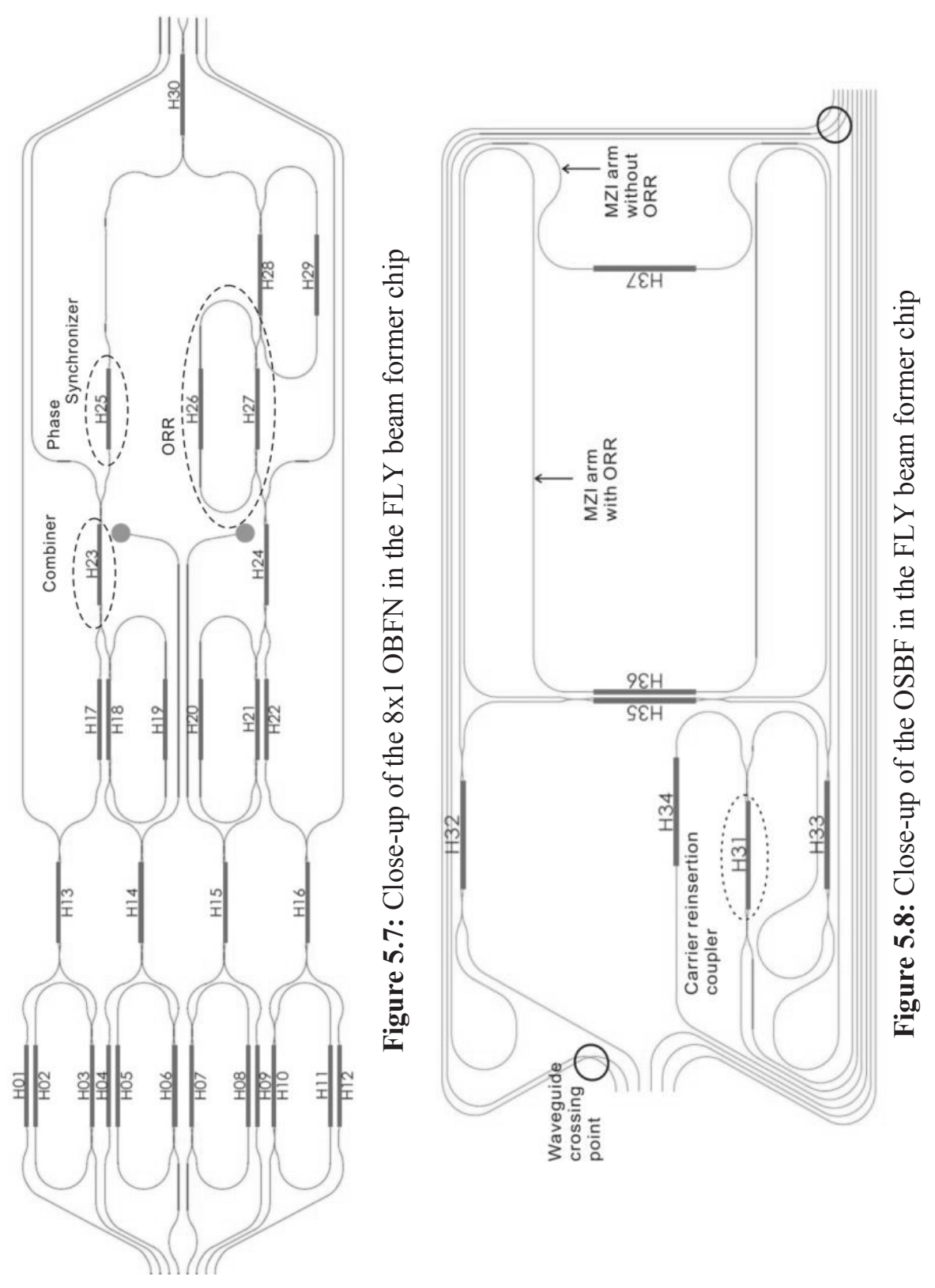



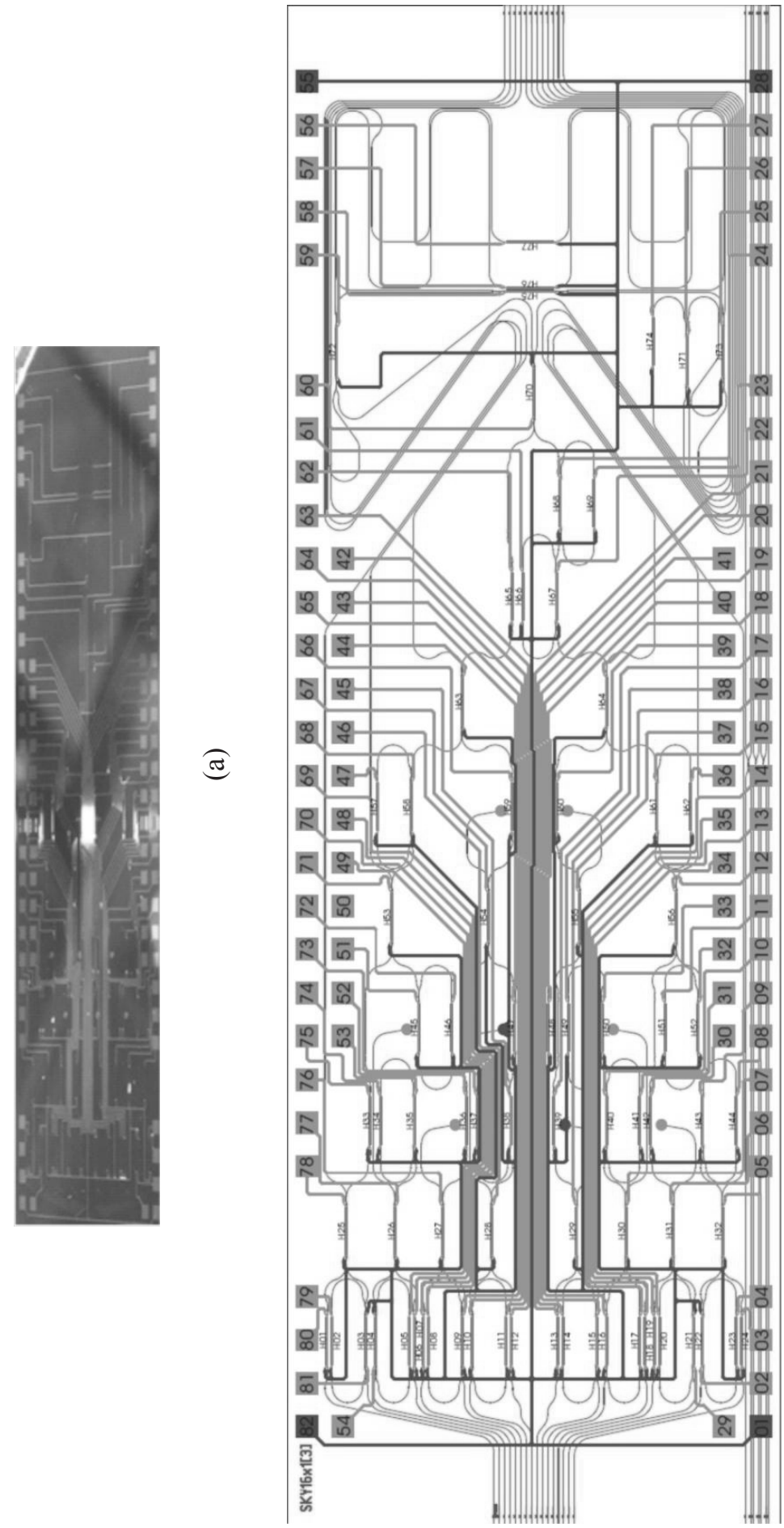

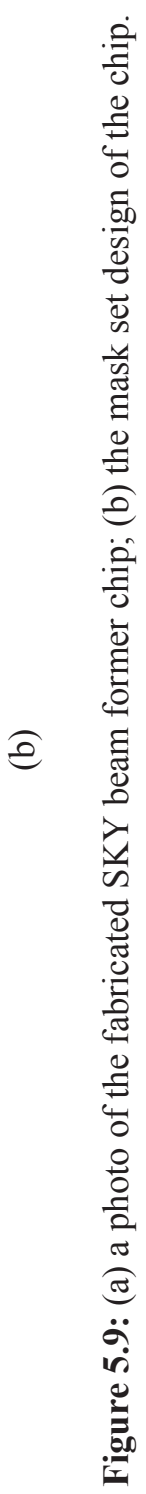




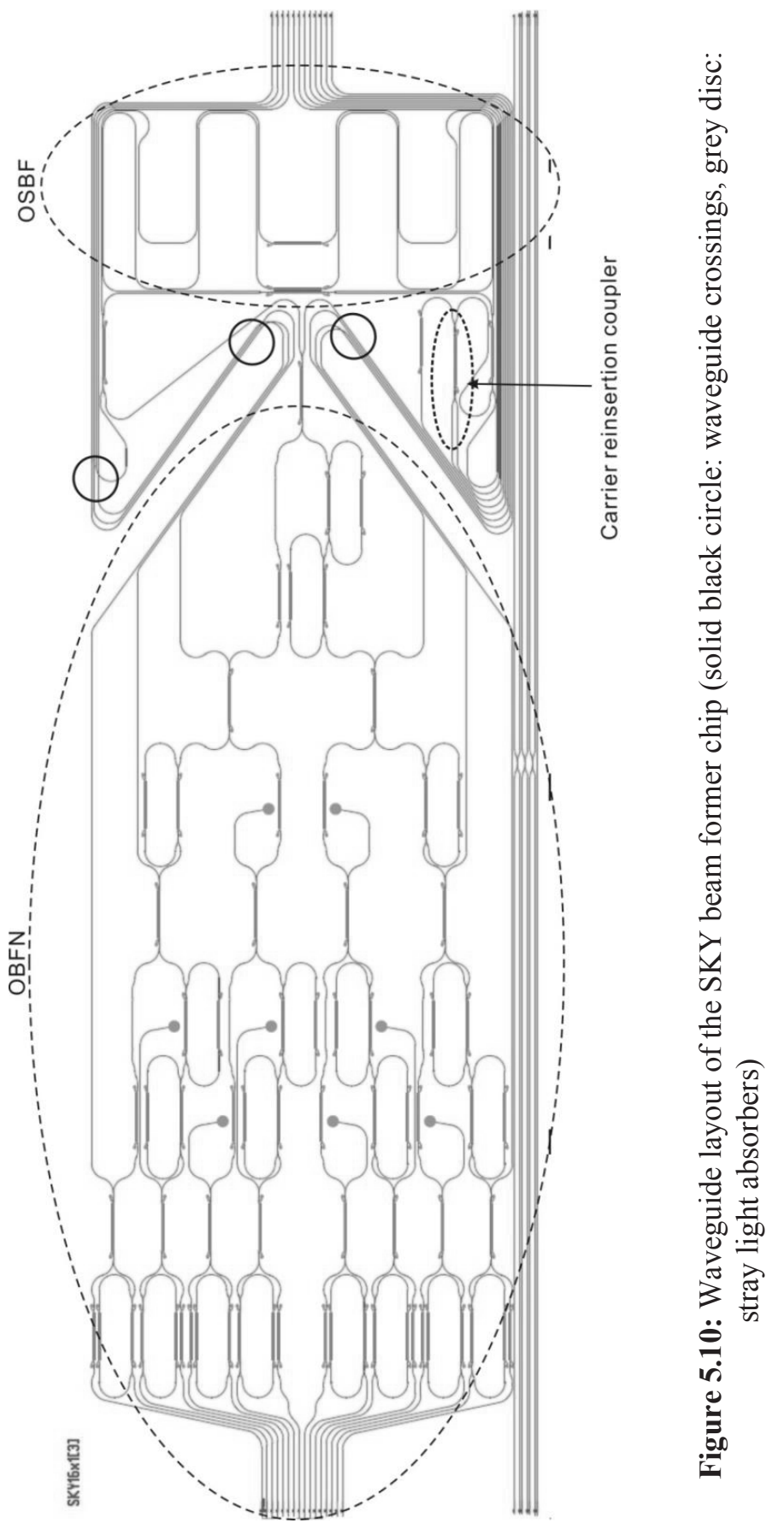




\subsection{Chip fabrication}

\subsubsection{Waveguide fabrication}

\section{- LPCVD process}

The applied waveguide technology uses the low pressure chemical vapor deposition (LPCVD) process to grow the required waveguide layers [9]. LPCVD is widely used for deposition of silicon oxide, nitride and poly-silicon layers. It enables relatively simple deposition of a layer of the required material, and the relatively slow deposition rate allows for precise control of the layer thickness and material characteristics. LPCVD processes at sub-atmospheric pressures, which tend to reduce unwanted gas-phase reactions and improve layer uniformity across the wafer. The deposition rate of LPCVD is mainly determined by the operation temperature and material. During the deposition process, fresh resource gas keeps filling in the reactor tube for the gas depletion by the reaction, however, the induction of fresh resource gas plays only a subordinate role for deposition rate. LPCVD allows many wafers to be processed at the same time, which can be arranged side by side in the gas flow. Therefore, LPCVD has significant advantage in production cost over other chemical vapor processes (such as plasma enhanced chemical vapor deposition, usually in short PECVD [10]). Besides, simple and reliable operation, uniform thickness of the product, and high reproducibility are also advantages of LPCVD. However, to guarantee a stable deposition process, LPCVD requires an operation temperature higher than $600^{\circ} \mathrm{C}$.

A schematic drawing of an LPCVD oven is shown in Figure 5.11. The base of the device is a quartz tube placed in a spiral heater. The operation steps are briefly described in Table 5.3.

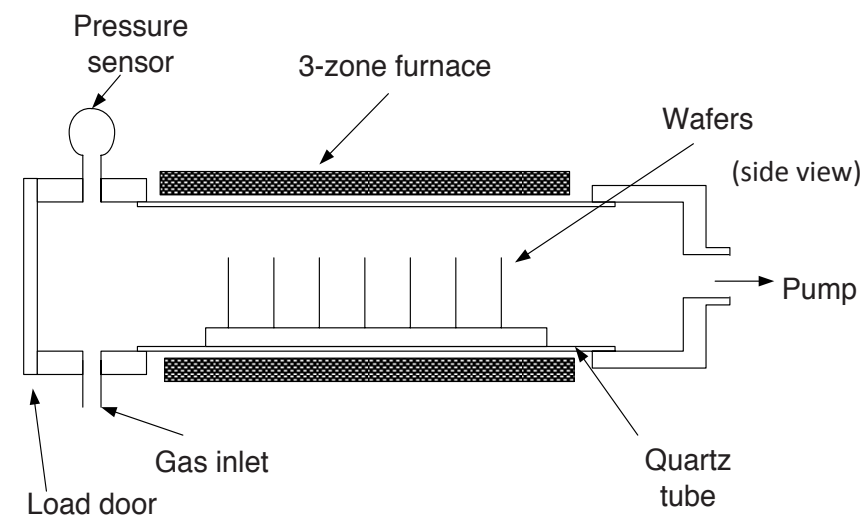

Figure 5.11: A schematic drawing of LPCVD oven 
Table 5.3: LPCVD operation steps

\begin{tabular}{|c|l|}
\hline Step & Process description \\
\hline 1 & Load reactor tube with an array of wafers placed in a quartz holder. \\
\hline 2 & $\begin{array}{l}\text { Evacuate the air in the tube to the pressure of } 0.1 \mathrm{~Pa} \text { and flush the tube } \\
\text { with pure nitrogen to remove the remaining oxygen. }\end{array}$ \\
\hline 3 & Heat up the tube to $800^{\circ} \mathrm{C}$ with temperature stability within $\pm 1^{\circ} \mathrm{C}$. \\
\hline 4 & $\begin{array}{l}\text { Enter resource gas in the tube. (Regularly, the resource gas consists of } \\
\text { reactive gas and gas for dilution, which spread inside the tube and flow } \\
\text { on the surfaces of the heated wafers. The operation pressure range is } \\
\text { from } 10 \text { to } 1000 \mathrm{~Pa} .)\end{array}$ \\
\hline
\end{tabular}

The factors that have influence on the thickness, uniformity and film content of the product are the wafer position, temperature profile in deposition zone, reactor geometry, deposition time, operation pressure, and the quantity and content of resource gases or vapors. In practice, all these factors need to be well controlled to guarantee the quality of the product.

\section{- Fabrication steps}

The applied waveguide technology involves one photolithography step only [11], which results in reduction of cost of fabrication and increase of yield. Although the details of the fabrication process are proprietary to LioniX B.V., the process is schematically described and illustrated in Table 5.4 and Figure 5.12, respectively.

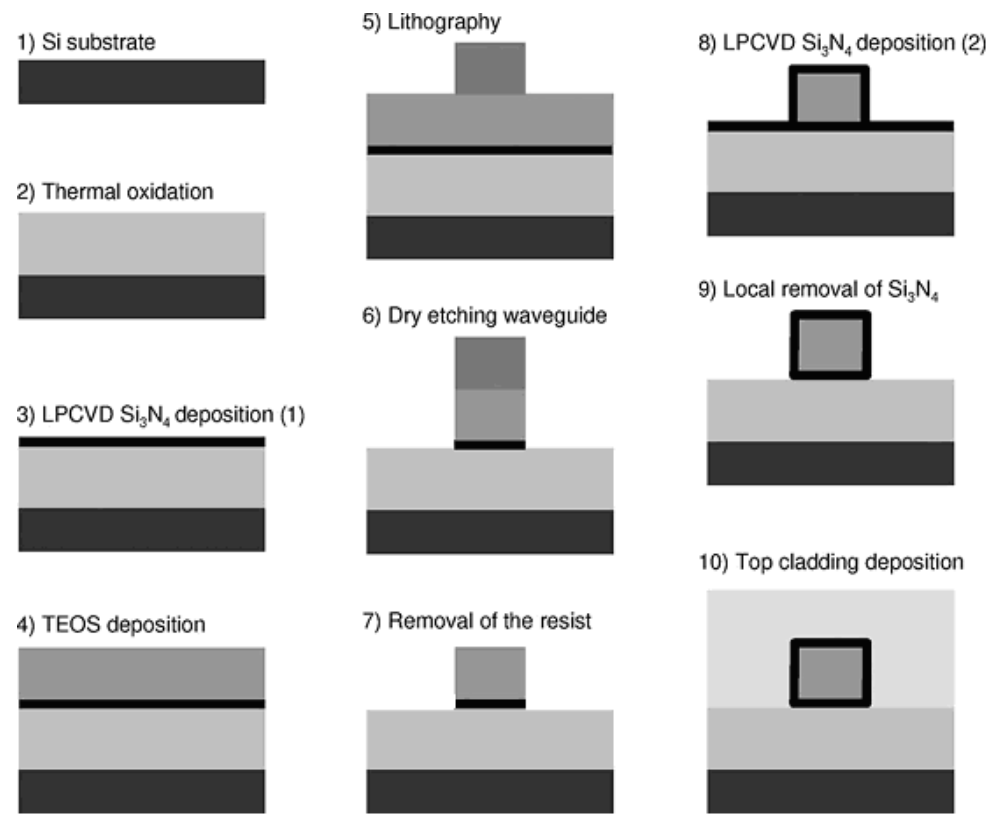

Figure 5.12: Schematics of the chip waveguide fabrication process 
Table 5.4: Schematical description of the chip waveguide fabrication process

\begin{tabular}{|c|c|}
\hline ep & \\
\hline 1 & -type, single-side polished, $100 \mathrm{~mm}$ diameter $\mathrm{Si}$ wafer for substrate. \\
\hline 2 & ing layer by thermal oxidation on $\mathrm{Si}$ wafer. \\
\hline 3 & $\begin{array}{l}\text { he } \mathrm{Si}_{3} \mathrm{~N}_{4} \text { bottom of the waveguide outer core with a thickness of } 90 \\
\text { PCVD. }\end{array}$ \\
\hline 4 & $\begin{array}{l}\mathrm{O}_{2} \text { layer of } 1 \mu \mathrm{m} \text { by means of Tetraethyl orthosilicate (TEOS)-to- } \\
\text { mical conversion. }\end{array}$ \\
\hline 5 & $\begin{array}{l}\text { graphy to obtain image printing of the waveguide } \\
\text { yer. }\end{array}$ \\
\hline 6 & tching (dry etching) process to achieve \\
\hline 7 & $\begin{array}{l}\text { he dry etching stops at the lower cladding layer. Then the remaining } \\
\text { hotoresist is removed. }\end{array}$ \\
\hline 8 & $\begin{array}{l}\text { eate the } \mathrm{Si}_{3} \mathrm{~N}_{4} \text { top and sidewall of the waveguide outer core with } \\
\text { ckness of } 90 \mathrm{~nm} \text { and } 40 \mathrm{~nm} \text { by LPCVD, respectively. }\end{array}$ \\
\hline 9 & haped geometry. \\
\hline 10 & $\begin{array}{l}\text { Deposit and passivate the top cladding layer, which has the thickness of } \\
\geq 5 \mu \mathrm{m} \text {. In this step, a combination of TEOS deposition }(1 \mu \mathrm{m}) \text { and } \\
\mathrm{PECVD} \mathrm{SiO} 2(\geq 4 \mu \mathrm{m}) \text { is used to speed up the process. At the end of the } \\
\text { fabrication process, the whole layer stack is annealed at } 1150^{\circ} \mathrm{C} \text { to reach its } \\
\text { final stable characteristics. }\end{array}$ \\
\hline
\end{tabular}

\subsubsection{Heater fabrication}

After the waveguide fabrication, the heaters of the beam former chip are fabricated by placing chromium and gold on the chip. The heater fabrication process is described and illustrated in Table 5.5 and Figure 5.13, respectively.

Table 5.5: Descriptions of the heater fabrication process for the beam former chip.

\begin{tabular}{|c|l|}
\hline Step & Process description \\
\hline 1 & Coat the chip with a photoresist layer. \\
\hline 2 & Perform lithography according to heater positions. \\
\hline 3 & Create a $\sim 70 \mathrm{~nm}$-thick chromium layer by means of sputtering. \\
\hline 4 & $\begin{array}{l}\text { Remove the remaining photoresist together with the chromium on top of it } \\
\text { by means of the lift-off process. }\end{array}$ \\
\hline 5 & Coat the chip with a photoresist layer again. \\
\hline 6 & $\begin{array}{l}\text { Perform lithography again with a second mask according to the lead } \\
\text { circuitry layout. }\end{array}$ \\
\hline 7 & Create a 1-2 $\mu \mathrm{m}$-thick gold layer by means of sputtering. \\
\hline 8 & Remove the remaining photoresist by means of the lift-off process. \\
\hline
\end{tabular}




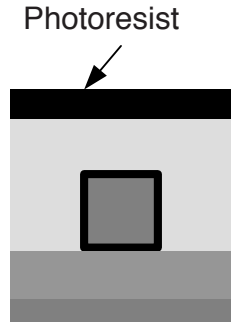

(1)

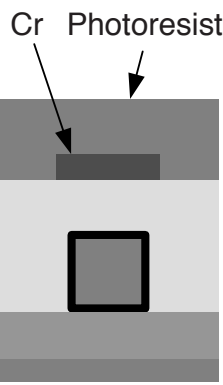

(5)

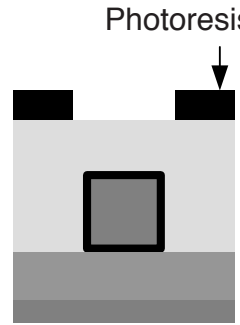

(2)

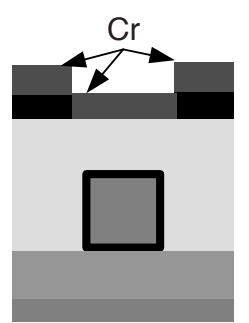

(3)

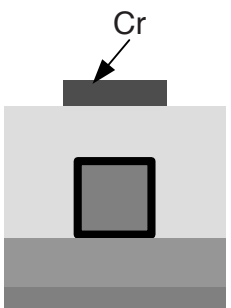

(4)

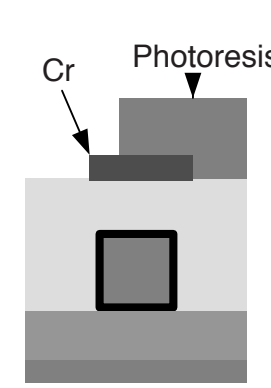

(6)

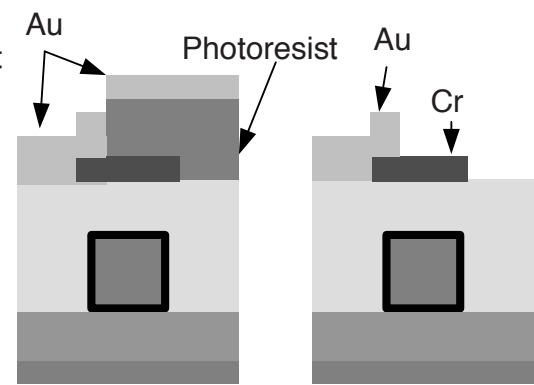

(8)

Figure 5.13: Illustration of the heater fabrication process for the beam former chip

\subsection{Coupling between fibers and chip}

Another important issue for the chip realization is the fiber-chip coupling. High efficiency, low cost, and good reliability are required for the coupling implementation. For the beam former chip, the techniques with butt-coupling and active alignment are used.

Active alignment can be regarded as a close-loop control process, in which the system parameters such as coupled light power are monitored and fed back to a controller that adapts the alignment state accordingly [12]. This way, the maximum power coupling can be achieved. Using active alignment means that there is no need for processing steps in the chip fabrication such as the making of on-chip alignmentgrooves. This is an advantage for the research on the chip fabrication since the fabrication process itself is not standard and can be changed. Active alignment is applicable for simultaneous alignment of multiple fibers. In this case the fiber array units (FAUs) are required. If the spacing between the fibers matches to the waveguide facets, the alignment of the two outer fibers suffices for aligning the entire fiber array. Furthermore, polarization maintaining fibers (PMFs) are required 
due to the polarization-dependency of the waveguide (TE polarization/electrical field in horizontal direction). A scanning electron microscope (SEM) image of PMFs on the dedicated V-groove array is shown in Figure 5.14.

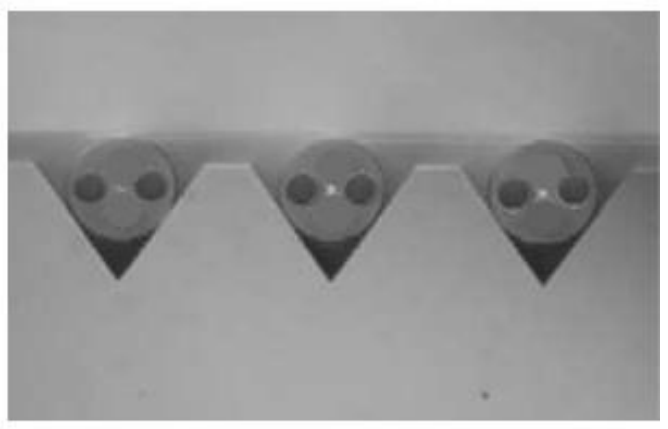

Figure 5.14: SEM image of PMFs in a V-groove array for the beam former chip

In our application butt-coupling is more preferable than the other coupling techniques such as prism-coupling and grating-coupling [13] because of the simple implementation and good coupling efficiency, and also the fact that the optical chip can easily be sawn or cleaved and if necessary be polished. After performing the butt-coupling and active alignment, the FAUs (PMFs) are glued to the beam former chip, as shown in Figure 5.15 (the chip is placed on top of a copper base, and its heater bondpads are reached via metal pins). Since there is only a very thin layer of glue between FAUs and the chip, the loss caused by the glue can be neglected. A coupling loss of $5 \mathrm{~dB} /$ facet is observed from measurements, which matches the designed value shown in Table 5.2. This large coupling loss is caused by the mismatch in mode field diameter (MFD): the PMF has a modal profile with an MFD of approximately 10 microns, and the fabricated waveguide has an MFD of approximately 3 microns. However, this coupling loss can be significantly lowered by tapering the nitride layers in the waveguide and in that way increasing the modal profile. Then, the corresponding coupling loss is expected to be in the order of 0.5 $\mathrm{dB} /$ facet. Although this process is currently being fine tuned at LioniX (with very promising results), the aim of this beam former chip realization is to practically verify the beam former concepts explained in the previous chapters instead of pursuing the best chip performances. Therefore, the waveguide tapering is skipped in the current waveguide fabrication process to avoid possible yield issues. An even better solution for future implementation would be to completely avoid fiber pigtailing and directly integrate the light sources via another packaging technology such as flip chipping of Vertical-Cavity Surface-Emitting Laser (VCSELS) [14], [15]. 


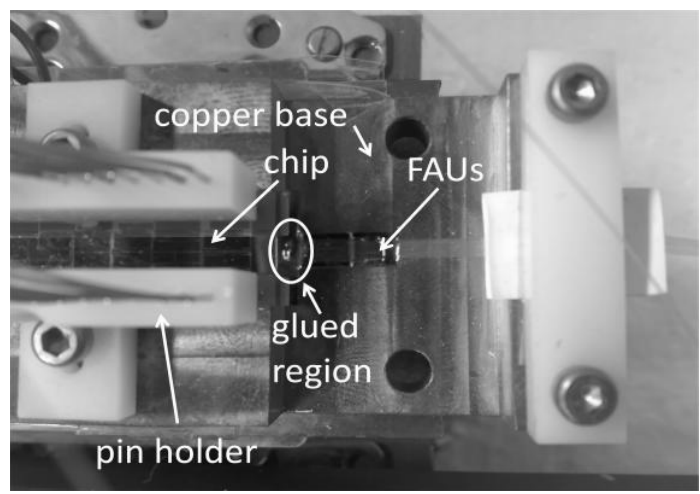

Figure 5.15: Picture of the FAUs pigtailed to the beam former chip

\subsection{Conclusion}

The beam former chip includes OBFN, OSBF, and the optical carrier reinsertion coupler. The applied waveguide technology allows propagation loss less than 0.1 $\mathrm{dB} / \mathrm{cm}$ and minimum bend radius of $700 \mu \mathrm{m}$ for bend loss up to $7 \times 10^{-3} \mathrm{~dB} / \mathrm{cm}$, which benefits the chip quality and compactness. Since the waveguide fabrication is based on LPCVD process and requires only one photolithography step, low fabrication cost and simple implementation are achieved. Thermo-optical tuning mechanism is chosen for the beam former chip, which provides sufficient tuning speed, introduces very low extra loss in the waveguide, and brings no implementation difficulty. Chromium and gold are used for the heaters and leads, respectively. Active alignment and butt-coupling technique are used for the coupling between the chip and fibers, which are easy to implement, independent of waveguide fabrication process, and reliable for achieving maximum power transfer. Moreover, glue is used for the pigtailing of PMF FAUs to the beam former chips, which is characterized by good stability and negligible loss.

\section{References}

[1] S. Zheng, H. Chen, and A. W. Poon, "Microring-resonator cross-connect filters in silicon nitride: Rib waveguide dimensions dependence," IEEE J. Sel. Topics Quantum Electron., vol. 12, no. 6, pt. 2, pp. 1380-1387, Nov./Dec. 2006.

[2] "Low modal birefringent waveguides and methods of fabrication", U. S. patent application nr. 10/756627-001, Jan. 2004.

[3] R. Heideman, A. Melloni, M. Hoekman, A. Borreman, A. Leinse and F. Morichetti, "Low loss, high conrast optical waveguides based on CMOS 
compatible LPCVD processing: technology and experimental results", Proceedings IEEE/LEOS Symposium Benelux Chapter, p.71-74, Dec. 2005.

[4] R. Heideman, A. Leinse, W. Hoving, R. Dekker, D. Geuzebroek, E. Klein, R. Stoffer, C. Roeloffzen, L. Zhuang, A. Meijerink, "Large-scale integrated optics using TriPleX ${ }^{\mathrm{TM}}$ waveguide technology: from UV to IR", SPIE Photonics West, San Jose, California, 24-29 Jan. 2009, pp. 7221-7226.

[5] Se-Young Seo, Jinku Lee, Jung H. Shin, Eun-Seok Kang and Byeong-Soo Bae, "The thermo-optic effect of Si nanocrystals in silicon-rich silicon oxide thin films", Applied Physics Letters, vol. 85, no. 13, Sep. 27, 2004, pp. 25262528.

[6] Ikushima Akira, "Functional Glass Material. Electro-Optic Effect", J. Optical and Electro-Optical Engineering Contact, vol.37, no.12, pp.794-800, 1999.

[7] Chris Roeloffzen, Passband flattened binary- tree structured add-drop multiplexers using SiON waveguide technology, PhD thesis (ISBN 90-3651803-2), University of Twente, Enschede, The Netherlands, 2002

[8] Ivan P. Kaminow; Tingye Li, Optical fiber telecommunications IV Volume A: Components, Academic Press, San Diego, 2002

[9] Hao Jin and K.J. Weber, "The effect of LPCVD silicon nitride deposition on the $\mathrm{Si}-\mathrm{SiO} 2$ interface of oxidised silicon wafers" J. Electrochemical Society, vol. 154, no. 1, H5-H8, 2007.

[10] A. Stoffel, A. Kovacs, W. Kronast and B. Muller, "LPCVD against PECVD for micromechanial applications ", J. Micromech. Microeng, vol.2 pp. 1-13, June 1996.

[11] Richard C. Jaeger, "Lithography": Introduction to Microelectronic Fabrication. Upper Saddle River: Prentice Hall. ISBN 0-201-44494-7, 2002.

[12] Ivan P. Kaminow; Tingye Li, Optical fiber telecommunications IV Volume B: Systems and impairments, Academic Press, San Diego, 2002.

[13] Robert G. Hunsperger , Integrated optics: theory and technology, Springer, 2002.

[14] T. Ouchi, A. Imada , "Direct coupling of VCSELs to plastic optical fibers using guide holes patterned in a thin photoresist". IEEE Photonics Technol. Lett., vol. 14, no. 3, pp. 263-265, 2002.

[15] Murata Yoshikazu, Miyazato Kentaro, Watanabe Noritoshi, Mikami Osamu, Ichimura Akira, and Hiramatsu Seiki, "Optical Coupling between VCSEL and Optical Fiber with 45-degree Micro Mirror", J. IEIC Technical Report (Institute of Electronics, Information and Communication Engineers), ISSN:0913-5685, vol.102, no. 1, pp.15-18, 2002. 


\section{Chapter 6}

\section{Control system for the beam former chip}

\subsection{Introduction}

As described in the previous chapter, the beam former chip is controlled thermaloptically, and the chip tuning is performed by means of resistor-based heaters. Therefore, a dedicated heater controller is required for the tuning purpose. In addition to that, a chip temperature control setup is also needed in the system to prevent the deviation of tuning parameters caused by the temperature change of the chip. Beside the hardware for the control system, an automatic tuning algorithm is required to translate the desired beam angle of the antenna into the voltages supplied to the heaters. In this chapter, the control system for the beam former chip is described.

\subsection{Heater controller}

\subsubsection{Design requirements}

The heater controller is supposed to supply voltages to all the heaters on the chip at the same time. Therefore, the heater controller must have as many voltage outputs as the heaters, namely 38 outputs for the FLY chip and 79 outputs for the SKY chip (as shown in Chapter 5). To achieve the complete tuning ability of the chip, the maximum output voltage of the heater controller must guarantee that the heaters (optical phase shifters) in the chip can generate additional optical phase shifts in the full tuning range $[0, \ldots, 2 \pi]$ as explained in Chapter 3 . In Chapter 5 it is mentioned that the heaters are designed to generate $2 \pi$ additional optical phase shift at $20 \mathrm{~V}$. However, the properties of the fabricated heaters may have small deviations from the designed values. Therefore, the maximum output voltage of the heater controller is set to be $30 \mathrm{~V}$ to guarantee that all the heaters can reach their full tuning range. It is explained in Chapter 3 that the beam angle can be expressed as a function of the voltages on the heaters. Therefore, the minimum voltage tuning step of the heater controller determines the minimum beam steering step. Based on Example 3.9 and the antenna specifications described in Chapter 4, it can be deduced that when the minimum voltage tuning step of the heater controller is $10 \mathrm{mV}$, the required minimum beam steering step of lower than 1 degree can be achieved for both FLY 
and SKY antennas. Furthermore, to reduce the chance of over-current damage in the ground line of the heater circuitry, the heater controller should operate in such a way that half of the heaters are supplied with positive voltages and the other half negative voltages. It is preferred that the heater controller is able to operate both independently and together with a PC. Therefore, a microcontroller is needed for the heater controller, which is able to calculate the heater voltages according to the desired beam angle of the PAA and communicate with a PC. Besides, data storerecall ability is also required for the heater controller to save and load fixed heater voltage settings. Table 6.1 gives an overview of the design requirements of the heater controller.

Table 6.1: Heater controller design requirements

\begin{tabular}{|l|l|}
\hline No. of outputs & 38 outputs for the FLY chip; 79 outputs for SKY chip. \\
\hline Maximum output voltage & $30 \mathrm{~V}$ \\
\hline Maximum current & $37.5 \mathrm{~mA}$ (for the designed heater resistance of $800 \Omega$ ) \\
\hline Maximum power & $1.1 \mathrm{~W}$ (for the designed heater resistance of $800 \Omega$ ) \\
\hline Output voltage precision & $10 \mathrm{mV}$ \\
\hline Output voltage sign & $\begin{array}{l}50 \% \text { outputs supply positive voltages and the other } \\
50 \% \text { negative }\end{array}$ \\
\hline Operation mode & Mode 1: Self-calculation; Mode 2: PC-calculation \\
\hline
\end{tabular}

\subsubsection{Configuration of the designed heater controller}

According to the design requirements described in the last section, a dedicated heater controller was made, the configuration of which is shown in Figure 6.1. The essential components used in this configuration are listed in Table 6.2.

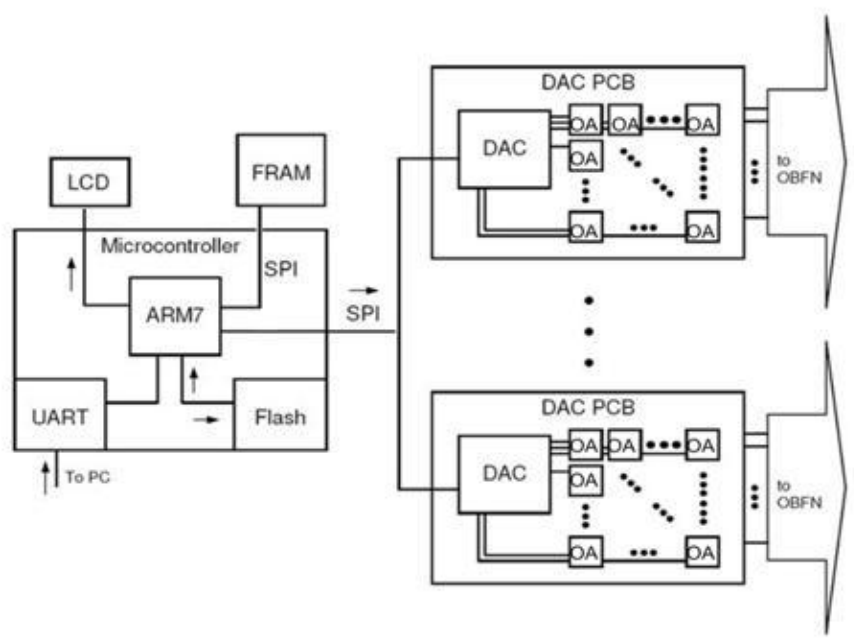

Figure 6.1: System configuration of the heater controller 
Table 6.2: Essential components of the heater controller

\begin{tabular}{|l|l|}
\hline Components & Descriptions \\
\hline Microcontroller & $\begin{array}{l}\text { NXP LPC2138 with 60 MHz ARM7 core, and } \\
512 \mathrm{kB} \text { on-chip Flash memory. }\end{array}$ \\
\hline Digital-analog converter (DAC) & $\begin{array}{l}32 \text {-channel, 14-bit DAC with output range } \\
{[0, \ldots, 5 \mathrm{~V}]}\end{array}$ \\
\hline Operational amplifier (OA) & $\begin{array}{l}\text { Boost factor 6; 50\% OAs for positive voltage } \\
\text { and the other 50\% negative }\end{array}$ \\
\hline Serial peripheral interface (SPI) & $\begin{array}{l}\text { Allowing data communication between one } \\
\text { microcontroller, multiple DACs, and storage } \\
\text { memory. }\end{array}$ \\
\hline $\begin{array}{l}\text { Ferroelectrical random access } \\
\text { memory (FRAM) }\end{array}$ & Storing and recalling heater tuning settings \\
\hline
\end{tabular}

\subsection{Heater voltage calculation}

To obtain the correct heater voltages for the desired beam angle of the PAA, the calculation steps listed in Table 6.3 must be conducted. The first step is to calculate the optical parameters ( $\kappa^{\prime} s$ and $\phi^{\prime} s$ ) of the beam former chip. In this step, the optical parameters are found by optimizing the generated delay responses of the ORR-based OBFN with respect to the desired delay responses derived from the beam angle $\theta$ [1], [2]. The desired and generated delay responses can be calculated using the PAA and ORR principles explained in Chapter 1 and 2. The formulas for the optimization process are given in Appendix A. In the second step, the $\kappa^{\prime} s$ and $\phi^{\prime} s$ are translated into the phase shifts of the tuning elements/optical phase shifters (OPSs) explained in Chapter 3. Based on the required phase shifts the corresponding heater voltages can be calculated using the heater principle explained in Chapter 5. However, these calculated heater voltages cannot directly be used. This is because thermal or electrical crosstalk may exist between the heaters due to the possible heat flow in the chip or the imperfection of the heater circuit. Therefore, in Step 3 a dedicated algorithm is used to adjust the heater voltages such that the crosstalk effect can be compensated. This algorithm is given in Appendix B. Furthermore, it is explained in Chapter 4 that the optical phase synchronization is required between the OBFN channels so that the signals of different channels can be combined coherently to achieve the maximum signal output power. Therefore, a dedicated OPS is used in each OBFN channel as the phase synchronizer. However, the optical phases are very sensitive to mechanical disturbances and ambient temperature changes, and therefore may fluctuate undesirably. To deal with this phase fluctuation, a feedback mechanism is used in Step 4 of the calculation process to adapt the corresponding OPSs continuously such that the optical phase fluctuation is corrected and the OBFN 
output power is optimized. The Genetic algorithm and Hill Climbing algorithm are used in this optimization process [3]. A block diagram showing these calculation steps is given in Figure 6.2.

Table 6.3: Heater voltage calculation steps

\begin{tabular}{|l|l|}
\hline Step 1 & $\begin{array}{l}\text { Calculate the optical parameters }\left(\kappa^{\prime} s \text { and } \phi^{\prime} s\right) \text { of the beam former chip } \\
\text { according to the desired beam angle of the PAA. }\end{array}$ \\
\hline Step 2 & $\begin{array}{l}\text { Translate the optical parameters into heater voltages according to the } \\
\text { principle of the optical tuning element. }\end{array}$ \\
\hline Step 3 & $\begin{array}{l}\text { Adjust the heater voltages to compensate the crosstalk between the } \\
\text { heaters. }\end{array}$ \\
\hline Step 4 & $\begin{array}{l}\text { Continuously adapt the heater voltages with a feedback mechanism to } \\
\text { overcome the fluctuation of the optical parameters. }\end{array}$ \\
\hline
\end{tabular}

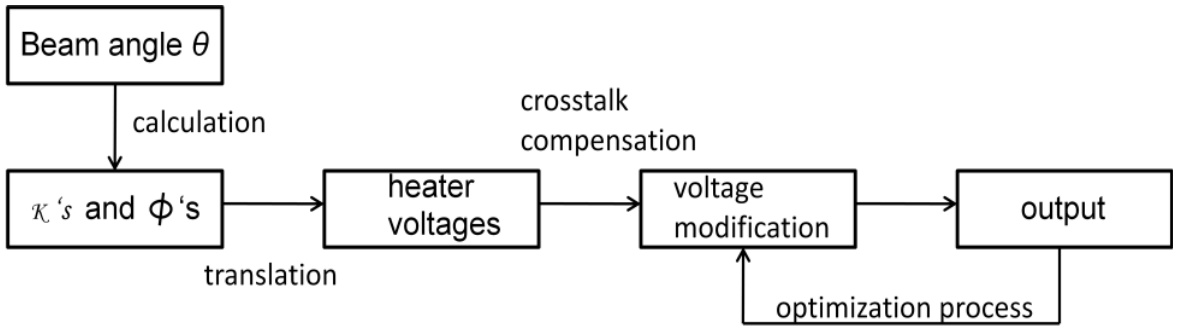

Figure 6.2: Block diagram of heater voltage calculation steps

\subsection{Chip temperature stabilization}

When the voltages are supplied to the heaters, the heat generated by the heaters may cause chip temperature change, which introduces optical phase fluctuation and consequently deviates the optical parameters of the chip. Therefore, a dedicated setup is needed under the chip to stabilize the chip temperature. A schematic of this setup is shown in Figure 6.4. A heat conducting base is placed under the chip with direct contact to the chip bottom side. Peltier elements and a negative temperature coefficient (NTC) resistor are placed under and inside the heat conducting base, respectively, and are connected to a temperature controller. This way, a close loop is formed as shown in Figure 6.4. Furthermore, the Peltier elements are placed on top of a cooling platform which works as the heat sink of the entire setup. The temperature controller receives the temperature feedback from the NTC resistor, and then adjusts the Peltier elements such that the chip temperature keeps stable. When using the heat conducting base with relatively large heat capacity, the chip temperature change is much less sensitive to the heat dissipated by the heaters, and so is the optical phase fluctuation. The relation between the optical phase fluctuation $\Delta \varphi$ and chip temperature change $\Delta T$ is given by: 


$$
\Delta \varphi=\frac{d n_{e}}{d T} \cdot \frac{d \varphi}{d n_{e}} \cdot \Delta T=\frac{\alpha \cdot 2 \pi \cdot L_{R}}{\lambda} \Delta T
$$

where $\alpha=d n_{e} / d T$ is the sensitivity of the effective index to the chip temperature; $d \varphi / d n_{e}=2 \pi \cdot L_{R} / \lambda$ is the sensitivity of the optical phase shift to the effective index; $L_{R}$ is the roundtrip length of the ORR and $\lambda$ is the wavelength. For the fixed value of total power dissipation, the larger the volume of the heat conducting base is, the smaller the chip temperature fluctuation is, and there the smaller the optical phase fluctuation is. Furthermore, the operation power limit of the Peltier elements must be larger than the maximum total power dissipation of the heaters, in order to guarantee the temperature stabilization. Based on the heater design mentioned in Chapter 5, the maximum power dissipation per heater is $0.5 \mathrm{~W}$. Therefore, the operation power limit of the Peltier elements can be chosen to be $20 \mathrm{~W}$ for the FLY chip (38 heaters), and $40 \mathrm{~W}$ for the SKY chip (79 heaters).

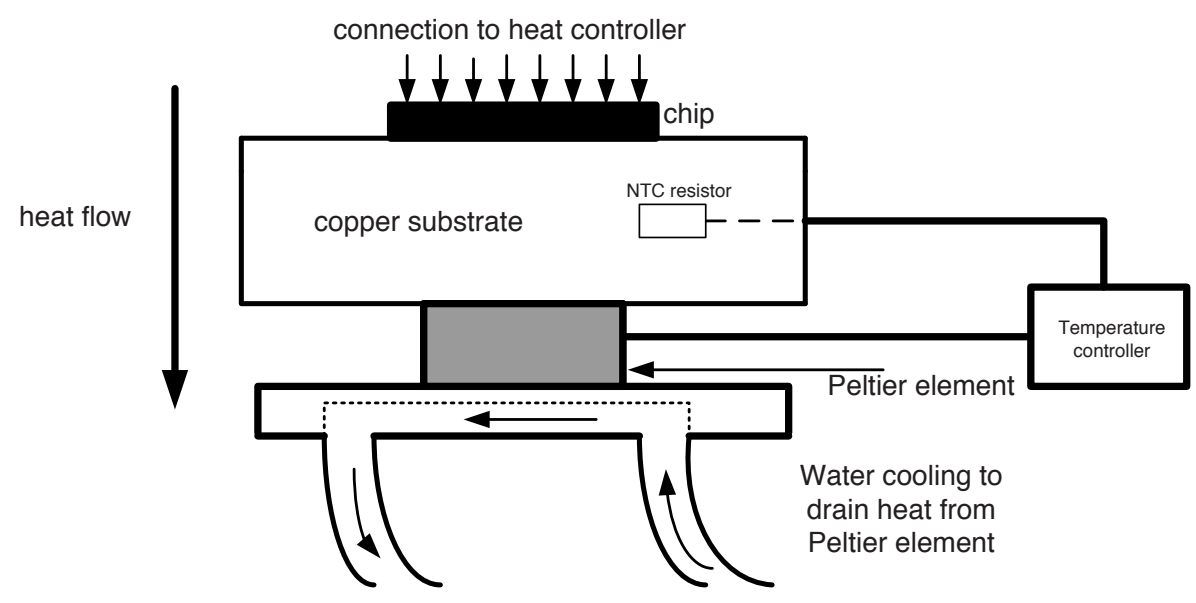

Figure 6.4: Schematic of the chip temperature stabilizing system

\subsection{Conclusions}

The beam former chip using thermal tuning mechanism requires a heater controller to perform the tuning process. The heat generated by the heaters may change the chip temperature and consequently cause optical parameter fluctuations. To solve this problem, a dedicated chip temperature stabilization setup is used, which consists of a heat conducting base, Peltier elements, a temperature controller, an NTC resistor and a heat sink. Then, when the operation power limit of the Peltier elements are larger than the maximum power dissipation of the heaters on the chip, the temperature of the chip can be kept stable. 


\section{References}

[1] J. W. van 't Klooster, C. Roeloffzen, A. Meijerink, L. Zhuang, D. Marpaung, W. van Etten, R. G. Heideman, A. Leinse, H. Schippers, J. Verpoorte, and M.Wintels, "Design of a ring resonator-based optical beam forming network for phased array receive antennas," Proceedings of the 30th ESA Antenna Workshop on Antennas for Earth Observation, Science, Telecommunication and Navigation Space Missions, 2008.

[2] R. J. Blokpoel, A. Meijerink, L. Zhuang, C. G. H. Roeloffzen, W. van Etten, "Staggered delay tuning algorithms for ring resonators in optical beam forming networks", Proceedings of the 12th Annual Symposium of the IEEE/LEOS Benelux Chapter, Brussels, Belgium, 17-18 December 2007, pp. 243-246.

[3] M. Burla, A. Garcia-Garcia, L. Zhuang, A. Meijerink, C. G. H. Roeloffzen, D. A. I. Marpaung, M. R. H. Khan, W. C. van Etten, "Optical phase synchronization in coherent optical beamformers for phased array receive antennas," IEEE LEOS Annual Meeting Conference Proceedings, 4-8 Oct 2009, Belek-Antalya, Turkey, pp. 693-694. 


\section{Chapter 7}

\section{Chip characterization}

\subsection{Introduction}

Two photonic beam former demonstrators using the realized FLY and SKY beam former chips as the core of the system were constructed at National Aerospace Laboratory (NLR) and Netherlands Institute for Radio Astronomy (Astron), respectively. The demonstrators are based on the practical architecture described in Chapter 4 . The purpose of these demonstrators is to verify the concepts described in the previous chapters. Therefore, the corresponding measurements on the demonstrators were performed. This chapter starts with the measurements on the elementary building blocks of the beam former chip. In this part the setup and steps for the chip measurement are first described, and then the measurement results on different components of the FLY and SKY chips, from the elementary building blocks to the system modules, are shown. Next, RF-to-RF measurements on the FLY beam former chip are presented. In this section, the desired functionalities of the photonic beam former system, such as optical sideband suppression, RF-to-RF delay generation, signal coherent combining between different beam former channels, and optical carrier reinsertion, are demonstrated. Finally, an experiment of antenna beam steering using the FLY photonic beam former demonstrator is presented at the end of this chapter.

\subsection{Characterization of beam former chip}

In this section the measurement setup and the characterization of the beam former chip are described. The chip characterization includes the measurement of the optical loss and the characterization on the components of the chip, such as the measurements of the extinction ratio of the Mach-Zehnder Interferometer (MZI) coupler, the group delay spectrum of the optical beam forming network (OBFN), and the power transfer function of the optical sideband filter (OSBF).

\subsubsection{Measurement setup}

For the characterization of the beam former chip, the frequency response measurement (power and group delay response) is an essential task. To perform this, a network analyzer (NA) is needed, which provides a single RF frequency signal to 
the system under test, and then obtain the response by comparing the corresponding output of the system to the input in both magnitude and phase. In our case the system under test not only includes the beam former chip but also an optical modulator and an optical detector for the $\mathrm{E} / \mathrm{O}$ and $\mathrm{O} / \mathrm{E}$ conversion as explained in Chapter 4. In order to obtain the frequency response of the beam former chip, one has first to calibrate the system to the state when the optical modulator and optical detector are directly connected to each other without the chip between them. Then, when the chip is added in the setup, its frequency response can be measured directly.

To obtain the frequency response of a certain part of the chip over the frequency range of interest, one way is to sweep the frequency of the NA signal over that frequency range. However, in this case the measurement frequency range is limited by the overlapping frequency range of the network analyzer, Mach-Zehnder modulator (MZM), and optical detector. In our setup, this overlapping frequency range is limited by the optical detector which works up to $12 \mathrm{GHz}$. More importantly, for this frequency sweeping approach, optical single sideband (SSB) modulation is required. Otherwise, for optical double sideband (DSB) modulation two sides of the modulated optical signal will experience the chip response at two different frequency positions, respectively, then after optical detection the resulted signal has the contributions from both frequencies, and therefore does not reflect the correct response of the chip. In comparison, optical SSB modulation results in only one sideband, which will experience the chip response at one frequency. However, as explained in Chapter 4, the implementation of optical SSB signal will increase the complexity of the measurement setup.

To achieve a measurement frequency range larger than the maximum frequency range of the measurement system (for example a measurement frequency range as large as the $14 \mathrm{GHz}$ FSR of the beam former chip) and in the meantime to achieve a lower complexity of the measurement setup, the wavelength-sweeping approach can be used instead of the single frequency-sweeping approach. In this case, the signal from the NA is fixed at a certain frequency, and different frequency positions in the required frequency range for the measurement are obtained by changing the wavelength of the optical carrier. When the frequency of the NA signal is much lower (for example 100 times lower) than the frequency range of interest in order to achieve good measurement resolution, optical DSB modulation can be applied to simplify the measurement setup because it requires only an MZM as explained in Chapter 4. Owing to the use of low frequency NA signal (for example, a $50 \mathrm{MHz}$ NA signal can be used for $6.7 \mathrm{GHz}$ measurement bandwidth which is the FSR of the OSBF), the required operation speed of the optical modulator and optical detector is significantly reduced, since there is no longer the need to match the bandwidth of 
those devices to the required measurement bandwidth. Figure 7.1 shows the setup for chip measurement, which is based on the wavelength-sweeping approach.

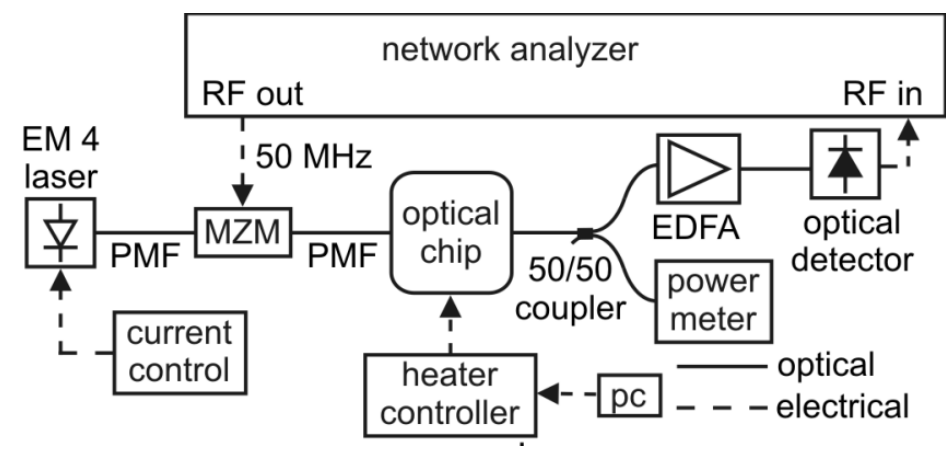

Figure 7.1: Measurement setup for beam former chip characterization.

Based on this setup, the RF amplitude and phase responses of the signal channels in the chip can directly be measured, and the group delay response can be obtained by means of the phase-shift method [3]. In the phase-shift method, an RF signal with a certain frequency $f_{\mathrm{RF}}$ is applied to the input of the system under test; then the network analyzer measures the phase shift $\Delta \phi_{\mathrm{RF}}$ of the RF signal between the output and the input of the system. In order to avoid RF phase ambiguity, the frequency $f_{\mathrm{RF}}$ is chosen such that $\Delta \phi_{\mathrm{RF}}<2 \pi$ results. Based on the phase shift $\Delta \phi_{\mathrm{RF}}$ and the frequency $f_{\mathrm{RF}}$, the corresponding group delay $\tau_{g}$ can be obtained by means of calculation. The corresponding formula for this calculation is given by

$$
\tau_{g}=\frac{\Delta \phi_{\mathrm{RF}}}{2 \pi f_{\mathrm{RF}}}
$$

Based on Eq. (7.1), the sensitivity of the group delay to the phase shift can be derived, which is given by

$$
S_{\tau_{g}}=\frac{d \tau_{g}}{d \Delta \phi_{\mathrm{RF}}}=\frac{1}{2 \pi f_{\mathrm{RF}}}
$$

It can be seen that the sensitivity increases when $f_{\mathrm{RF}}$ decreases. This implies that lower $f_{\mathrm{RF}}$ will lead to larger noise in the measured delay value for the same level of the phase noise in the measurement system. Therefore, when choosing $f_{\mathrm{RF}}$ for the measurement system, Eq. (7.2) should also be taken into account in order to guarantee the measurement accuracy.

In this measurement setup, the optical carrier is generated by an EM4 high-power 14-pin distributed feedback laser: EM253-080-057 with $80 \mathrm{~mW}$ maximum output power. The operation wavelength of the laser is in the region around $1550 \mathrm{~nm}$, and 
the linewidth of the laser $\Delta v$ is $1 \mathrm{MHz}$. According to [1], [2], the coherence time of the laser $\tau_{c}$ can be calculated by

$$
\tau_{c}=\frac{1}{\pi \Delta v}
$$

In our case $\tau_{c}=0.32 \times 10^{-6} \mathrm{~s}$. Multiplying $\tau_{c}$ by the speed of light $c_{0}$ produces the coherence length $L_{c}=\tau_{c} \cdot c_{0} \approx 100 \mathrm{~m}$, which is by far longer than the maximum optical path length difference of the beam former system, and hence satisfies the requirement on the laser source as explained in Chapter 4. To achieve wavelength tunability of the laser, the method of changing the injection current is used. An Avanex PowerLog FA-20 MZM is used to modulate the optical carrier, which has an operation bandwidth of $20 \mathrm{GHz}$. The modulating signal is a $50 \mathrm{MHz}$ single frequency signal generated by an Agilent N5230A PNA-L network analyzer. This frequency is chosen to achieve both the measurement accuracy and easiness as explained in the beginning of this section. Because of the polarization dependency of the waveguide of the chip (explained in Chapter 5), PMF connections are used between the laser output, modulator, and chip input, so that the chip is characterized with the required transverse-electric-polarized light whose polarization lies in the Xaxis direction of the waveguide cross-section. As explained in Chapter 5, different parts of the chip can be measured separately by accessing the corresponding input and output ports of the chip. The heater controller is connected to the chip for the tuning operation. At the output of the beam former chip, a $2 \times 23-\mathrm{dB}$ optical fiber coupler is used to split the output signal into two paths. One fiber output is connected to a Hewlett Packard E8153A optical power meter, and the other is used for RF detection. The RF signal is detected using a $12 \mathrm{GHz}$-bandwidth Emcore 2860 E optical detector with a build-in transimpedance amplifier, and led to the network analyzer for measurement. If the optical signal is too low for detection due to the losses in the chip and the other parts of the optical path (details of optical losses in the beam former chip will be further explained in Section 7.2.7), an erbium-doped fiber amplifier (EDFA) can be used to boost the optical power.

\subsubsection{Measurement steps}

The performance of the beam former chip depends on the correct functioning of its building blocks. When a building block is malfunctioning, the total device will not work either. Therefore, several test chips with the separate building blocks for different parameters were fabricated in the same waveguide technology before the design and fabrication of the beam former chip, so that those building blocks could be individually characterized and the measurement results have been used to optimize the beam former chip. The measurement steps to characterize a chip are listed in Table 7.1. 
Table 7.1: Chip characterization steps

\begin{tabular}{|c|c|c|}
\hline Step & Task & Descriptions \\
\hline 1 & $\begin{array}{l}\text { Check the functionality } \\
\text { of the components in the } \\
\text { measurement setup. }\end{array}$ & $\begin{array}{l}\text { Test if the components provide the required } \\
\text { functionality as their datasheets describe and if } \\
\text { they have sufficient stability to guarantee } \\
\text { accurate measurements. }\end{array}$ \\
\hline 2 & $\begin{array}{l}\text { Check the quality of the } \\
\text { in- and out-couple fibers } \\
\text { (in case of unpigtailed } \\
\text { test chips). }\end{array}$ & $\begin{array}{l}\text { First, measure the output power or spectrum of a } \\
\text { single-fiber link. Then, measure the output } \\
\text { power or spectrum of the link formed by the in- } \\
\text { couple fiber to out-couple fiber, where the } \\
\text { centers of two cleaved fibers are aligned and } \\
\text { brought into contact. The fibers must be re- } \\
\text { cleaved when there is a large difference ( }>0.2 \\
\text { dB) with respect to the first measurement. }\end{array}$ \\
\hline 3 & $\begin{array}{l}\text { Measurement of the chip } \\
\text { insertion loss. }\end{array}$ & $\begin{array}{l}\text { The chip insertion loss consists of the } \\
\text { propagation loss of the waveguide and the } \\
\text { coupling losses between fibers and chip. }\end{array}$ \\
\hline 4 & $\begin{array}{l}\text { Measurement of the } \\
\text { extinction ratio of the } \\
\text { bar-port and cross-port } \\
\text { of tunable MZI couplers }\end{array}$ & $\begin{array}{l}\text { First check the spectrum of the coupler output. } \\
\text { Then, the maximum and minimum bar-port } \\
\text { output power of an MZI coupler can be reached } \\
\text { by changing the phase difference between the } \\
\text { two MZI arms. The cross-port is complementary } \\
\text { to the bar-port. }\end{array}$ \\
\hline 5 & $\begin{array}{l}\text { Measurements of the } \\
\text { group delay of the } \\
\text { ORRs. }\end{array}$ & $\begin{array}{l}\text { The group delay responses of the OBFN } \\
\text { channels can be obtained by means of the phase- } \\
\text { shift method. }\end{array}$ \\
\hline 6 & $\begin{array}{l}\text { Measurement of the } \\
\text { power transfer of the } \\
\text { OSBF. }\end{array}$ & $\begin{array}{l}\text { The power transfer of the OSBF can be obtained } \\
\text { by performing wavelength step measurement } \\
\text { with optical power meter [1]. }\end{array}$ \\
\hline
\end{tabular}

\subsubsection{Measurement of the insertion loss of the chip}

The insertion loss is an important criterion for the performance of the chip, the value of it determines the usability of the realized chip. To measure the insertion loss accurately, all the MZI couplers in the measured waveguide channel should be tuned such that the loss is minimized. This means the bar-port connections are required for the MZI couplers. This is because when a tunable MZI coupler has two DCs with equal coupling coefficient, the MZI can be tuned to have $100 \%$ power transmission via bar-port connection as explained in Section 2.3.2. For instance, the measurement on the FLY beam former chip (the design of which is described in Chapter 4) shows the minimum insertion loss of approximately $12 \mathrm{~dB}$, which is based on a channel including only one MZI coupler (in bar state) in its path. In this case the light is coupled into the top channel of the OBFN which has no ORR as 
shown in Figure 5.1, and then after the first MZI coupler, the light is coupled out of the chip via the MZI bar-port output. The chip insertion loss has the contributions of two times the coupling loss between fiber and chip (fiber-chip and chip-fiber coupling loss), and propagation loss in the waveguide. In principle the values of these two kinds of losses can be obtained by measuring the output power of different lengths of the waveguide (cutback method). This must be a straight line in a logarithmic graph where the slope gives the waveguide propagation loss in $\mathrm{dB} / \mathrm{cm}$. When this straight line is normalized to the output power of the fiber-to-fiber measurement, the extrapolated value at zero waveguide length gives the total losses of fiber-chip and chip-fiber couplings. For the beam former chip, the values of fiberchip coupling loss and waveguide channel loss can also be calculated from the lossdelay relation of a ring resonator. This calculation will be further described in Section 7.2.7.

\subsubsection{Characterization of the MZI coupler}

Since the tuning range of the MZI couplers determines the tuning range of power coupling coefficient $\kappa$ of the ORRs, measurements were carried out on a test waveguide where a tunable MZI coupler was connected via its bar ports (as explained in Chapter 2, the tuning range of $\kappa$ of the ORRs can be calculated from the bar-port power extinction ratio of the MZI). Although not shown here, the spectrum of the MZI coupler output appears to be approximately constant over the $14 \mathrm{GHz}$ measurement bandwidth. This is because the DCs that constitute the MZI couplers can be regarded as frequency-independent devices when the frequency range is in the order of tens of gigahertz. The measured bar-port power extinction ratio of the MZI coupler $\eta$ in $\mathrm{dB}$ is approximately $13 \mathrm{~dB}$, corresponding to a minimum power transmission of 0.06 . This means that the tuning range of the power coupling coefficient $\kappa$ of the ORRs (tuning range of the cross-port power transmission of the MZI couplers) would be from 0 to $0.94(=1-0.06)$. Based on Eq. (2.16), one can find out the power coupling coefficient of the DC $\kappa_{\mathrm{DC}}$ from the measured value of $\eta$ (in $\mathrm{dB}$ ). The corresponding equation is given below

$$
\kappa_{\mathrm{DC}}(\eta)=\frac{1}{2}\left(1 \pm 10^{\frac{\eta}{20}}\right)
$$

In our case $\kappa_{\mathrm{DC}}=0.38$ or 0.62 . This is because the DC with coupling coefficient of $\kappa_{\mathrm{DC}}$ or $1-\kappa_{\mathrm{DC}}$ results in the same value of $\eta$ as can be seen in Section 2.3.2. Moreover, $\eta$ also indicates the capability of the optical combiners in the chip to compensate the power difference between its two input ports. In this case the optical combiners are capable to compensate power difference up to approximately $13 \mathrm{~dB}$. 


\subsubsection{Characterization of the ORR-based OBFN}

As explained in Chapter 3, the most important function of the OBFN is to synchronize the signals from different AEs by providing proper delays. For the verification of the OBFN delay generation, several test ORRs and OBFNs were realized before the fabrication of the final beam former chip. Those test chips were fabricated using the same waveguide technology and with the same ORR dimensions as the final beam former chip, in order to make sure that the measured ORR performances are repeatable in the final beam former chip.

First, several group delay measurements were performed on one, two, and three cascaded ORRs. The results are shown in Figure 7.2, 7.3, and 7.4, respectively, which demonstrate the tunability of the ORR-based delay elements and the inherent trade-off between the peak delay, bandwidth, and delay ripples. In the case of three cascaded ORRs, flattened group delay responses were achieved with a maximum delay value of approximately $1.3 \mathrm{~ns}$ (which corresponds to $39 \mathrm{~cm}$ physical distance in air) for a bandwidth of $0.6 \mathrm{GHz}$ and a maximum delay ripple of approximately 50 ps (1.5 cm physical distance in air). The group delay measurements shown in Figure 7.5 demonstrate the "squeezing" method for delay ripple reduction, which is explained in Chapter 2. It is shown that the delay ripples can be reduced at the cost of bandwidth. Figure 7.6 shows a group delay response of the three cascaded ORRs and the corresponding power transfer. It can be seen that the power transfer expressed in $\mathrm{dB}$ varies in the same manner as the group delay response, which verifies the loss-delay relation described in Chapter 2: the delay is generated by the structural enhancement of the delay path length in the ORR, and the loss due to the waveguide attenuation is proportional to this delay path length.
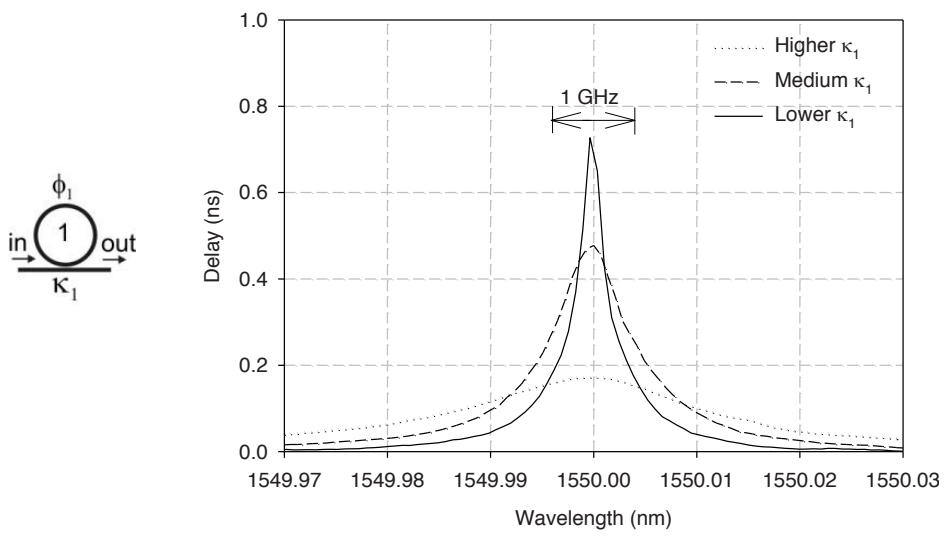

Figure 7.2: Measured group delay responses of one ORR for different values of $\kappa_{1}$ 


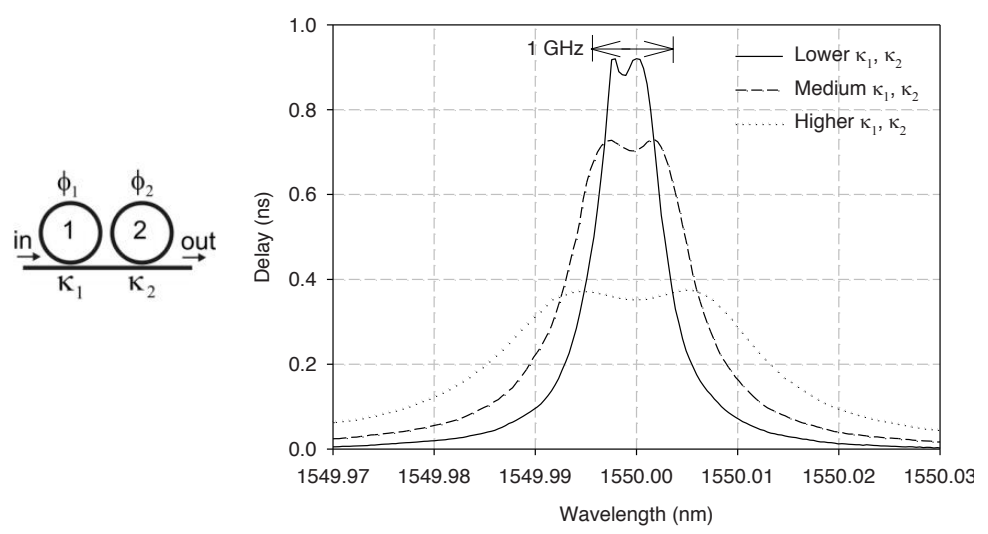

Figure 7.3: Measured group delay responses of two cascaded ORRs for different coupling strengths of the two couplers
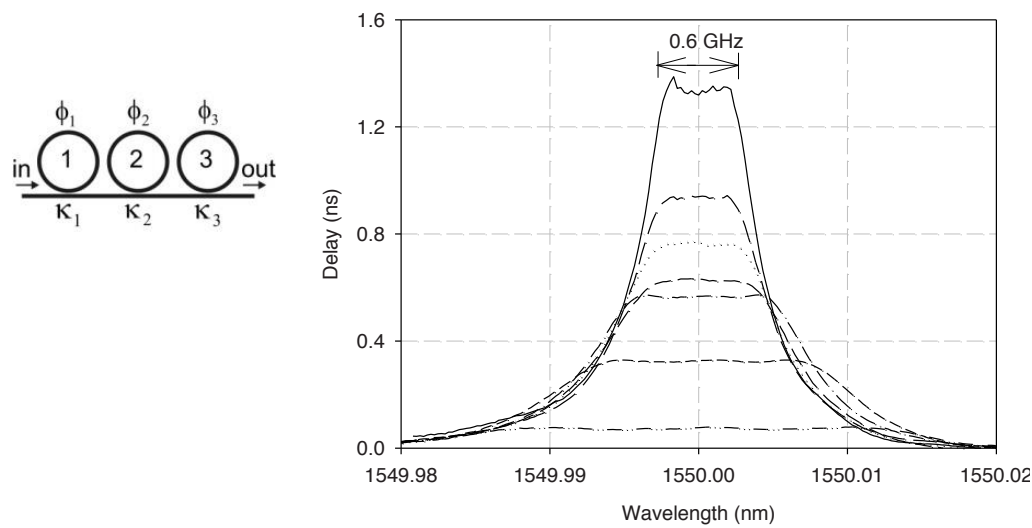

Figure 7.4: Different delay values measured on three cascaded ORRs 


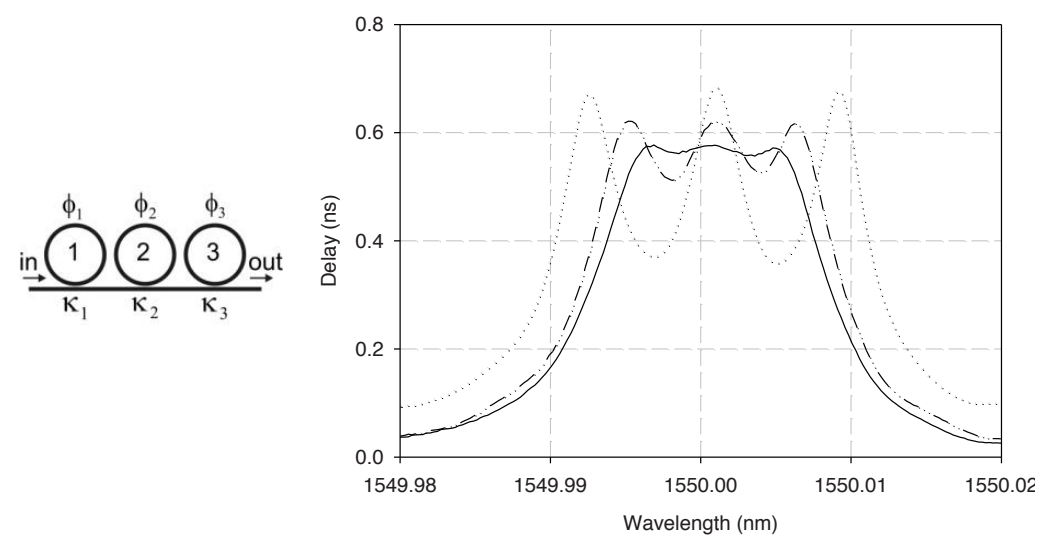

Figure 7.5: Demonstration of the delay ripple reduction of a cascade of three ORRs
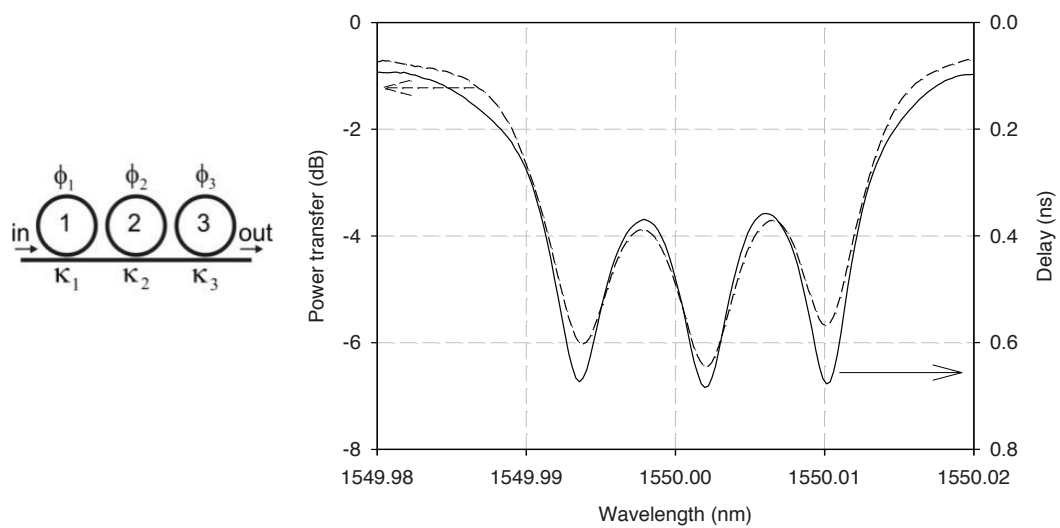

Figure 7.6: Demonstration of the loss-delay relation of a cascade of three ORRs 
After the measurements on separate ORR-based delay elements, several measurements were performed on an OBFN test chip. The chip contains an $1 \times 8$ asymmetrical binary-tree structured OBFN (which can also be used as $8 \times 1 \mathrm{OBFN}$ due to device reciprocity). The OBFN is designed with linearly increasing number of ORRs in each stage, which guarantees that the OBFN channels are able to provide linearly increasing delay values for linear PAA applications. The architecture of the OBFN and the corresponding waveguide layout of the chip are shown in Figure 7.7 (a) and (b), respectively.

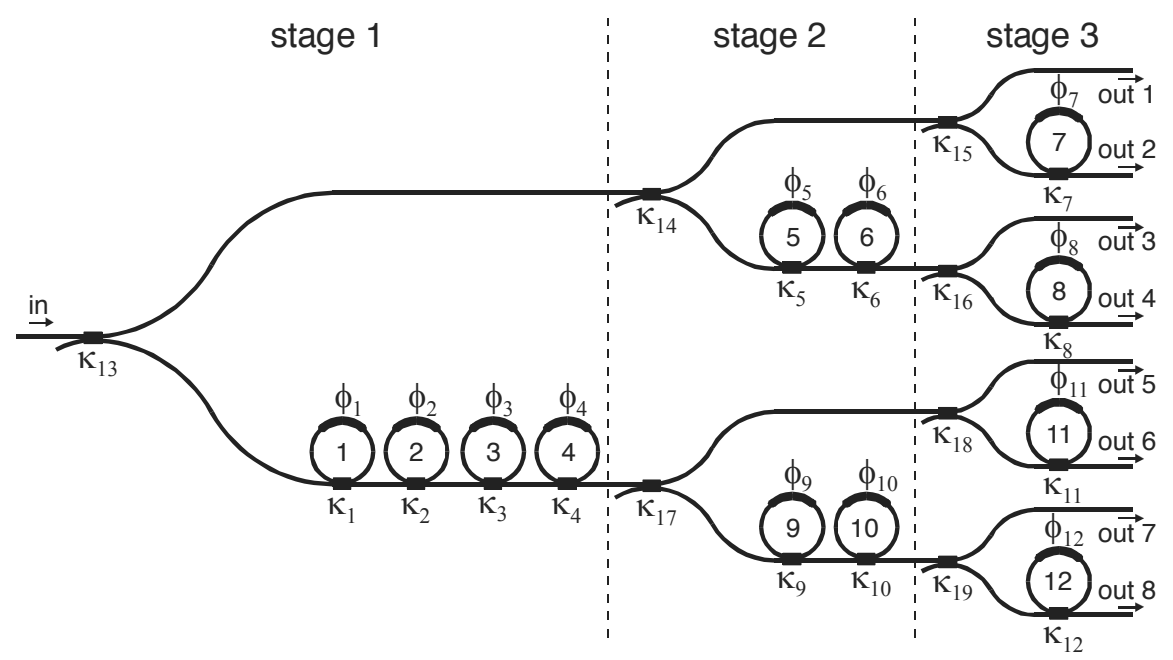

(a)

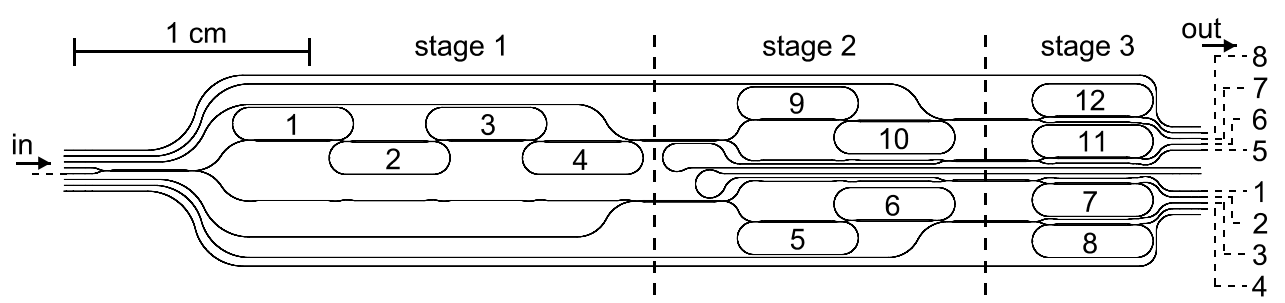

(b)

Figure 7.7: (a) Architecture of the $1 \times 8 \mathrm{OBFN}$; (b) the corresponding waveguide layout of the test chip.

Figure 7.8 demonstrates that group delay response at output 8 for the channel with three ORRs (ORR 9, 10, and 12) is the sum of the group delay responses of two individual delay sections: two cascaded ORRs (ORR 9 and 10) and ORR 12. The delay section with two cascaded ORRs was separately measured by using the lower input of coupler 17 and out 7; ORR 12 was accessed from the lower input of coupler 
19. Note that, due to the measurement approach, the measured group delay response of each individual delay section contains not only the contributions of ORRs but also the contribution of a certain length of straight waveguide lying between the input and output of the measured channel. Therefore, in Figure 7.8 the peak delay of three ORRs is slightly lower than the sum of the peak delays of the two individual delay sections. To verify the accuracy of the delay measurements on the OBFN channels, a delay setting for three outputs of the OBFN (out 2, 3, and 4) were created, and then the measured group delay responses at the three outputs were compared with the corresponding simulation results, which are shown in Figure 7.9. The comparison shows a good agreement between the measured and simulated group delay values.

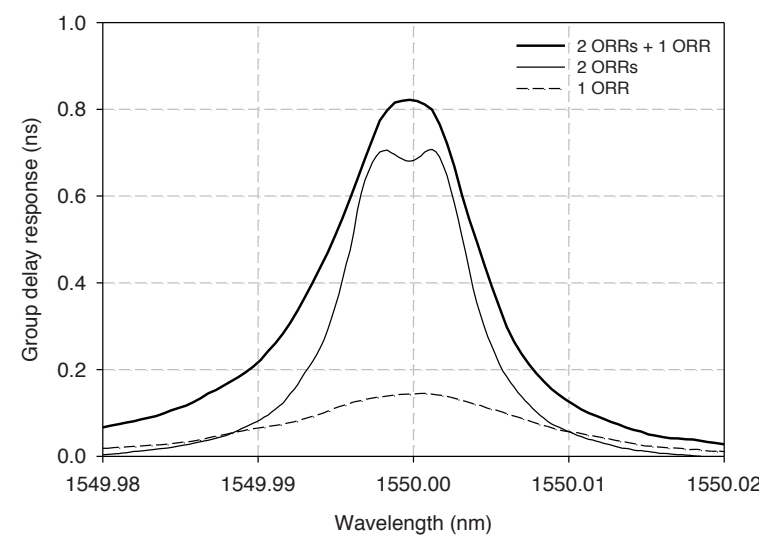

Figure 7.8: Measured OBFN channel group delay responses at output 4 and the two individual delay sections in the corresponding delay path

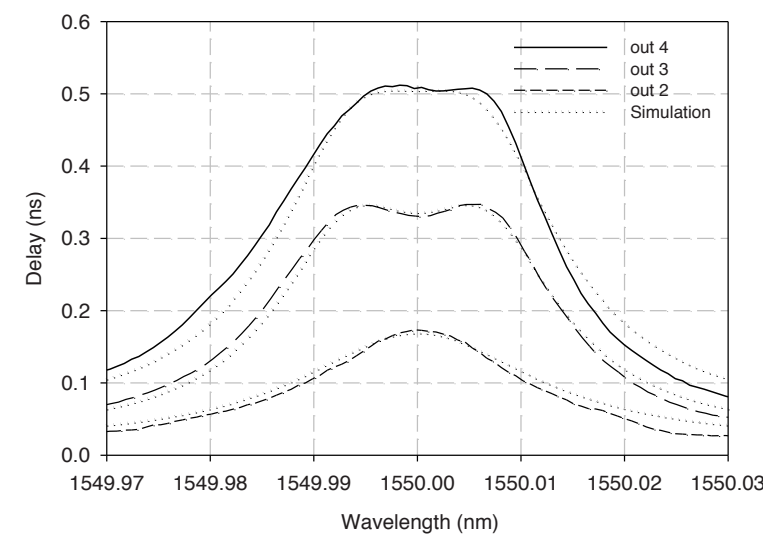

Figure 7.9: Comparison between measured and simulated group delay responses 
Furthermore, a delay setting for the complete OBFN was made by properly tuning the ORRs in each delay section in order to demonstrate the desired OBFN functionality. The achieved delay setting is shown in Figure 7.10, which provides linearly increasing delays for out 1 to out 8 at the same time. The achieved delay responses of 8 channels cover a common delay band. Out 1 is connected to the OBFN channel without ORR, and is considered here as the zero-delay reference.

This delay setting corresponds to the application of an 8-element linear PAA. The result also shows that a delay value of around $1.2 \mathrm{~ns}$ ( $36 \mathrm{~cm}$ physical distance in air) can be achieved with 7 cascaded ORRs for a bandwidth of $2.5 \mathrm{GHz}$ and the maximum delay ripple of approximately $0.1 \mathrm{~ns}(3 \mathrm{~cm}$ physical distance in air).

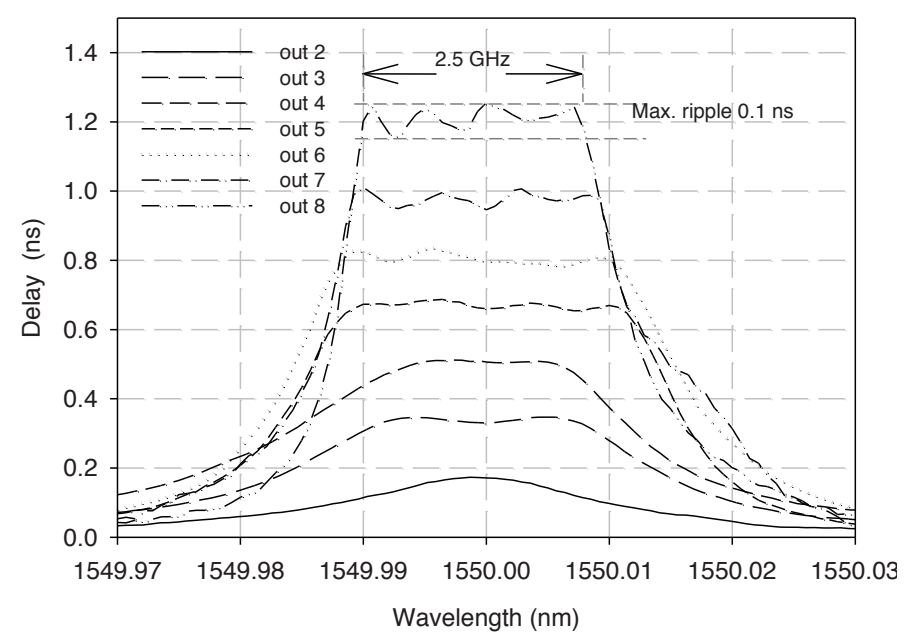

Figure 7.10: A delay setting of the $1 \times 8$ OBFN in the test chip

\subsubsection{Characterization of the OSBF}

With the same approach as for the OBFN, an OSBF test chip was fabricated before the final design of the beam former chip. For practical reasons, the test OSBF was designed with a smaller ORR and a corresponding shorter path length difference between the MZI arms than the required ones for FLY and SKY beam former chip (see Chapter 4). Thus, a larger FSR of $40 \mathrm{GHz}$ resulted. Figure 7.11 shows both the measured and simulated OSBF power transfer, and the measurement shows good agreement with the simulation. The measurement proves that this type of filter (MZI + ORR) is able to provide a flat passband and $25 \mathrm{~dB}$ stopband suppression for the bandwidth characteristics: $-3 \mathrm{~dB}$ passband accounts for approximately $50 \%$ of the FSR, transition band $7.5 \%$, and stopband $35 \%$ at $-25 \mathrm{~dB}$. 


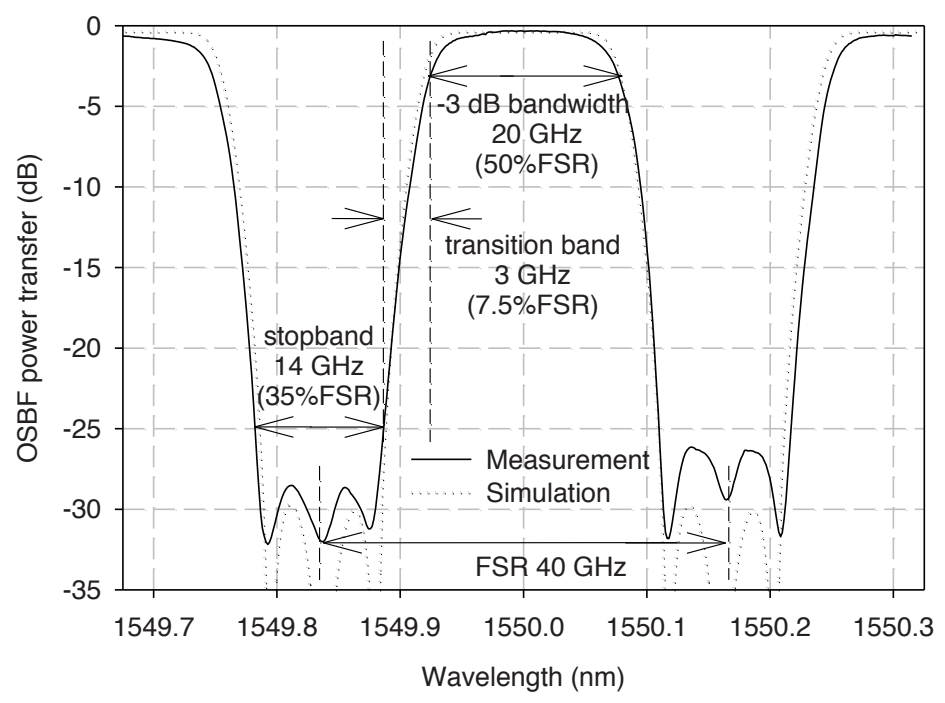

Figure 7.11: Measured and simulated OBFN power transfer (according to $0.5 \mathrm{~dB} / \mathrm{cm}$ waveguide loss)

\subsubsection{Characterization of the FLY beam former chip}

\section{- OBFN}

As described in Chapter 4, the FLY beam former chip is designed for an 8-element linear PAA. Therefore, the OBFN is required to provide linearly increasing delays on channel 1 to channel 8 (see Figure 4.14). In order to create proper OBFN delay settings for the beam forming purpose, every channel was first tuned to its minimum delay. Next, the achieved minimum delay was regarded as the zero delay reference of the corresponding channel. As explained in Chapter 3, the common zero-delay plane for the OBFN channels can be achieved by using additional fixed delay offsets in the RF or optical paths. The corresponding delay setting (zero delay for all channels) was regarded as the minimum delay setting. Based on the minimum delay setting, another two delay settings were created, which are shown in Figure 7.12 (a) and (b). These two delay settings provide linearly increasing delays over the optical bandwidth of $1 \mathrm{GHz}$, which correspond to two different beam angles for the antenna. The delay steps between two neighboring channels for the two settings are approximately $20 \mathrm{ps}$ and $40 \mathrm{ps}$, respectively, which correspond to physical distances of $0.6 \mathrm{~cm}$ and $1.2 \mathrm{~cm}$ between two neighboring AEs. 


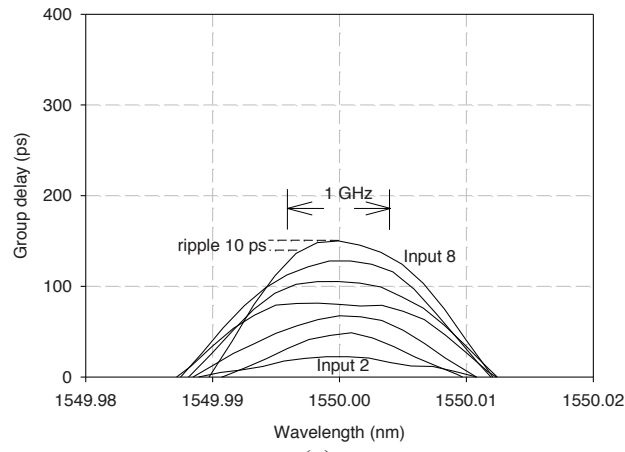

(a)

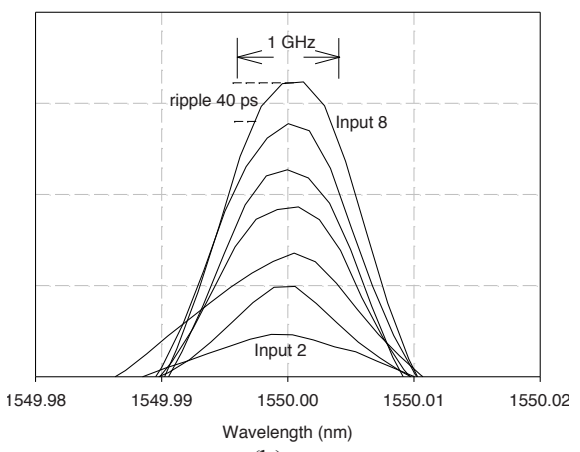

(b)

Figure 7.12: Two delay settings of the FLY OBFN with delay step size of (a) $20 \mathrm{ps}$ and (b) $40 \mathrm{ps}$ between the neighboring channels.

As described in Chapter 4, the FLY antenna has an AE spacing of approximately $1.2 \mathrm{~cm}$. if one of these settings is used for the 0 degree beam angle (broadside), the other setting would steer the beam by approximately 30 degree. Note that the achieved bandwidth in the second delay setting in Figure 7.12 is smaller than the designed $1.2 \mathrm{GHz}$. This is because the achieved tuning range for $\kappa$ of the ORRs is not the desired $[0,1]$ but a smaller range $[0,0.94]$ as mentioned in Section 7.2.2, which resulted in a $40 \mathrm{ps}$ increase in the minimum delay of the ORR-based delay elements. Hence, the ORRs needed to provide higher maximum delays than in theory to achieve a desired delay tuning range, which eventually resulted in the smaller delay bandwidths.

\section{- OSBF}

As described in Section 4.11, the OSBF of the FLY beam former is designed for a FSR of $6.7 \mathrm{GHz}$. The measured OSBF power transfer is presented in Figure 7.13. The passband, transition band, and stopband have widths of approximately $3 \mathrm{GHz}, 1$ $\mathrm{GHz}$, and $1.7 \mathrm{GHz}$, respectively, and the stopband suppression is $25 \mathrm{~dB}$, as desired. However, the measured width of the stopband is slightly smaller than the theoretical width $(30 \% \mathrm{FSR}=2 \mathrm{GHz})$. This is because the achieved filter parameters have slight deviations from the simulated values. As explained in Section 4.11, the FSR of the ORR should be half of that of the asymmetrical MZI, and correspondingly the roundtrip length of the ORR should be twice the length difference between the two MZI arms. However, in the fabricated chip the ORR and the asymmetrical MZI of the OSBF can have small optical path length deviations. This optical path length deviation can be established from the period of the additional modulation on the OSBF power transfer. The measured OSBF power transfer over $4 \mathrm{~nm}$ wavelength range is shown in Figure 7.14 (a), which appears to be periodically modulated. The 
period is about $125 \mathrm{GHz}$, which is roughly 20 times as large as the designed FSR of 6.7 GHz. Using the theory described in Section 4.11, one can conclude that there is an optical path length deviation of the ORR or of the MZI, which is about $5 \%(1 / 20)$ of the designed unit delay length of the OSBF (length difference between two MZI arms). Furthermore, the close-up version of the OSBF power transfer in Figure 7.14 (b) shows that the distance between two neighboring dips (resonances of the ORR) is less than the designed FSR of $6.7 \mathrm{GHz}$, which means that the roundtrip length of the ORR $L_{R}=c_{0} / n_{g} \cdot \Delta f_{\mathrm{FSR}}$ is longer than the designed value, according to Eq. (2.9). This is probably because the bends of the ORR cause a small deviation in $n_{g}$. As a consequence, the width of the stopband of the OSBF had to be slightly decreased for the desired $25 \mathrm{~dB}$ suppression. However, this is not a problem for the system functionality, as the required bandwidth is only $1.2 \mathrm{GHz}$.

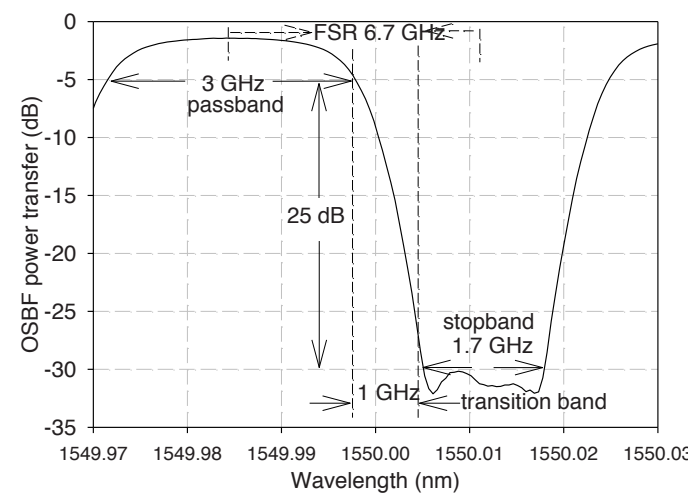

Figure 7.13: Optimized OSBF power response of FLY chip

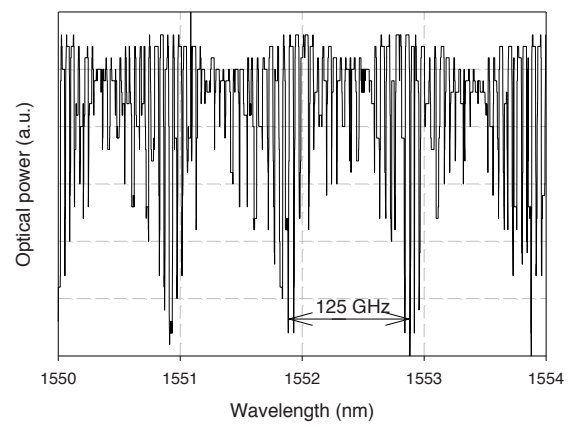

(a)

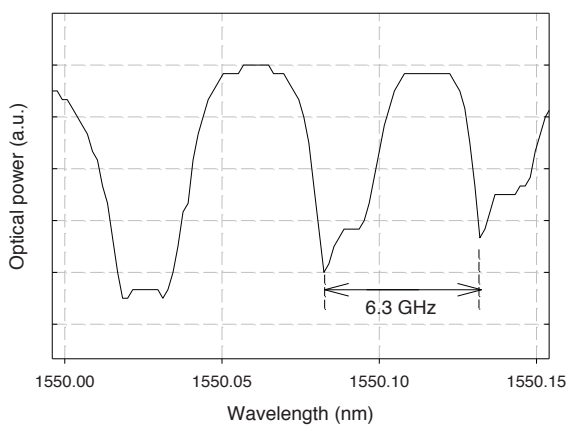

(b)

Figure 7.14: (a) OSBF power transfer; (b) close-up version of the OSBF power transfer. 


\section{- Optical losses in the chip}

As mentioned in Section 7.2.1, the waveguide loss of the beam former chip can be estimated by means of the cutback method. However, this method requires destructive measurement on the chip: the waveguide length is adjusted each time by cutting part of the chip off. Alternatively, a non-destructive way to estimate waveguide loss is to use the relation between delay and loss of ORR (which has been explained in Chapter 2). According to this relation, the waveguide loss $P_{\mathrm{L}_{-} \text {ch }}$ of the beam former chip can be estimated by

$$
P_{\mathrm{L}_{-} \mathrm{ch}}=-\frac{\Delta P_{\text {out } \_ \text {ch }} \cdot n_{\mathrm{eff}}}{\Delta \tau_{\mathrm{g} \_ \text {ch }} \cdot c_{0}}
$$

where $P_{\text {out_ch }}$ and $\tau_{\mathrm{g} \_c h}$ are the measured optical power in $\mathrm{dB}$ and group delay at the output of an OBFN channel, respectively. Figure 7.15 shows the measurement results of $P_{\text {out_ch }}$ and $\tau_{\mathrm{g} \_c h}$ of an OBFN channel with one ORR in its path. Based on this measurement, the waveguide loss is estimated to be approximately $0.6 \mathrm{~dB} / \mathrm{cm}$. Then, by subtracting the total waveguide loss $0.6 \mathrm{~dB} / \mathrm{cm} \times 7 \mathrm{~cm}=4.2 \mathrm{~dB}$ from the measured minimum insertion loss of $12 \mathrm{~dB}$, the total coupling loss is estimated to be roughly $8 \mathrm{~dB}$ ( $4 \mathrm{~dB}$ coupling loss per facet).

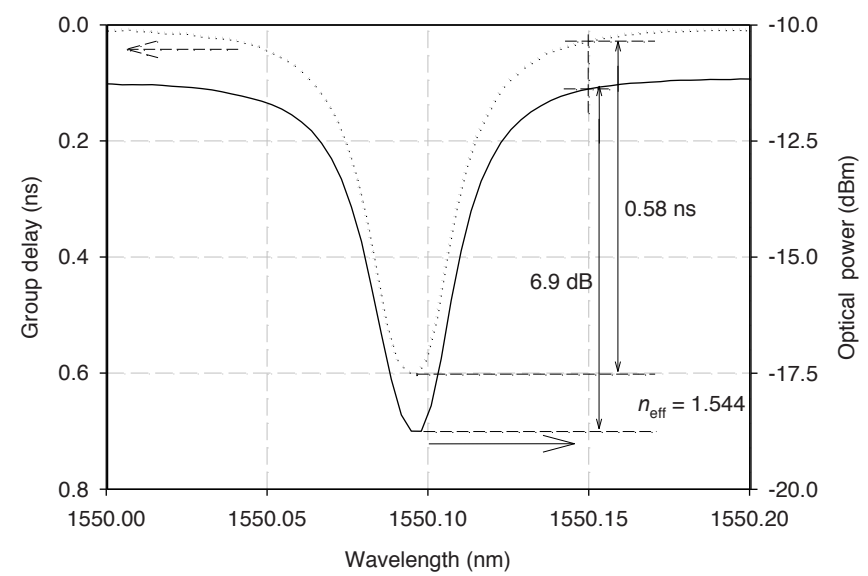

Figure 7.14: Measured optical power and group delay at the output of an OBFN channel

Furthermore, the relation between delay and loss also implies that the maximum insertion loss of the chip occurs when the delay elements in the OBFN channel are tuned to their maximum delay setting. For example, if input 8 of the OBFN is tuned to a total maximum delay of $560 \mathrm{ps}$ (which corresponds to roughly $11 \mathrm{~cm}$ distance in the chip), the total additional loss contributed by the ORRs is roughly $7 \mathrm{~dB}$. This was verified by measurements. Due to the propagation loss in the waveguide, the passband loss of the OSBF is roughly equal to $2 \mathrm{~dB}$ as shown in Figure 7.13. 
Therefore, the total optical loss (loss from both OBFN and OSBF) in different channels of the chip, excluding intrinsic splitting and combining losses, can vary from roughly 14 to $21 \mathrm{~dB}$.

To overcome this optical loss, an EDFA was used after the chip output, which boosts the optical power by $20 \mathrm{~dB}$. However, this would certainly not be desirable in a final photonic beam former product, because of the associated additional costs, and also because of the additional noise that would be introduced. However, recent improvement in the waveguide technology has resulted in chips for different purposes with waveguide loss well below $0.1 \mathrm{~dB} / \mathrm{cm}$ [4], [5], and by effectively tapering the waveguides, the fiber-chip coupling losses to well below $1 \mathrm{~dB}$ per facet [5]. Therefore, in the future the beam former chip will have better performance with respect to optical losses. An even better solution is to completely avoid fiber pigtailing and directly integrate light sources and detectors via an integration technology such as flip-chip technology.

Moreover, the optical combiners in this chip are good enough to compensate the loss differences between the combining branches, since for the maximum delay setting, the resulted maximum loss difference between combining branches in this chip is roughly $4 \mathrm{~dB}$ (which may occur at the last optical combiner before the OBFN output, shown in Figure 4.14), and the optical combiners are capable to compensate power differences up to approximately $13 \mathrm{~dB}$ as described in Section 7.2.4.

\subsubsection{Characterization of the SKY beam former chip}

\section{- OBFN}

Using the same principle as for the FLY chip, first a minimum delay setting was made for the SKY OBFN, and the minimum delay in each channel was regarded as the zero delay reference of that channel. When a common zero-delay plane is achieved for all the channels (the differences between those minimum delays are compensated in the RF signal paths). Next, based on the mapping between the AEs and OBFN inputs shown in Figure 4.19, another OBFN delay setting for demonstrating the beam steering ability of the antenna was created. The measured group delay responses of the OBFN channels are shown in Figure 7.15, where each sub-figure shows the group delay responses for a different column of AEs in the array (see Figure 4.19). For each column of AEs, the corresponding delays increase linearly from the minimum one (thick line) with the step size of approximately 110 ps $(3.3 \mathrm{~cm}$ physical distance in air) for the delay bandwidth of approximately $1 \mathrm{GHz}$. This corresponds to the beam steering in the angle plane which is parallel to the $\mathrm{AE}$ columns. Furthermore, the minimum delays (thick lines) for AE column 1 to 4 are also tuned to increase linearly with the step size of approximately $110 \mathrm{ps}$. This 
facilitates the beam steering in the angle plane which is perpendicular to the $\mathrm{AE}$ columns. This way, the 2-D beam steering is achieved.

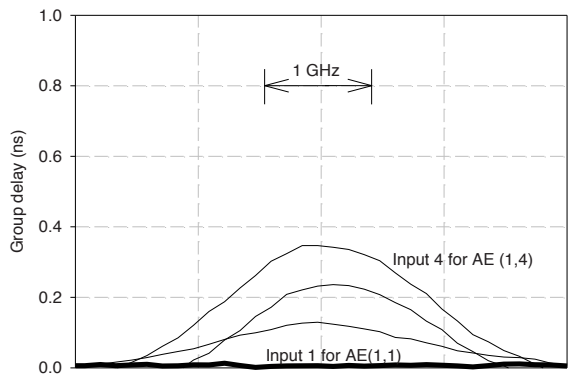

(a)

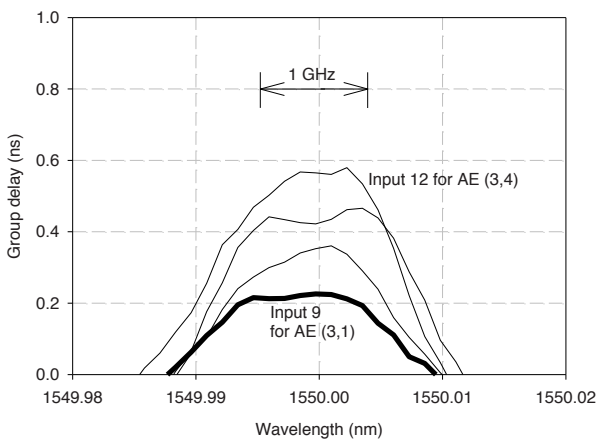

(c)

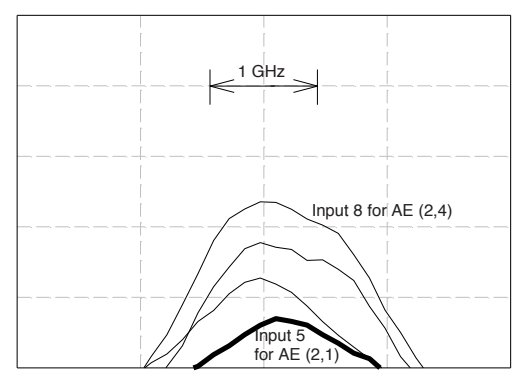

(b)

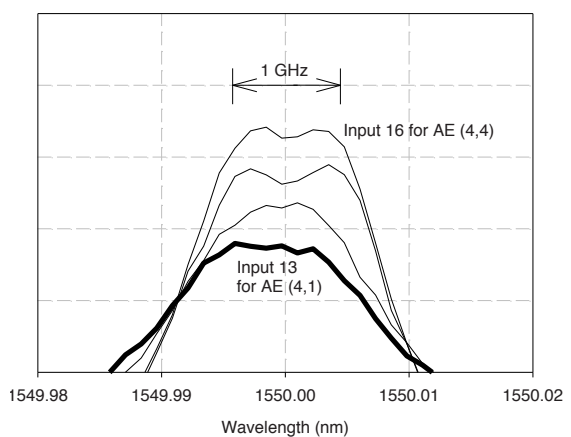

(d)

Figure 7.15: Group delay responses of the SKY OBFN channels for 2-D beam steering; (a) to (d) shows the group delay responses for AE column 1 to 4 (see Figure 4.19), respectively.

\section{- OSBF}

As described in Section 4.12, the OSBF on the SKY beam former chip is designed for a FSR of $4 \mathrm{GHz}$. The optimized filter power transfer is presented in Figure 7.16. Using the same principle as for the FLY OSBF, the response is normalized to the response corresponding to the input-output configuration that skips the OSBF in the path. The measured passband, transition band, and stopband have widths of approximately $1.7 \mathrm{GHz}$ at $-5 \mathrm{~dB}, 0.55 \mathrm{GHz}$, and $1.2 \mathrm{GHz}$ at $-27 \mathrm{~dB}$, respectively, and the stopband suppression is $25 \mathrm{~dB}$. Similar to the FLY OSBF, the measured bandwidth characteristics are slightly different from the theory, which is probably because of the deviation in $n_{g}$. However, this is not a problem for system functionality since the filter response still satisfies the $1.2 \mathrm{GHz}$ bandwidth requirement. 


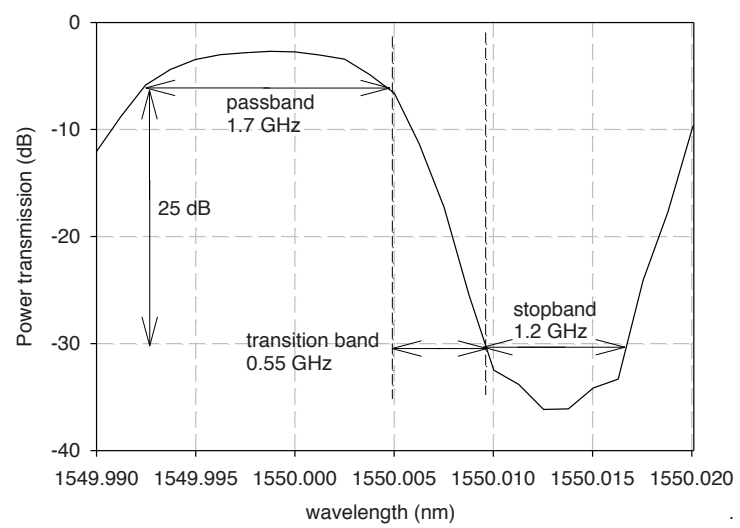

Figure 7.16: OSBF power transfer of SKY beam former chip

\section{- Optical losses in the chip}

The SKY chip is of the same quality as the FLY chip. However, the waveguide channels on the SKY chip are on average $2 \mathrm{~cm}$ longer than those on the FLY chip, thus the corresponding minimum chip insertion loss is roughly $1 \mathrm{~dB}$ higher (measured to be approximately $13 \mathrm{~dB}$ ). Furthermore, the ORR losses and power splitting losses of the SKY chip are slightly higher due to the larger maximum delay requirement and one more signal combining stage in the OBFN. However, the optical loss differences between the channels are still well below $12 \mathrm{~dB}$, and hence can be compensated by the optical combiners in the chip.

\subsection{RF-to-RF measurements on the beam former system}

After characterizing the chips, a demonstrator of a complete beam former setup was built according to the practical scheme described in Chapter 4. The FLY beam former chip was used as the core of the setup. The laser source, MZMs, and optical detector were the same as used for the chip characterization. The optical connections were made by means of PMF patch cords and PMF splitters. Since the purpose of this setup was not to measure the best achievable system performances but to prove the concepts of beam forming using ORR-based delay elements and optical singlesideband modulated signals, a single optical detector was used in the setup for simplicity, instead of a balanced detector. In this case, the signal-to-noise ratio of the system output would be lower due to the optical intensity noise from the remaining DC component, as explained in Chapter 4.

The phase shift in each optical fiber link of the optical beam former needs to be stabilized in order to enable constructive coherent optical combining in the OBFN. 
This requires both mechanical and temperature stabilization of the fiber patch cords and splitters. To achieve this, all the optical components between the laser and beam former chip were placed on top of a metal plate, which was placed inside a custommade Styrofoam box, together with the optical chip. Eventually this stabilized the optical phases within acceptable limits for a few minutes, which turned out to be sufficient to be able to perform the measurements.

In the following three subsections the desired functionality of the system is demonstrated by presenting measurements of the spectrum of the modulated optical signal, the RF-to-RF phase response from one beam former input to the output, and the RF-to-RF power response when coherently combining four beam former input signals.

\subsubsection{Sideband suppression}

Based on the optimized OSBF power response, a demonstration of sideband filtering and carrier suppression of a modulated optical signal was performed. To match the OSBF bandwidth characteristics as shown in Figure 7.13, an NA signal with a frequency sweeping from 1 to $2 \mathrm{GHz}$ was applied to one of the MZMs. The OSBF response was shifted in frequency such that one sideband of the modulated optical signal fits in the passband and other sideband in the stopband. For this demonstration the optical heterodyning technique was used to measure the optical spectrum [6]. This was performed by combining the chip output with the output signal of a tunable laser (Santec TSL-210), using a 3-dB fiber coupler. The wavelength of the Santec laser was tuned close to the wavelength of the EM 4 laser, so that a part of the spectrum of the detector output is a frequency-shifted version of the RF spectrum of the modulated optical signal, where the frequency shift corresponds to the center frequency of the tunable laser as illustrated in Figure 7.17.

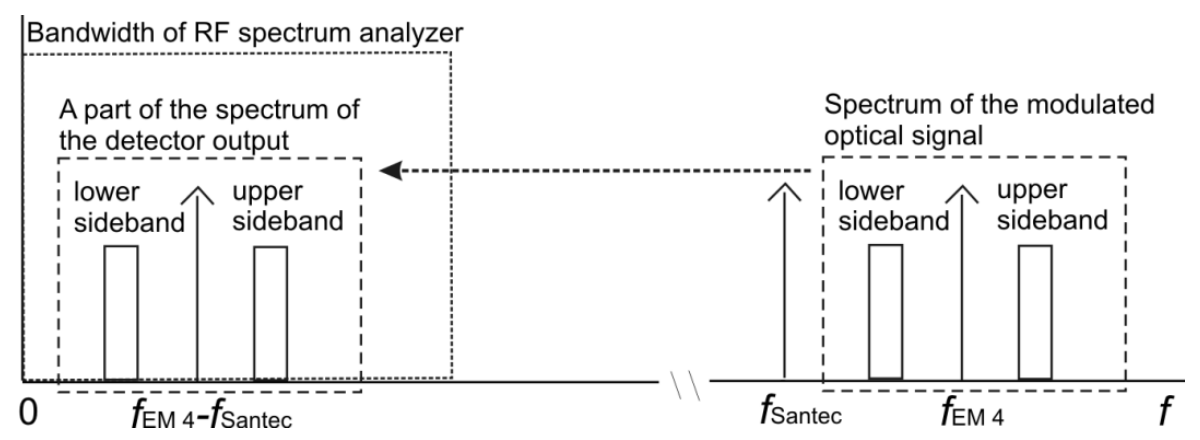

Figure 7.17: Illustration of optical heterodyning technique 
The spectrum was measured by means of an Agilent MXA N9020A signal analyzer, using the 'Maximum Hold' option in order to visualize all modulating frequencies while sweeping the modulating signal in the chosen 1 to $2 \mathrm{GHz}$ frequency range. The results are shown in Figure 7.18. The solid line corresponds to the spectrum that was obtained when the OSBF was tuned such that one sideband is suppressed, while the dashed line corresponds to the spectrum that was obtained when the OSBF was turned off (resulting in the spectrum before filtering process). The dotted line is the measured optical power transfer of the OSBF. The results clearly show that the OSBF is able to suppress one sideband of the signal by roughly $25 \mathrm{~dB}$ and leave the other sideband unchanged. The peak between two sidebands is a shifted version of the frequency line of the EM 4 laser, which was supposed to be suppressed by the MZM according to the scheme of the beam former system explained in Chapter 4. In this case the laser frequency line remains because the bias of the MZM was shifted away from the desired minimum bias point due to the bias drift of the MZM. The peak between the two sidebands is roughly at $7 \mathrm{GHz}$, which equals the frequency difference between the two lasers. Furthermore, this peak appears to have a bandwidth of about $200 \mathrm{MHz}$, which means that the center wavelength of at least one of the lasers is slightly drifting.

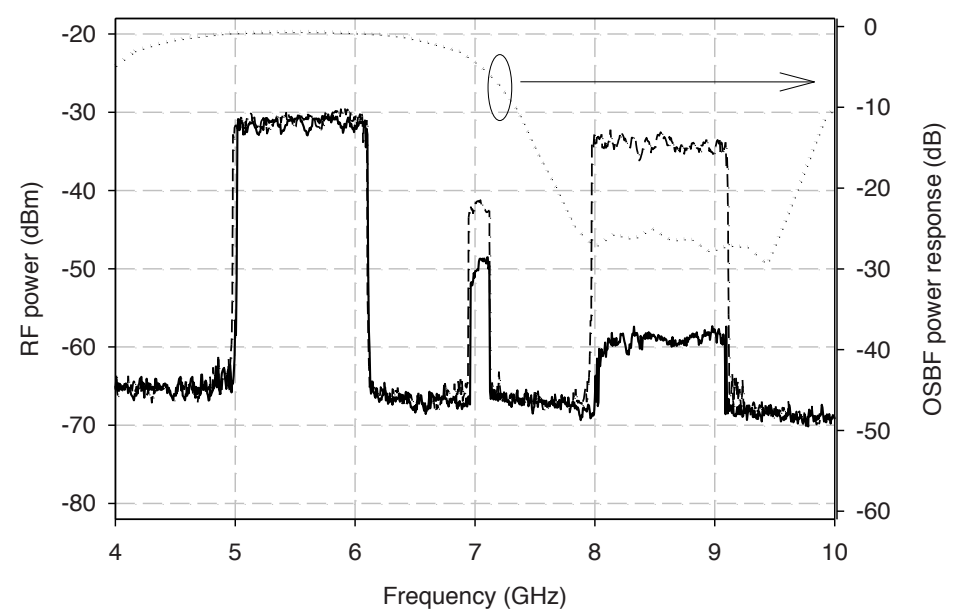

Figure 7.18: Measured spectrum of the modulated optical signal with (solid line) and without (dashed line) sideband-filtering. The dotted line is the optical power transfer of the OSBF (also shown in Figure 7.13).

\subsubsection{RF-to-RF delay generation}

The schematic of the setup for the demonstration of RF-to-RF delay generation of the beam former system is shown in Figure 7.19. 


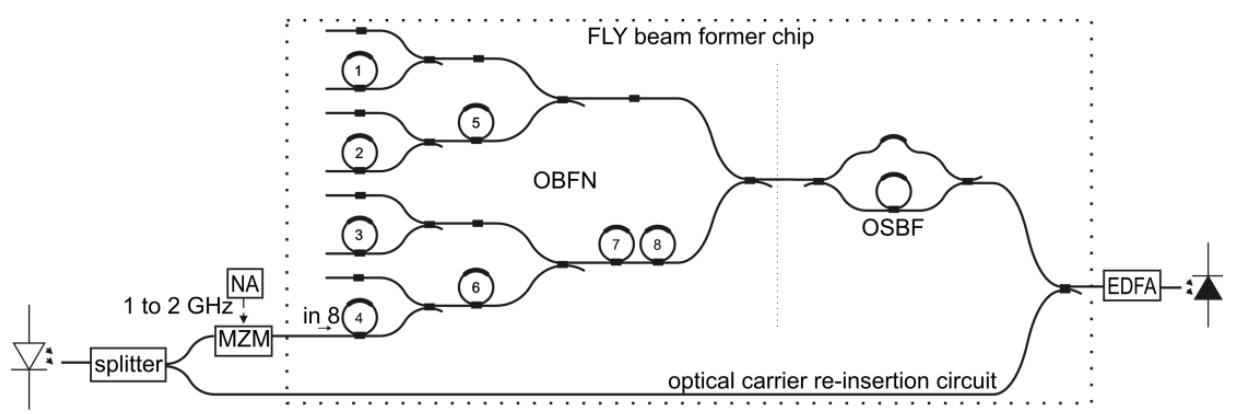

Figure 7.19: Schematic of the setup for RF-to-RF delay generation

Again, the RF signals sweeping from 1 to $2 \mathrm{GHz}$ were applied to the MZM. One fiber splitter was placed after the laser, with one of its outputs connected to the MZM. The output of the MZM and the second output of the splitter were connected to Input 8 of the OBFN (4 ORRs in the OBFN channel) and the carrier re-insertion circuit, respectively. The output of the chip was amplified by the EDFA and led to the optical detector, which was connected to the network analyzer. Three different delay settings were made on the connected OBFN channel, for $0,0.40$, and $0.63 \mathrm{~ns}$, respectively (based on the zero delay reference). The generated delay band of each delay setting was shifted to cover the lower sideband of the modulated optical signal as shown in Figure 7.20 (a). The OSBF suppressed the upper sideband, as illustrated in Figure 7.18. Signal recovery was performed after the combining between the delayed sideband and the unmodulated optical carrier (coherent optical detection). Using the network analyzer, the phase difference between the modulating signal and the recovered signal at the detector output was measured as a function of frequency, for the three different channel delay settings. Figure 7.20 (b) shows the corresponding RF-to-RF phase responses (solid lines). The dashed lines are the desired responses for the corresponding delay values. The minimum delay setting was again used as the zero reference. Besides some ripple, the obtained phase responses show a good match to the desired ones: the measured phase error is below $10 / \pi$ radians. The periodic variation of the ripples indicates that the ripples are mainly attributed to the undesired Fabry-Perot effect in the setup. The FSR of the ripples is approximately $40 \mathrm{MHz}$, which corresponds to a Fabry-Perot distance of $2.5 \mathrm{~m}$. This distance matches the length of fibers used in the setup, which means that this undesired Fabry-Perot effect is probably due to the slight reflections between the various fiber connectors in the optical signal path. However, the fiber connections can be removed when the full beam former is integrated on a single chip, including splitters and modulators. In this case, if the Fabry-Perot effect still exists, it will be not significant since the Fabry-Perot distance will be very short in the chip. 


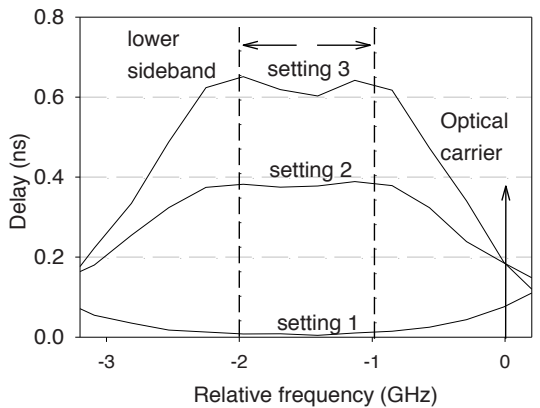

(a)

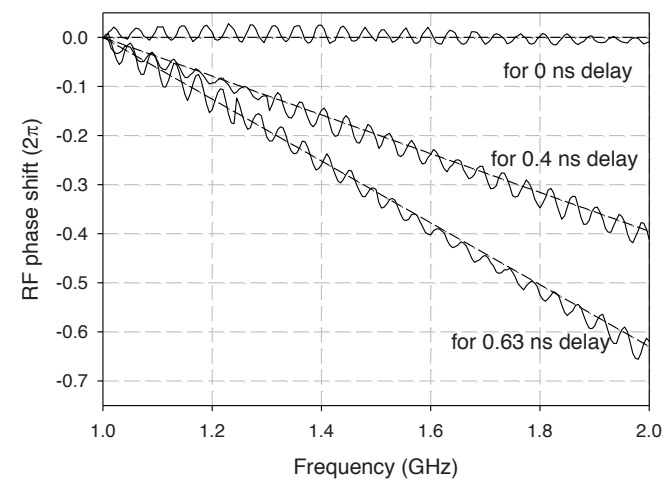

(b)

Figure 7.20: (a) The created three delay settings for input 8; (b) measured RF-toRF phase response for one path of the optical beam former, for three different delay settings (solid line). The dashed lines represent the corresponding ideal (linear) phase responses.

\subsubsection{Coherent signal combining}

The schematic of the setup for the demonstration of coherent signal combining of the beam former system is shown in Figure 7.21. For this demonstration the same NA signal frequency sweeping ( 1 to $2 \mathrm{GHz}$ ) was used. The power of the NA signal was set to $12 \mathrm{dBm}$. This NA signal was split by means of three -3-dB RF splitters, and applied to the RF inputs of four MZMs. The output of the laser was split for the optical inputs of these four MZMs and the carrier re-insertion circuit of the chip, by means of four $-3-\mathrm{dB}$ fiber splitters. The optical outputs of the MZMs were connected to the OBFN Inputs $1,2,4$, and 8 . The modulated optical signals were combined in the OBFN, filtered, combined with the unmodulated optical carrier, detected by means of the optical detector and routed back to the network analyzer. Since it was 
expected that after coherent signal combining between the OBFN channels, the power of the detected signal would be much higher than the noise level (the signal power is supposed to have a gain of $6 \mathrm{~dB}$ by each time of doubling the number of inputs), the EDFA was not added in the setup. Consequently, four paths can be identified between the output and input of the network analyzer, all of them being partly in the electrical domain and partly in the optical domain. The differences between the lengths of these paths (mainly due to differences in RF cable lengths) were removed by properly tuning the ORR-based delay elements in the OBFN.

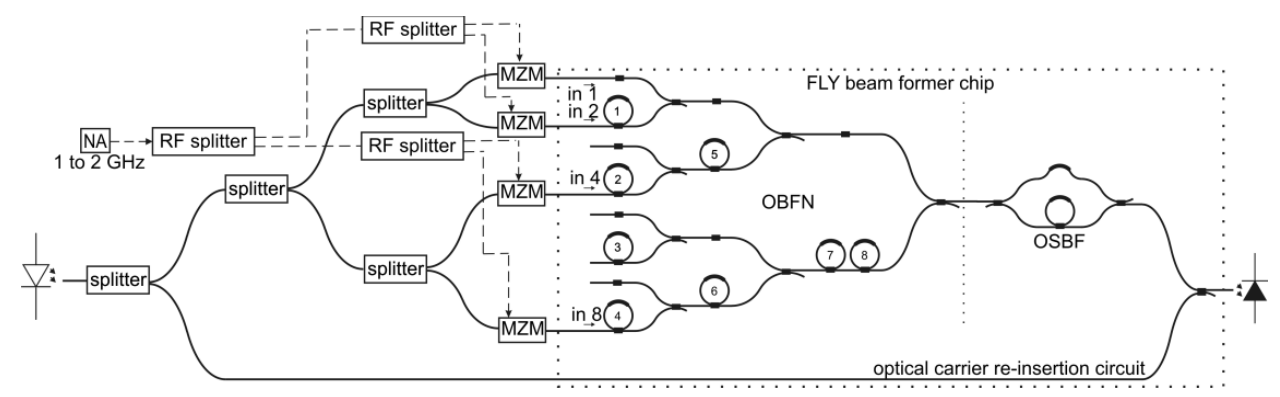

Figure 7.21: Schematic of the setup for coherent signal combining

Then, the optical phase shifters in the OBFN were tuned such that the maximum output power was achieved after the combining of the delay-synchronized modulated optical signals. The power transfer from the input of the RF splitting network to the output of the beam former was measured as a function of frequency, and is shown by the top line in Figure 7.22. Then, two outputs of the RF splitting network were disconnected from the MZMs and terminated, and the system output was measured again. In this case, the RF signal applied to the two of the inputs of the beam former maintains the same input power level. This measurement was also repeated for the other two inputs, by reconnecting the outputs of the RF splitting network and moving the terminators. The two resulting curves are the two middle ones in Figure 7.22. Finally, the RF signal was applied to the four MZM inputs individually by connecting only one of the outputs of the RF splitting network and terminating the other three. The respective measurement results are shown by the four bottom curves in Figure 7.22.

The measured curve illustrates that there was roughly $6 \mathrm{~dB}$ increase in RF power level at the output after each time of doubling the number of RF inputs and correct optical phase tuning. This demonstrates that the RF signals were coherently combined in the beam former. The ripples in the signal response were again caused by Fabry-Perot resonances, as described in the previous section. 


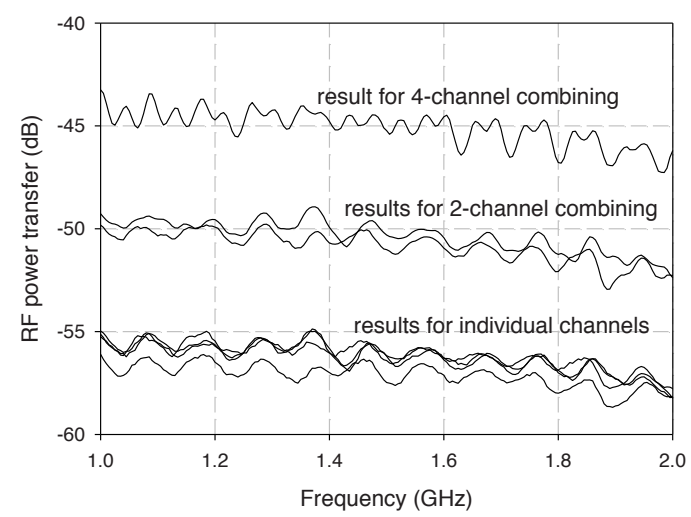

Figure 7.22: Measured RF-to-RF power response from the input of the RF splitting network to the output of the optical beamformer, when one, two, or four outputs of the RF splitting network are connected to the beam former inputs.

\subsection{Demonstration of antenna beam steering}

At the end of this research work, an experiment of antenna beam steering using the photonic beam former demonstrator was performed to demonstrate the concept of antenna control by means of an ORR-based beam former. The FLY Ku-band PAA was used in this experiment [7], which was developed by $\mathrm{NLR}^{6}$. A picture of the back side of the antenna is shown in Figure 7.23, which actually consists of $8 \times 8$ stacked Ku-band AEs.

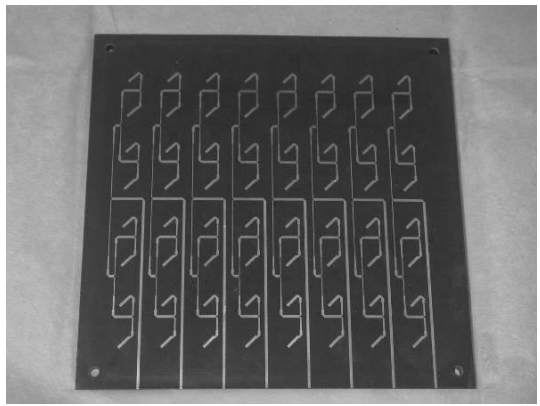

Figure 7.23: A photo of the back side of the FLY Ku-band antenna. The antenna elements in each column are combined using a combiner feed network.

\footnotetext{
${ }^{6}$ National Aerospace Laboratory (NLR). Address: Voorsterweg 31, 8316 PR Marknesse, P.O.Box 153, 8300 AD, Emmeloord, the Netherlands. Telephone: (+31) 527248444.
} 
However, in order to match the 8-input beam former system, a combiner feed network was used for this antenna. This feed network consists of 8 combiners, each of which combines a column of AEs as shown in Figure 7.23. This way, the $8 \times 8$ array antenna reduces to an 8 -element linear array antenna for beam steering in one direction, and hence can be controlled using the 8-input beam forming system (FLY photonic beam former). The measured and simulated radiation patterns of this antenna for a frequency of $11.7 \mathrm{GHz}$ are shown in Figure 7.24 (both the simulation and measurement are performed in NLR).

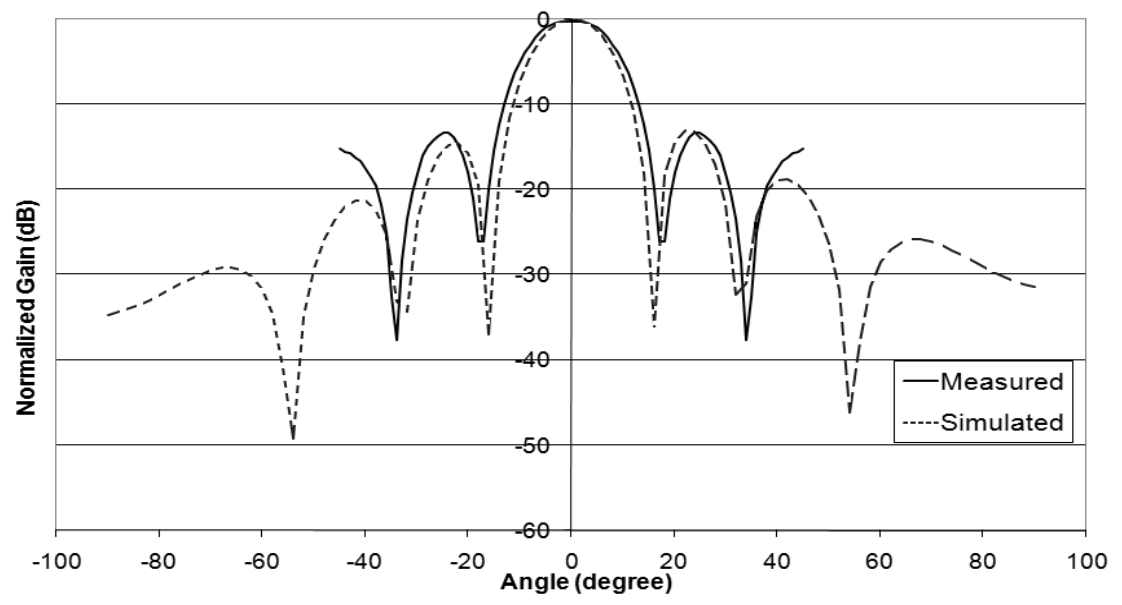

Figure 7.24: Simulated and measured antenna patterns of the 8-element FLY Ku-band antenna for frequency of $11.7 \mathrm{GHz}$, obtained by NLR.

Based on this antenna and the beam former demonstrator, the setup for the beam steering experiment was built, which is shown in Figure 7.25. Photos of this setup in the antenna test chamber of NLR are shown in Figure 7.26. For implementation simplicity, a directional radiator was used to simulate the satellite, which radiates the waves in a fixed direction. The radiator was fed with a $10.7 \mathrm{GHz} \mathrm{Ku}-$ band unmodulated carrier. The receive antenna was fixed on a rotatable base in order to achieve different relative angles of the incident waves. For frequency downconversion, eight Invacom SPV-55SM low noise blocks (LNBs) with $10 \mathrm{GHz}$ local oscillation frequency were connected to the outputs of the eight AEs to convert the received Ku-band signal into the operation frequency band of the beam former (the LNBs are phase-synchronized by means of a $10 \mathrm{MHz}$ reference signal generated by a common oscillator, and the resulting frequency after down-conversion is 700 $\mathrm{MHz}$ ). Before the MZMs, power control units were used to keep the power of modulating signals at a proper level. For the photonic beam former (FLY chip), the three OBFN delay settings presented in Section 7.2.7 were used (minimum/zero 
delay setting and the other two settings shown in Figure 7.12). However, for this experiment, the lengths of the RF paths in the setup were adjusted such that the delay setting with the $20 \mathrm{ps}$ delay step corresponded to the 0 degree beam angle (broadside), then the other two delay settings with 0 and 40 ps delay steps would steer the beam to the two opposite sides of the antenna normal by 30 degree, respectively.

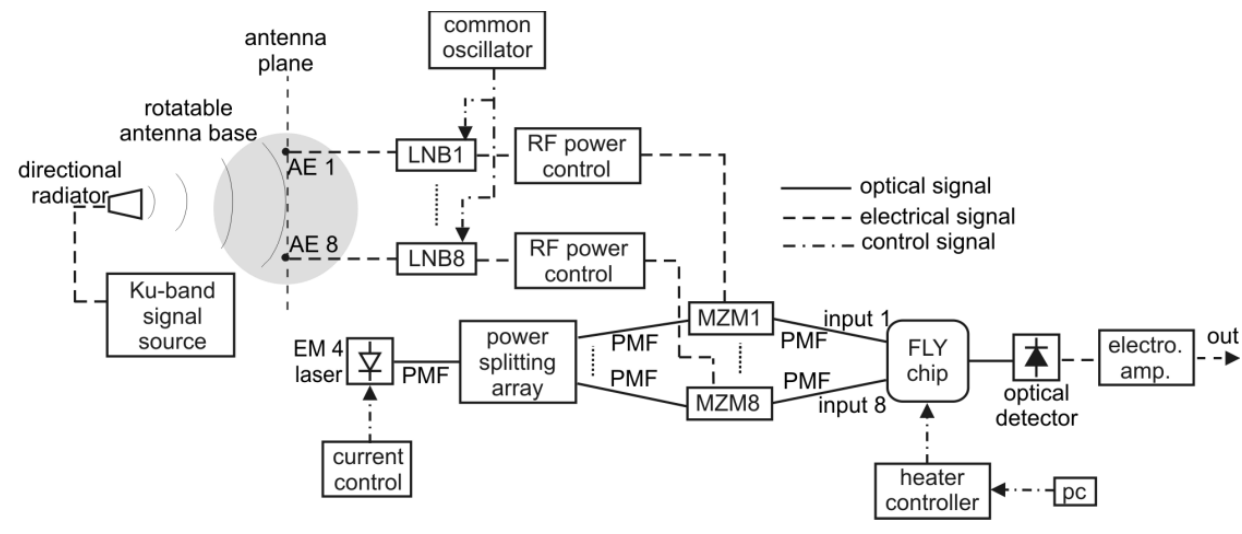

Figure 7.25: Setup for the antenna beam steering experiment

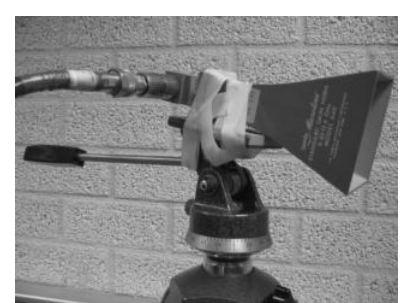

(a)

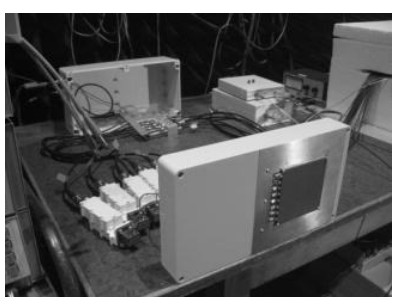

(b)

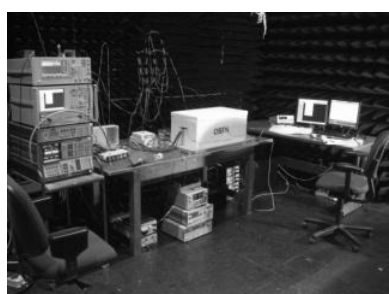

(c)

Figure 7.26: Photos of setup for the antenna beam steering experiment: (a) directional radiator; (b) FLY Ku-band PAA; (c) beam former and measurement equipments.

Due to an unexpected accident, two channels of the system became inaccessible for the measurements. Therefore, only 6 functioning channels were involved in the final experiment instead of the designed 8 channels. The simulated antenna pattern for 6 active AEs is shown in Figure 7.26. Figure 7.27 shows the spectral measurements of the system output, which were obtained when the 6-element antenna was rotated to make the incident waves change from the antenna main-beam direction to the direction of the first sidelobe. The measured signal magnitudes were approximately - 
$20 \mathrm{dBm}$ for the main beam, $-40 \mathrm{dBm}$ for the null point, and $-32 \mathrm{dBm}$ for the first sidelobe. The signal magnitude difference between the main beam and the null point is about $20 \mathrm{~dB}$, which is a bit lower than the simulated result in Figure 7.26. This is because of the slight phase deviations of the AEs in the measurement setup. For each beam angle measurement, the optical phase synchronization in the OBFN was performed manually to guarantee the maximum system output. The measured beam angles for the three OBFN delay settings are shown in Table 7.2. The results show a beam steering effect of $27^{\circ}$ when changing from the delay setting for the broadside beam angle to one of the other delay settings. This measured beam steering effect is slightly smaller than the designed 30 degree. This is due to the fact that the delay responses are not ideally flat in the delay band (as shown in Figure 7.12), so that a single frequency RF signal may experience a point in the delay band where the delay step is smaller than the average delay step of the whole delay band. For this setup, the antenna must be rotated for the 'left side' and 'right side' settings in order to point its main beam to the radiator, and in the mean time the RF cables connected to the antenna must be bended differently to allow this rotation. However, this will not be an issue for a real PAA system, since a real PAA system does not involve mechanical movements. Nevertheless, this experiment successfully demonstrated the ability of the beam former demonstrator to steer the beam of the antenna.

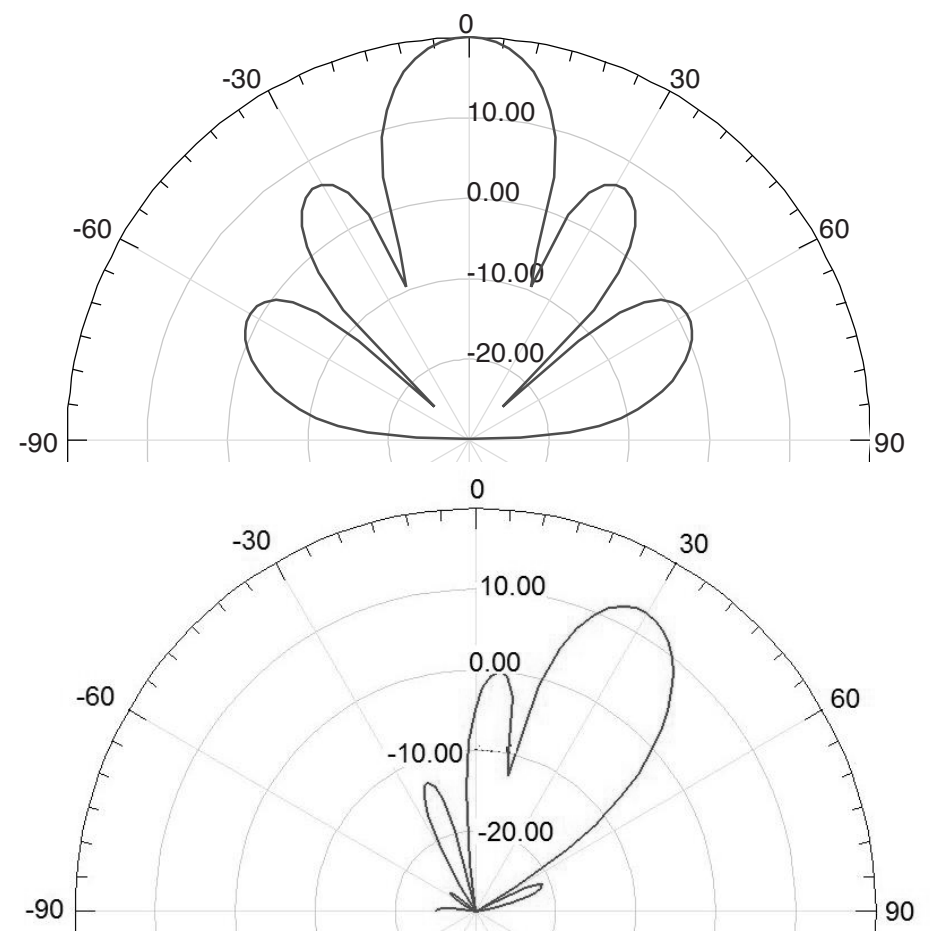

Figure 7.26: Simulated antenna pattern for 6 active AEs: (a) main beam in broadside direction; (b) main beam pointing to 30 degree. 


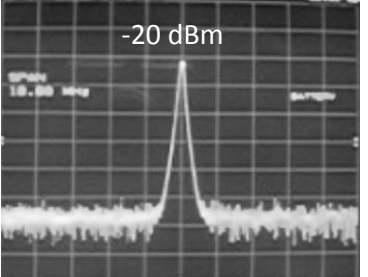

(a)

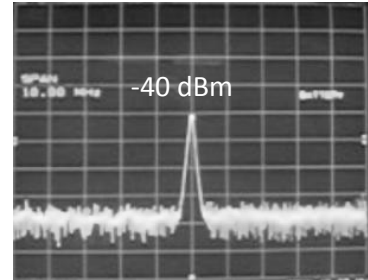

(b)

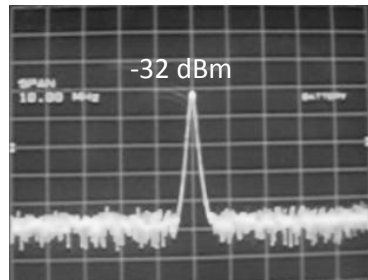

(c)

Figure 7.27: Screen shots of the system output for different incident wave directions: (a) direction of main beam; (b) direction of first null point; (c) direction of first sidelobe.

Table 7.2: Beam steering measurements for the three different OBFN delay settings shown in section 7.2.7

\begin{tabular}{|l|c|c|c|}
\hline Setting $\backslash$ pattern & Main beam & First null point & First sidelobe \\
\hline Broadside & $0^{\circ}$ & $25^{\circ} / 335^{\circ}$ & $37^{\circ} / 323^{\circ}$ \\
\hline Left side & $27^{\circ}$ & $60^{\circ} / 4^{\circ}$ & $72^{\circ} / 352^{\circ}$ \\
\hline Right side & $333^{\circ}$ & $358^{\circ} / 308^{\circ}$ & $10^{\circ} / 296^{\circ}$ \\
\hline
\end{tabular}

\subsection{Conclusions}

The components on both FLY and SKY beam former chips have been proven to function correctly. The minimum insertion losses of these two chips are approximately 12 and $13 \mathrm{~dB}$, respectively, which are still too high for real photonic beam former implementation. However, those losses have a $10 \mathrm{~dB}$ contribution from the fiber-chip coupling, which can be significantly reduced when waveguide tapering to the fiber mode profile is performed. The waveguide propagation loss is $0.6 \mathrm{~dB} / \mathrm{cm}$, which will be further reduced for the future chips since the recent improvement in the waveguide technology has reached a waveguide loss below 0.1 $\mathrm{dB} / \mathrm{cm}$. The DCs for the tunable MZI couplers of the chip have a power coupling ratio deviation of approximately 0.1 from the ideal value of 0.5 . This deviation results in slight reduction in the tuning range of the ORR couplers and the OBFN combiners. However, this is not a critical issue for system functionality, because the required tuning range of those devices can still be fulfilled. The current DC coupling ratio allows the OBFN combiners to compensate the power difference up to $12 \mathrm{~dB}$ between the two combing branches, which is more than enough for both the FLY and SKY system. The realized ORRs are able to provide continuous tunable delay. For a test channel using 7 ORRs, a delay value of $1.2 \mathrm{~ns}(36 \mathrm{~cm}$ physical distance in air) was achieved for a bandwidth of $2.5 \mathrm{GHz}$ and the maximum delay ripple of 0.1 ns. Furthermore, linearly increasing delays on different channels of a test OBFN 
were achieved, which matches the desired OBFN functionality. The power transfer of a test OSBF was also in good match with theory. Due to fabrication errors, the OSBFs on FLY and SKY chip have slight deviations in its ORR roundtrip length and the length difference between the MZI arms; however, the optimized filter responses still fulfill the corresponding system requirements. To demonstrate the beam steering ability of the FLY and SKY beam former chips, several delay settings were created on the corresponding OBFNs. For the FLY antenna, three delay settings were created which provide approximately $0 \mathrm{ps}, 20 \mathrm{ps}$ and $40 \mathrm{ps}$ delay steps between the neighboring AEs, respectively. These three delay settings can be used for beam angles of $-30,0$, and 30 degree, respectively. For the SKY 2-D array antenna, two delay settings were created which provide approximately $0 \mathrm{ps}$ and 110 ps delay steps between the neighboring AEs, respectively. These two delay settings can be used for beam angles of 0 and 10 degree, respectively, in both vertical and horizontal directions. After the device characterization, the FLY chip was used in a beam former demonstrator. Using this demonstrator, some RF-to-RF measurements were performed, which demonstrate the functionalities of the beam former chip: optical sideband suppression, RF-to-RF delay generation, and coherent signal combining. Last but not least, an experiment of antenna beam steering was performed on the FLY beam former demonstrator at NLR, and three optically controlled beam angles of $-27,0$, and 27 degree were achieved. Finally, the measurements and demonstrations presented in this chapter verify the device functionalities, and prove the concept of PAA control using ORR-based beam former system.

\section{References}

[1] W. van Etten, J. van der Plaats, Principles of Optical Fiber Communications, Prentice Hall, 1991.

[2] Mohammad Reza Salehi, Beatrice Babon, "Theoretical and Experimental Analysis of Influence of Phase-to-Intensity Noise Conversion in Interferometric Systems", J. Lightwave Technol., vol. 22, no. 6, pp. 1510-1519, June 2004.

[3] L. Zhuang, C. G. H. Roeloffzen, R. G. Heideman, A. Borreman, A. Meijerink, W. van Etten, "Single-chip optical beam forming network in LPCVD waveguide technology based on optical ring resonators," Proc. MWP'2006, Grenoble, France, 3-6 Oct. 2006, p. F1.4.

[4] F. Morichetti, A. Melloni, M. Martinelli, R. G. Heideman, A. Leinse, D. H. Geuzebroek, and A. Borreman, "Box-shaped dielectric waveguides: a new concept in integrated optics?," J. Lightwave Technol., vol. 25, no. 9, pp. 25792589, Sep. 2007.

[5] R. G. Heideman, A. Leinse, W. Hoving, R. Dekker, D. H. Geuzebroek, E. J. Klein, R. Stoffer, C. G. H. Roeloffzen, L. Zhuang, and A. Meijerink, "Large- 
scale integrated optics using TriPleXTM waveguide technology: from UV to IR," SPIE Photonics West, San Jose, CA, Jan. 24-29, 2009, paper 7221-26.

[6] Dainty C. (Ed), Laser Speckle and Related Phenomena, 1984, Sprinter Verlag, ISBN: 0387131698.

[7] H. Schippers, J. Verpoorte, P. Jorna, A. Hulzinga, A. Meijerink, C. G. H. Roeloffzen, L. Zhuang, D. A. I. Marpaung, W. van Etten, R. G. Heideman, A. Leinse, M. Wintels, "Broadband Optical beam forming for airborne phased array antenna," IEEE Aerospace conference 2009, 7-14 March 2009, pp. 1-19. 


\section{Chapter 8}

\section{Conclusions and future directions}

\subsection{Conclusions}

In this thesis a photonic beam former concept has been discussed that enables squint-free, continuous steering of broadband receive PAAs. It employs filter-based optical SSB-SC modulation, optical ring resonator (ORR)-based broadband time delay, coherent optical combining, and coherent optical detection. This photonic beam former concept provides the advantages of large instantaneous bandwidth, continuous tunability of the PAA reception angle, immunity to electromagnetic interference, reduced system complexity, enhanced signal dynamic range, and potential for integration as a compact, light-weight device.

The core of the beam former is an ORR-based optical beam forming network (OBFN) where the signals received from different antenna elements are synchronized using the delay lines formed by individual or cascade of ORRs, and subsequently combined using a signal combining circuitry. A single ORR is able to generate a delay which is even higher than its unit delay. By changing the power coupling coefficient of the ORR, the delay value can be tuned continuously. The delay band of an ORR is centered at the resonance frequency, which can be shifted by changing the additional roundtrip phase shift of the ORR. A single ORR provides limited bandwidth. The higher the peak delay at the resonance frequency is, the smaller the bandwidth will be. This is because the group delay response of an ORR is obtained by taking the negative derivative of the corresponding phase response, and therefore the area under the group delay response over one free spectral range is a constant of $2 \pi$. This delay functionality is independent of the physical dimensions of the ORR. Bandwidth enhancement can be achieved by using a cascade of multiple ORRs. By properly setting the resonance frequencies and the delay values of the individual ORRs in the cascade, a flattened delay band can be achieved with enhanced bandwidth which is roughly proportional to the number of ORRs. However, delay ripples exist in the generated delay band, which introduces distortion to the signals. To reduce the delay ripples, one can add more ORRs in the cascade or reduce somewhat bandwidth by setting the resonances frequencies of the individual ORRs closer to each other. Therefore, a cascade of multiple ORRs has tradeoffs between delay value, delay ripple, bandwidth, and the number of ORRs. In 
practice an ORR introduces loss, and the total loss of the ORR-based delay element increases with the delay value.

In the scope of this thesis, the single-wavelength OBFN scheme is used, where the optical carrier of each channel is the split of the light generated by a CW laser, and therefore coherent signal combining is needed to achieve signal intensification. This requires the coherence time of the laser to be much larger than the largest delay of the OBFN, and also requires additional optical phase shifters in the signal combining circuitry to synchronize the phases of the optical carriers. By each time combining of two channels with equal signal power, the output signal will be intensified by 6 dB. After comparing several possible OBFN architectures, it appears that the asymmetrical binary-tree structured OBFN requires the least number of ORRs resulting in the lowest system complexity, and it can be used for both linear arrays (1-dimensional) and planar (2-dimensional) PAAs.

Apart from the OBFN the $\mathrm{E} / \mathrm{O}$ and $\mathrm{O} / \mathrm{E}$ conversions are also required for the photonic beam former. A practical architecture for the entire beam former system has been found, which includes frequency down-conversion (FDC), optical sideband filter (OSBF)-based SSB-SC modulation, optical carrier reinsertion and balanced coherent optical detection. The FDC significantly relaxes the bandwidth requirements on the optical modulators; the SSB-SC modulation relaxes the bandwidth requirements on the OBFN and detectors; the use of coherent optical detection enhances the signal dynamic range of the beam former. Further, this system architecture also facilitates the system integration, since the OBFN, OSBF, and optical carrier reinsertion circuitry can be realized using a few common optical components, which can be integrated in one chip. Beam former chips including the above three modules have been realized in TriPlex ${ }^{\mathrm{TM}}$ planar waveguide technology, which is based on a CMOS-compatible LPCVD process featured by low loss and low cost. Thermo-optical tuning mechanism is used for the chip, which provides sufficient tuning speed, introduces negligible additional loss in the waveguide, and brings no difficulty in implementation. However, in this case an additional temperature control setup must be used to stabilize the chip temperature during the tuning process.

The feasibility of the proposed beam former concept has been demonstrated by means of the measurements on the fabricated beam former chips, and on the experimental beam former setup including the electro-optical and opto-electrical conversions outside the chip. The beam former chips are designed for the wavelength of $1550 \mathrm{~nm}$. The minimum insertion loss of the chips is approximately $12 \mathrm{~dB}$. The components on both FLY and SKY beam former chips prove to function correctly. The realized ORRs are able to provide continuous tunable delays. A set of 
linearly increasing delays on different channels of a test OBFN are achieved, which matches the desired OBFN functionality. For a test channel using 7 ORRs, a delay value of $1.2 \mathrm{~ns}$ ( $36 \mathrm{~cm}$ physical distance in air) is achieved for a bandwidth of 2.5 $\mathrm{GHz}$ and the maximum delay ripple of $0.1 \mathrm{~ns}$. A test OSBF was tuned to its optimized response, which is also in perfect match with theory. Three delay settings with $0 \mathrm{ps}, 20 \mathrm{ps}$ and $40 \mathrm{ps}$ delay steps between the neighboring channels are created on the FLY beam former chip, which corresponds to the theoretical beam angles of 30,0 , and 30 degree, respectively. Likewise, two delay settings with 0 ps and $110 \mathrm{ps}$ delay steps between the neighboring channels are created on the SKY beam former chip, which corresponds to the theoretical beam angles of 0 and 10 degree, respectively. Further, the FLY beam former chip was implemented in a complete beam former system (the beam former demonstrator built at $\mathrm{NLR}^{7}$ ). With this setup, some RF-to-RF measurements were performed, which demonstrate the functionalities of the beam former chip: optical sideband suppression, RF-to-RF delay generation, and coherent combining. Finally, an experiment of antenna beam steering was performed on the FLY beam former demonstrator using the three delay settings mentioned above, and three optically controlled beam angles of $-27,0$, and 27 degree are successfully obtained. The presented measurements and demonstrations verify the device functionalities, and prove the concept of PAA control using ORR-based beam former system.

To upscale the beam former, one possible way is to use a beam former chip allowing more inputs. Thanks to the binary-tree combining structure, a beam former chip with doubled number of inputs can be easily designed by simply extending the binary tree with one more stage. Another way to upscale the beam former is to use multiple beam former chips and possibly multiple lasers and detectors. In this case the entire PAA is first divided into multiple sub-PAAs, each of which is processed by an individual beam former chip. Then, the outputs of those beam former chips are further processed by another or more beam former chips until the signals received from all antenna elements are combined together. Here, a multi-wavelength OBFN scheme can be used. However, this subject is outside the focus of this thesis.

\subsection{Future directions}

\section{Photonic beam former using multiple wavelength}

This thesis focuses on the photonic beam formers using a single wavelength. In the single wavelength case, one beam direction is formed at a time. This facilitates only

\footnotetext{
${ }^{7}$ National Aerospace Laboratory (NLR). Address: Voorsterweg 31, 8316 PR Marknesse, P.O.Box 153, 8300 AD, Emmeloord, the Netherlands. Telephone: (+31) 527248444.
} 
the RF communication between two points. However, a photonic beam former has also the ability to work with multiple wavelengths simultaneously, where each wavelength can result in a different beam direction. In this case the directions of the beams are mutually related because all the wavelengths are processed using the same OBFN. Therefore the independent tuning of each wavelength is not achievable. Nevertheless, such a multi-beam system facilitates the RF systems where the communication between one and multiple points is required simultaneously. Moreover, as mentioned in Chapter 3, the photonic beam former using multiple wavelengths may reduce the OBFN complexity. Therefore, the photonic beam former using multiple wavelengths can be an interesting subject for further study in the future.

\section{Entire photonic beam former in one single chip}

It is expected that in the future an entire photonic beam former can be integrated in one single chip. So far, the photonic beam former has only the OBFN and OSBF integrated in a chip. The other optical components in the system, such as the laser, optical modulators, and optical detector, are all individually packaged devices and are interconnected using optical fibers. For those packaged optical devices packaging accounts for a large part of the device cost. Therefore, when the entire photonic beam former is integrated in one single chip, the total system cost will be significantly reduced. Moreover, the integration will not only reduce the physical dimensions of the beam former, but also increase its robustness to mechanical and thermal disturbances since the use of optical fibers is omitted. For this integration, the vertical-cavity-surface-emitting-laser and flip-chip techniques can be used for the light source, and hybrid integration techniques can be used to join different optical components together.

\section{Electrical tuning mechanism}

The current beam former chip is designed with the thermo-optical tuning mechanism. In this case the system power consumption may become an issue when the beam former system requires a large number of tuning elements (heaters). Besides, a powerful cooling platform must be used to prevent the chip temperature fluctuations. As mentioned in Chapter 5, the electro-optical tuning mechanism may also be used for the beam former chip. In this case, the optical parameters are controlled by the electrical fields supplied on the tuning elements, which do not influence the chip temperature. Electro-optical tuning mechanism also allows much higher tuning speed than the thermal tuning mechanism. However, adding electrooptical material in the beam former chip may cause higher waveguide propagation 
loss. Therefore, the feasibility of using electro-optical tuning mechanism for the beam former chip still needs to be further studied. 


\section{Appendix A}

\section{Optical parameter optimization}

As explained in Chapter 2 and 3, when the beam angle and antenna specifications are given, the desired group delay response of each OBFN channel $\hat{\tau}(\omega)$ or the corresponding phase response $\hat{\varphi}(\omega)$ are determined $(\hat{\tau}(\omega)$ is the first order derivative of $\hat{\varphi}(\omega))$. Then, the parameters of the tuning elements in the optical chip, $\phi^{\prime} \mathrm{s}$ and $\kappa^{\prime} \mathrm{s}$, should be optimized such that the real responses generated by the ORRs, $\tau(\omega)$ or $\varphi(\omega)$, approximate the desired responses. For the optimization process, first the desired and real responses are sampled at $K$ equally spaced frequency points over one FSR, then three optimization criteria can be used, namely delay criterion, phase criterion, and power criterion [1].

\section{Delay criterion}

The delay criterion is to optimize the system by means of minimizing the delay error of the system. The delay error of the system $\mu_{\mathrm{d}}$ is calculated by means of the Riemann sum of the squared delay error functions, which is given by

$$
\mu_{\mathrm{d}}=\sqrt{\sum_{k=1}^{K}[\tau(k)-\hat{\tau}(k)]^{2}}
$$

Then $\mu_{\mathrm{d}}$ can be minimized by Root Mean Square Error (RMSE) approach [2].

\section{Phase criterion}

In this case the system is optimized by minimizing the phase error of the system. The phase of the system is the integral of the corresponding delay. The phase error $\mu_{\mathrm{p}}$ is also calculated by means of the Riemann sum of the squared phase error functions, which is given by:

$$
\mu_{\mathrm{p}}=\sqrt{\sum_{k=1}^{K}[\varphi(k)-\bar{\varphi}(k)]^{2}}=\sqrt{\sum_{k=1}^{K}\left[\sum_{n=1}^{k} 2 \pi f_{S}(\tau(n)-\hat{\tau}(n))\right]^{2}}
$$

where $f_{s}$ is the sampling frequency. Then $\mu_{\mathrm{p}}$ can be minimized by RMSE approach.

\section{Power criterion}

This criterion maximizes the total output power of the system and evaluates the performance of the $M$-channel OBFN. The power error $\mu_{\text {pow }}$ is calculated by the following equation 


$$
\mu_{\text {pow }}=\sum_{k=1}^{K}\left[M-\left|\sum_{m=1}^{M} \exp \left(\mathrm{j} \varphi_{m}(k)-\mathrm{j} \widehat{\varphi_{m}}(k)\right)\right|\right]^{2}
$$

where $m$ indicates the channel number and the first $M$ in the bracket represents the desired amplitude summation of all $M$ channels. Here, the amplitude tapering is ignored. Therefore, the desired amplitude of each channel is equal and is considered to have unity value in order to simplify the equation. It is easy to recognize the part of the phase error in this formula, which is given in Eq. (A.2). But this time the complex exponentials of the phase errors of all channels are summed up, and its absolute value represents the actual amplitude summation of all the channels. Then the power error $\mu_{\text {pow }}$ can be minimized by MMSE approach.

Comparing these three criteria, the delay criterion is most straightforward and easiest for calculation. However the delay error does not play a direct role in the system output power. It is the absolute value of the sum of the channel phase vectors that determines the output power. Moreover, as far as the antenna pattern is concerned, the rise of side lobes which determines the normalized residual null level (output signal level for an undesired direction) does not directly depend on the delay error but on the phase error [3], [4]. Therefore, by using the phase criterion, the negative effect of antenna side lobes can be minimized. Even so, the phase criterion still cannot guarantee the maximum output power of the desired signal. Theoretically, the power criterion leads to the best system performance. However, the optimization process requires a non-liner programming (NLP) solving method, such as semi-quadratic programming method [5], which increases the calculation workload of the optimization process. Therefore, the selection of proper optimization criterion depends on the requirements on system performance and the calculation workload of the optimization process.

[1] R. J. Blokpoel, A. Meijerink, L. Zhuang, C. G. H. Roeloffzen, W. van Etten, "Staggered delay tuning algorithms for ring resonators in optical beam forming networks", Proceedings of the 12th Annual Symposium of the IEEE/LEOS Benelux Chapter, Brussels, Belgium, 17-18 December 2007, pp. 243-246.

[2] J. Bibby, H. Toutenburg, Prediction and Improved Estimation in Linear Models, Wiley, 1977.

[3] H. Schippers, J. Verpoorte, P. Jorna, A. Hulzinga, A. Meijerink, C. Roeloffzen, L. Zhuang, D. Marpaung, W. van Etten, R. Heideman, A. Leinse, A. Borreman, M. Hoekman, and M. Wintels, "Broadband conformal phased array with optical beam forming for airborne satellite communication," Proceeding of Aerospace Conference 2008, 2008, IEEE, pp. 1-17, March 2008.

[4] A. Meijerink, C. Roeloffzen, L. Zhuang, D. Marpaung, R. Heideman, A. Borreman, and W. van Etten, "Phased array antenna steering using a ring 
resonator-based optical beam forming network," Proceeding of the 13th IEEE/CVT Symposium Benelux, Liege, Belgium, 23 Nov. 2006.

[5] John C. G. Boot, Quadratic programming: algorithms, anomalies, applications, North-Holland Publishing, Amsterdam”, 1964. 


\section{Appendix B}

\section{Heater crosstalk compensation}

It is explained in Chapter 3 that all the tuning elements in the beam former chip are based on optical phase shifters (OPSs). Therefore, the optical parameters need to be translated to the phase shifts $\varphi$ s of the OPSs, and then the heater voltages can be calculated from $\varphi s$. In practice the actual phase shift $\varphi_{n}$ of the OPS $n$ can be expressed by

$$
\varphi_{n}=\varphi_{n_{o}}+\Delta \varphi_{n}
$$

where $\varphi_{n_{o}}$ is the initial phase shift of the OPS when no voltage is supplied on the heater, and $\Delta \varphi_{n}$ is the intentional phase shifts obtained by supplying a certain voltage $V_{n}$ on the heater. From Eq. (3.24), $\Delta \varphi_{n}$ can be given by

$$
\Delta \varphi_{n}=C \cdot P_{n}=S \cdot V_{n}^{2}
$$

where $S=C / R$ ( $R$ is the resistance of the heater and $C$ is the factor indicating the power conversion efficiency). However, when crosstalk exists between the heaters, the intentional phase shift of OPS $n, \Delta \theta_{n}$, depends not only on heater $n$ but also on other heaters. In this case $\Delta \theta_{n}$ is given by

$\Delta \varphi_{n}=S_{n, 1} \cdot V_{1}^{2}+S_{n, 2} \cdot V_{2}^{2}+\cdots+S_{n, n} \cdot V_{n}^{2}+\cdots+S_{n, N-1} \cdot V_{N-1}^{2}+S_{n, N} \cdot V_{N}^{2}$

where $N$ is the total number of heaters, $S_{i, j}$ indicates the influence of heater $j$ on the OPS $i$. Then, based on Eq. (B.3), a voltage-phase relation matrix can be obtained, which is given by

$$
\Delta \boldsymbol{\Phi}=\left[\begin{array}{c}
\Delta \varphi_{1} \\
\vdots \\
\Delta \varphi_{N}
\end{array}\right]=\left[\begin{array}{ccccc}
S_{1,1} & S_{1,2} & \ldots & S_{1, N-1} & S_{1, N} \\
S_{2,1} & S_{2,2} & \ldots & S_{2, N-1} & S_{2, N} \\
\vdots & \vdots & \ddots & \vdots & \vdots \\
S_{N-1,1} S_{N-1,2} & \ldots & S_{N-1, N-1} & S_{N-1, N} \\
S_{N, 1} & S_{N, 2} & \ldots & S_{N, N-1} & S_{N, N}
\end{array}\right] \cdot\left[\begin{array}{c}
V_{1}^{2} \\
\vdots \\
V_{N}^{2}
\end{array}\right]=\mathbf{S} \cdot \mathbf{V}^{2}
$$

Then, based on Eq. (B.4), the heater voltages with the crosstalk compensation can be calculated by

$$
\mathbf{V}^{2}=\mathbf{S}^{-1} \cdot \Delta \boldsymbol{\Phi}=\mathbf{S}^{-1} \cdot\left(\boldsymbol{\Phi}-\boldsymbol{\Phi}_{\mathbf{o}}\right)
$$

Here, $\mathbf{S}$ and $\boldsymbol{\Phi}_{\mathbf{0}}$ are the characteristics of the beam former chip, and can be obtained by means of measurement. 


\section{Publications}

\section{Journal Papers}

W. C. L. Hopman, H. J. W. M. Hoekstra, R. Dekker, L. Zhuang, R. M. de Ridder, "Far-field scattering microscopy applied to analysis of slow light, power enhancement, and delay times in uniform Bragg waveguide gratings," Optics Express (ISSN: 1094-4087), vol. 15, no. 4, pp. 1851-1870, February 2007.

L. Zhuang, C. G. H. Roeloffzen, R. G. Heideman, A. Borreman, A. Meijerink, W. van Etten, "Single-chip ring resonator-based $1 \times 8$ optical beam forming network in CMOS-compatible waveguide technology," IEEE Photonics Technology Letters, vol. 19, no. 15, pp. 1130-1132, August 2007.

H. P. Uranus, L. Zhuang, C. G. H. Roeloffzen, H. J. W. M. Hoekstra, "Pulse advancement and delay in an integrated-optical two-port ring-resonator circuit: direct experimental observations," Optics Letters, vol. 32, Issue 17, pp. 2620-2622, August 2007.

A. Meijerink, C.G.H. Roelozffzen, R. Meijerink, L. Zhuang, D. A. I. Marpaung, M.J. Bentum, M. Burla, J. Verpoorte, P. Jorna, A. Hulzinga, and W. van Etten, "Novel Ring Resonator-Based Integrated Photonic Beamformer for Broadband Phased Array Receive Antennas-Part I: Design and Performance analysis," IEEE Journal of Lightwave Technology, vol.28, no. 1, pp. 3-18, January 2010.

L. Zhuang, C.G.H. Roelozffzen, A. Meijerink, M. Burla, D. A. I. Marpaung, A. Leinse, M. Hoekman, R. Heideman, and W. van Etten, "Novel Ring ResonatorBased Integrated Photonic Beamformer for Broadband Phased Array Receive Antennas-Part II: Experimental prototype analysis," IEEE Journal of Lightwave Technology, vol 28, no. 1, pp. 19-31, January 2010.

\section{Conference Papers}

L. Zhuang, C. G. H. Roeloffzen, W. C. van Etten, "Continuously tunable optical delay line," Proceedings of the 12th Symposium on Communications and Vehicular Technology in the Benelux (CDROM, ISBN: 90-365-2264-1), Enschede, The Netherlands, 3rd November 2005, p. P9.

C. G. H. Roeloffzen, L. Zhuang, R. G. Heideman, A. Borreman, W. C. van Etten, "Ring resonator-based tunable optical delay line in LPCVD waveguide technology," Proceedings of the Ninth Annual Symposium of the IEEE/LEOS Benelux Chapter, Mons, Belgium, 1-2 December 2005, pp. 79-82. 
L. Zhuang, Chris G.H. Roeloffzen, Wim C. van Etten, "Ring resonator-based continuously tunable optical delay line," URSI Benelux Meeting, Eindhoven, The Netherlands, 12th May 2006, p. P23.

L. Zhuang, C. G. H. Roeloffzen, R. G. Heideman, A. Borreman, A. Meijerink, W. van Etten, "Single-chip optical beam forming network in LPCVD waveguide technology based on optical ring resonators," Proceedings of the International Topical Meeting on Microwave Photonics (MWP'2006), Grenoble, France, 3-6 October 2006, p. F1.4.

A. Meijerink, C. G. H. Roeloffzen, L. Zhuang, D. A. I. Marpaung, R. G. Heideman, A. Borreman, W. van Etten, "Phased array antenna steering using a ring resonatorbased optical beam forming network," Proceedings of the 13th Symposium on Communications and Vehicular Technology in the Benelux, Liège, Belgium, 23 November 2006, pp. 7-12.

L. Zhuang, C. G. H. Roeloffzen, R. G. Heideman, A. Borreman, A. Meijerink, W. van Etten, "Ring resonator-based single-chip 1x8 optical beam forming network in LPCVD waveguide technology," Proceedings of the Eleventh Annual Symposium of the IEEE/LEOS Benelux Chapter, Eindhoven, The Netherlands, 30 November-1 December 2006, pp. 45-48.

R. G. Heideman, D. H. Geuzebroek, A. Leinse, A. Melloni, F. Morichetti, C. G. H. Roeloffzen, A. Meijerink, L. Zhuang, W. van Etten, E. Klein, A. Driessen, "Low loss, high contrast optical waveguides based on CMOS compatible LPCVD processing" (invited), Proceedings of the 13th European Conference on Integrated Optics (ECIO'2007), Copenhagen, Denmark, 25-27 April 2007, p. WB0.

H. P. Uranus, L. Zhuang, C. G. H. Roeloffzen, H. J. W. M. Hoekstra, "Direct Experimental Observation of Pulse Temporal Behavior in Integrated-Optical RingResonator with Negative Group Velocity," Proceedings of the 13th European Conference on Integrated Optics (ECIO'2007), Copenhagen, Denmark, 25-27 April 2007, post-deadline paper.

L. Zhuang, A. Meijerink, C. G. H. Roeloffzen, D. A. I. Marpaung, J. Peña Hevilla, W. van Etten, R. G. Heideman, A. Leinse, M. Hoekman, "Phased array receive antenna steering system using a ring resonator-based optical beam forming network and filter-based optical SSB-SC modulation," Proceedings of the International Topical Meeting on Microwave Photonics (MWP'2007), Victoria, BC Canada, 3-5 October 2007, p. Th-3.3, pp. 88-91.

L. Zhuang, A. Meijerink, C. G. H. Roeloffzen, D. A. I. Marpaung, R. G. Heideman, M. Hoekman, A. Borreman, D. H. Geuzebroek, A. Leinse, W. van Etten, "Experimental prototype of a novel ring resonator-based optical beamformer 
system," Proceedings of the LEOS Annual Meeting 2007, Lake Buena Vista, Florida, 21-25 October 2007, p. MM3, pp. 112-113.

L. Zhuang, A. Meijerink, C. G. H. Roeloffzen, D. A. I. Marpaung, R. G. Heideman, M. Hoekman, A. Borreman, A. Leinse, W. van Etten, "Novel ring resonator-based optical beamformer system and experimental results," Proceedings of the 12th Annual Symposium of the IEEE/LEOS Benelux Chapter, Brussels, Belgium, 17-18 December 2007, pp. 239-242.

R. J. Blokpoel, A. Meijerink, L. Zhuang, C. G. H. Roeloffzen, W. van Etten, "Staggered delay tuning algorithms for ring resonators in optical beam forming networks," Proceedings of the 12th Annual Symposium of the IEEE/LEOS Benelux Chapter, Brussels, Belgium, 17-18 December 2007, pp. 243-246.

H. Schippers, J. Verpoorte, P. Jorna, A. Hulzinga, A. Meijerink, C. Roeloffzen, L. Zhuang, D. Marpaung, W. van Etten, R. G. Heideman, A. Leinse, A. Borreman, M. Hoekman, M. Wintels, "Broadband Conformal Phased Array with Optical Beam forming for Airborne Satellite Communication," Proceedings of the IEEE Aerospace Conference 2008, Big Sky, Montana, 1-8 March 2008, p. 3.0102.

J. W. van 't Klooster, C. Roeloffzen, A. Meijerink, L. Zhuang, D. Marpaung, W. van Etten, R. G. Heideman, A. Leinse, H. Schippers, J. Verpoorte, M. Wintels, "Design of a Ring Resonator-Based Optical Beam Forming Network for Phased Array Receive Antennas," 30th ESA Antenna Workshop on Antennas for Earth Observation, Science, Telecommunication and Navigation Space Missions, ESA/ESTEC, Noordwijk, The Netherlands, 27-30 May, p. 10.6, pp. 403-406.

H. Schippers, J. Verpoorte, P. Jorna, A. Hulzinga, A. Meijerink, C. G. H. Roeloffzen, L. Zhuang, D. A. I. Marpaung, W. van Etten, R. G. Heideman, A. Leinse, A. Borreman, M. Hoekman, M. Wintels, "Dual-Frequency Phased Array Antennas with Optical Beam forming for Airborne Satellite Communication," 30th ESA Antenna Workshop on Antennas for Earth Observation, Science, Telecommunication and Navigation Space Missions, ESA/ESTEC, Noordwijk, The Netherlands, 27-30 May, p. 10.10, pp. 419-422.

C. G. H. Roeloffzen, A. Meijerink, L. Zhuang, D. A. I. Marpaung, W. van Etten, R. G. Heideman, A. Leinse, M. Hoekman, "Integrated photonic beamformer employing continuously tunable ring resonator-based delays in CMOS-compatible LPCVD waveguide technology" (invited), Asia-Pacific Optical Communications (APOC) 2008, Hangzhou, China, 26-30 October 2008, p. 7135-59.

L. Zhuang, A. Meijerink, C. G. H. Roeloffzen, D. A. I. Marpaung, R. G. Heideman, M. Hoekman, A. Leinse, W. van Etten, "Novel ring resonator-based optical beamformer for broadband phased array receive antennas," Proceedings of the 
LEOS Annual Meeting 2008, Newport Beach, California, 9-13 November 2008, p. MB3, pp. 20-21.

L. Zhuang, A. Meijerink, C. G. H. Roeloffzen, D. A. I. Marpaung, R. G. Heideman, M. Hoekman, A. Leinse, W. van Etten, "Broadband phased array antenna steering by means of coherent signal combining in an integrated ring resonator-based optical beamformer," Proceedings of the 13th Annual Symposium of the IEEE/LEOS Benelux Chapter, Enschede, The Netherlands, 27-28 November 2008, pp. 71-74.

René Heideman, Arne Leinse, Willem Hoving, Ronald Dekker, Douwe Geuzebroek, Edwin Klein, Remco Stoffer, Chris Roeloffzen, Leimeng Zhuang, Arjan Meijerink, "Large-scale integrated optics using TriPleX ${ }^{\mathrm{TM}}$ waveguide technology: from UV to IR" (Invited Paper), SPIE Photonics West, San Jose, California, 24-29 January 2009, p. $7221-26$.

H. Schippers, J. Verpoorte, P. Jorna, A. Hulzinga, L. Zhuang, A. Meijerink, C. Roeloffzen, D. Marpaung, W. van Etten, R. G. Heideman, A. Leinse, M. Wintels, "Broadband optical beam forming for airborne phased array antenna," Proceedings of the IEEE Aerospace Conference 2009, Big Sky, Montana, 7-14 March 2009, p. 1507 , to be published.

\section{Popular scientific publications}

Arjan Meijerink, Chris Roeloffzen, Leimeng Zhuang, David Marpaung, Wim van Etten, "Optical beam forming for phased array antennas," De Vonk (Monthly magazine of E.T.S.V. Scintilla), vol. 25, no. 1, Oct. 2006, pp. 4-7.

A. Meijerink, C. G. H. Roeloffzen, L. Zhuang, D. A. I. Marpaung, R. G. Heideman, A. Borreman, W. van Etten, "Optical beam forming for phased array antennas," Fotonica Magazine, vol. 31, no. 4, pp. 5-9, February 2007.

L. Zhuang, C.G.H. Roeloffzen, R.G. Heideman, A. Borreman, A. Meijerink, W. van Etten, "Single-chip ring resonator-based $1 \times 8$ optical beam forming network in CMOS-compatible waveguide technology," NIRICT Kick-off congress, Utrecht, The Netherlands, 22 March 2007.

A. Meijerink, C. G. H. Roeloffzen, L. Zhuang, D. A. I. Marpaung, R. G. Heideman, A. Borreman, W. van Etten, "Optische bundelvormer voor breedbandige phased array antennes," Fotonica Evenement 2007, The Hague, The Netherlands, 3 April 2007.

Arjan Meijerink, Chris Roeloffzen, David Marpaung, Leimeng Zhuang, Wim van Etten, Arne Leinse, Marcel Hoekman, René Heideman, "RF Photonics technology for phased array antenna applications," Fotonica Evenement 2008, Nieuwegein, The Netherlands, 2 April 2008. 


\section{Acknowledgement}

After a very long, more than one and half year, and painful waiting, finally I am reaching the point to put an end to the writing of this thesis.

Being a $\mathrm{PhD}$ student is enjoyable but writing the thesis is really killing as everyone says. This is a truth. Now I have experienced it and proven it. In my case this past one and half year is filled with pain, tiredness, stress, and sometimes sleeplessness. I would say it is the hardest time I have ever had, and it seemed much longer than it was. During this period, many changes happened to me and to the things around me, which made the writing from hard to even harder. But, on the other hand, it has been a good lesson about life, which forced me to think more, so that I got better understanding of myself, my career, and many other important points of my life. However hard it was, I could still get a good feeling when I saw the word by word progress of the thesis, even when I could just add two lines after a whole day of scratching the head. And just this feeling encouraged me and kept me going on. On the other hand, frustration came also from time to time, and at a certain point I doubted the meaning of taking so much bother to write a book of all those things which have been already well studied and concluded. Is it just a waste of time? Definitely not! Because later I found out that those knowledge points are just like the cornflakes of a puzzle. If you want to see the whole picture, you need to join them all together in one frame.

For those $\mathrm{PhD}$ students who are still struggling with their thesis, "Please hang on there, don't worry, you are doing something certainly worthwhile, and you will get it sooner or later. Believe me, it is just a matter of perseverance." Lucky me, the finishing line is now lying right in front of me. I am really proud to have this book in my hand, which is undoubtedly the greatest achievement I have ever had. Nevertheless, this achievement is not only a work of me. It is also thanks to those who have provided contributions to make it completed. Hereby, I would like to express my sincere gratitude to the people who guided me, helped me, inspired me, and cheered me up in my $\mathrm{PhD}$ study time.

In the first place I would like to thank my supervisor Prof. Wim van Etten and my daily advisor Dr. Chris Roeloffzen for their clear guidance, patient explanations, and frequent encouragement as well as inspiration during my $\mathrm{PhD}$ study. I am also grateful to them for sacrificing their precious time in reviewing and correcting my thesis.

I would like to thank University of Twente and Agentschap NL (SenterNovem) for providing the great studying and working environment and financially sponsoring the BPB project and SMART project, respectively. 
I am thankful to all the committee members for spending time in reading my thesis and for their valuable comments.

I would like to express my thanks to my colleges at LioniX, NLR and Astron, namely Marcel Hoekman, Arne Leinse, Adriaan Hulzinga, and Klaas Dijkstra for their active cooperation in the project and their contributions to my thesis.

I would like to say "Thank You" to my fellow TE folks (both former and current), Alejandro, Alex, Annemiek, Arjan, Anne, Beneoumeur, David, Edward, Elangovan, Frank, Frits, Jack, Jan-Willem, Joe, Le, Liang, Lilian, Mark, Maurizio, Olga, Rajan, Rajeev, Reza, Roelof, Willem, Yakup, and also the other TEers. I thank them for being not only my colleges but also my close friends. They give me support, help, fun and happiness. When they are around me, I feel warm, relaxed, and at home.

Also, all the friends I have met in The Netherlands deserve a big thank word from me for keeping me not alone and cheering me up all the time. From them I have learned more about friendship, sincerity, and love.

Last but not least, I owe all I have achieved today to my beloved family, especially my parents and my older brother. Without them I could not have achieved so much. In my easy and hard, they are always with me, being the shield and being the support. Here, I would also like to express an extra gratitude to my mom, an openminded Chinese woman. It was her who brought me on the way to explore this world. And it was her encouragement and inspiration that has made me who I am.

Leimeng Zhuang 
The publishing process of this thesis is accomplished with the supports from

University of Twente

Enschede - The Netherlands www.utwente.nl

\author{
Vadeno \\ www.vadeno.nl
}




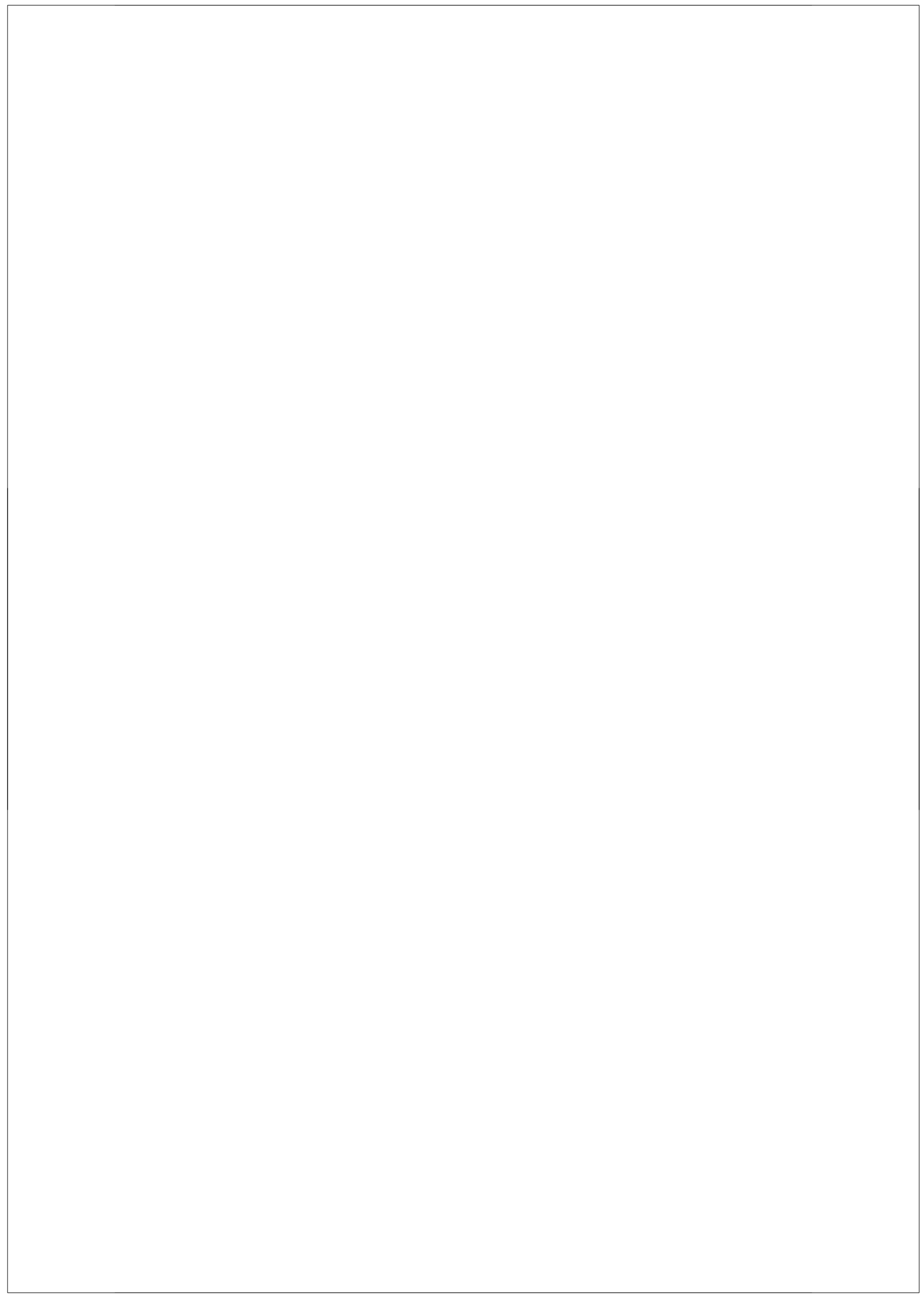

